\title{
Characterization of diffractive optical lenses for use in MEMS integrated optical monitoring
}

Christopher W. Hollandsworth

West Virginia University

Follow this and additional works at: https://researchrepository.wvu.edu/etd

\section{Recommended Citation}

Hollandsworth, Christopher W., "Characterization of diffractive optical lenses for use in MEMS integrated optical monitoring" (2006). Graduate Theses, Dissertations, and Problem Reports. 1705.

https://researchrepository.wvu.edu/etd/1705

This Thesis is protected by copyright and/or related rights. It has been brought to you by the The Research Repository @ WVU with permission from the rights-holder(s). You are free to use this Thesis in any way that is permitted by the copyright and related rights legislation that applies to your use. For other uses you must obtain permission from the rights-holder(s) directly, unless additional rights are indicated by a Creative Commons license in the record and/ or on the work itself. This Thesis has been accepted for inclusion in WVU Graduate Theses, Dissertations, and Problem Reports collection by an authorized administrator of The Research Repository @ WVU. For more information, please contact researchrepository@mail.wvu.edu. 


\title{
CHARACTERIZATION OF DIFFRACTIVE OPTICAL LENSES FOR USE IN MEMS INTEGRATED OPTICAL MONITORING
}

\author{
Christopher W. Hollandsworth \\ Thesis submitted to the \\ College of Engineering and Mineral Resources at \\ West Virginia University \\ in partial fulfillment of the requirements \\ for the degree of
}

MASTER OF SCIENCE

in

\section{ELECTRICAL ENGINEERING}

Dr. Lawrence Hornak, Ph.D., Chair

Dr.Parviz Famouri, Ph.D.

Dr. Dimitris Korakakis, Ph.D.

Lane Department of Computer Science and Electrical Engineering

West Virginia University

Morgantown, West Virginia

2006

Keywords: MEMS, Optical Monitoring, DOE, Fresnel Lens 


\title{
ABSTRACT \\ THEORY AND CHARACTEREIZATION OF DIFFRACTIVE OPTICAL LENSES FOR USE IN MEMS INTEGRATED OPTICAL MONITORING
}

\author{
CHRISTOPHER W. HOLLANDSWORTH
}

Micro Electro Mechanical Machines (MEMS) are finding use in an increasing number of applications. Many of these applications, especially safety critical ones, require continuous knowledge of the operational status of the MEMS device over its lifetime. In-situ monitoring of the operational status of these devices is better suited to optical methods which provide a non-invasive way of continuously determining device position as a function of time. This data is central to determining the MEMS state and operational condition. Whether the optical source is internal to the MEMS package or external to the package and introduced via optical fiber, lenses will be required within the package to focus the MEMS optical probe beam to and from the test device. The need for hybrid co-integration with the MEMS die within its package results in physical dimension and focal length constraints on the lenses used and points toward the applicability of diffractive optical lenses to realize the goal of MEMS insitu integrated optical monitoring. The spot diameter must be of a width comparable to any lateral displacement that must be measured so that acceptable resolution of the motion can be observed. Diffractive lenses are capable of meeting all of these requirements. Previous work designed and fabricated Fresnel binary phase diffractive lenses in borofloat glass substrates as a means to achieve the required lens function for either a visible (632 $\mathrm{nm}$ wavelength) or near IR $(1310 \mathrm{~nm})$ probe beam. This work undertakes the full optical characterization of these previously fabricated lenses, compares their performance (spot size, focal length, efficiency) to that expected from theory, and determines if they meet the original lens design criteria, These results enable an initial assessment of these lenses for use in MEMS in-situ lifetime optical monitoring. 


\section{ACKNOWLEDGMENTS}

I would like to thank my committee chair Dr. Larry Hornak for his invaluable help and guidance, without which this may not have been possible. I want to thank my committee members Dr. Dimitris Korakakis and Dr. Parviz Famouri. I also want to thank Dr. Kolin Brown for his assistance in the laboratory. My thanks go to the following MEMS group members for their assistance through my work: Afshin Izadian, Deepasree Konduparthi, John Harmon, William McCormick, and Divya Pisharoty.

I want to thank my family for their support through this time, especially my fiancé Kelly Showalter for her love, encouragement, and patience. 


\section{Contents}

$\begin{array}{lll}\text { Abstract } & \text { ii }\end{array}$

Acknowledgements

Contents

List of Figures $\quad$ vi

$\begin{array}{lll}\text { List of Tables } & \text { xi }\end{array}$

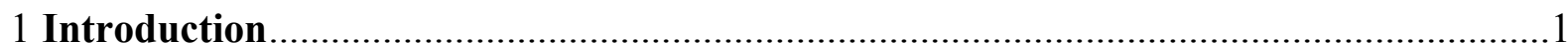

1.1 MEMS and Device Monitoring Needs ........................................................ 1

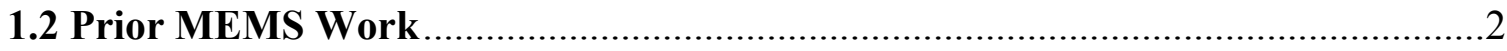

1.3 Previous Integrated Optical Monitoring Work .................................................

1.4 Direction and Organization of Thesis ......................................................

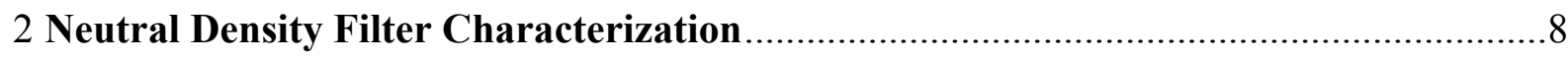

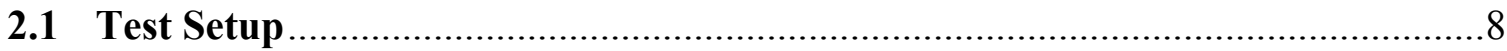

2.2 Measured Neutral Density Filter Transmission Percentages...........................

2.4 Transmission Through Neutral Density Filters for Non-Normal Incidence .....17

2.5 Point Distortions from Particulate Matter on Filters .................................... 19

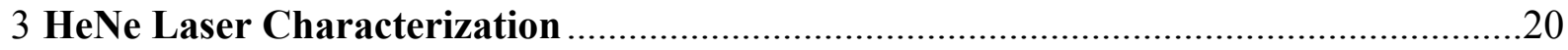

3.1 Gaussian Beam Theory and Gas Lasers ................................................ 20

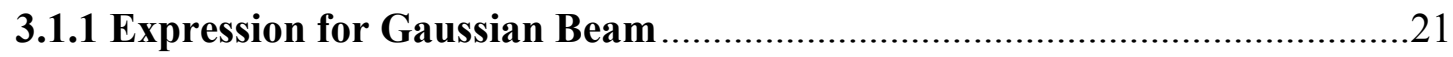

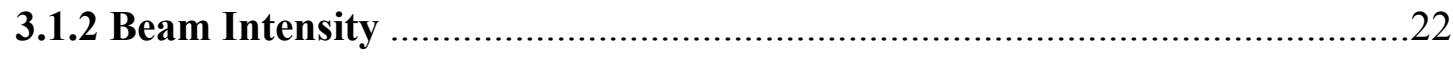

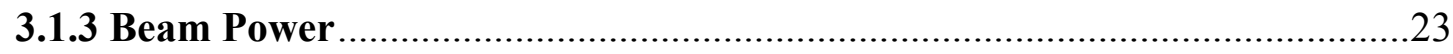

3.1.4 Beam Radius, Divergence, and Depth of Focus .................................23

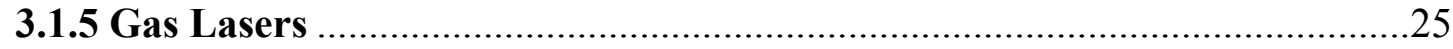




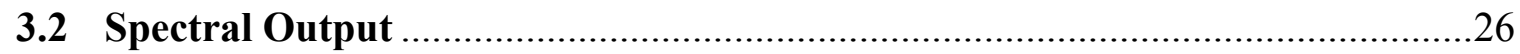

3.3 Waist Location, Waist Size, and Angular Divergence ………………………....28

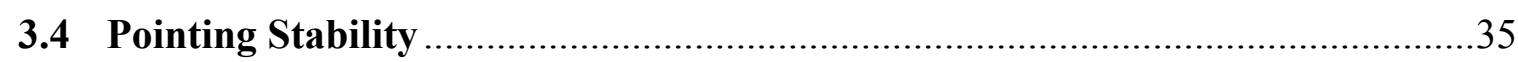

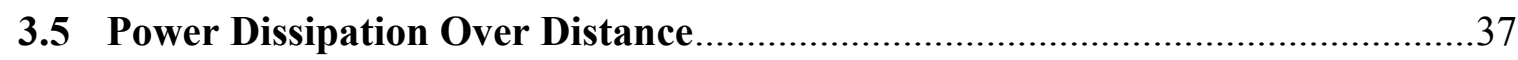

3.6 In-Plane Power Distribution and Beam Shape …………………......................

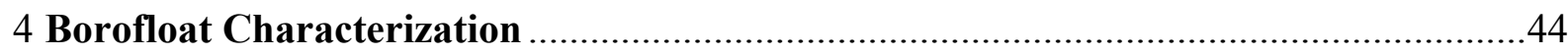

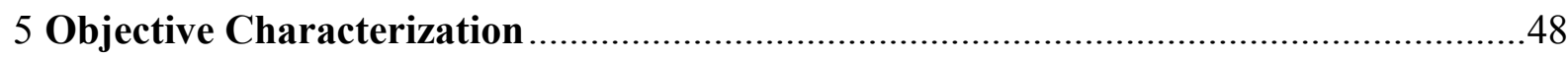

6 Electromagnetic Propagation and Optical Lens Theory ..............................................52

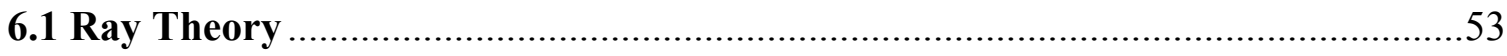

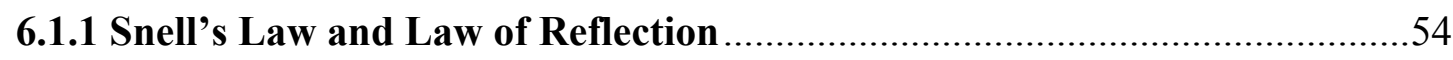

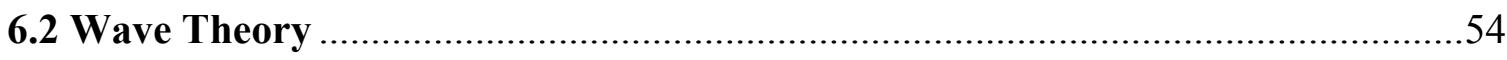

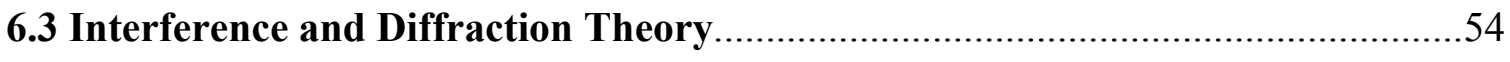

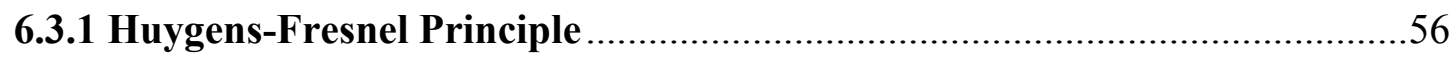

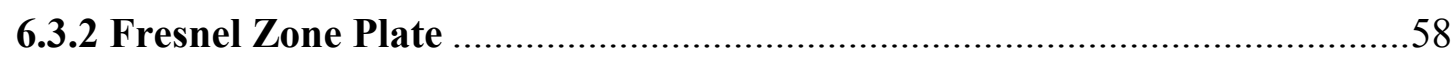

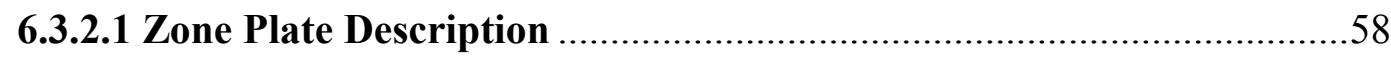

6.3.2.2 Material Selection and Fabrication Techniques ..................................59

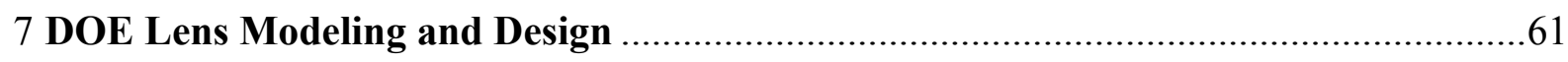

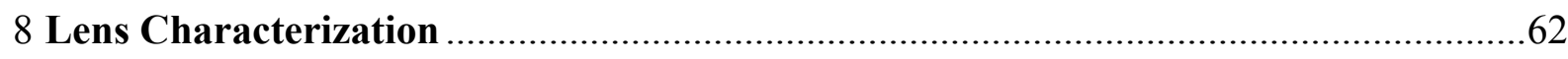

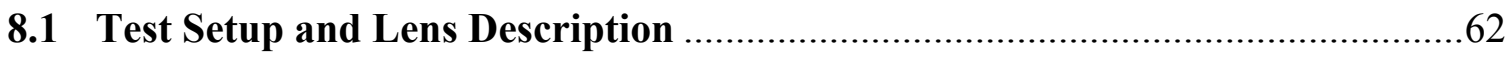

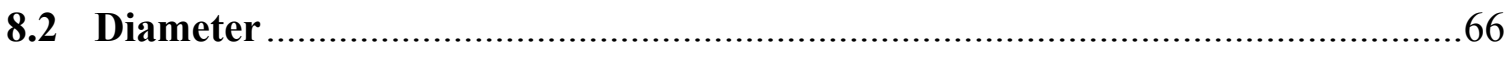

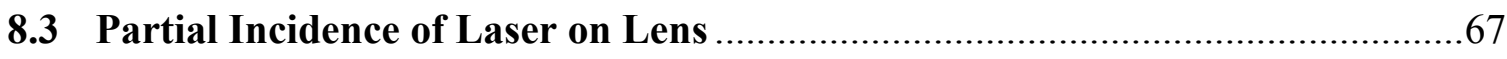

8.4 Focal Point and Focal Range …………………….........................................

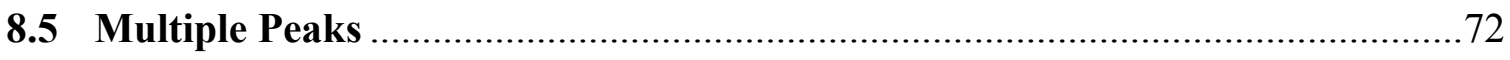

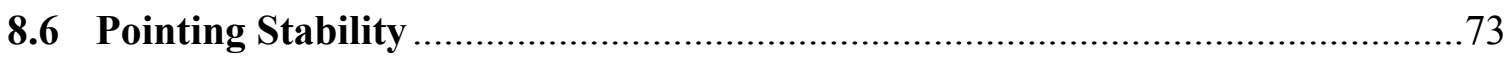

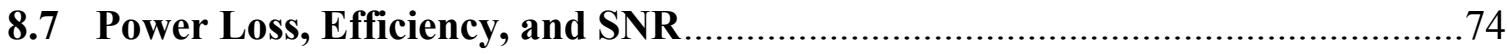


8.8 In-Plane Power Distribution and Beam Shape .......................................... 77

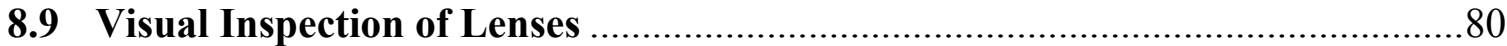

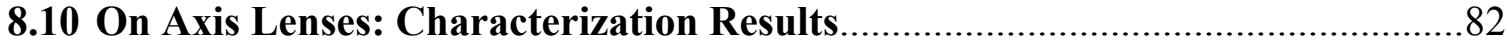

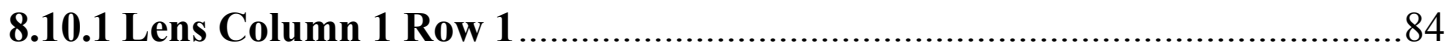

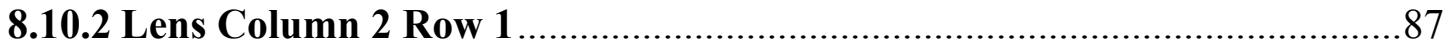

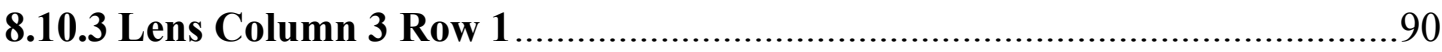

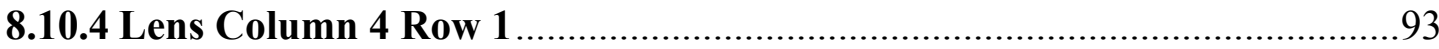

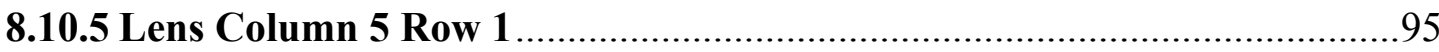

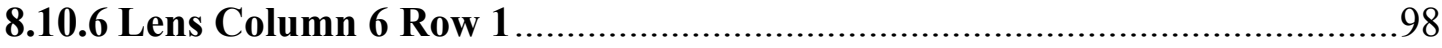

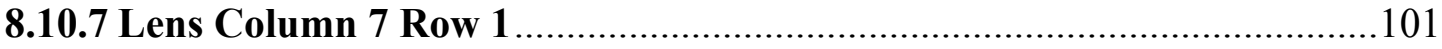

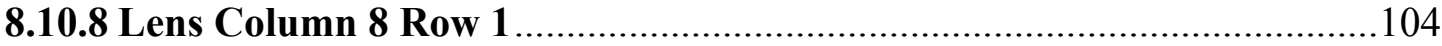

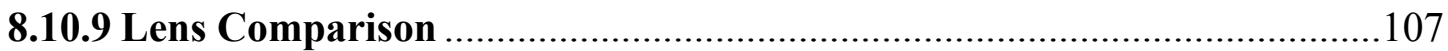

8.11 Discussion of Lens Results and Connection to Diffraction Theory .............108

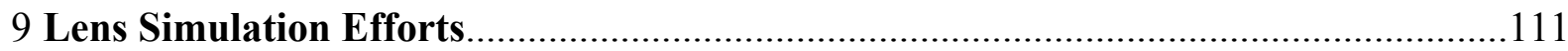

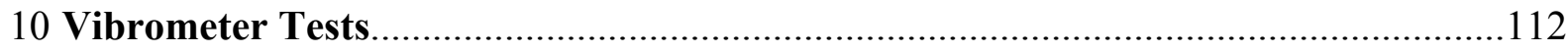

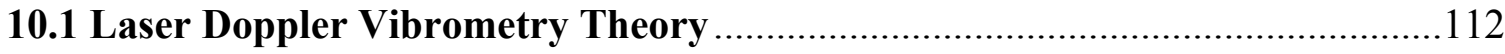

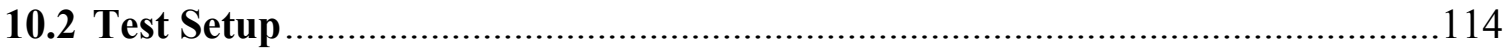

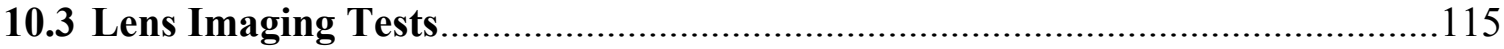

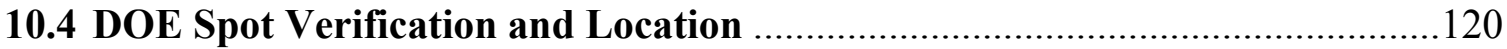

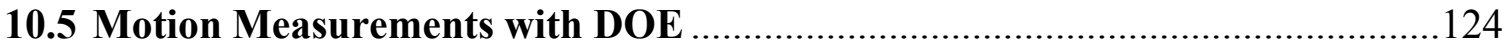

$\begin{array}{lr}\text { Conclusions } & 116\end{array}$

$\begin{array}{ll}\text { Future Work } & 117\end{array}$

$\begin{array}{lr}\text { Appendix } & 118\end{array}$

$\begin{array}{lr}\text { Bibliography } & 119\end{array}$ 


\section{List of Figures}

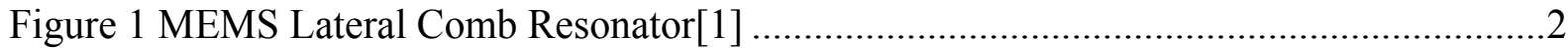

Figure 2 Proposed layout of on-axis integrated monitoring scheme [1] ...................................

Figure 3 Wafer layout of Fresnel DOE lenses [1] ………....................................................

Figure 4 On-axis lens diffraction pattern produced in Mat-Lab[1] ……..................................

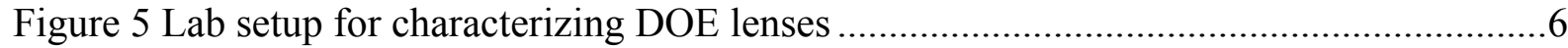

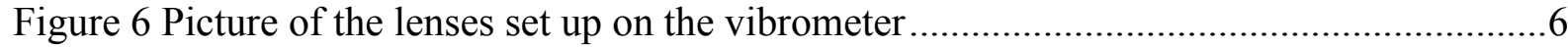

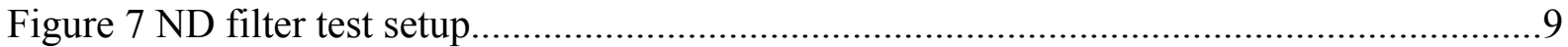

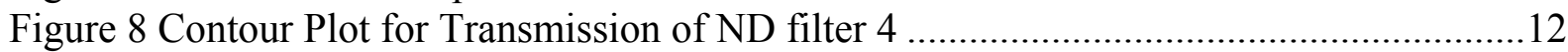

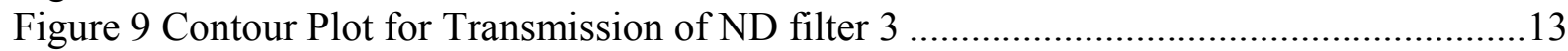

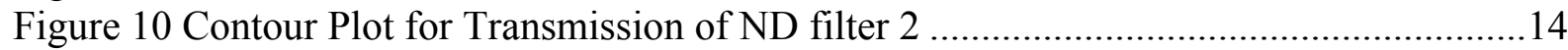

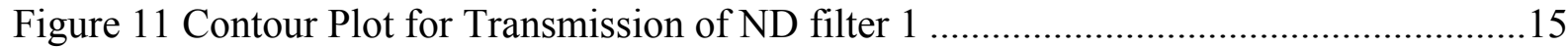

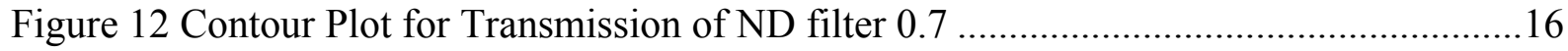

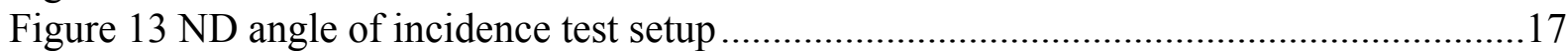

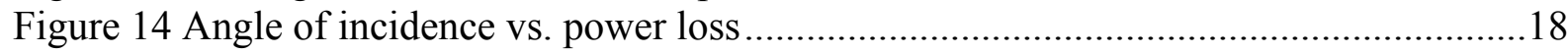

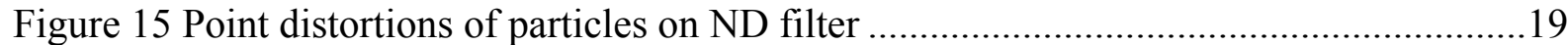

Figure 16 Spectral Response of HeNe Laser from 400-800nm............................................27

Figure 17 Spectral response of $\mathrm{HeNe}$ laser from 628-640nm.................................................27

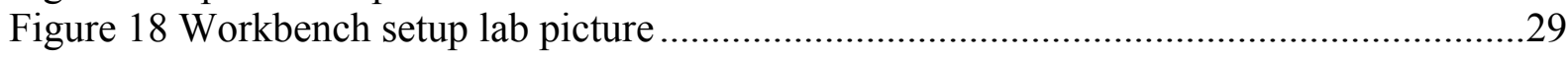

Figure $19 \mathrm{HeNe}$ beam diameter prediction curve and data.......................................................

Figure 20 Difference between 4-Sigma beam diameter measurements and prediction curve...32

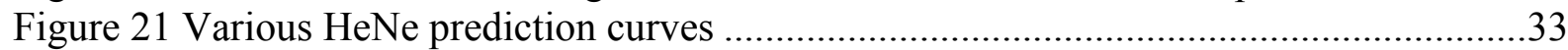

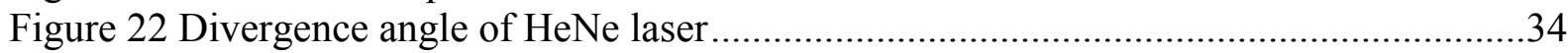

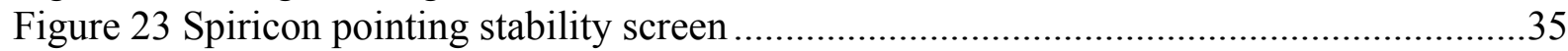

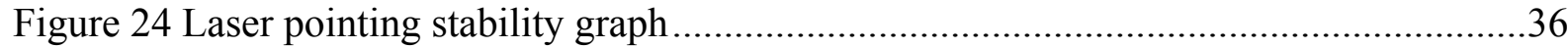

Figure $25 \mathrm{HeNe}$ laser beam sampled profile in Spiricon.........................................................38

Figure 26 2D and 3D picture of HeNe beam at 0.15 meters in Spiricon..................................39

Figure 27 2D and 3D picture of HeNe beam at 0.20 meters in Spiricon..................................39

Figure 28 Drawing explaining how power profile is found..................................................40

Figure 29 Power density profile of HeNe beam at various distances from laser housing ........41

Figure 30 Power density over distance from laser.............................................................42

Figure 31 Power density curve fit....................................................................................42

Figure 32 Breakdown of Borofloat material composition[15] ...............................................45

Figure 33 Borofloat tranmittance vs. IR wavelength [17] ...................................................46

Figure 34 Borofloat transmittance vs. UV wavelength [16] ...................................................46

Figure 35 Borofloat index of refraction vs. wavelength [18] ...............................................4

Figure 36 Setup used to take lens characterization measurements .........................................48

Figure 37 Lens and Microscope Objective alignment ..........................................................49

Figure 38 Ruler markings used to calculate objective magnification .......................................50

Figure 39 Progression of optics theories from simplest (Ray optics) to most complex

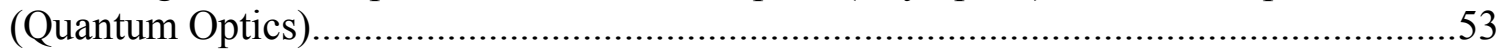

Figure 40 Diagram displaying Fresnel-Huygens principle[8] ................................................56 
Figure 41 Binary and Continuous profiles of a Fresnel Zone Plate.........................................59

Figure 42 Fresnel lens C2R1 (circled in red) was used for tests [1] ......................................63

Figure 43 Designed profile of lens C2R1 created by a Mat-lab program[1] ...........................64

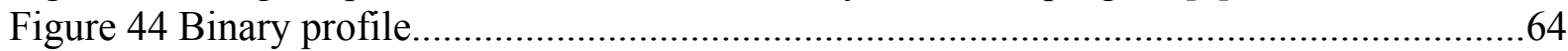

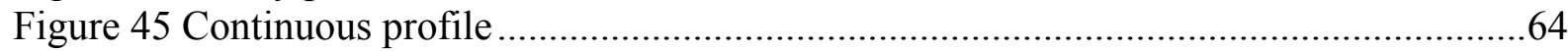

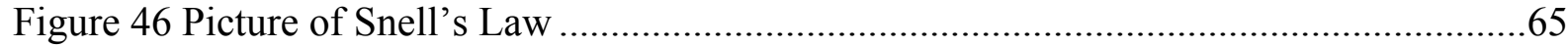

Figure 47 Paraxial Test ...............................................................................................65

Figure 48 Comparison of $13.5 \%$ and 4-sigma methods in..................................................66

Figure 49 Time lapse pictures of beam passing normal to and vertically across lens ...............68

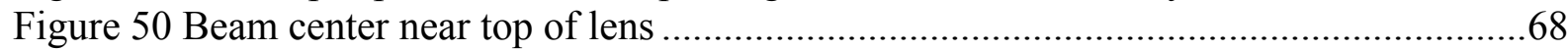

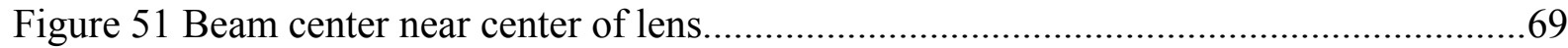

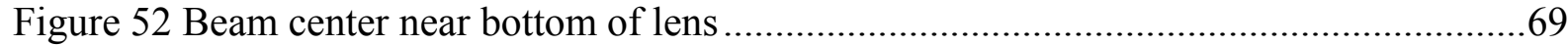

Figure $53 \mathrm{Lab}$ picture of setup used for focal point measurement ..........................................70

Figure 54 Diameter measured with $13.5 \%$ method at $5.385 \mathrm{~mm}$ from lens .............................71

Figure 55 Diameter measured with $13.5 \%$ method at $5.525 \mathrm{~mm}$ from lens .............................71

Figure 56 Diameter measured with $13.5 \%$ method at $5.563 \mathrm{~mm}$ from lens .............................71

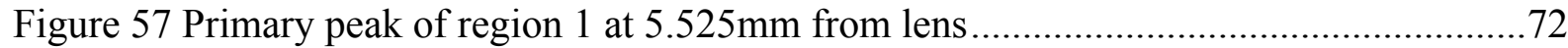

Figure 58 Secondary peak of region 1 at $5.658 \mathrm{~mm}$ from lens............................................73

Figure 59 Lens pointing stability measurements ...................................................................

Figure 60 Drawing of lens and laser spot size difference.....................................................74

Figure 61 Drawing of lens and laser size difference seen in the test setup ..............................75

Figure 62 Aperture used to limit incident light from lens onto CCD camera............................76

Figure 63 Lens focal point spot used to calculate power density .............................................77

Figure 64 Lens focal point power density profile ..................................................................

Figure 68 Comparison of laser and lens focal point power density profiles ............................78

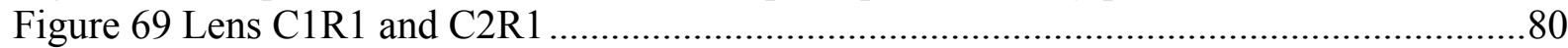

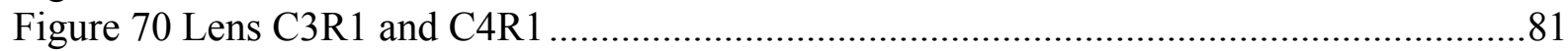

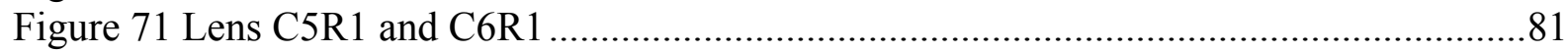

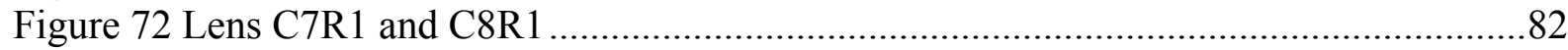

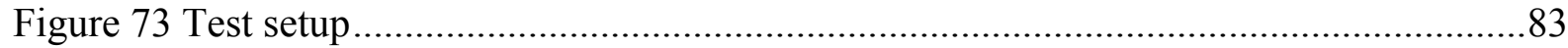

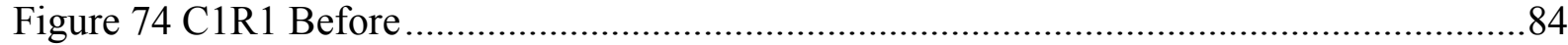

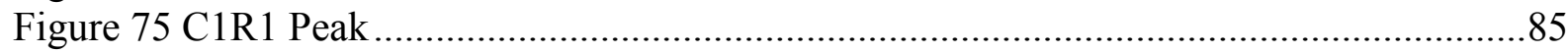

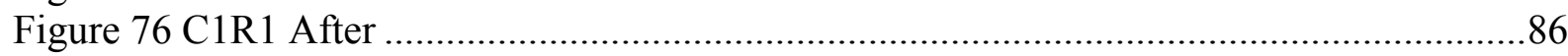

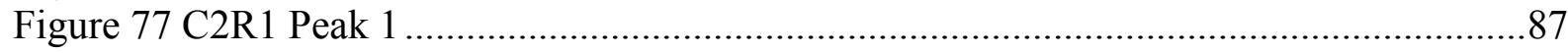

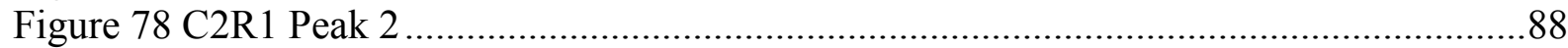

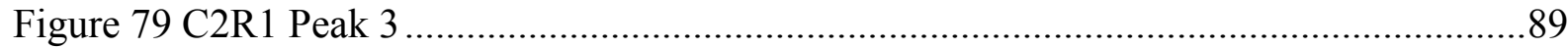

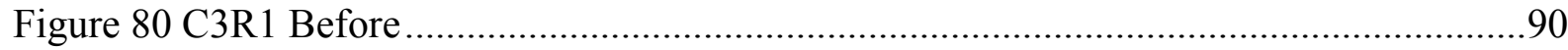

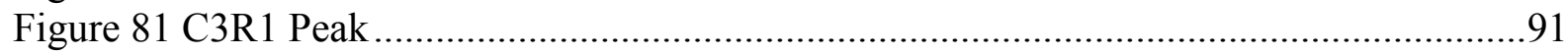

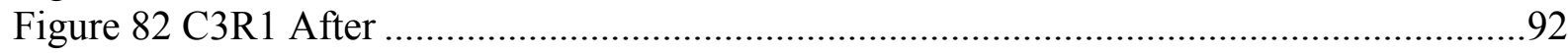

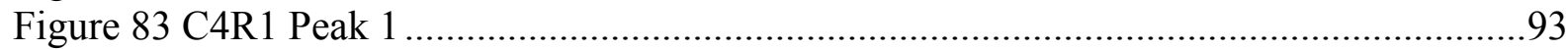

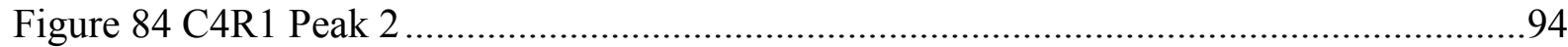

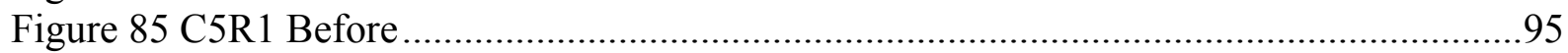

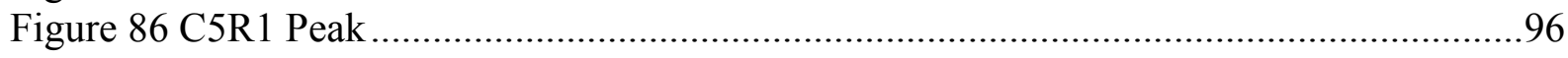

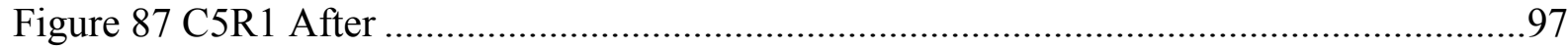

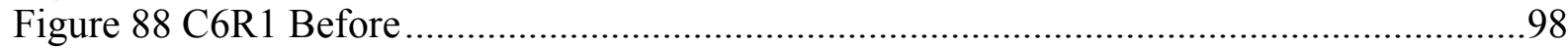

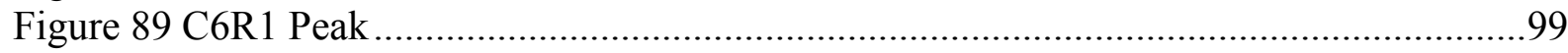




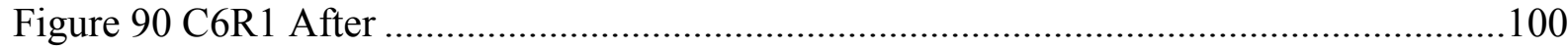

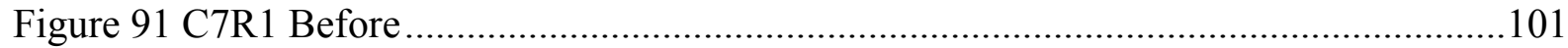

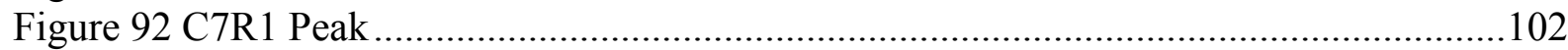

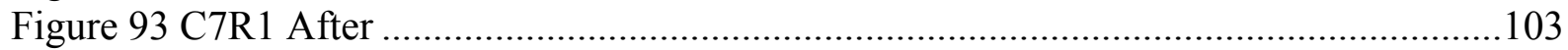

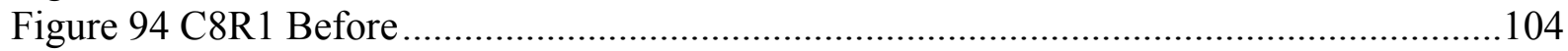

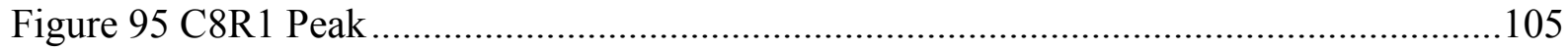

Figure 96 C8R1 After .................................................................................................106

Figure 97 Example diffraction pattern formed from a circular aperture ...............................109

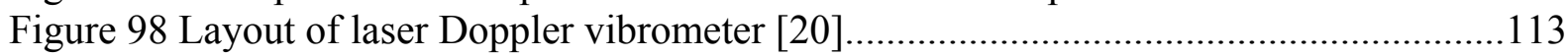

Figure 99 Left to Right: Vibrometer, DOE, and test object setup .........................................114

Figure 100 Fiber terminating piece to allow one fiber operation of vibrometer. ...................114

Figure 101 Lens-Vibrometer test setup ........................................................................115

Figure 102 Layout of images seen with DOE in vibrometer setup......................................116

Figure 103 Sample lateral comb resonator contact pad viewed at 20x with the vibrometer

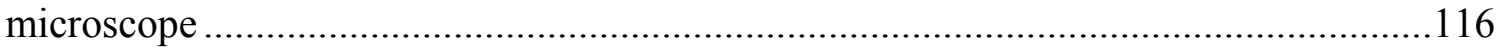

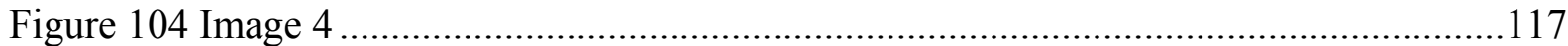

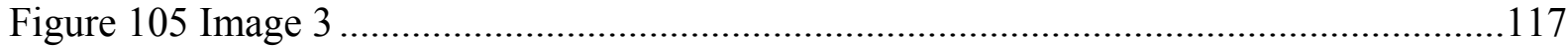

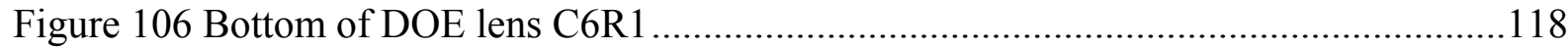

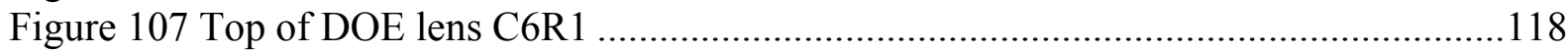

Figure 108 Image 2 reflection of lens ..........................................................................119

Figure 109 Image 1 primary focal point of lens C6R1 ......................................................119

Figure 110 Random device used to determine spot placement as seen with the vibrometer..122 Figure 111 Image 3 showing same placement of the laser spot on the sample using the DOE.

Figure 112 Location of the laser spot from the vibrometer on the DOE. ..............................123

Figure 113 Image 1(inverted) showing same location of laser spot on the sample using the DOE.

Figure 114 Test sample as seen through the vibrometer only. ...............................................125

Figure 115 Displacement measured with vibrometer only over 3-4 kilohertz. ......................125

Figure 116 Displacement measured with vibrometer only over 3.4-3.6 kilohertz. ................126

Figure 117 Background noise $(2.5 \mathrm{pm})$ measured with vibrometer only.............................126

Figure 118 Displacement measured at Image 3 location using DOE from 3-4 kilohertz.......126

Figure 119 Displacement measured at Image 3 location using DOE from 3.4-3.6 kilohertz.127

Figure 120 Displacement measured at Image 1 location using DOE from 3-4 kilohertz.......127

Figure 121 Displacement measured at Image 1 location using DOE from 3.4-3.6 kilohertz. 128

Figure 122 Background noise (3.9pm) observed at Image 1 location with no excitation of device. 


\section{List of Tables}

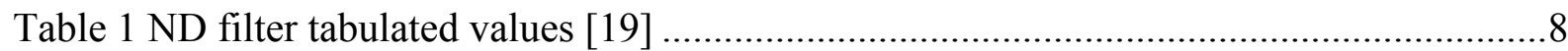

Table 2 ND filter measured transmission values ........................................................... 10

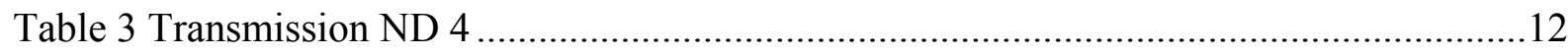

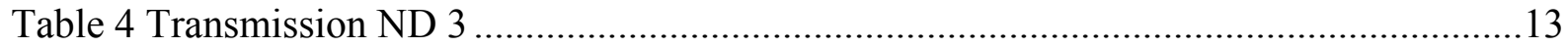

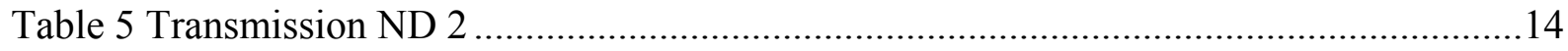

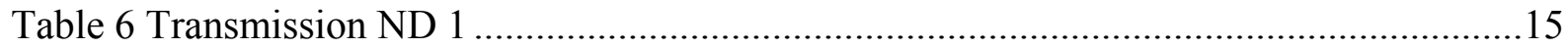

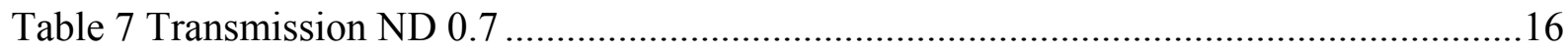

Table 8 Power loss and associated angle of incidence ........................................................ 18

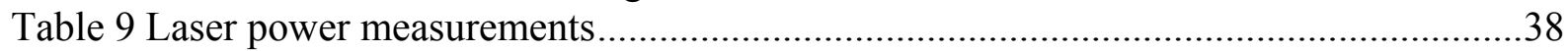

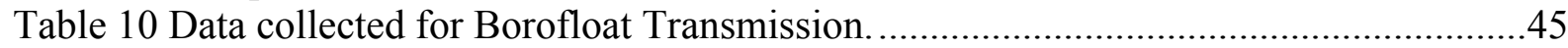

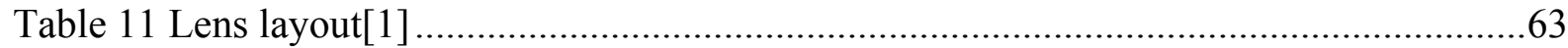

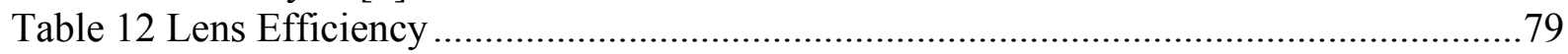

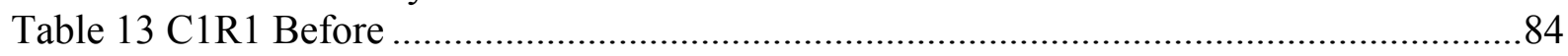

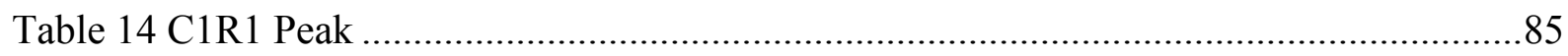

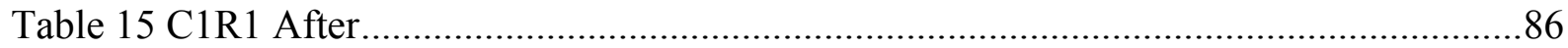

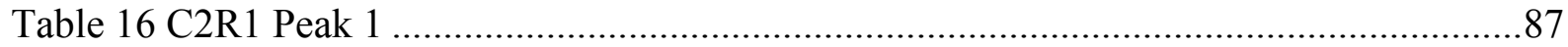

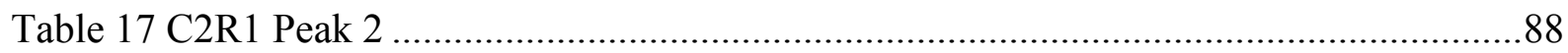

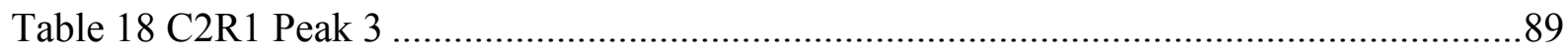

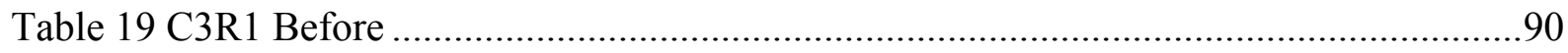

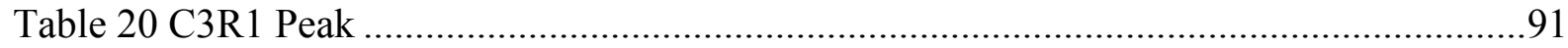

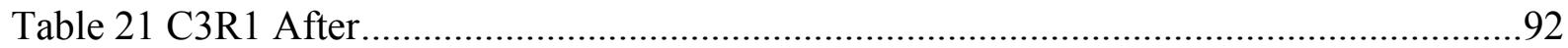

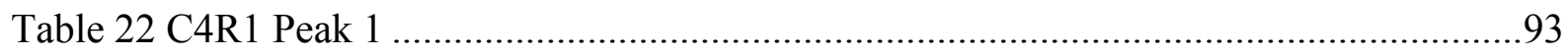

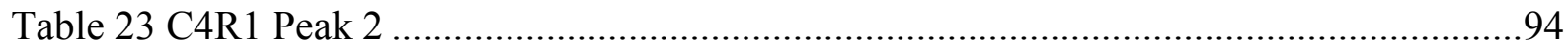

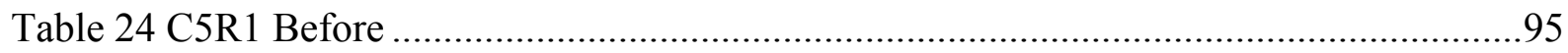

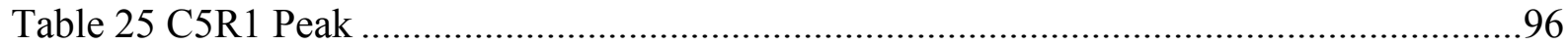

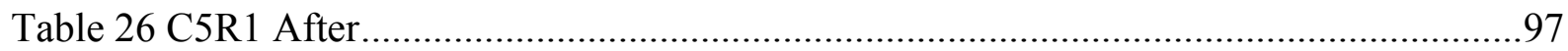

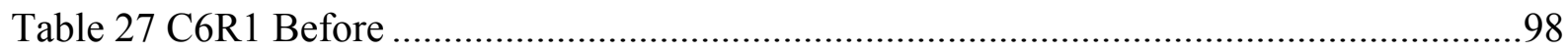

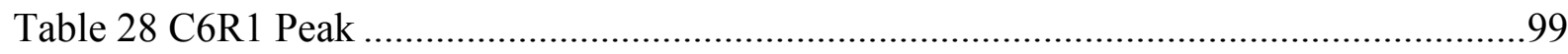

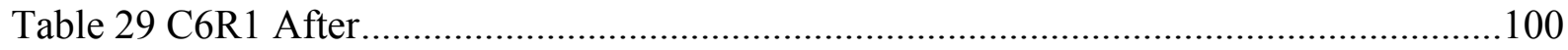

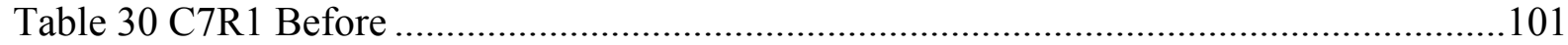

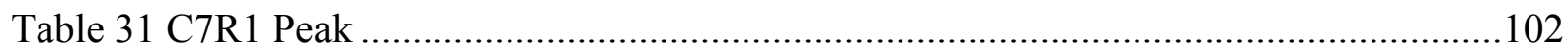

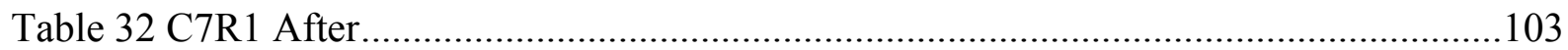

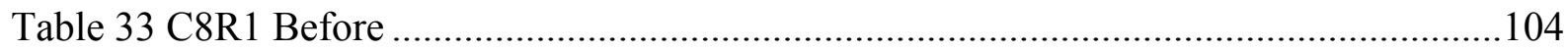

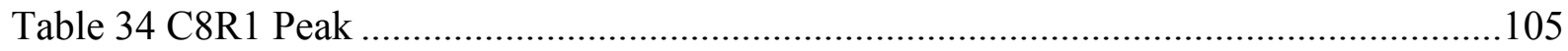

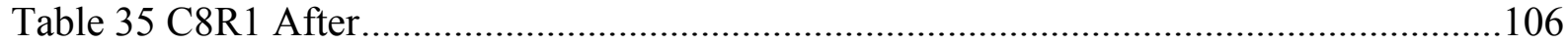

Table 36 Summary of Row 1 on-axis lens characterization .............................................107

Table 37 Tabulated distances and focal ranges of images observed with C6R1 .................120

Table 38 Spot size, Vibrometer, and Ruler measurements ................................................120

Table 39 Vibrometer displacement of piezoelectric device............................................128 


\section{Chapter 1}

\section{Introduction}

\subsection{MEMS and Device Monitoring Needs}

Micro Electro Mechanical Machines (MEMS) are mechanical devices that range in size from a few microns to a few hundred microns. They often can be implemented to perform the same functions as their large scale counterparts, drastically reducing the space requirements for these applications. They can also be used for new applications since their small size can make them more sensitive, more accurate, and operate in a more extended capacity than their macro scaled counterparts. These devices can be used in numerous applications such as accelerometers, airbag deployment systems, drug delivery systems, gyroscopes, and micro gear systems where these characteristics are advantageous.

Critical systems in which MEMS devices are used depend upon the reliability, predictability, and control of these devices. It is necessary to know not only if the device is functioning but how well it is functioning and when it is about to fail. Their small size makes this very difficult to determine. Traditional methods of detecting the operational status of devices using electronic means could be done but can alter the operation of the devices or even damage them. Optical methods of probing these devices can and do provide a non-invasive means to determine their operational status. This control and predictability is being achieved in labs by use of laser Doppler vibrometer systems. These large systems make optical testing of MEMS possible, yet impractical for monitoring in real applications. A vibrometer system has three main components: control and analysis hardware, a laser and fiber delivery system, and a focusing system. Each of these components can be reduced in size to allow a more integrated form of optical monitoring. The ideal situation is to reduce this bulky setup, occupying 6 or more square feet of space, to the size of a circuit board. Realization of 
miniaturization and continuous on-chip optical monitoring of device status would give MEMS devices a wider range of application and provide a more reliable, robust system.

\subsection{Prior MEMS Work}

Previous work $[1,2,3]$ in our group has dealt with MEMS devices, particularly lateral comb resonators. These devices are 300um long, 100um wide, and oscillate laterally when excited with an alternating current. One of these devices is pictured below:
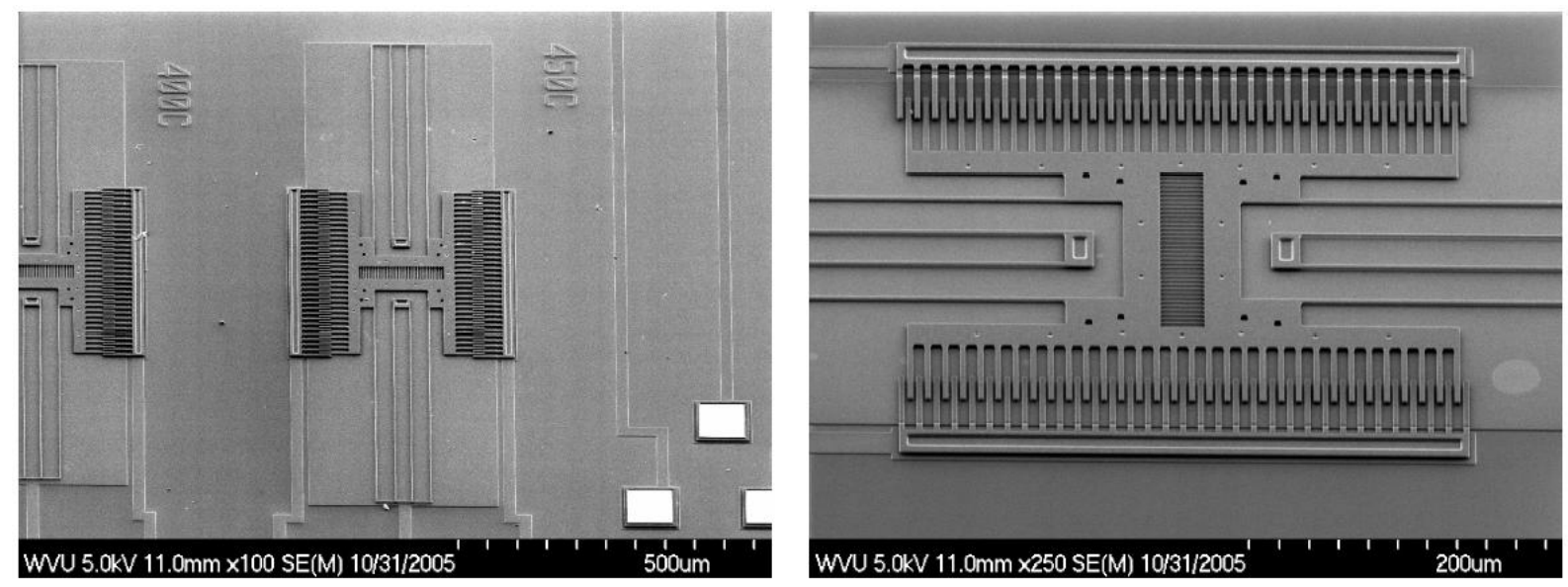

Figure 1 MEMS Lateral Comb Resonator[1]

The inner fingers of the device are held at some set DC voltage $\mathrm{V}$, while the outer fingers are excited with an $\mathrm{AC}$ voltage with a magnitude of $\pm \mathrm{V}$. The inner fingers move side to side because of the voltage difference produced by the AC current on the outer fingers. Current and previous work within our group is concentrated on control, fault detection, and integrated optical monitoring of these MEMS devices [1,2,3]. All of those efforts were intended to increase reliability and robustness of theses systems. Much of the control and fault detection aspects of the research have been completed or are very well understood. An incomplete and necessary part of the research in this area is integrated optical monitoring.

Previous research [14] in the group has provided better understanding, control, and fault detection knowledge of MEMS devices. This work was made possible partly through the use of laser Doppler vibrometry which displays the on going need for such a system. An 
integrated form of this system has potential to provide advantages such as: reduced optical monitoring costs, lifetime optical monitoring of MEMS devices, and more widespread use of MEMS due to their increased predictability and control from this system. Integrated monitoring can make MEMS motion detection more portable and realistic to implement in real applications.

\subsection{Previous Integrated Optical Monitoring Work}

The currently used laser Doppler vibrometer system has been used to perform research on MEMS devices. The main driver for the past and current work is to create a lens that will replace the large optical focusing equipment of this system. This is a major step toward the miniaturization of an optical device monitoring system. Requirements for these lenses are: a focused spot diameter comparable to that produced by the vibrometer ( $5 \mathrm{um}$ ), laser beam transfer to and from the sample along the same path, focal range of a few millimeters down to a few hundred micrometers, lens dimensions small enough to be paired for multiple probe beams and packaged with the MEMS die, and operation at the wavelength of the vibrometer system. These requirements come from a combination of vibrometer system specifications and design considerations for a practical integrated lens. It would be difficult to create, align, and package traditional refractive lenses with these dimensions and requirements. An extremely useful lens is one that could be created along with the MEMS device using traditional, well understood micro-fabrication techniques. A Diffractive Optical Element (DOE) lens could be made in this way with the afore mentioned specifications.

Previous research $[1,2,3]$ in the area of integrated diffractive lenses by our group has lead to the modeling, design, and fabrication of Fresnel diffractive optical elements. Below is an image of the proposed on-axis lens scheme [1]. 


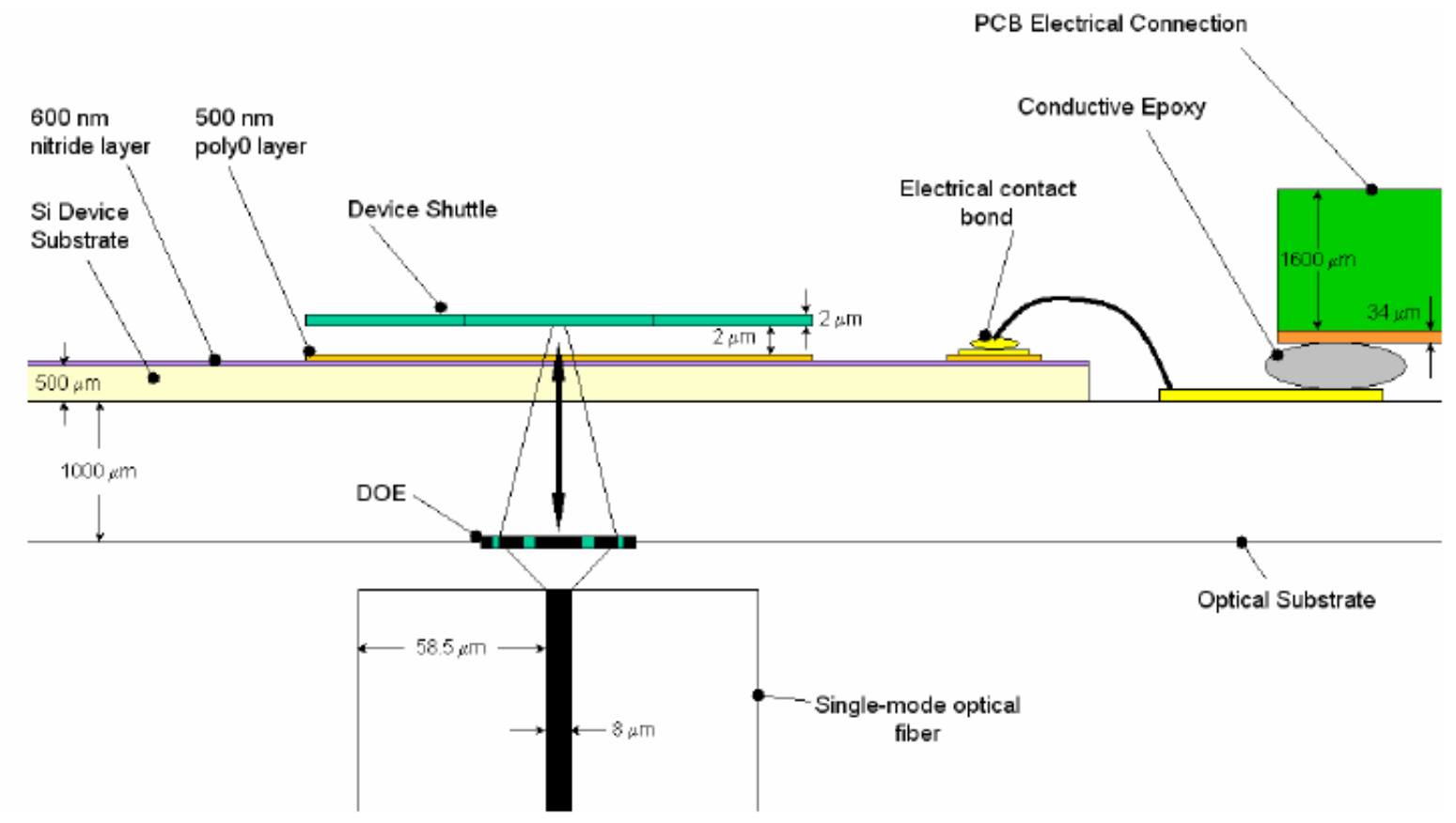

Figure 2 Proposed layout of on-axis integrated monitoring scheme [1].

The device shown here refers to a lateral comb resonator but could ideally be any MEMS device. The incoming laser light is from a fiber input which also captures and returns the reflected laser spot from the device for input to a vibrometer system. The following figures show a conceptual test layout of the diffractive lenses designed within our group [1]: 


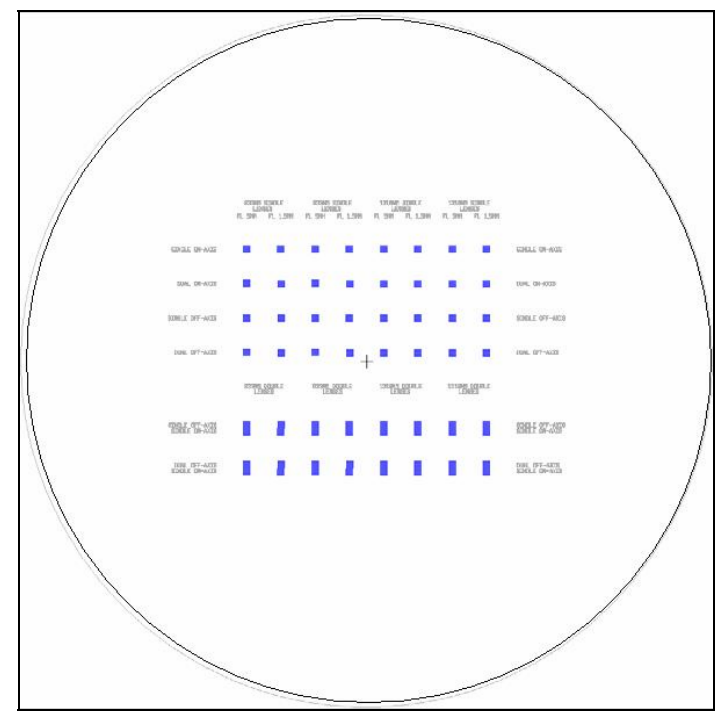

Figure 3 Wafer layout of Fresnel DOE lenses [1]

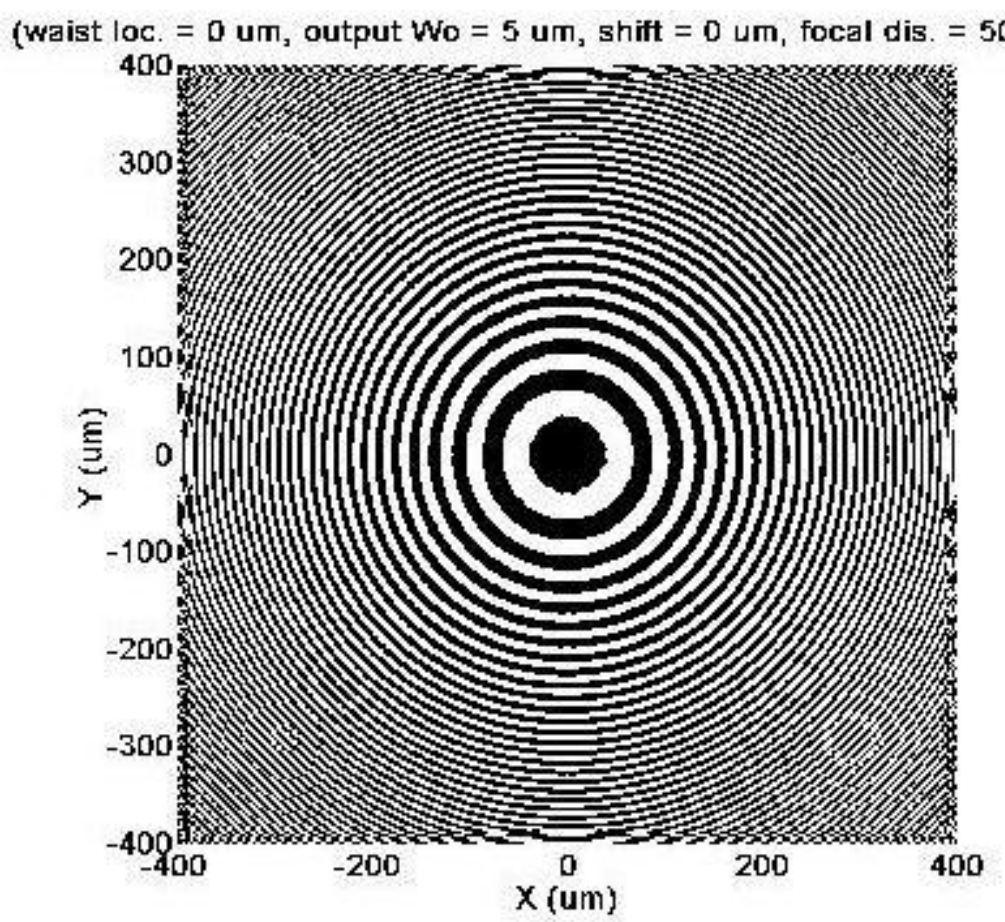

Figure 4 On-axis lens diffraction pattern produced in Mat-Lab[1]

The on-axis lenses are $800 \mathrm{um}^{2}$ and designed to focus a 5 um laser spot to and from the sample, have focal ranges from 1 to $5 \mathrm{~mm}$, be fabricated using photolithography techniques, and operate at the $633 \mathrm{~nm}$ wavelength of the vibrometer system. Half of the lenses were 
designed to operate at the $1310 \mathrm{~nm}$ wavelength, yet appear to perform well when used with $633 \mathrm{~nm}$ laser light. These lenses were designed using Mat-Lab and etched onto a Borofloat wafer using photolithography and wet etching [1].

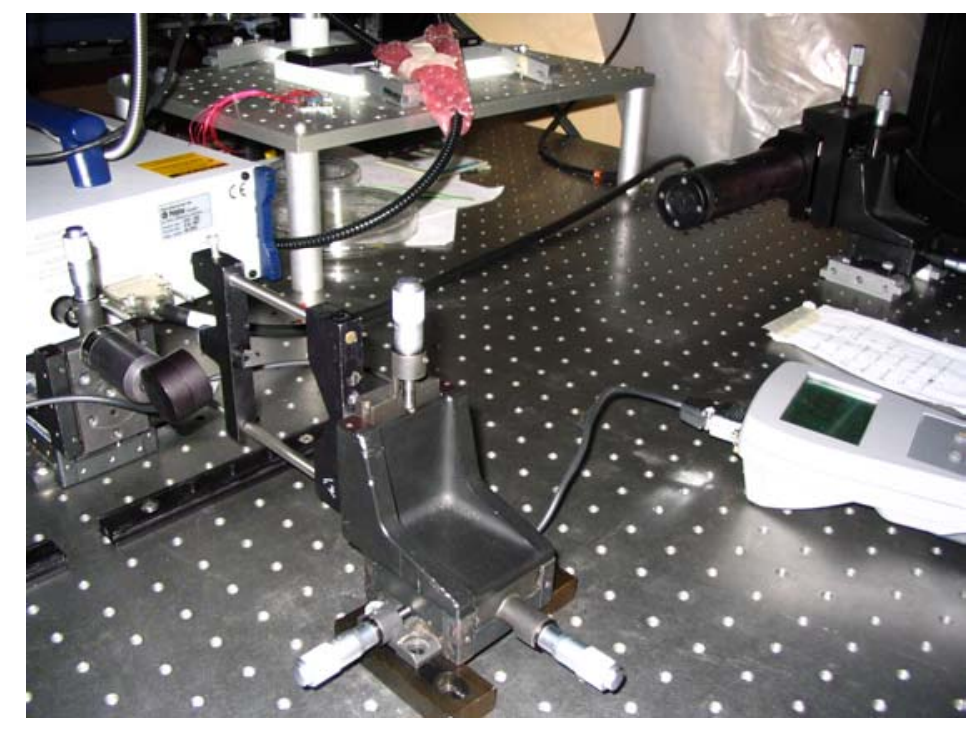

Figure 5 Lab setup for characterizing DOE lenses

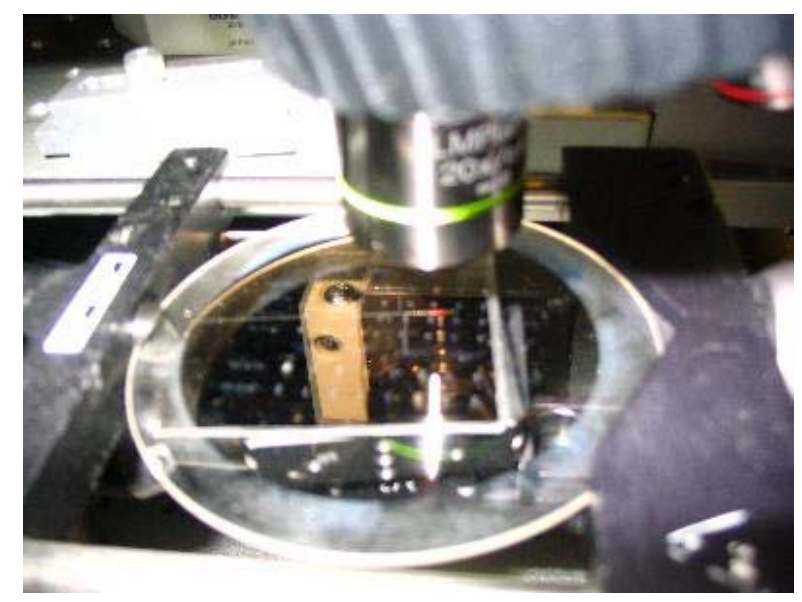

Figure 6 Picture of the lenses set up on the vibrometer

The figure above shows the fabricated lens wafer being used to focus and return light from the laser vibrometer in a successful attempt at motion detection. 


\subsection{Direction and Organization of Thesis}

Prior work left the experimental and theoretical confirmation of the lens operation to be completed as well as confirmation of their usefulness in MEMS motion detection. The research outlined in this thesis determines if the fabricated lenses operate within intended and required parameters, conform to theoretical predictions and models, and can be theoretically and experimentally shown to replace the vibrometer focusing system. The first step to testing the lenses is to understand the behavior of the equipment used for the tests. This necessitates characterization of the laser used on the lenses, the imaging system used, any optical elements used to aid in the testing, and the material on which the lenses were made. Without understanding of the equipment used in testing, effects from one or more of these items may be misinterpreted as an effect produced by the diffractive lenses. Chapter 2 outlines the characterization of Neutral Density filters used on the CCD camera. This camera was used in the laser and lens spot characterization. These filters reduce incident light on the camera so that laser light can be observed without damaging the camera. It should be known if the filters alter the observed laser light in any way. Chapter 3 describes characterization of the $\mathrm{HeNe}$ laser used in the diffractive lens characterization. The laser output should be predictable so the diffractive lens response can be properly determined with it. Chapter 4 explains the characterization of the microscope objective required to magnify and observe the lens spot. The power losses from this device were needed to aid in diffractive lens efficiency calculations. Chapter 5 describes the testing of the borofloat wafer on which the lenses were etched. The loss associated with this material must be accounted for when determining lens efficiency. Its composition must also be understood to determine how it affects the incident laser light when used as a diffractive lens material. Chapter 6 explains the optical theory needed to understand how the lenses and many of the test devices operate. Chapter 7 outlines the theory and modeling used to describe and create the diffractive lenses. Chapter 8 explains the experimental characterization of the lenses. This is needed to determine and confirm such things as: efficiency, signal to noise ratio, spot size, focal length, spot power density, etcetera. Chapter 9 describes the simulation of the lens output as observed before and after the focal point of the lens. These simulations display an extended use of the program and its potential as a diffractive element modeling tool. Chapter 10 discusses the vibrometer tests performed to determine if the lenses can be used to replace the vibrometer optics. 


\section{Chapter 2}

\section{Neutral Density Filter Characterization}

The neutral density filters are $5 \mathrm{~cm}$ square pieces of glass-like material used to attenuate laser power. They are needed to diminish the incident power on the Cohu 4812 CCD camera used to observe the beam. There are six filters with the following designations and tabulated transmissions percentages:

\begin{tabular}{|c|r|}
\hline Filter Number & $\begin{array}{r}\text { Tabulated } \\
\text { \%Tx }\end{array}$ \\
\hline 0.3 & $50 \%$ \\
\hline 0.7 & $20 \%$ \\
\hline 1 & $10 \%$ \\
\hline 2 & $1 \%$ \\
\hline 3 & $0.10 \%$ \\
\hline 4 & $0.01 \%$ \\
\hline
\end{tabular}

Table 1 ND filter tabulated values [19]

The transmission is reported to be uniform between the wavelengths of 400-700nm [35]. The $\mathrm{HeNe}$ laser used has an operating wavelength of approximately $632.9 \mathrm{~nm}$.

\subsection{Test Setup}

The sensor of a handheld power meter (PM100 from Thor Labs) was fixed to the workbench approximately 0.15 meters from the laser face. A background light power measurement was taken as well as the power of the laser without using the filters. Each filter was then placed between the laser and the power sensor by fixing it to the bench using a clamp. The filters were made to be approximately perpendicular to the incident laser by reflecting the laser light 
to a point close to (but not directly into) the laser aperture. Reflecting the beam back into the laser could cause unwanted effects such as inflated, false power readings.

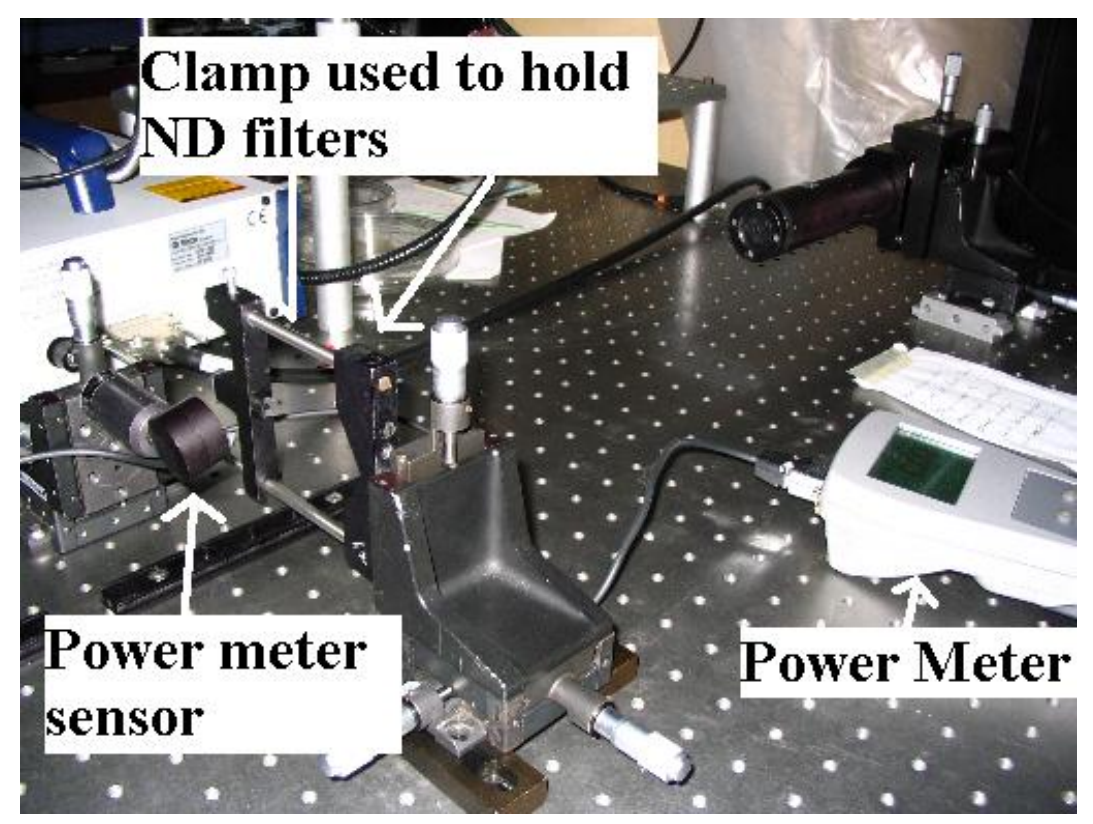

Figure 7 ND filter test setup

An average power measurement was then taken over the course of one minute for each filter. The room lights were turned off during this experiment to reduce the background light levels to approximately $30-33 \mathrm{nW}$. This is important since the light measured through the ND 3 and 4 filters was recorded to be less than the background light levels.

\subsection{Measured Neutral Density Filter Transmission Percentages}

Light power transmission data was collected at nine equally spaced locations over a $0.161 \mathrm{~m}^{2}$ region near the center of each filter. Each point was continuously sampled for approximately one minute. The nine samples and their standard deviations were then averaged and divided by the measured average laser power of $6.827 \mathrm{e}-3 \mathrm{~W}$ to obtain the percentage transmission. The following results were observed: 


\begin{tabular}{|c|c|r|l|}
\hline Filter Number & $\begin{array}{l}\text { Calculated } \\
\text { ND } \\
\text { Designation }\end{array}$ & $\begin{array}{l}\text { Calculated } \\
\% \text { Tx of ND } \\
\text { filters }\end{array}$ & $\begin{array}{l}\text { Standard } \\
\text { Deviation }\end{array}$ \\
\hline 0.3 & 0.20 & $63.76154 \%$ & $0.08972 \%$ \\
\hline 0.7 & 0.83 & $14.62510 \%$ & $6.218 \%$ \\
\hline 1 & 1.21 & $6.21845 \%$ & $0.008676 \%$ \\
\hline 2 & 2.75 & $0.17860 \%$ & $0.00006487 \%$ \\
\hline 3 & 4.09 & $0.00805 \%$ & $0.000008091 \%$ \\
\hline 4 & 5.32 & $0.00048 \%$ & $0.000007679 \%$ \\
\hline
\end{tabular}

Table 2 ND filter measured transmission values

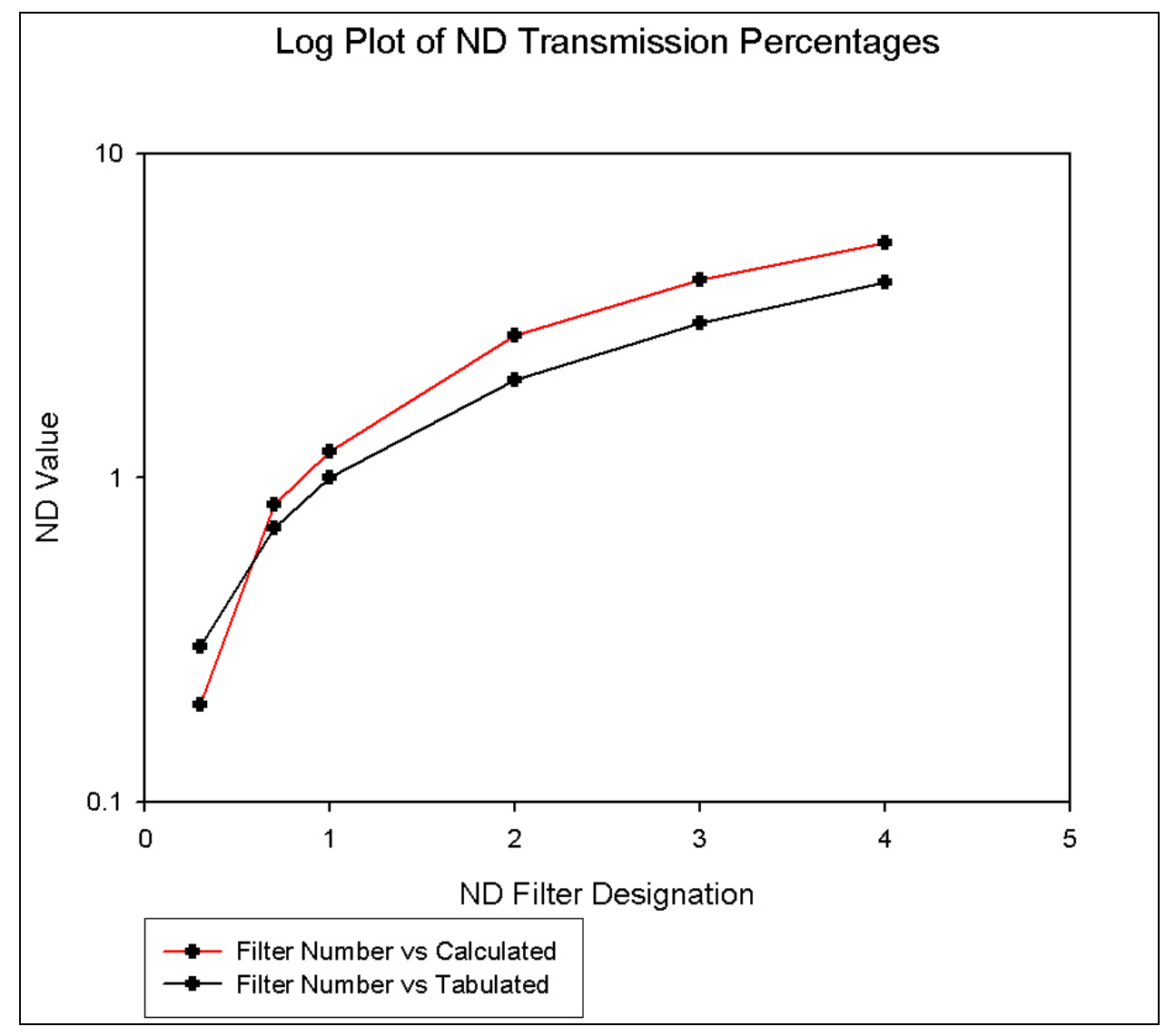

Figure 7.1 Semi-Log plot of ND filter values

These measurements were performed twice on separate occasions and the same results were obtained. The experimentally calculated transmission percentages vary substantially from the tabulated ones. The logarithm of the percentage is the ND designation. 


\subsection{Uniformity of Neutral Density Light Transmission}

Measurements were later taken to determine whether the light transmission was uniform across the filters. The following figures show contour plots showing transmission gradients through each filter. The filters were approximately normal to the laser beam for theses measurements. The filters were wiped off with a lens cloth and dust was blown off using compressed air. A $0.161 \mathrm{~mm}^{2}$ region was selected near the center of each filter over which nine equally spaced data locations were selected. The micrometers limited the range over which measurements could be taken without altering the test setup. Power was measured continuously for a period of one minute to obtain an average power reading at each data location. Each point was $0.635 \mathrm{~mm}$ apart in both the $\mathrm{x}$ and $\mathrm{y}$ directions beginning at a randomly defined point $(0,0)$ near the canter of the filter. 


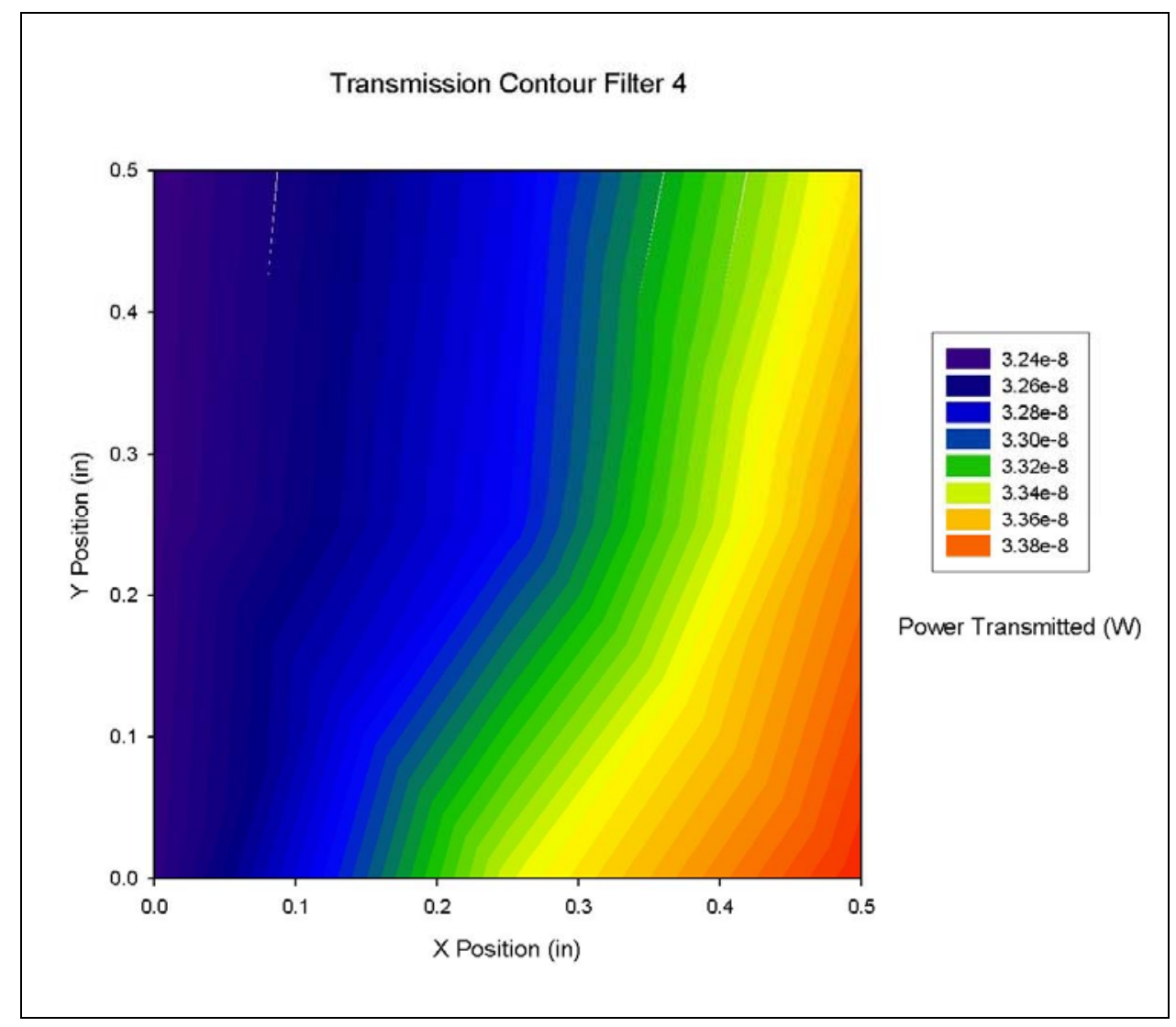

Figure 8 Contour Plot for Transmission of ND filter 4

\begin{tabular}{|l|r|}
\hline & Power (W) \\
Min & $3.236 \mathrm{E}-08$ \\
Max & $3.392 \mathrm{E}-08$ \\
\hline Difference & $1.560 \mathrm{E}-09$ \\
\hline Total Average power & $3.30 \mathrm{E}-08$ \\
Avg Std. & $5.24 \mathrm{E}-10$ \\
\hline
\end{tabular}

Table 3 Transmission ND 4 


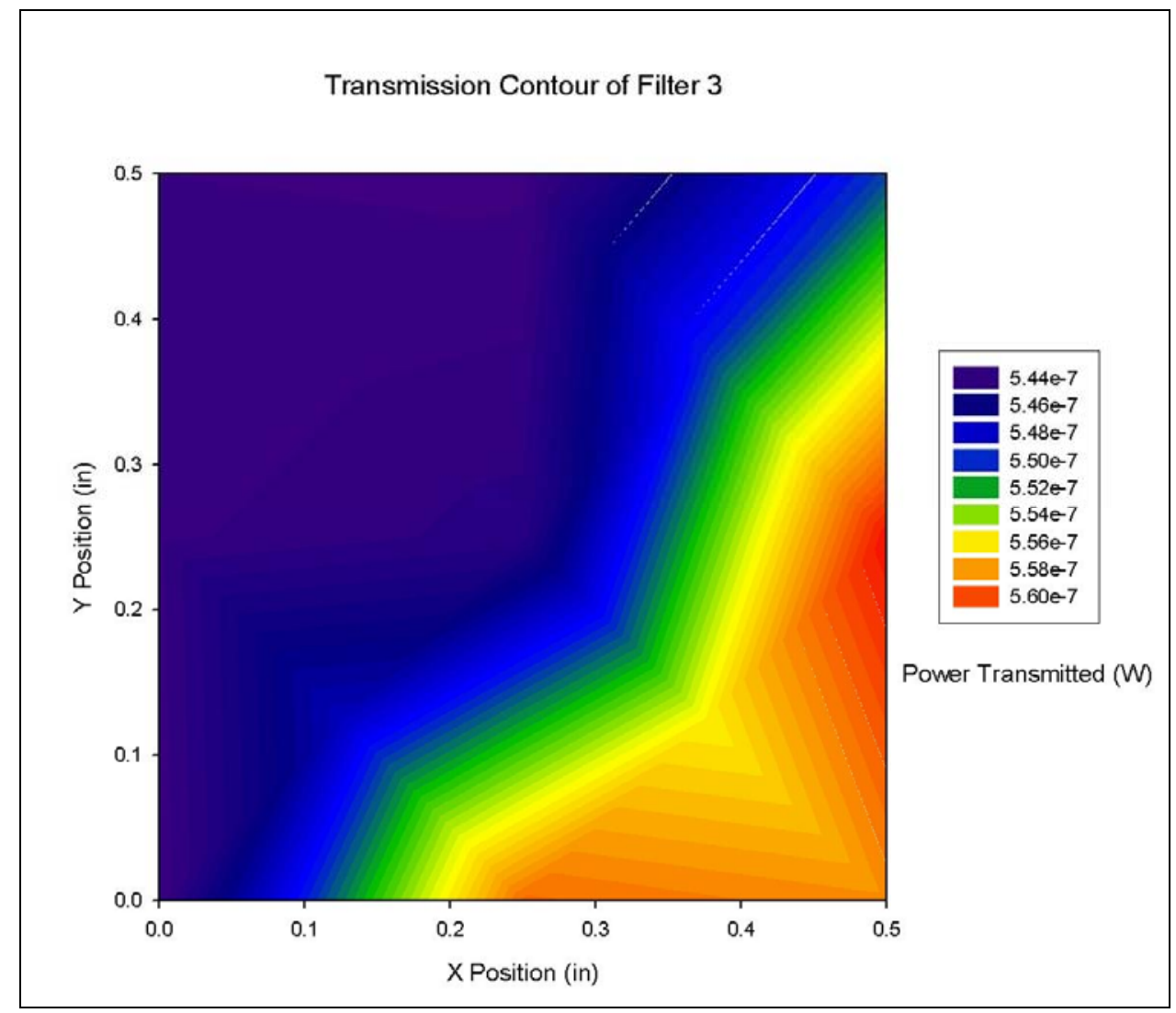

Figure 9 Contour Plot for Transmission of ND filter 3

\begin{tabular}{|l|r|}
\hline Min & Power (W) \\
Max & $5.431 \mathrm{E}-07$ \\
$5.612 \mathrm{E}-07$ \\
\hline Difference & $1.810 \mathrm{E}-08$ \\
\hline Total Average power & $5.50 \mathrm{E}-07$ \\
Avg Std. & $5.52 \mathrm{E}-10$ \\
\hline
\end{tabular}

Table 4 Transmission ND 3 


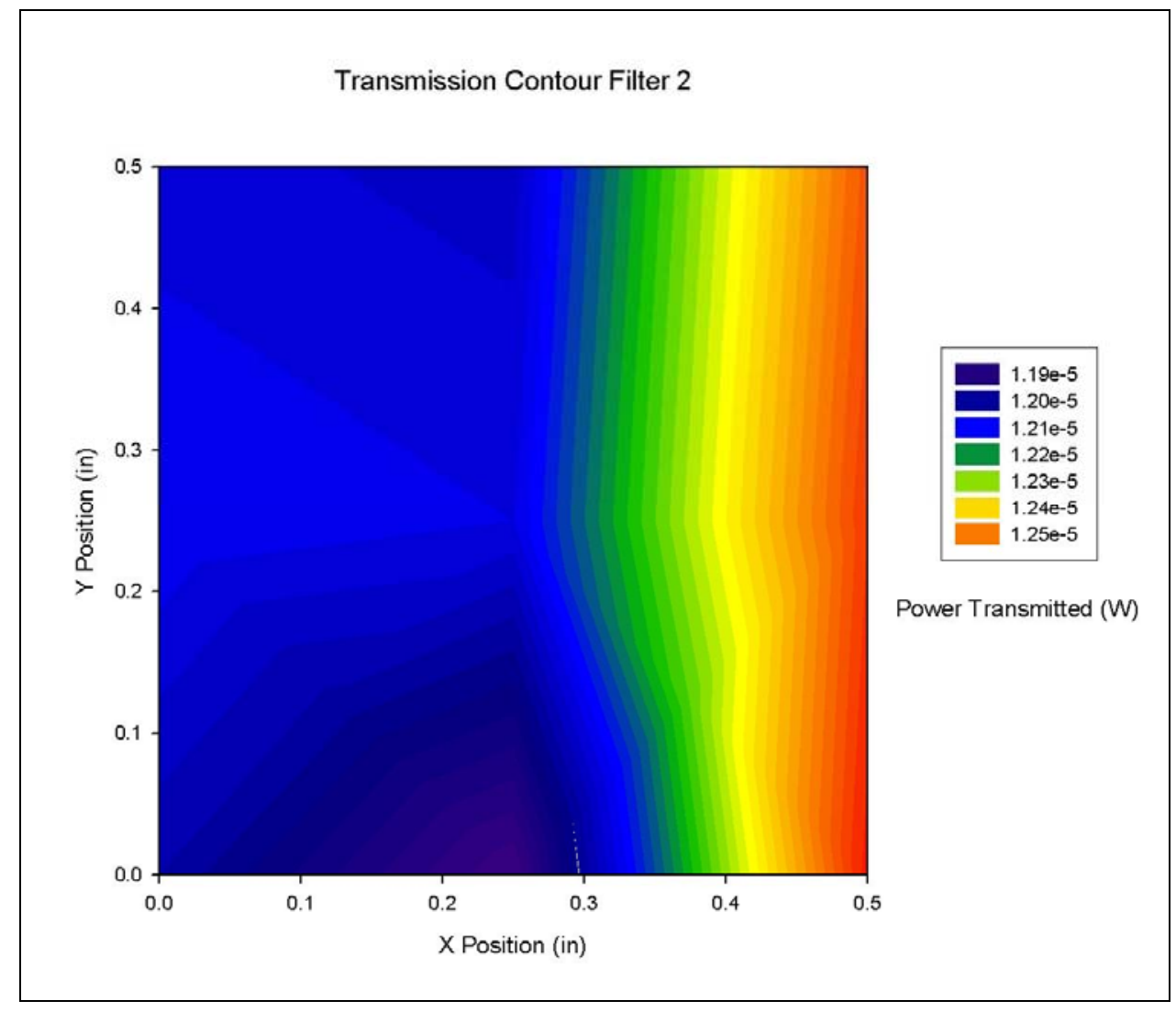

Figure 10 Contour Plot for Transmission of ND filter 2

\begin{tabular}{|l|r|}
\hline Min & Power (W) \\
Max & $1.184 \mathrm{E}-05$ \\
$1.259 \mathrm{E}-05$ \\
\hline Difference & $7.500 \mathrm{E}-07$ \\
\hline Total Average power & $1.22 \mathrm{E}-05$ \\
Avg Std. & $4.43 \mathrm{E}-09$ \\
\hline
\end{tabular}

Table 5 Transmission ND 2 


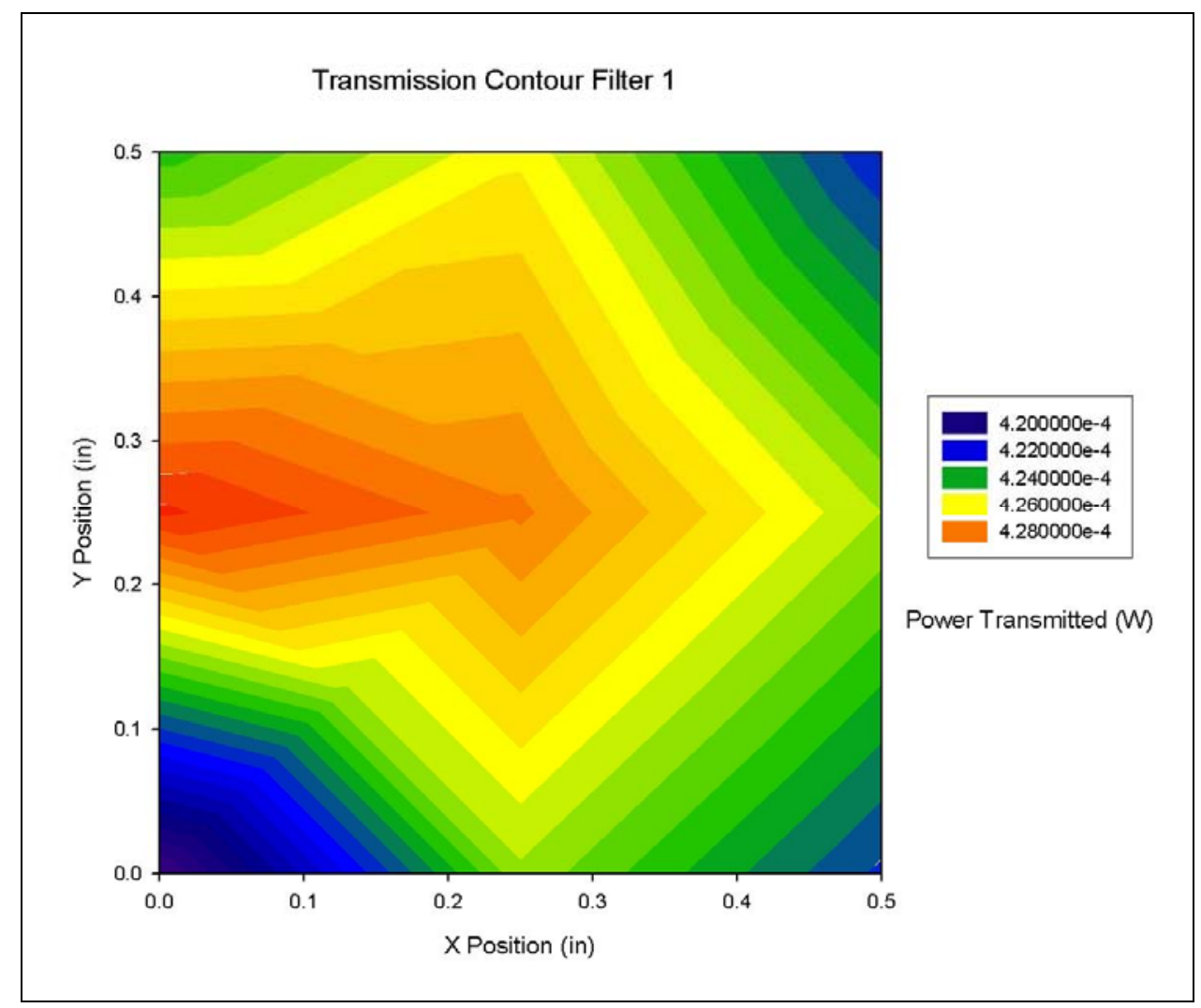

Figure 11 Contour Plot for Transmission of ND filter 1

\begin{tabular}{|l|r|}
\hline \multirow{3}{*}{ Min } & Power (W) \\
\cline { 2 - 2 } Max & $4.187 \mathrm{E}-04$ \\
\cline { 2 - 2 } & $4.289 \mathrm{E}-04$ \\
\hline Difference & $1.020 \mathrm{E}-05$ \\
\hline Total Average power & $4.25 \mathrm{E}-04$ \\
Avg Std. & $1.52 \mathrm{E}-07$ \\
\hline
\end{tabular}

Table 6 Transmission ND 1 


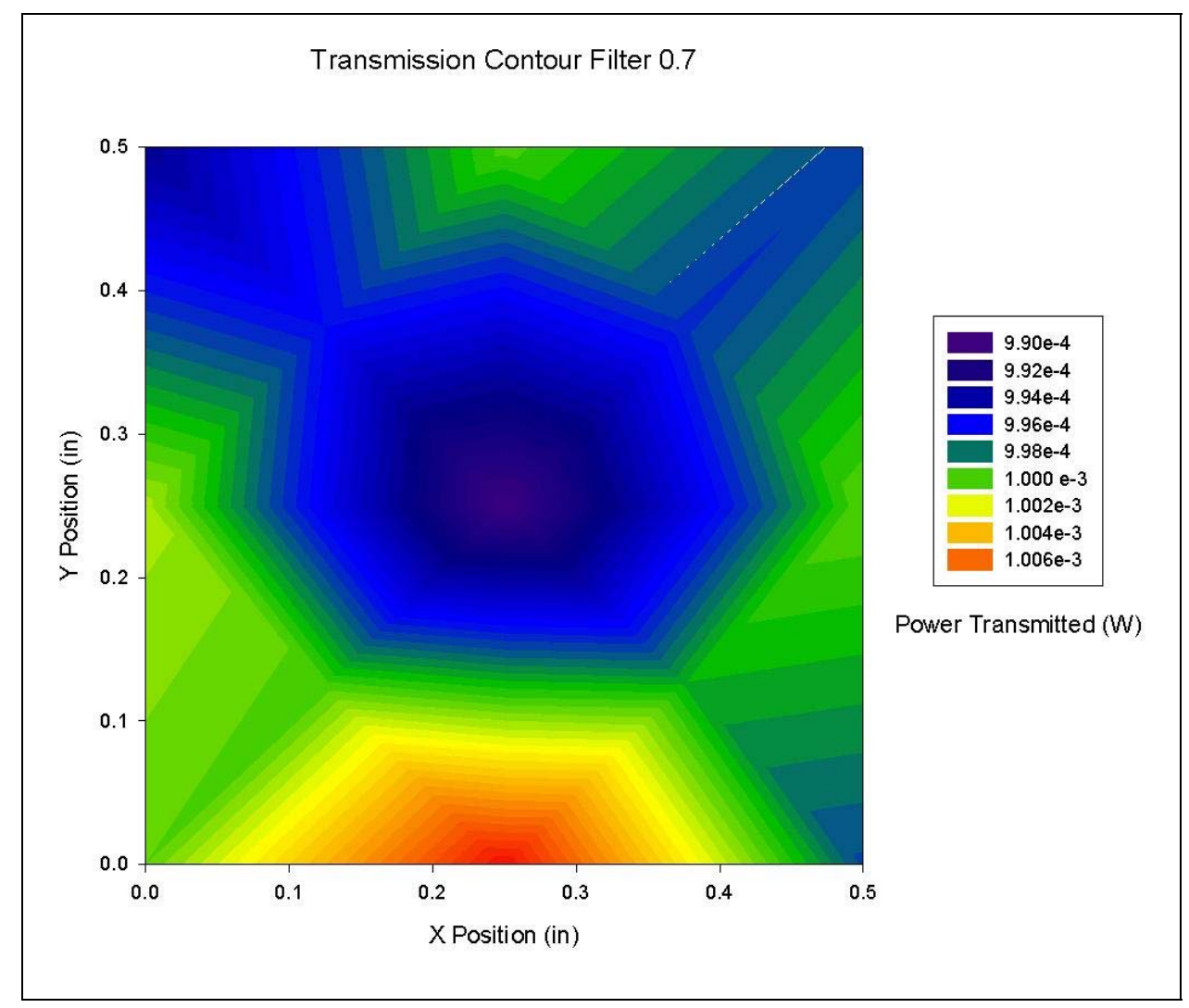

Figure 12 Contour Plot for Transmission of ND filter 0.7

\begin{tabular}{|l|r|}
\hline \multirow{2}{*}{ Min } & Power (W) \\
\cline { 2 - 2 } Max & $9.900 \mathrm{E}-04$ \\
\cline { 2 - 2 } & $1.008 \mathrm{E}-03$ \\
\hline Difference & $1.800 \mathrm{E}-05$ \\
\hline Total Average power & $9.98 \mathrm{E}-04$ \\
Avg Std. & $5.92 \mathrm{E}-07$ \\
\hline
\end{tabular}

Table 7 Transmission ND 0.7 


\subsection{Transmission Through Neutral Density Filters for Non-Normal Incidence}

The next item investigated was the change in transmission due to a change in the angle of incidence of the laser on the filter. This implements the use of the law of reflection which states that the angle of incidence is equal to the angle of reflection. The same equipment setup outlined above was used to gather the data. The top of the lens clamp was rotated towards the laser until each of three designated power drops of $1 \%, 5 \%$, and $10 \%$ were observed. The distance of the reflected spot on the bench was taken with respect to the laser aperture. The laser aperture was measured to be $0.635 \mathrm{~m}$ from the bench surface and $0.356 \mathrm{~m}$ from the center of the filter. The spot position and laser aperture height were used to determine the angle between the incident and reflected beam. The angle of incidence is half of this number.

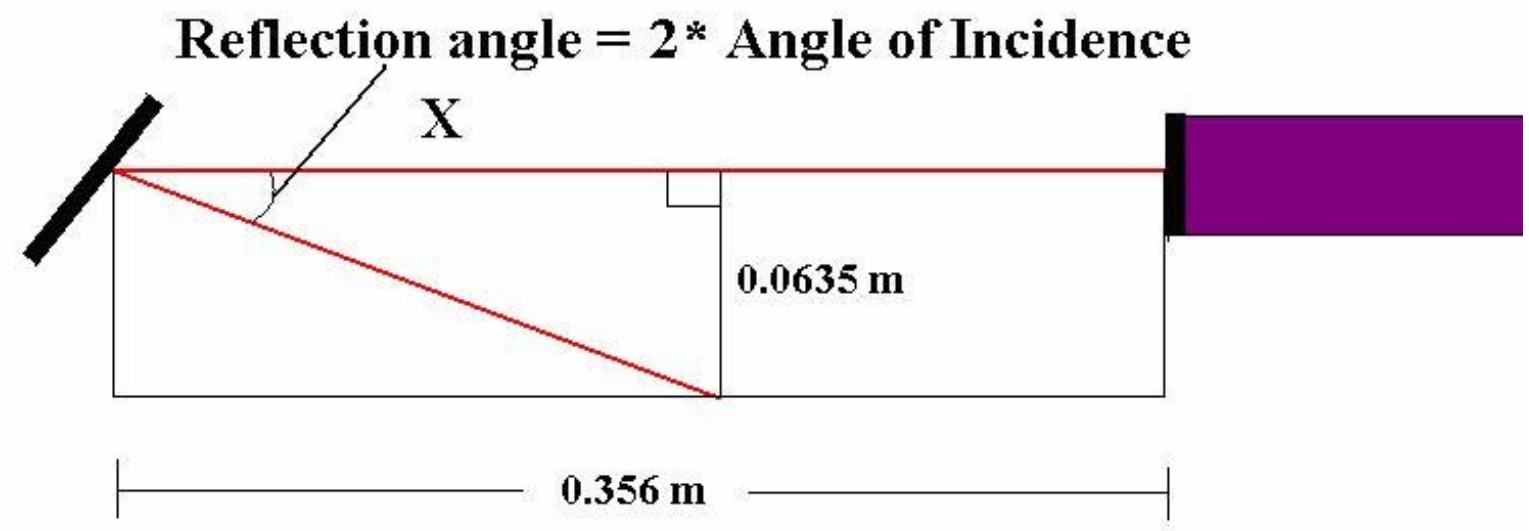

Figure 13 ND angle of incidence test setup

Figure 13 depicts the test setup used to determine the angle of incidence for the ND filters. The total reflection angle was found by using:

(1) $\quad$ Angle $=\arctan (2.5 / \mathrm{X})$

This angle was converted to degrees and divided by two to obtain the angles of incidence listed in Table 8 . The distance $\mathrm{X}$ was obtained by subtracting the spot measurement from 
$0.356 \mathrm{~m}$. This all assumes that the lenses are each a planar, homogeneous, and linear medium.

\begin{tabular}{|c|c|c|c|c|}
\hline $\begin{array}{l}\text { ND Filter } \\
\text { Number }\end{array}$ & $\begin{array}{l}\text { Power } \\
\text { loss }\end{array}$ & $\begin{array}{l}\text { Angel of } \\
\text { Incidince (deg) }\end{array}$ & $\begin{array}{l}\text { Spot Distance } \\
\text { From Laser (m) }\end{array}$ & $\begin{array}{l}\text { Starting Power } \\
\text { (W) }\end{array}$ \\
\hline \multirow[t]{3}{*}{1} & $1 \%$ & 6.402 & 0.089 & \multirow[t]{3}{*}{$4.54 \mathrm{E}-04$} \\
\hline & $5 \%$ & 18.26 & 0.270 & \\
\hline & $10 \%$ & 29.52 & 0.318 & \\
\hline \multirow[t]{3}{*}{2} & $1 \%$ & 4.065 & -0.089 & \multirow[t]{3}{*}{ 1.29E-05 } \\
\hline & $5 \%$ & 11.98 & 0.213 & \\
\hline & $10 \%$ & 18.78 & 0.273 & \\
\hline \multirow[t]{3}{*}{3} & $1 \%$ & 4.123 & -0.083 & \multirow[t]{3}{*}{$5.66 \mathrm{E}-07$} \\
\hline & $5 \%$ & 9.827 & 0.178 & \\
\hline & $10 \%$ & 14.53 & 0.241 & \\
\hline
\end{tabular}

Table 8 Power loss and associated angle of incidence

The negative distances indicate the spot was reflected to a point behind the laser aperture.

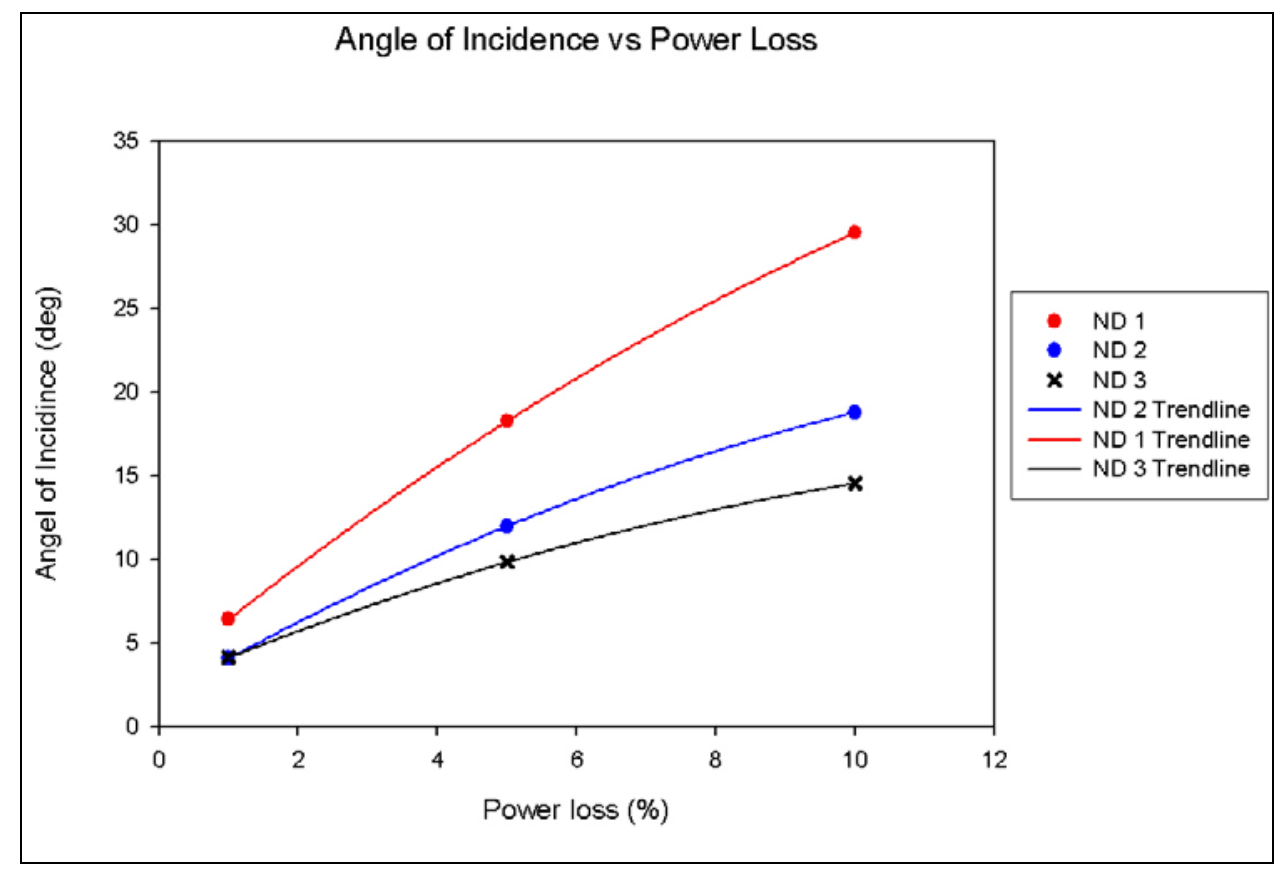

Figure 14 Angle of incidence vs. power loss

Figure 14 shows negative second order polynomial trend lines for the angle of incidence verses percent power loss of ND filters 1,2 , and 3 . The error bars indicate a maximum 
possible \pm 5 degree error in the calculated angles. It was assumed that the distance $\mathrm{X}$ in Figure 13 was measured from the center of the filter. Some error can occur if the incident beam is not placed on the pivot point of the filter. If the beam is placed above the pivot point, the laser-aperture distance will be smaller since the top of the filter is rotated toward the laser. If the incident beam spot is placed below the pivot point, the laser-filter distance will be larger. An angle of 45 degrees could alter the laser-filter distance by $\pm 17.9 \mathrm{~mm}$ at the outer edge of the filter which can produce $\mathrm{a} \pm 2$ degree difference in the incident angle. This is believed to be an upper bound to the error since all calculated angles are less than 45 degrees and the incident beam was close to the center of each filter.

\subsection{Point Distortions from Particulate Matter on Filters}

Particulate matter on ND filters caused a point source diffraction pattern. The effects of this were determined to be negligible in comparison to the power used from the laser. The following pictures show these point distortions caused by dust on the ND filters. A microscope objective was used to spread out the laser light so it could both illuminate the filter and reduce incident power on the camera sensor so as to not damage the camera. This light spreading allowed the inspection of each filter individually without using the other filters to reduce the laser power. This incident light used to observe the dust produced point distortions is several orders of magnitude below that of the focused spot from the DOE.

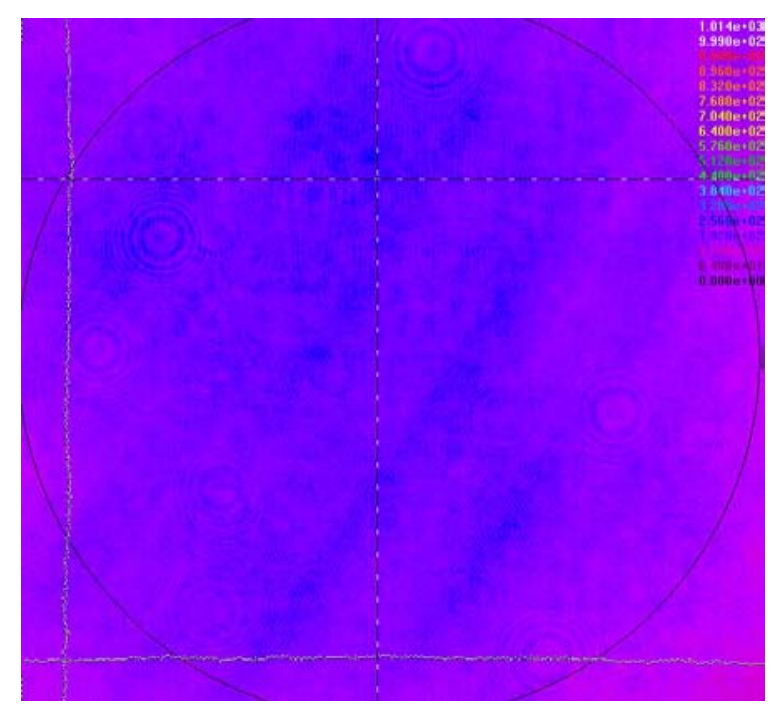

Figure 15 Point distortions of particles on ND filter 


\section{Chapter 3}

\section{HeNe Laser Characterization}

Several things must be known to characterize the laser used: spectral output, waist location and size, angular divergence, pointing stability, power dissipation over distance, in plane power distribution, and peak power. Knowledge of the laser output will provide a basis for comparison when studying the effects of the Fresnel lenses on the laser beam later in the thesis.

The laser used was a class IIIb 10mW HeNe laser model 105-2 from Spectra Physics. Using a class IIIb laser requires precautions to prevent eye damage such as those outlined in appendix A. Even a laser of this relatively low power can cause eye damage if viewed either directly or through specular reflection (such as from a mirror-like surface).

\subsection{Gaussian Beam Theory and Gas Lasers}

The following discussion is adapted from previous optics texts[4,8]. The Gaussian beam is useful in optics in that it is spatially confined and has minimal angular spread which makes it a paraxial wave. Any such wave that makes small angles with respect to the wave front normal is a paraxial wave and must satisfy the paraxial Helmholtz equation. Satisfying this equation is sufficient to identify an electromagnetic phenomenon as a traveling electromagnetic wave. The following is the paraxial Helmholtz equation[4]:

$$
\begin{aligned}
& \nabla_{T}^{2}-j 2 k \frac{\partial A}{\partial z}=0 \\
& \nabla_{T}^{2}=\frac{\partial}{\partial x^{2}}+\frac{\partial}{\partial y^{2}}
\end{aligned}
$$




\subsubsection{Expression for Gaussian Beam}

A paraxial wave is a plane wave that is modulated by a complex envelope which varies slowly as a function of position[4].

$$
\mathrm{U}(\mathbf{r})=\mathrm{A}(\mathrm{r}) \exp (-\mathrm{jkz})
$$

The complex envelope A(r) must satisfy the paraxial Helmholtz equation. A simple solution to the paraxial Helmholtz equation yields a paraboloidal wave[4].

$$
\begin{aligned}
& \mathrm{A}(\overline{\mathrm{r}})=\left(\frac{\mathrm{A}_{1}}{\mathrm{z}}\right) \exp \left(-\mathrm{jk} \frac{\rho^{2}}{2 z}\right) \\
& \rho^{2}=x^{2}+y^{2}
\end{aligned}
$$

The Gaussian beam can be obtained by exchanging $\mathrm{z}$ for $\mathrm{q}(\mathrm{z})=\mathrm{z}-\varepsilon$ into the paraboloidal wave equation, causing a shifted version of the paraboloidal wave. This shifted version is also a solution to the paraxial Helmholtz equation. Making the $\varepsilon$ purely imaginary provides the complex envelope of the Gaussian beam[4].

$$
\begin{aligned}
& A(\bar{r})=\frac{A_{1}}{q(z)} \exp \left[-j k \frac{\rho^{2}}{2 q(z)}\right] \\
& q(z)=z+j z_{0}
\end{aligned}
$$

The Rayleigh range is $\mathrm{z}_{0}$. This range defines the distance from the beam waist $\mathrm{W}_{0}$ to the location where the beam radius has a value of $\sqrt{ }(2) \mathrm{W}_{0}$.

The complex envelope is separated in amplitude and phase components by defining the two new functions wavefront radius of curvature $\mathrm{R}(\mathrm{z})$ and beam width $\mathrm{W}(\mathrm{z})$ [4]:

$$
\frac{1}{q(z)}=\frac{1}{R(z)}-\frac{j \lambda}{\pi W^{2}(z)}
$$


Equations 2, 4, and 5 combine to give the complex amplitude of the Gaussian beam[4]

$$
\mathrm{U}(\mathrm{r})=\mathrm{A}_{0} \frac{W_{0}}{W(z)} \exp \left[-\frac{\rho^{2}}{W^{2}(z)}\right] \exp \left[-j k z-j k \frac{\rho^{2}}{2 R(z)}+j \zeta(z)\right]
$$

Where $\mathrm{A}_{0}=\mathrm{A} /\left(\mathrm{jz} \mathrm{z}_{0}\right)$. The following beam parameters are as follows[4]:

$$
\begin{aligned}
& \mathrm{W}(\mathrm{z})=\mathrm{W}_{0}\left[1+\left(\frac{z}{z_{0}}\right)^{2}\right]^{1 / 2} \\
& \mathrm{R}(\mathrm{z})=\mathrm{z}\left[1+\left(\frac{z_{0}}{z}\right)^{2}\right] \\
& \zeta(\mathrm{z})=\tan ^{-1} \frac{z}{z_{0}} \\
& \mathrm{~W}_{0}=\left(\frac{\lambda \mathrm{z}_{0}}{\pi}\right)^{1 / 2}
\end{aligned}
$$

\subsubsection{Beam Intensity}

The optical intensity is a function of the distance $\mathrm{z}$ from the source and the radial distance $\rho=\left(x^{2}+y^{2}\right)^{1 / 2}$ of the beam plane[4].

$$
\mathrm{I}(\rho, \mathrm{z})=\mathrm{I}_{0}\left[\frac{W_{0}}{W(z)}\right]^{2} \exp \left[\frac{-2 \rho^{2}}{W^{2}(z)}\right]
$$

where $\mathrm{I}_{0}=\mid \mathrm{A}_{0 \mid}{ }^{2}$. The intensity along each plane of the $\mathrm{z}$ axis is a Gaussian curve having its peak at $\rho=0$ and decreasing radially in a monotonic fashion. The beam width $W(z)$ increases with increasing distance $z$. The beam has the following intensity at $\rho=0$ with its peak intensity at $\mathrm{z}=0[4]$. 


$$
\mathrm{I}(0, \mathrm{z})=\mathrm{I}_{0}\left[\frac{W_{0}}{W(z)}\right]^{2}=\frac{I_{0}}{\left(1+\left(\frac{z}{z_{0}}\right)^{2}\right)}
$$

The beam intensity reaches half its peak value at $\mathrm{z}= \pm \mathrm{Z}_{0}$.

\subsubsection{Beam Power}

The beam optical power is obtained by integrating over a transverse plane $z[4]$.

$$
\mathrm{P}=\int_{0}^{\infty} \mathrm{I}(\rho, \mathrm{z}) 2 \pi \rho \mathrm{d} \rho=\frac{1}{2} I_{0} \pi W_{0}^{2}
$$

The beam intensity can now be expressed as a function of power[4]

$$
\mathrm{I}(\rho, \mathrm{z})=\frac{2 \mathrm{P}}{\pi \mathrm{W}^{2}(z)} \exp \left[\frac{-2 \rho^{2}}{W^{2}(z)}\right]
$$

From this we can determine the ration of power contained in a circle of radius $\rho_{0}$ [4]

$$
\frac{1}{P} \int_{0}^{\rho_{0}} \mathrm{I}(\rho, \mathrm{z}) 2 \pi \rho \mathrm{d} \rho=1-\frac{-2 \rho^{2}}{W^{2}(z)}
$$

From this we find that a beam radius of $\rho_{0}=\mathrm{W}(\mathrm{z})$ contains approximately $86 \%$ of the total power while $99 \%$ of the beam power is contained in a radius of $1.5 \mathrm{~W}(\mathrm{z})$.

\subsubsection{Beam Radius, Divergence, and Depth of Focus}

The beam radius is defined as $\rho=W(z)$ where the beam intensity drops by a factor of $1 / \mathrm{e}^{2}$. The beam radius is determined by the following equation[4]. 


$$
\mathrm{W}(\mathrm{z})=\mathrm{W}_{0}\left[1+\left(\frac{z}{z_{0}}\right)^{2}\right]^{1 / 2}
$$

$\mathrm{W}_{0}$ is the waist radius and is the minimum beam radius value. The spot size is defined as $2 \mathrm{~W}(\mathrm{z})$ and increases along the $\mathrm{z}$ axis to a value of $\sqrt{ }(2) \mathrm{W}_{0}$ at the rayleigh range $\mathrm{z}_{0}$.

The beam diverges linearly far from the beam when $z>>z_{0}$. This defines a cone with half angle $\theta_{0}$ given by[4]:

$$
\theta_{0}=\frac{\lambda}{\pi \mathrm{W}_{0}}
$$

This shows that as the waist gets smaller and the wavelength gets larger, the beam diverges more. A highly directional beam can be obtained by having a large waist and small wavelength.

The Gaussian beam has its best focus at the position $\mathrm{z}=0$ and becomes more out of focus as the axial distance $\mathrm{z}$ grows larger. The depth of focus is defined as twice the rayleigh range, the point where the beam radius is a factor of $\sqrt{ }(2)$ of its minimum value. The depth of focus is defined as follows[4]:

$$
2 \mathrm{z}_{0}=\frac{2 \pi \mathrm{W}_{0}^{2}}{\lambda}
$$

From this we see that the depth of focus grows larger as the waist size increases and wavelength decreases. This demonstrates that we cannot have both a long depth of focus and small spot size. 


\subsubsection{Gas Lasers}

The following discussion is adapted from previous optics texts[8]. A LASER (Light Amplification by the Stimulated Emission of Radiation) is a process that produces a highly directional Gaussian beam with a narrow spectrum of wavelengths. Two ways this can be achieved is through the use of gas mixtures or semiconductor materials. Either means of producing laser light have the same underlying principle of producing coherent light amplification by creating a population inversion. This is the process of exciting electrons to a higher energy level then causing them to drop to a lower energy level by the introduction of photons with a wavelength that has energy equal to or greater than the band gap of the laser medium. This has the effect of causing an electron transition which emits a photon of light that is of the same wavelength and phase as that of the incident photons. This is achieved by an electrical discharge in a He-Ne laser when using a high DC or RF voltage. The ends of the laser have highly reflective mirrors which cause most of the light produced to be reflected back into the laser to help continue lasing. A small portion of the light is allowed to escape through an aperture at one end of the laser cavity. This output is a highly directional Gaussian beam which has all the properties mentioned previously in this chapter.

The frequency of light is determined by three main factors: excitation medium, the source used to produce stimulated emission, and the length of the laser cavity. The bandgap of the excitation material determines a range of frequencies possible from the laser since only photons with energy equal to or greater than the bandgap can produce stimulated emission. The emitted photons take on the same properties as the incident photons used to produce the stimulated emission. The length of the cavity determines the wavelengths that the laser can maintain. Only standing waves that have an integer multiple of half wavelengths which fit in the laser cavity length can be supported[8].

$$
m(\lambda / 2)=L
$$

The random motion of the atoms in a gas laser produce a spectrum of wavelengths through the Doppler effect. The gas atoms are in random motion with frequency $\mathrm{v}_{0}$. When the atom is moving way from the observer its frequency is seen as[8]: 


$$
\mathrm{v}_{1}=\mathrm{v}_{0}\left(1-\frac{v_{x}}{c}\right)
$$

When moving toward the observer its frequency is seen as [8]:

$$
\mathrm{v}_{2}=\mathrm{v}_{0}\left(1+\frac{v_{x}}{c}\right)
$$

This, coupled with the random motion of the atoms gives rise to Doppler broadened line width $\Delta \mathrm{v}=\left(\mathrm{v}_{2}-\mathrm{v}_{1}\right)$ of the output light from a gas laser. These wavelengths give a net envelope that is approximately Gaussian and usually form a narrow band of frequencies on the order of $\Delta \mathrm{v} \approx \lambda / 100$.

\subsection{Spectral Output}

It is important to know the frequency of the laser being used both to allow the power meter to measure the beam power and because the lenses were designed for use with specific wavelengths. The frequency value is required for input to the power meter for accurately testing the laser power. This is because the power meter measures the light intensity and uses a table to determine the power of the beam based on the frequency input by the user. The following spectral output was obtained by shining the laser into a spectrometer model SpectaPro500i from Acton Research. The following spectral output was obtained. ${ }^{1}$

\footnotetext{
${ }^{1}$ Spectral output measured by Chris Miller
} 


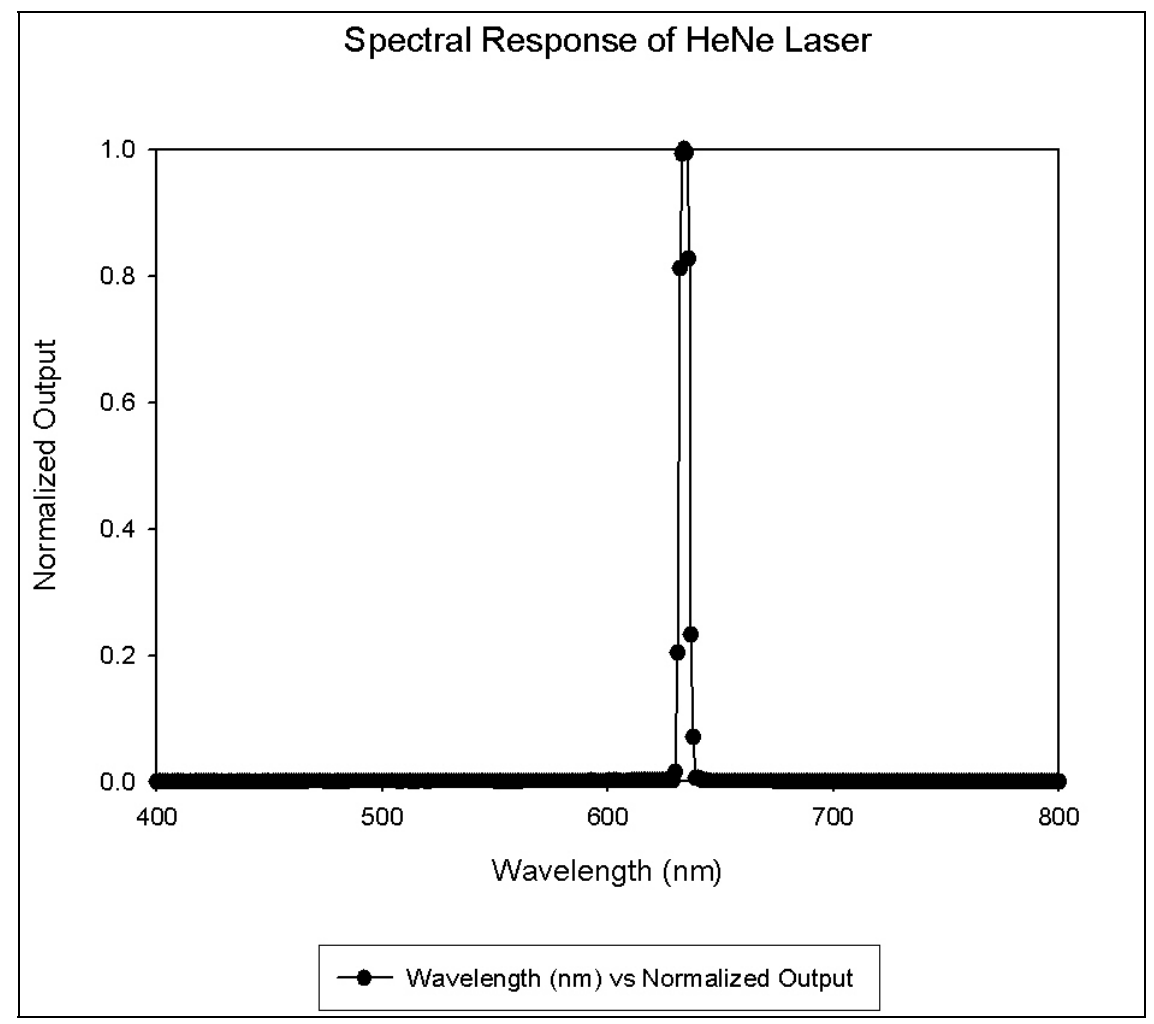

Figure 16 Spectral Response of HeNe Laser from 400-800nm

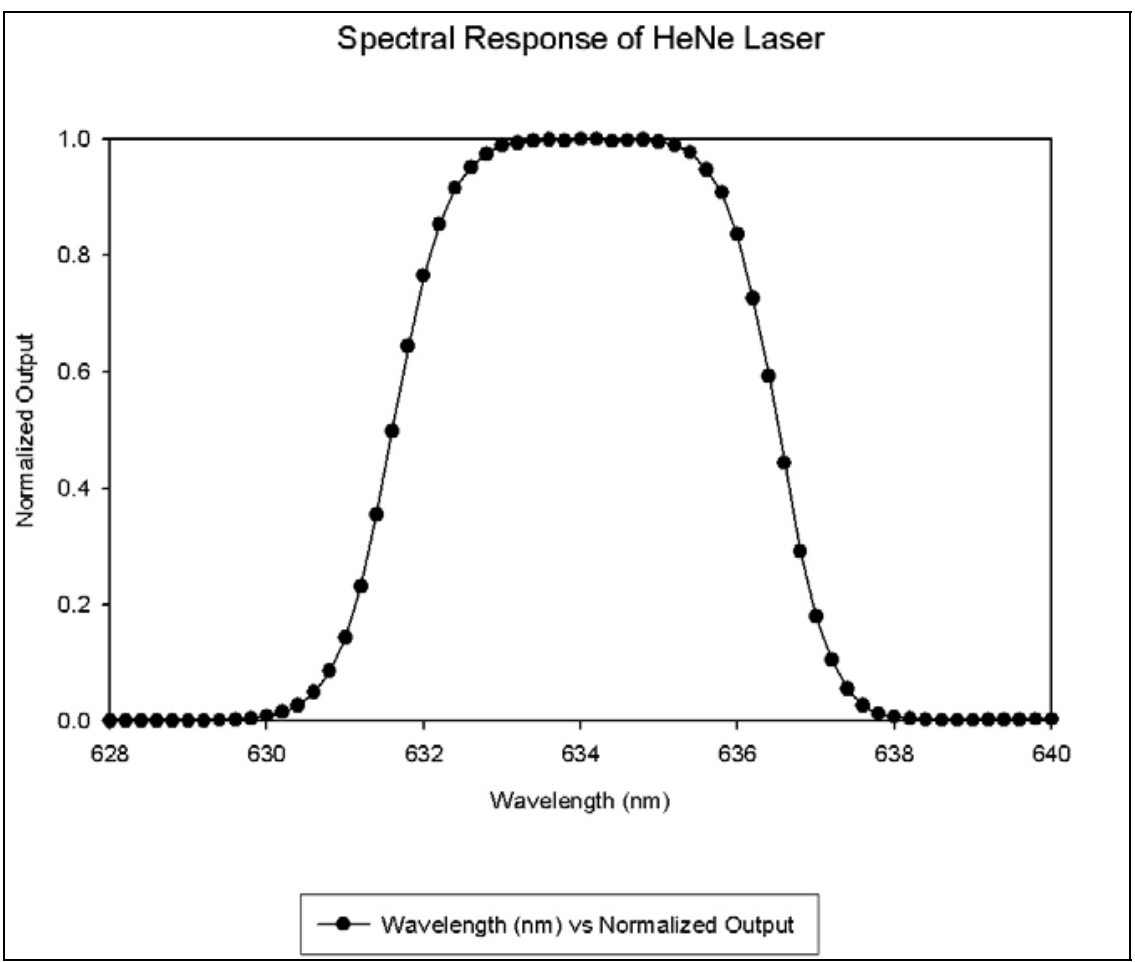

Figure 17 Spectral response of HeNe laser from 628-640nm 
The spectrum analyzer sampled over wavelengths from $400-800 \mathrm{~nm}$ and found the peak power output for the laser occurs between the wavelengths of $632.5-635.5 \mathrm{~nm}$. Each point represents one measurement.

It should be noted that using a power meter to measure the spectral response will not work. The power meter may be frequency selective but uses a lookup table and the sensors input to calculate the expected power at a given frequency. This number will not be accurate unless the frequency of the input light is known and that frequency is selected in the power meter.

\subsection{Waist Location, Waist Size, and Angular Divergence}

The waist location of a beam of light is the point where the diameter of the beam is its smallest. This waist location is in the beams focal plane. All points before and after this waist location diverge and the beam diameter gets larger.

The waist location, in this instance, is taken to be at the face of the laser enclosure since its output is collimated and a waist inside the enclosure cannot be verified experimentally. The angular divergence can still be measured with this assumption since it is a function of diameter change over distance.

The laser diameter was sampled with the CCD camera by placing the camera at various points away from the laser. The camera and laser were attached to an air cushioned workbench to minimize vibrations. The objects between the laser and camera in the following picture were not present when these measurements were taken. 


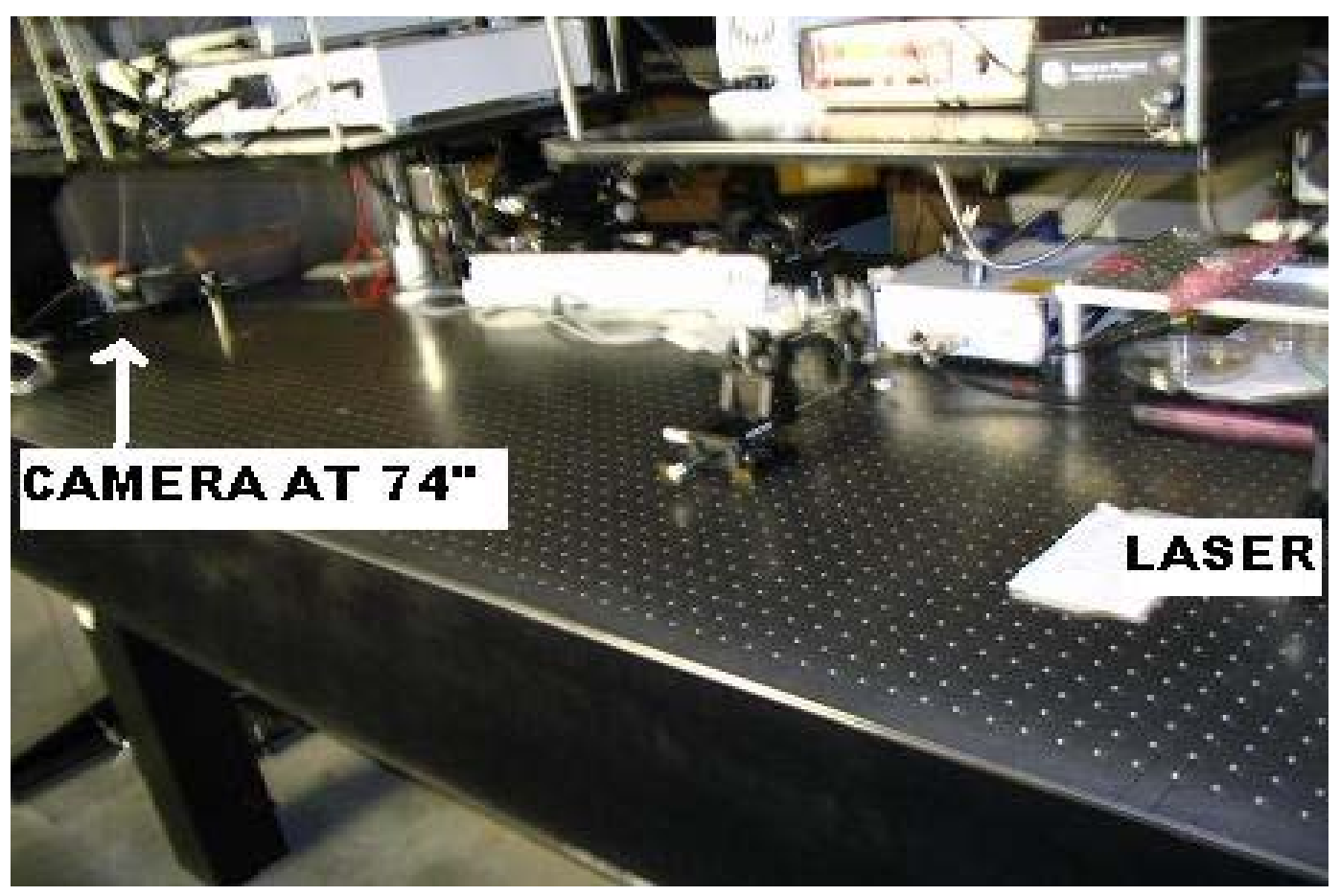

Figure 18 Workbench setup lab picture

The camera software gave the ability to measure the diameter using several different methods (4-sigma, FWHM, 13.5\% peak, etc.). The 4-sigma method measures the second moment beam diameter which is defined as four times the standard deviation of the beams energy distribution. The full width half maximum (FWHM) method measures the diameter at $50 \%$ of the beam peak. The software also allows measurement of the beam width at any percentage of the beam peak. The 4-sigma method was used to measure the diameter since this is taken as the industry standard for beam measurement and contains $95 \%$ of the beam energy distribution. It should be noted that for $\mathrm{TEM}_{00}$ (Gaussian beam) 2 -sigma is the $1 / \mathrm{e}^{2}$ diameter which contains $86 \%$ of the beam power.

Wave theory was used to predict what the laser divergence should be. The data collected was then overlaid onto the prediction curve with fairly accurate results.

Two separately collected sets of data were used to verify the accuracy of the beam diameter prediction curve. The first set of data was the preliminary trial while the second set of data was collected more carefully and over a longer distance from the camera. Each locations' diameter was measured by taking 125 samples from which the average 4-sigma diameter was 
determined. The Spiricon LBA-PC software used to collect the data performs this averaging operation.

The following wave theory equations were used to calculate the expected beam waist $\mathrm{W}_{0}$, rayleigh range $2 \mathrm{z}_{0}$, and angular divergence $\theta_{0}[4]$ :

$$
\begin{aligned}
& \mathrm{W}(\mathrm{z})=\mathrm{W}_{0}\left[1+\left(\mathrm{z} / \mathrm{z}_{0}\right)^{2}\right]^{1 / 2} \\
& 2 \mathrm{z}_{0}=2 \pi \mathrm{W}_{0}^{2} / \lambda \\
& \theta_{0}=2 \lambda /\left(\pi 2 \mathrm{~W}_{0}\right)
\end{aligned}
$$

$\mathrm{W}(\mathrm{z})$ is the radius of the beam at distance $\mathrm{z}$ from the laser, and $\lambda$ is the wavelength of the light. The value $\mathrm{z}_{0}$ shows the FWHM point on the Gaussian intensity profile of the beam. Solving (23) for $\mathrm{W}_{0}$ gives:

$$
\mathrm{W}_{0}=\left(\mathrm{z}_{0} \lambda / \pi\right)^{1 / 2}
$$

Substituting (25) into (22) and solving for $\mathrm{z}_{0}$ gives:

$$
\mathrm{z}_{0}=\left[\mathrm{W}^{2}(\mathrm{z}) \pi \pm \sqrt{ }\left(\mathrm{W}^{4}(\mathrm{z}) \pi^{2}-4 \lambda^{2} \mathrm{z}^{2}\right)\right] / 2 \lambda
$$

Using the plus sign in the numerator gives the correct prediction results for the beam radius. All quantities in equation (26) are known except $\mathrm{W}(\mathrm{z})$ and $\mathrm{z}_{0}$. A diameter value $(2 \mathrm{~W}(\mathrm{z}))$ of the beam was measured at a distance $\mathrm{z}$ and substituted into the above equations. A prediction curve for the beam diameter was formed from this.

A beam diameter value $\mathrm{W}(\mathrm{z})$ was then measured at a distance of $0.30 \mathrm{~m}$ from the laser and used to calculate the prediction curve in Figure 19. Any of a number of beam radius measurements could be used to make the prediction curve. The 0.30 meter mark was chosen because that value produced accurate divergence prediction results and the beam at that point 
was a uniform Gaussian shape. It will be shown later that beam diameters measured closer than 0.30 meters to the laser produce non-uniform Gaussian profiles.

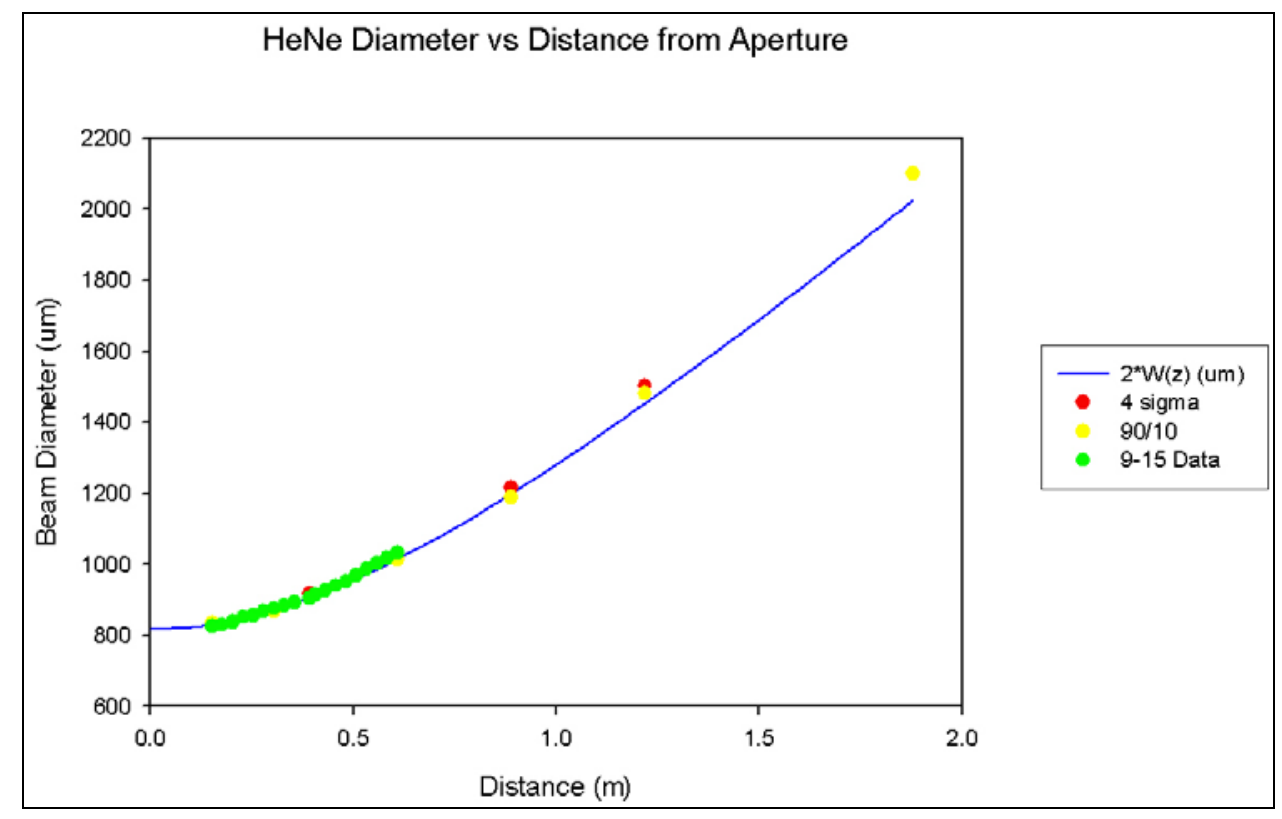

Figure 19 HeNe beam diameter prediction curve and data

The blue line is the predicted laser diameter, green is the first set of collected data, and the yellow and red are diameters from the second set of collected data. Each point represents an average diameter calculated from 125 samples. The standard deviation of the diameter for each point ranges from $0-5 \mathrm{um}$ and so the error bars are not visible on this graph. The zero point on the $\mathrm{x}$-axis signifies the face of the laser housing and 1.778 meters is the furthest point from the laser that the beam can be measured on the workbench.

The following graph represents the error over distance from the laser housing between the first set of data and the prediction curve generated using the 0.3048 meter waist radius $\mathrm{W}(\mathrm{z})$. Each point represents the difference between the average 4-sigma beam diameter (each measurement having 125 samples) and the prediction curve. The error bars are taken from the standard deviation of the beam diameter measurements. 


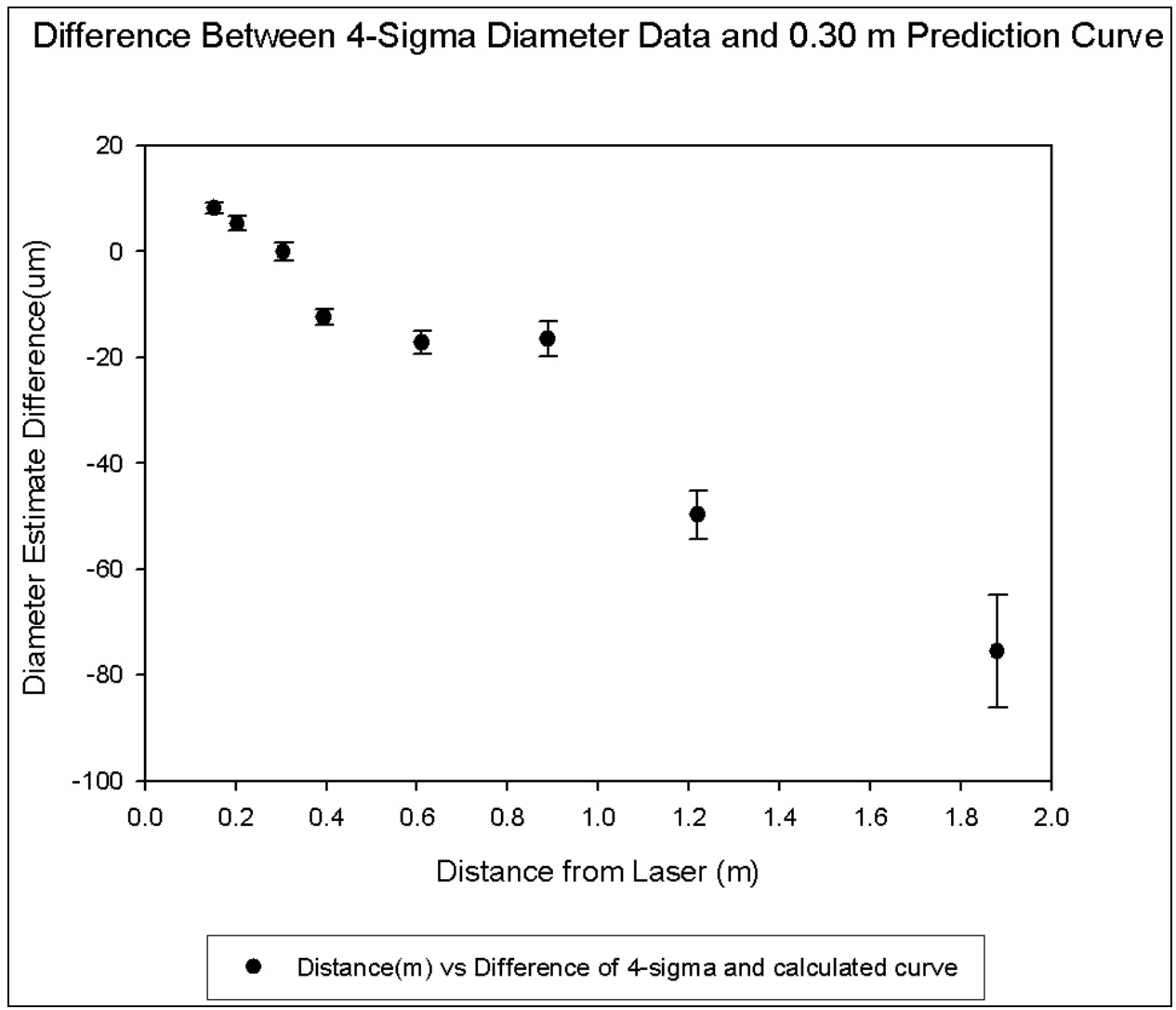

Figure 20 Difference between 4-Sigma beam diameter measurements and prediction curve

The negative values show where the prediction curve underestimates the value of the beam diameter.

Various $\mathrm{z}$ distances and their associated beam radius values $\mathrm{W}(\mathrm{z})$ could be used in (26) to predict the divergence of the laser. Figure 21 shows that using beam radius values corresponding to larger distances from the laser caused increasing inaccuracies in the divergence prediction curve. 


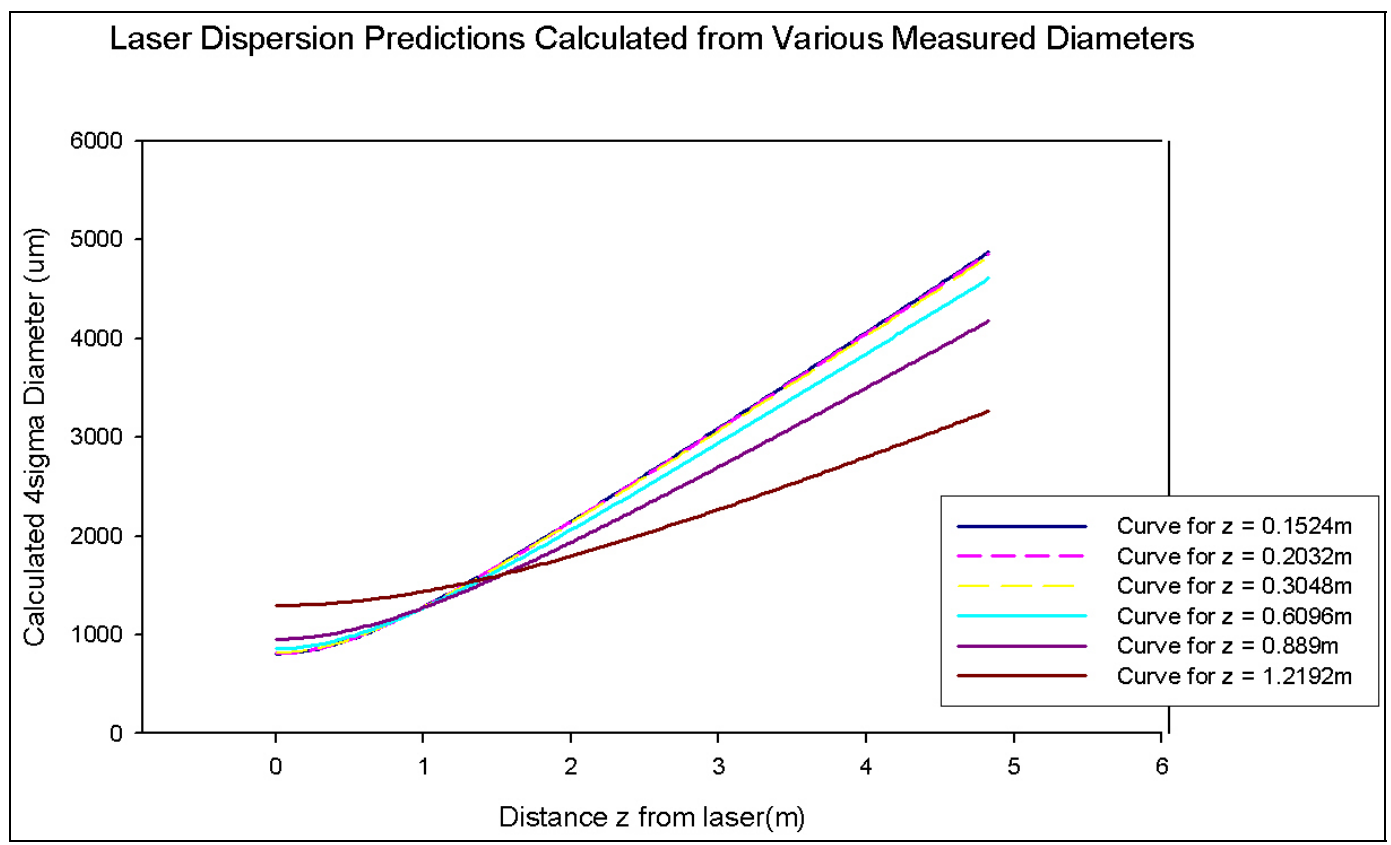

Figure 21 Various HeNe prediction curves

Beam radius values taken at $0.15,0.20$, and 0.30 meters from the laser produce fairly accurate prediction curves. Beam radius values at distances of 0.61 meters and beyond used to predict $\mathrm{W}(\mathrm{z})$ seem to produce inaccurate results. All of the curves should be the same at any distance from the laser since they all predict the same laser diameter $\mathrm{W}(\mathrm{z})$. The distances further from the laser may suffer from inaccurate beam diameter measurements due to some vibrations. If these vibrations are the cause, they would get worse at increasing distances from the laser. 


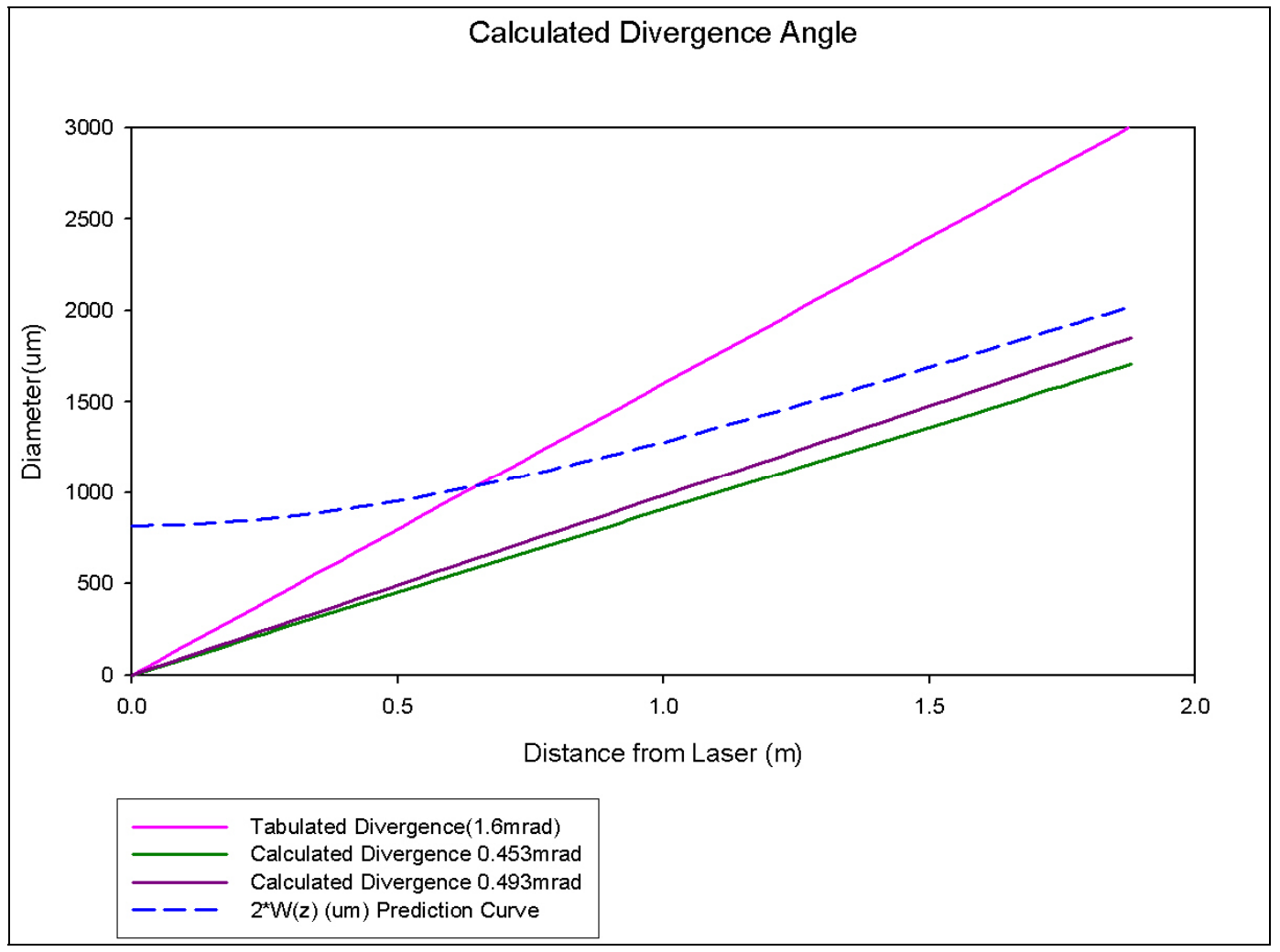

Figure 22 Divergence angle of HeNe laser

Figure 22 displays the previously calculated blue prediction curve using the beam diameter measured at 0.30 meters from the laser. The purple line is the calculated divergence angle of $0.4926 \mathrm{mrad}$ corresponding to diameter $2 \mathrm{~W}(\mathrm{z})$ from the prediction curve. The green line is a $0.4538 \mathrm{mrad}$ divergence found experimentally, while the pink line shows the data sheet tabulated divergence of the laser. I believe this tabulated divergence to be incorrect because the data sheet used may not be specifically for this laser. The manufacturer cross referenced this laser model to the data sheet he provided. The divergence should not decrease over time.

The divergence angle found experimentally using the first set of data, while not as carefully collected, does correspond closely to the angle calculated from the prediction curve which used the second set of collected data. This leads toward the conclusion that the prediction curve is fairly accurate over s distance of 1.89 meters. 


\subsection{Pointing Stability}

Attempts have been made to determine the pointing stability of the laser. The Spiricon software has a program that will do this. The program produces a scatter plot of the peak laser power outputs over which a centroid is calculated. This centroid has major and minor axis values that are two standard deviations from the average peak location. The workbench was air cushioned to limit outside vibrations so most variations should come from the laser. Theses results may explain why the diameter prediction curve is off for $\mathrm{W}(\mathrm{z})$ at farther distances from the laser. The diameter of calculated centroid should get larger for distances farther from the laser which would cause the laser diameter to vary more at longer distances. This variation would cause the prediction curve to be more inaccurate when using waist diameters $\mathrm{W}(\mathrm{z})$ that are farther from the laser.

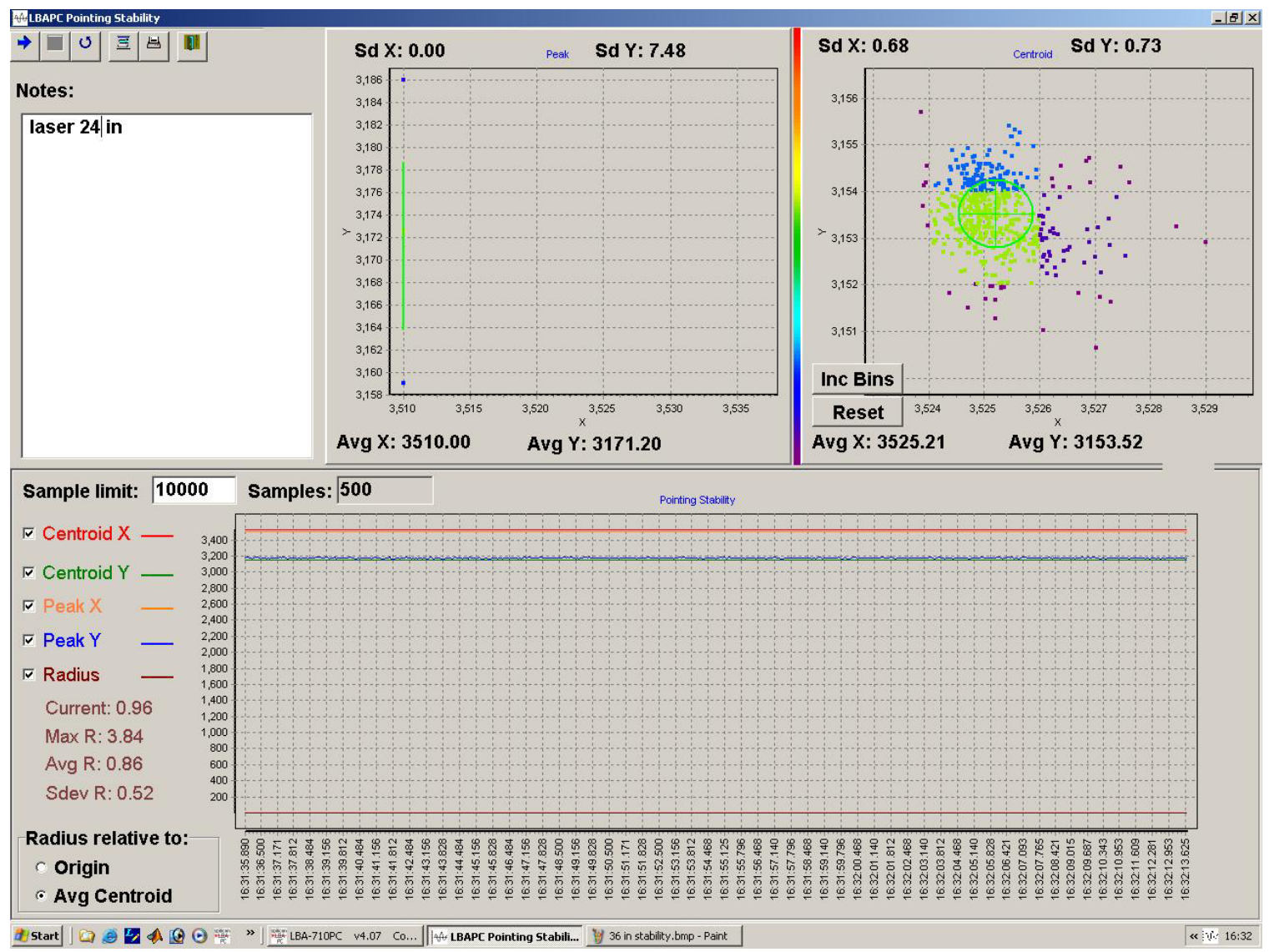

Figure 23 Spiricon pointing stability screen 
Figure 23 is an example of laser beam peak values measured at 0.61 meters from the laser using the Spiricon program. Each point represents one peak beam sample. The bottom graph of Figure 23 shows each beam peak point collected for a total of 125 points. The smoothness of the lines indicate a relatively stable beam. The beam maximum will, on average, fall within a radius of $0.86 \pm 0.52 \mathrm{um}$ for the measurements shown in Figure 23.

Figure 24 shows an average diameter over which the beam peak intensity can be found at various distances. Each point represents an average diameter calculated from 125 samples. Error bars represent the standard deviation as calculated in the pointing stability program. There is a trend toward larger instability at further distances from the laser. This could be due to outside vibrations, the laser itself, or other unknown factors.

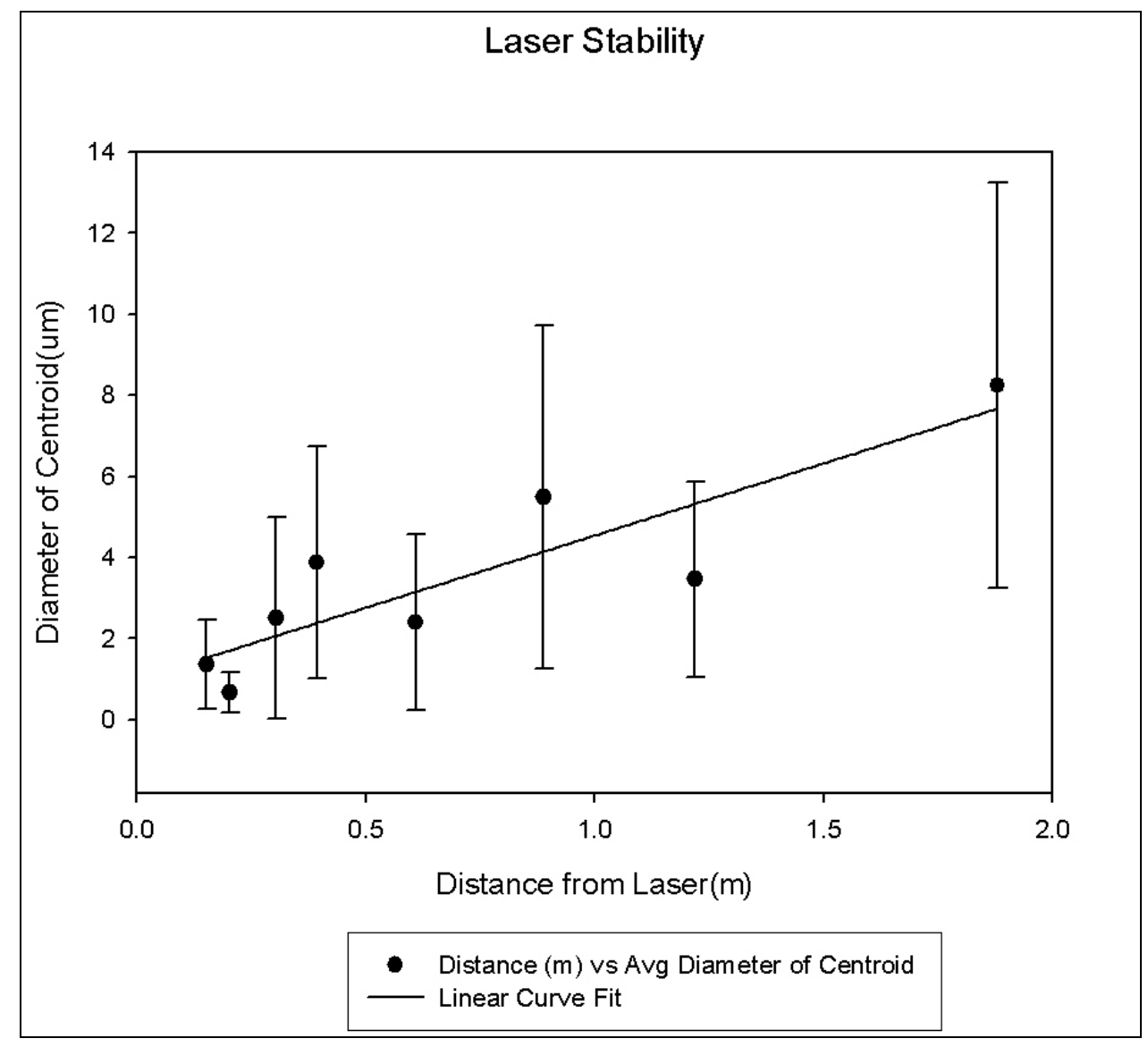

Figure 24 Laser pointing stability graph 
Any consistent vibration instability observed should produce a linearly increasing centroid size as the distance from the laser increases due to the linear angular dispersion of the beam at long distances. There may be other contributing factors since this linearity is not observed.

\subsection{Power Dissipation Over Distance}

The power of the beam maintains a Gaussian shape but power does get redistributed as the diameter of the beam increases. The measured beam diameter is roughly $2 \mathrm{~mm}$ at a distance of 1.89 meters from the laser. This is well within the $10 \mathrm{~mm}$ diameter of the PM100 power meter used and ensures that all the laser power is incident on the meter sensor and not lost when taking power measurements.

The power was measured by fixing the sensor head to a skid that moved along a track which was fixed to the workbench. This allowed the sensor to be moved in a $\mathrm{z}$-direction away from the laser while maintaining the same $x-y$ and angular orientation relative to the laser. The sensor was angled slightly so the laser light was not reflected back into the laser. This is done so that reflections from the sensor do not feed back into the laser and give a false power reading.

The power readings observed seem to be slightly dependent on the orientation of the sensor relative to the incident wave. Continuous readings were taken over the course of one minute at each location and found to be roughly $6.9-7 \mathrm{~mW}$ at all points from the laser. 


\begin{tabular}{|c|c|c|c|c|c|}
\hline \multirow{2}{*}{$\begin{array}{l}\text { Distance } \\
\text { From } \\
\text { Laser (in) }\end{array}$} & \multicolumn{5}{|l|}{ Laser power } \\
\hline & Current(W) & $\operatorname{Min}(W)$ & $\operatorname{Max}(W)$ & $\operatorname{Avg}(W)$ & Std.(W) \\
\hline 6[ & $\begin{array}{l}7.020 \mathrm{E}-03 \\
\end{array}$ & 6.999E-03 & $\begin{array}{l}7.021 \mathrm{E}-03 \\
\end{array}$ & 7.014E-03 & 9.162E-06 \\
\hline 8 & 7.044E-03 & 7.026E-03 & 7.047E-03 & 7.040E-03 & 7.751E-06 \\
\hline 12 & 7.008E-03 & 6.992E-03 & 7.009E-03 & 7.004E-03 & $6.189 \mathrm{E}-06$ \\
\hline 14 & & & & & \\
\hline 16 & & & & & \\
\hline 18 & $6.989 \mathrm{E}-03$ & $6.973 \mathrm{E}-03$ & $6.992 \mathrm{E}-03$ & 6.986E-03 & $5.416 \mathrm{E}-06$ \\
\hline 20 & 6.901E-03 & 6.884E-03 & 6.901E-03 & 6.894E-03 & $3.249 \mathrm{E}-06$ \\
\hline 22 & & & & & \\
\hline 24 & $6.931 \mathrm{E}-03$ & $6.930 \mathrm{E}-03$ & $6.950 \mathrm{E}-03$ & $6.941 \mathrm{E}-03$ & $0.000 \mathrm{E}+00$ \\
\hline 35 & & & & & \\
\hline 48 & & & & & \\
\hline
\end{tabular}

Table 9 Laser power measurements

Background power was measured at this wavelength and found to be on the order of $10^{-9} \mathrm{~W}$ and so had negligible contribution to the readings.

\subsection{In-Plane Power Distribution and Beam Shape}

The laser beam takes on a Gaussian form. This is seen in the Spiricon program when sampling the beam.

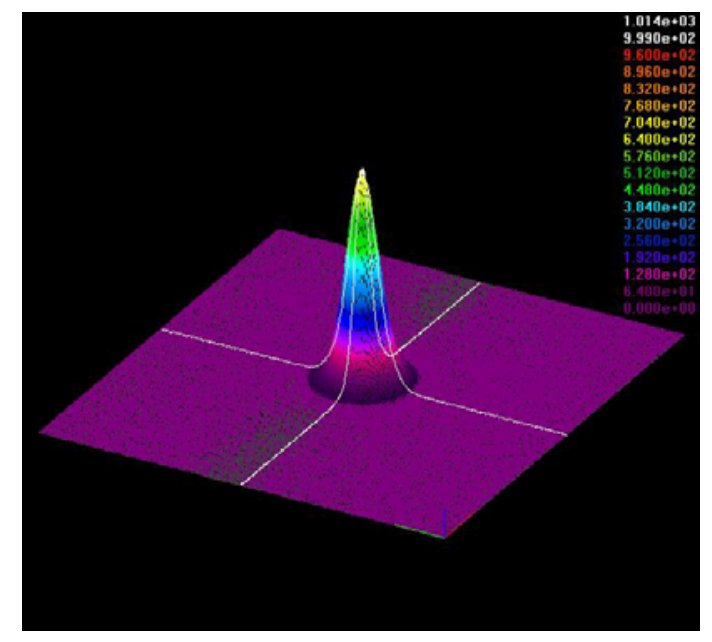

Figure 25 HeNe laser beam sampled profile in Spiricon

The items of interest here are the beam shape and the distribution of power. Measurements of the beam shape taken at 0.15 and 0.20 meters from the laser showed unexpected non- 
Gaussian results. Multiple time lapse measurements of 125 samples each indicate that the results do not fluctuate over time.
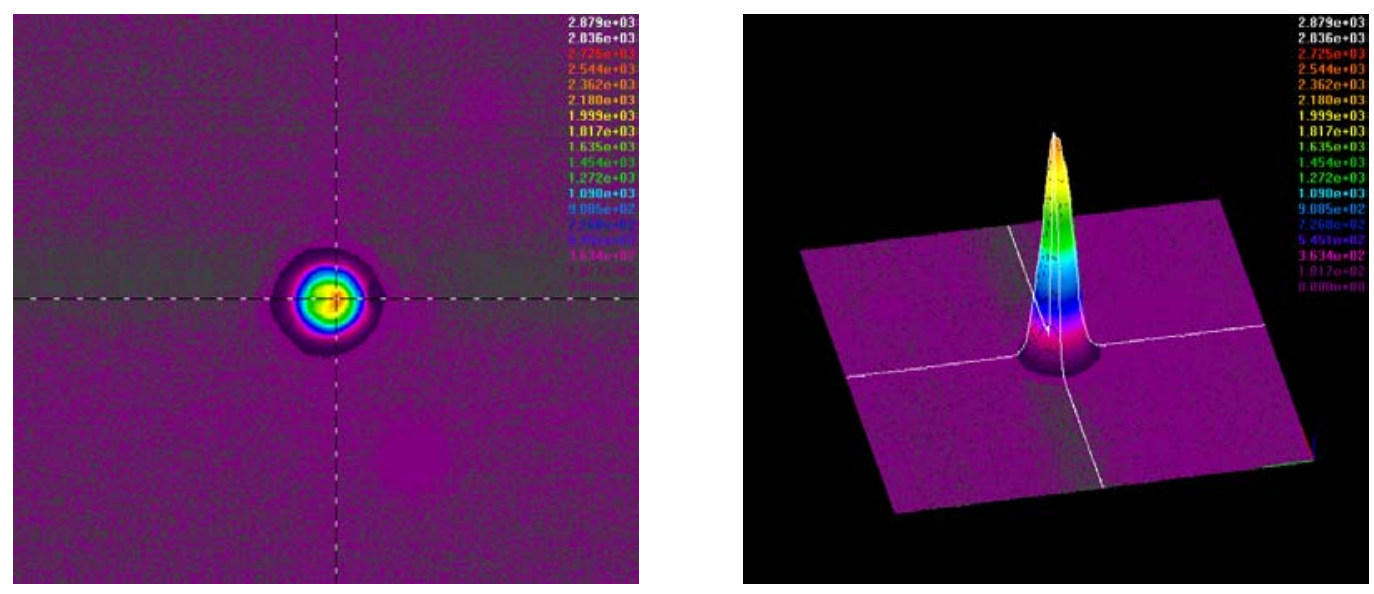

Figure 26 2D and 3D picture of HeNe beam at 0.15 meters in Spiricon
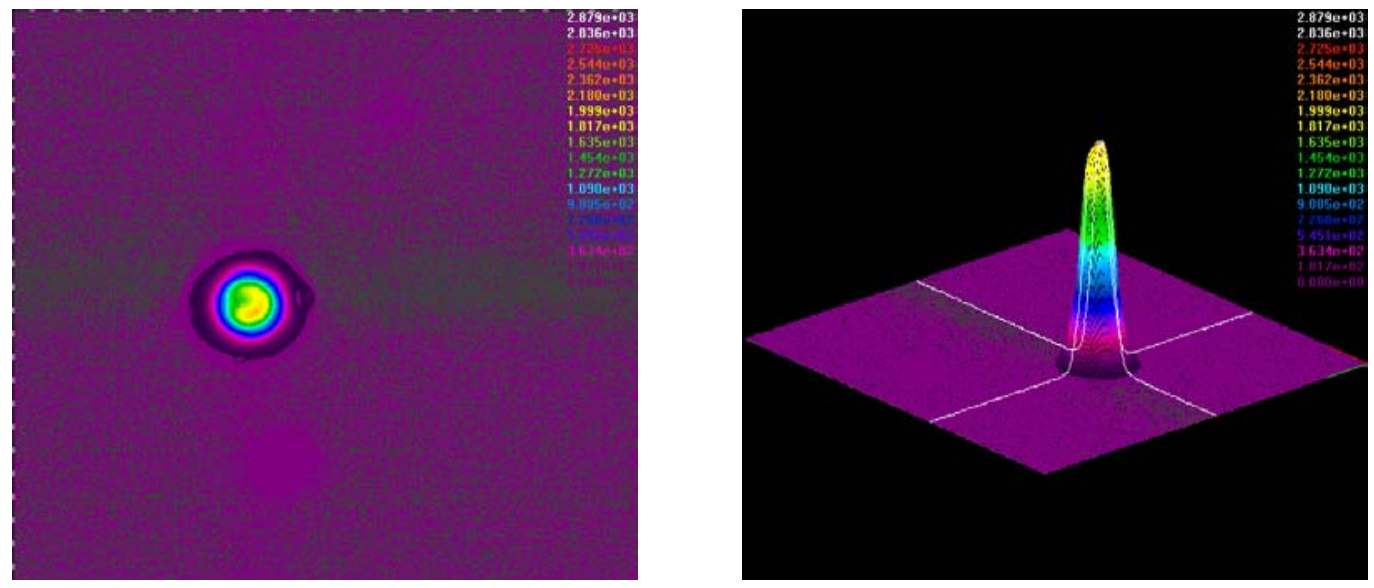

Figure 27 2D and 3D picture of HeNe beam at 0.20 meters in Spiricon

Figure 26 shows the non-Gaussian behavior demonstrated by the laser at $0.15 \mathrm{~m}$. Figure 27 shows the same behavior at $0.20 \mathrm{~m}$. The beam becomes Gaussian in shape by 0.30 meters from the laser and maintains this profile through a distance of at least 1,88 meters from the laser housing. The cause of the non-Gaussian beam profile near the laser case is unknown. 
The next item to consider is the power density profile along an arbitrary plane normal to the laser. This is done at various points away from the laser to investigate power density change as the beam propagates away from the laser.

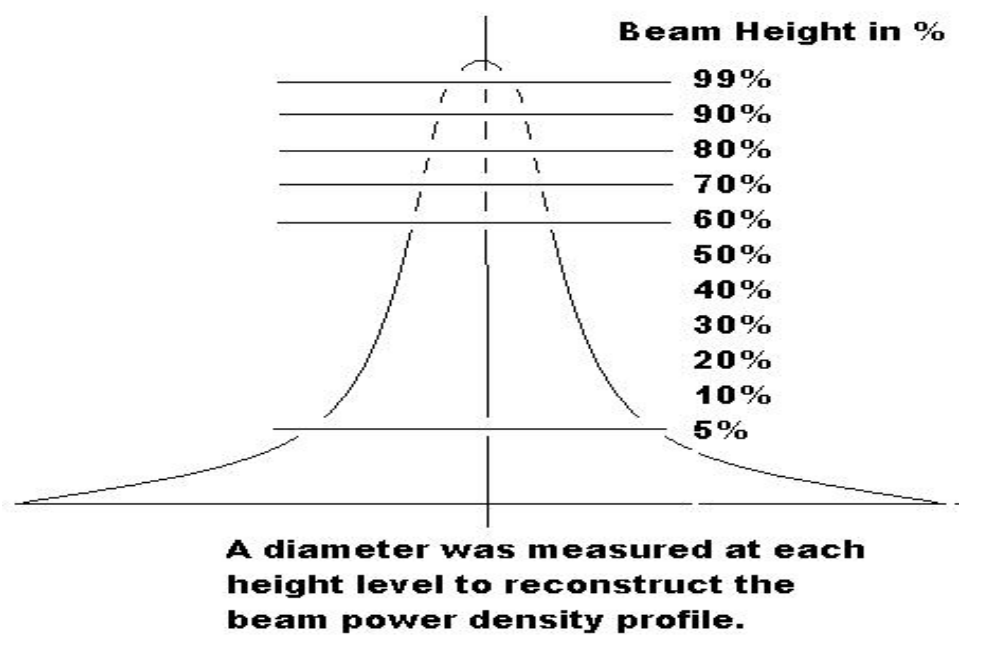

Figure 28 Drawing explaining how power profile is found

Figure 28 shows the power density profile of the laser in an arbitrary plane normal to the propagation direction. Each level corresponds to a percentage of the peak beam power density. At $100 \%$, the peak power density level of the beam is reached. At $0 \%$, the power density is zero. A diameter measurement is taken at various $\mathrm{z}$ locations from the laser and the peak power density of the beam is measured at that location. The power density profile at that location is then constructed in the Spiricon program by taking various percent peak levels of the intensity profile and multiplying them by the peak power density.

The power of the laser was measured previously to be $\sim 6.9 \mathrm{~mW}$. This value was put into the Spiricon software so it could calculate the power density at the peak. The following graph shows the beam power density versus radius. This was done at various distances from the laser. Each point represents an average radius measurement taken over 125 samples. 


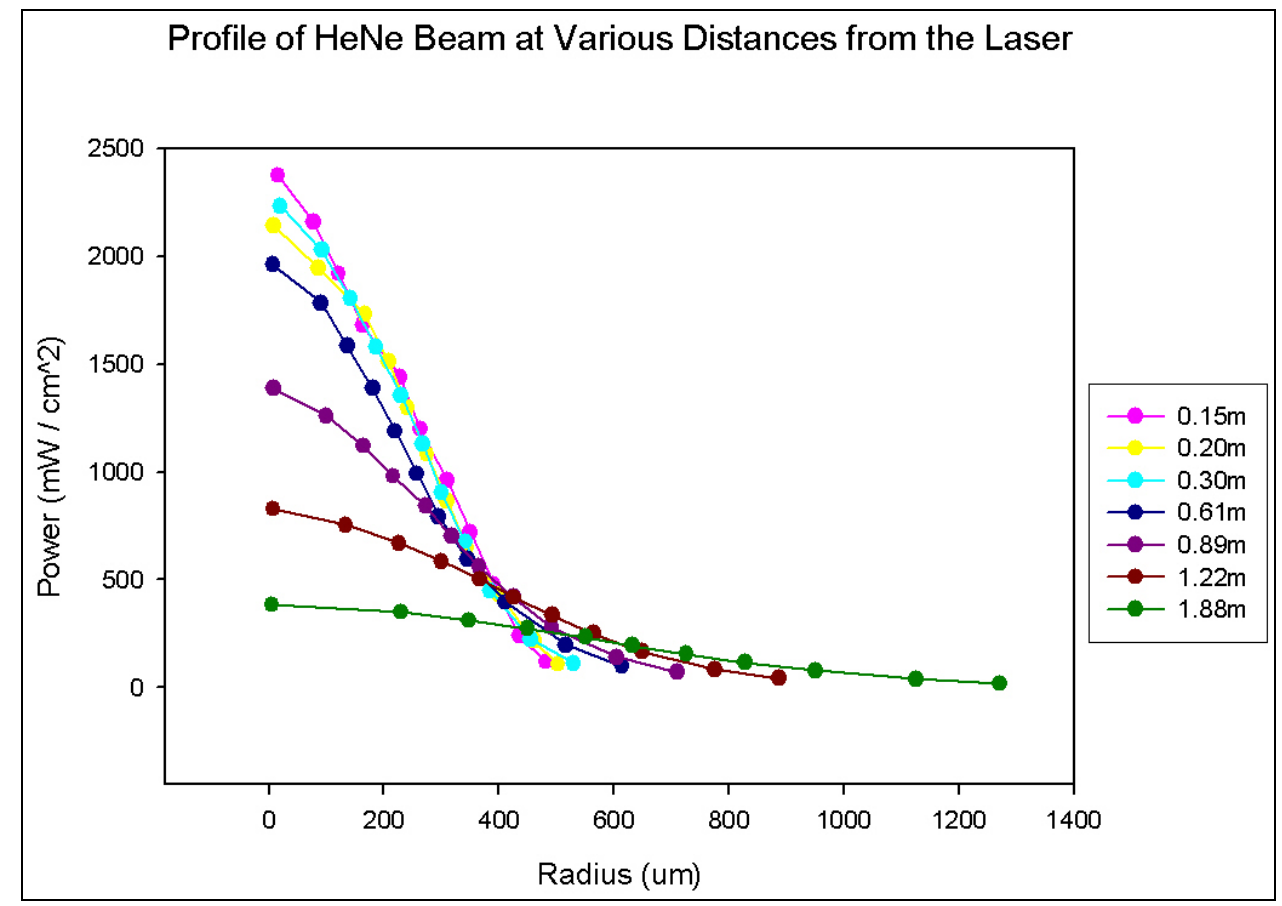

Figure 29 Power density profile of HeNe beam at various distances from laser housing

Figure 29 shows the peak power density diminishing as the beam travels from the laser and its diameter increases. That is expected since the power of the beam gets dispersed across a larger beam radius as the beam propagates. The error bars for each point range from 1.6-6.2 $\mathrm{mW} / \mathrm{cm}^{2}$ and are not visible on the graph. This graph does not fully capture any nonGaussian properties of the beam because it only uses a few radius values. Even so, the curves of the 0.15 and 0.20 meter distances have noticeable kinks indicating that more measurements might reveal the non-uniformity at these distances. This may also be indicated by the lower power density of the 0.20 meter beam relative to the 0.30 meter beam. The non-Gaussian shape at the 0.20 meter distance from the laser housing causes the peak power density to be lower than it normally would be. A 3-dimensional graph displaying the diameter and showing more points might capture the non-Gaussian profile better. The best method is to use the Spiricon software to do this for us.

The graph in Figure 30 shows the drop in power density of the laser as it propagates. The standard deviation ranges from 1-6 $\mathrm{mW} / \mathrm{cm}^{2}$ and cannot be seen on this graph. The drop from 0.15 to 1.89 meters is $2059 \mathrm{~mW} / \mathrm{cm} 2$, an $84 \%$ drop in peak power density. Each point represents the power density calculated over 125 samples at each location. 


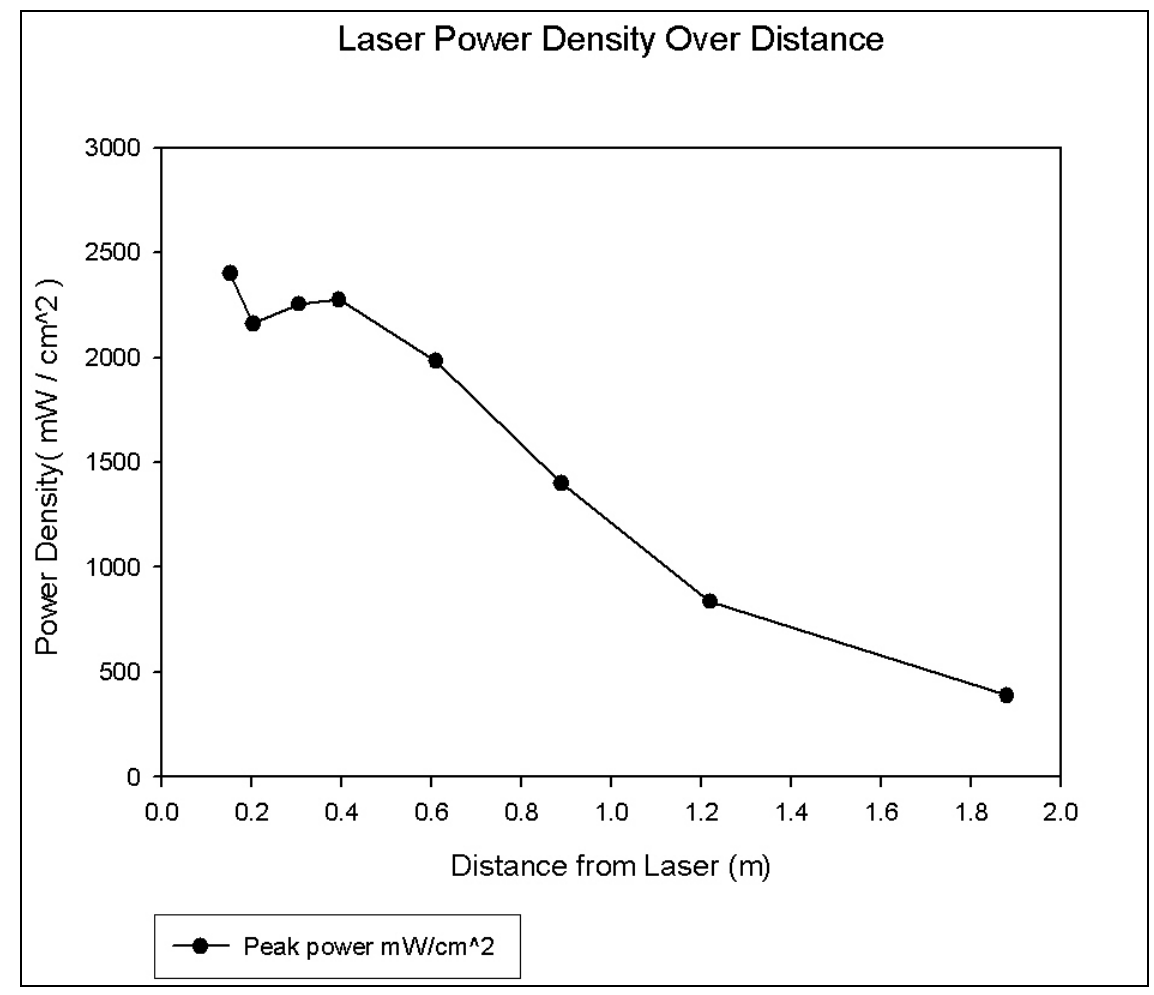

Figure 30 Power density over distance from laser

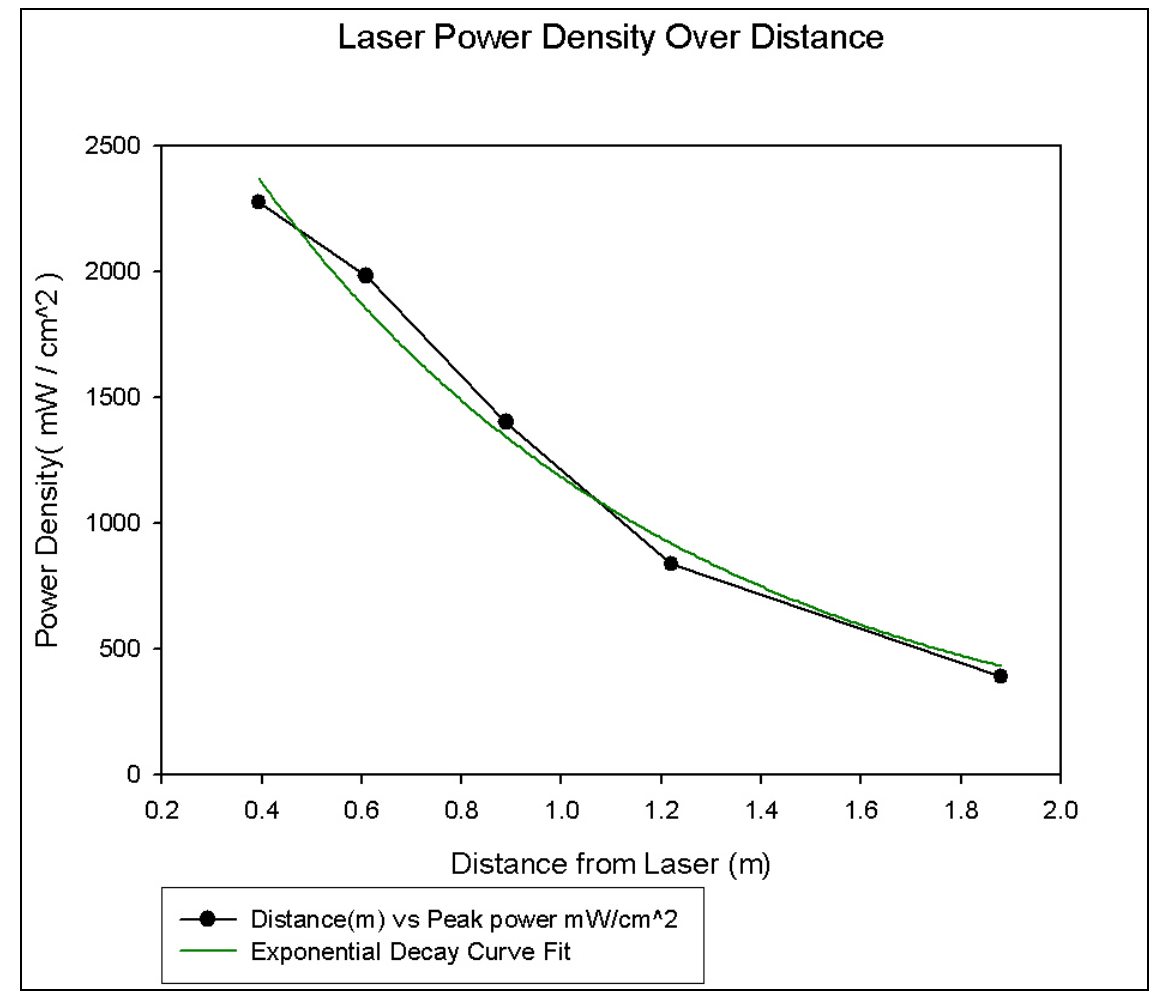

Figure 31 Power density curve fit 
The power density plot is closely resembled with a decreasing exponential curve after the non-Gaussian points closer than 0.30 meters to the laser are removed. This curve starts at 0.39 meters from the laser. 


\section{Chapter 4}

\section{Borofloat Characterization}

The lenses were etched into a borofloat wafer which requires the characterization of the effects of the wafer on the incident laser light. Tests in this area deal with the power loss of the borofloat which has an impact on lens efficiency.

The transmission of the borofloat was measured to be approximately $92 \pm 0.0609 \%$. This agrees with the graph from the manufacturer mentioned in previous work[1]. A portion of the borfloat near the bottom of the wafer was chosen for the measurements because this region had nothing etched into the wafer. That portion of the wafer was cleaned with a lens cloth and dust was blown off with compressed air. The extreme outer edge ( 1-2mm) of the wafer was avoided since this area was very cloudy. The laser, power sensor, and borofloat wafer were fixed to the bench using clamps. The laser and power meter sensor were then aligned making sure not to point the reflected light back into the laser aperture. The wafer was made to be perpendicular to the beam by pointing the reflected beam from the wafer near but not into the laser aperture. Twenty average power readings (including standard deviation) were taken of the light that was transmitted through the borofloat over an area of approximately $13 \mathrm{~mm}$ by $2 \mathrm{~mm}$. Each power reading was taken over approximately one minute. All twenty readings were averaged together to obtain $92 \%$ transmission through the borfloat. Reading number two was thrown out since it gave an efficiency of greater than $100 \%$. This was likely due to some extra reflected light entering the power meter. 


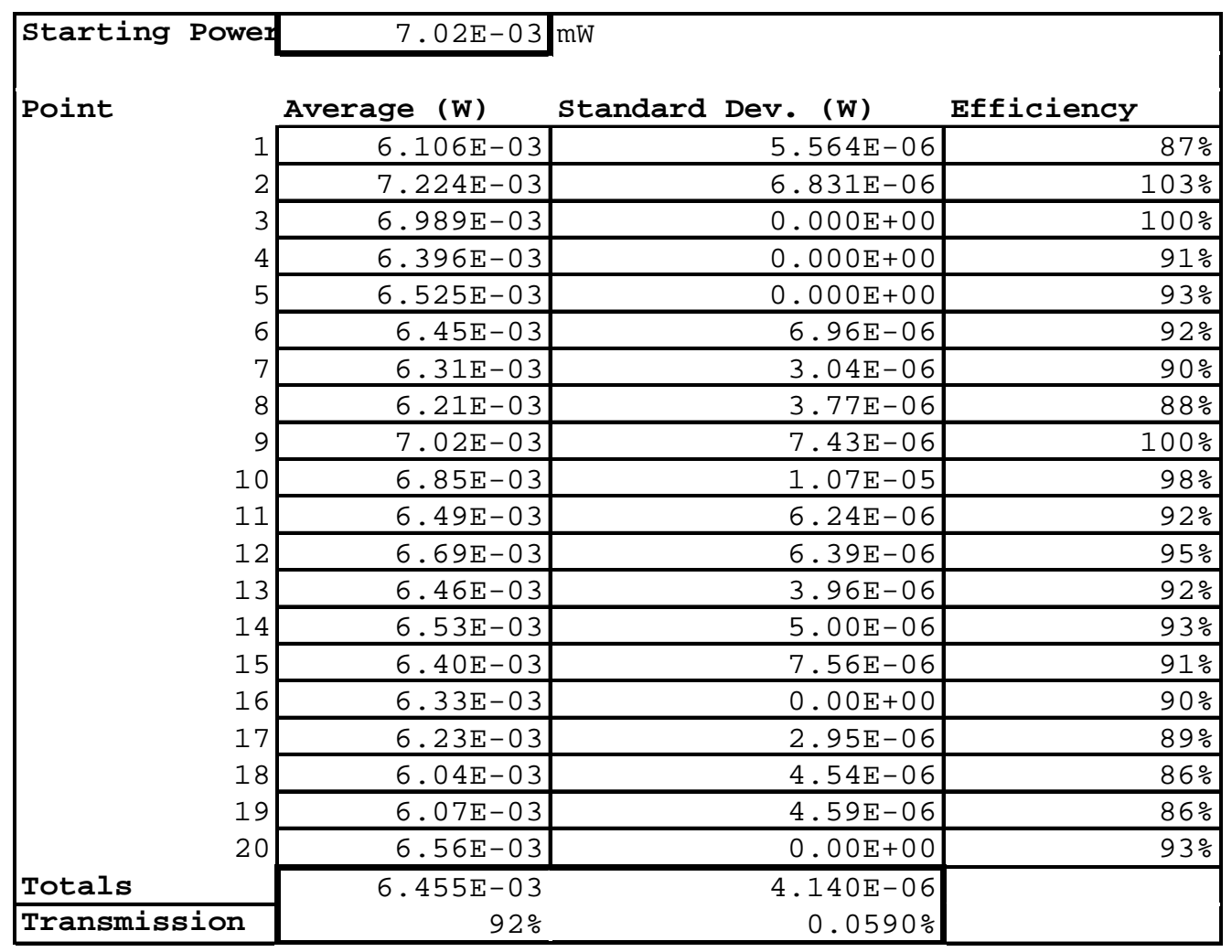

Table 10 Data collected for Borofloat Transmission.

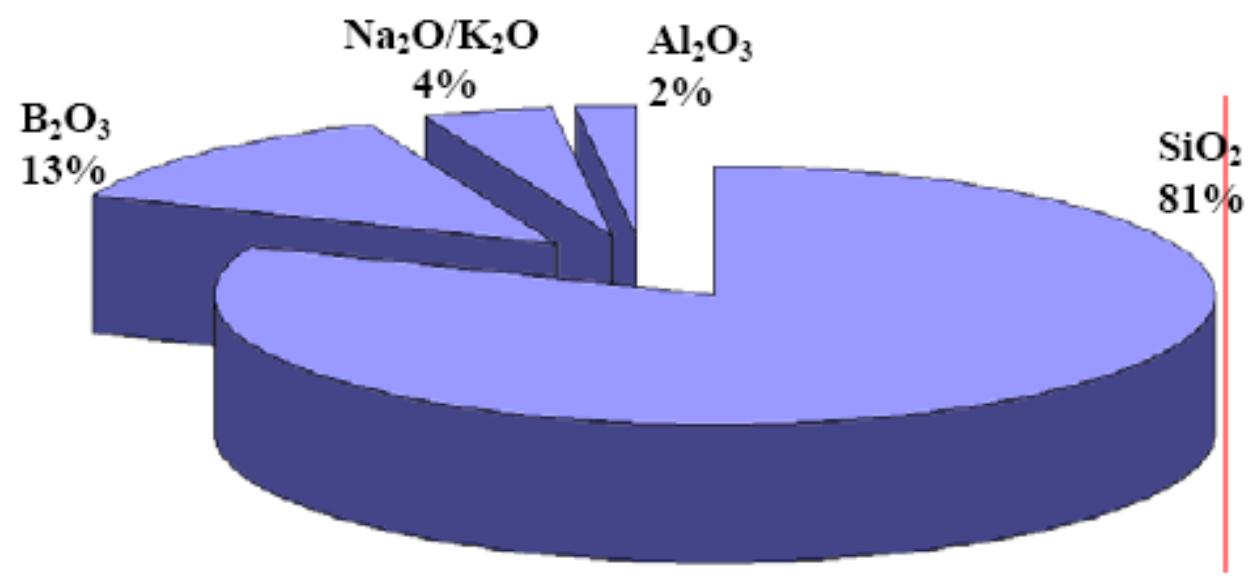

Figure 32 Breakdown of Borofloat material composition[15] 


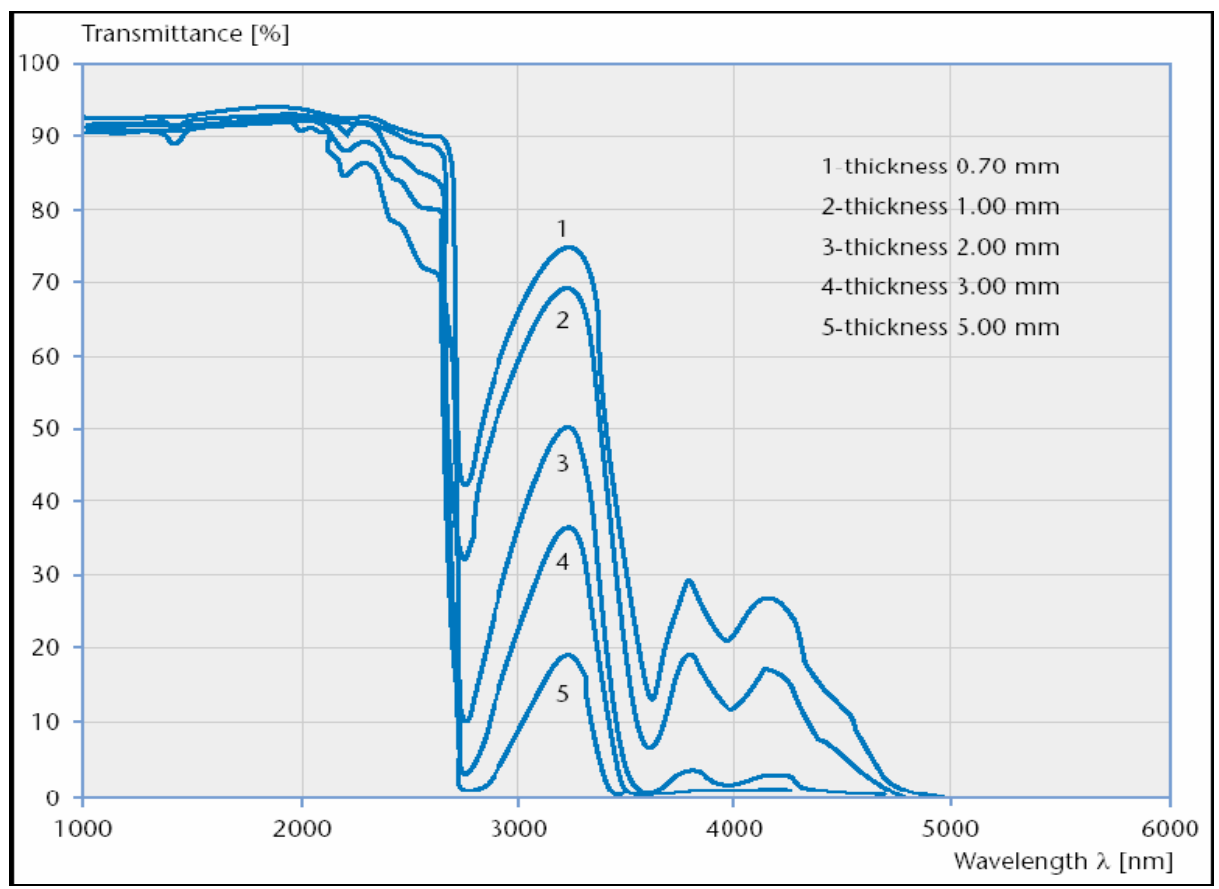

Figure 33 Borofloat tranmittance vs. IR wavelength [17]

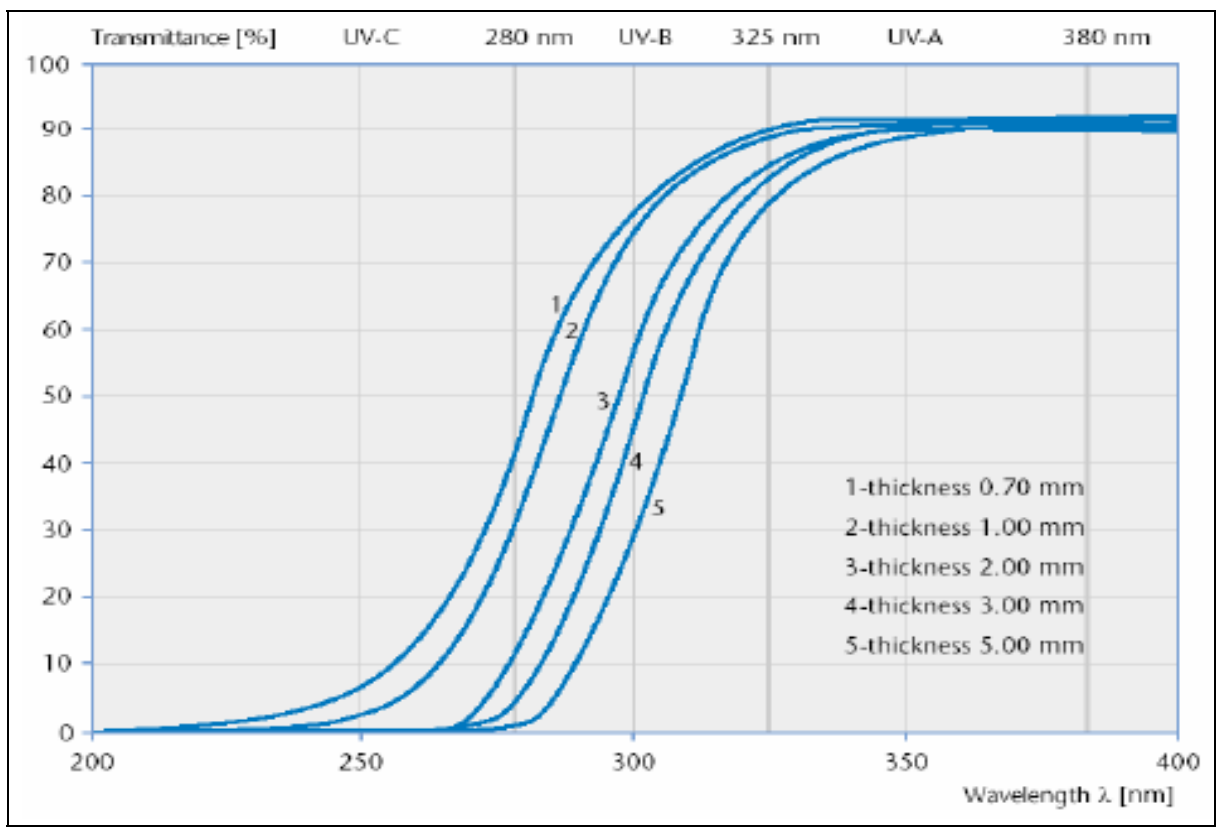

Figure 34 Borofloat transmittance vs. UV wavelength [16] 


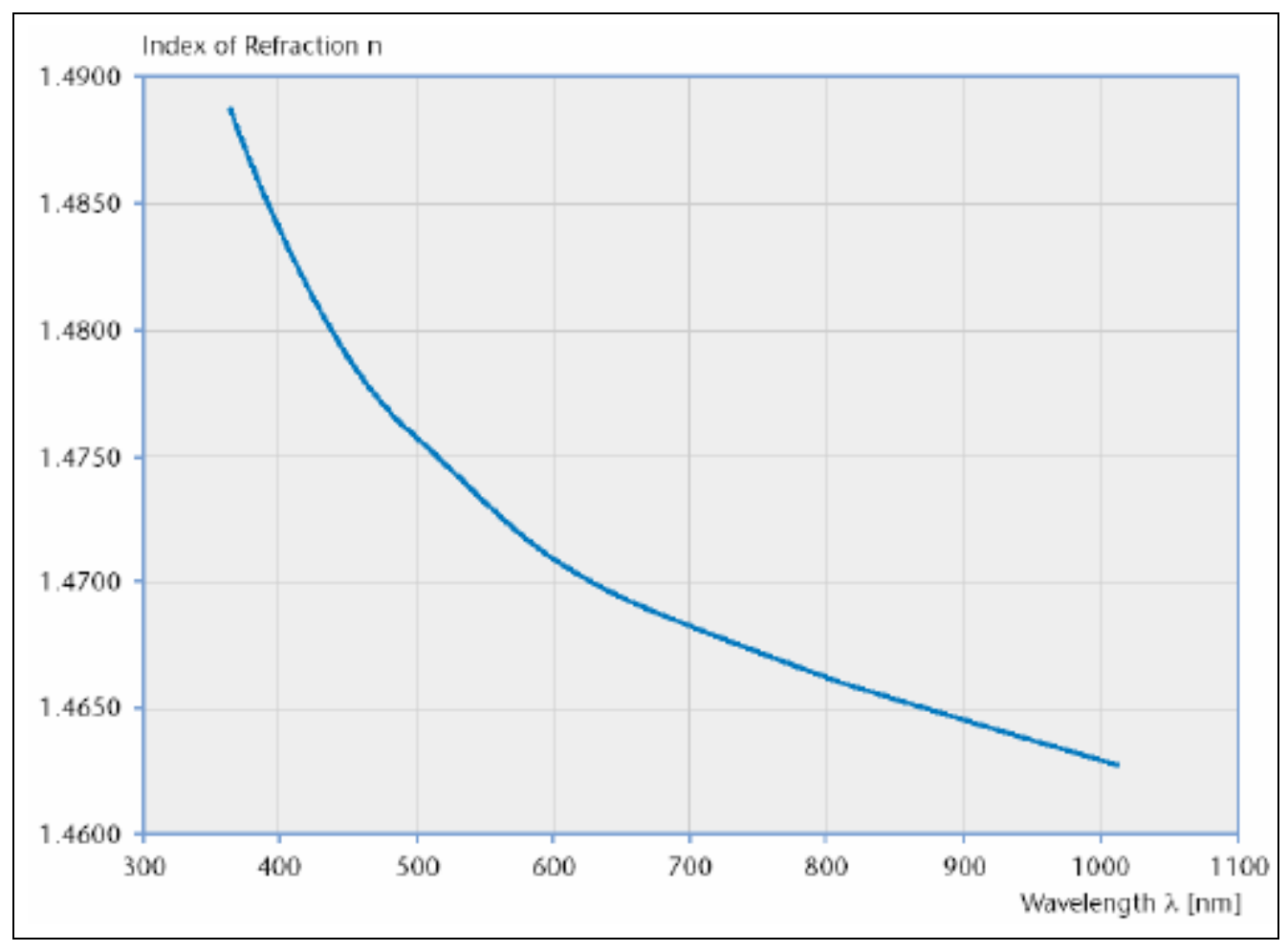

Figure 35 Borofloat index of refraction vs. wavelength [18] 


\section{Chapter 5}

\section{Objective Characterization}

Figure 36 displays the setup used to study the lens.

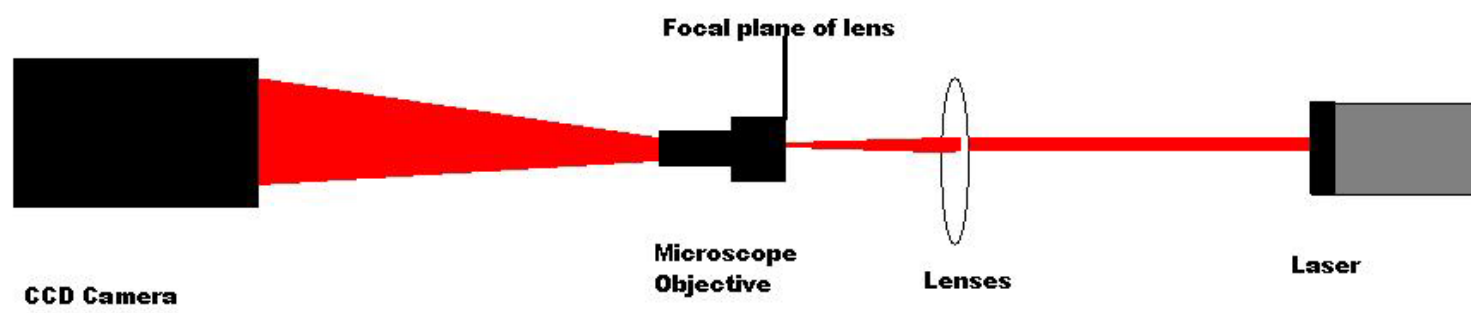

Figure 36 Setup used to take lens characterization measurements

The spot size of the lens is approximately 5 micrometers in diameter. This is smaller than the $11.5 \times 27$ um pixel size of the camera. This necessitated the use of a microscope objective to project an image of the lens spot to a measurable size. The microscope objective was placed in the focal plane of the lens to measure the focal point of the lens. Placing the camera farther from the objective provides a larger projection of the spot. The workbench limited this distance to approximately 1.5 meters which proved sufficient. Beam splitters or mirrors could have been used to increase this distance but would introduce more distortion to the beam and were not used for this reason.

The objective introduced slight alignment issues by skewing the spot projection so that the projected spot did not focus to a uniform point. This causes problems with finding the focal point and measuring the beam diameter. The problem was overcome by placing a piece of cardboard in front of the camera and projecting an out of focus spot onto it. The profile of the 
lens can be seen when the objective is moved 2-3 mm out of the lens focal plane. The profile converges to a point when the objective is moved toward the focal plane and merges with the unfocused laser light when moved away from the focal plane. The unfocused light is present because the beam diameter is larger than the lens. The beam outside the lens propagates straight through the borofloat while the rest gets focused by the lens.

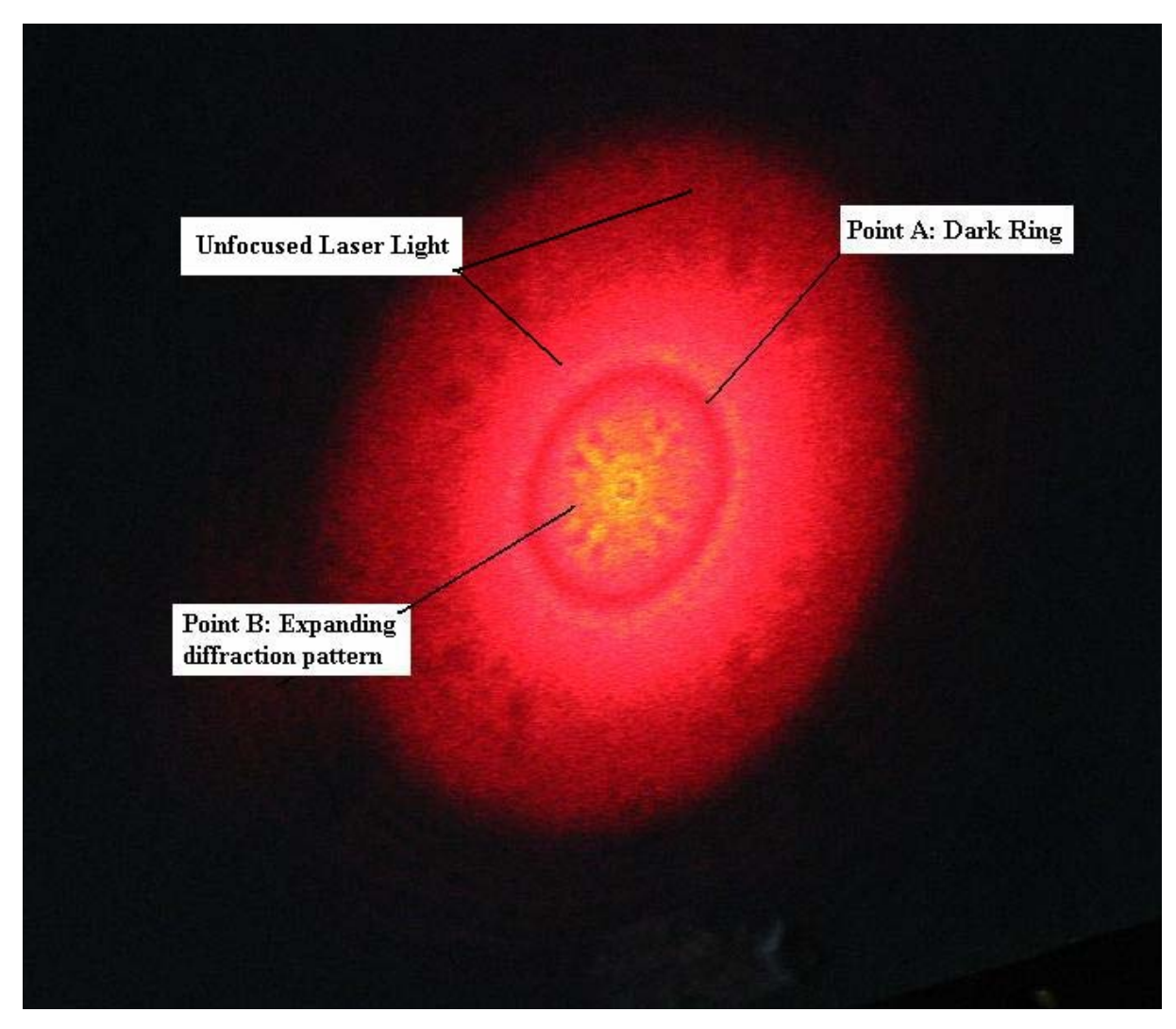

Figure 37 Lens and Microscope Objective alignment

The projected spot can be centered by moving the lens out of focus and changing the objectives angle until the image of the lens merges uniformly with the unfocused laser light.

The other item to consider when using an objective is the magnification introduced. This is crucial to the accurate determination of the spot diameter. Even a deviation of \pm 1 in the magnification value can lead to a deviation of $\pm 0.1-0.3 \mathrm{um}$ in calculating the diameter of the spot. 
The light from the objective diverges over distance which causes the magnification needed to view the spot produced by the lens. To determine the magnification, an object (on the order of the lens size) of known width was placed in line with the laser so that it projects an image of itself onto the CCD camera. This image can then be measured in the Spiricon software and divided by the actual size of the object to get the magnification factor. The object used to determine the magnification was a piece of glass that had a micron scale ruler etched onto its' surface, such as those used in microscopes. Figure 38 is the image of two ruler marks from that substrate. The marks are 100 micrometers apart. The previous method for measuring distances was the same except that the metric used was the width of the letter L which was etched onto the lens wafer. The ruler was used to get a more accurate magnification factor measurement.

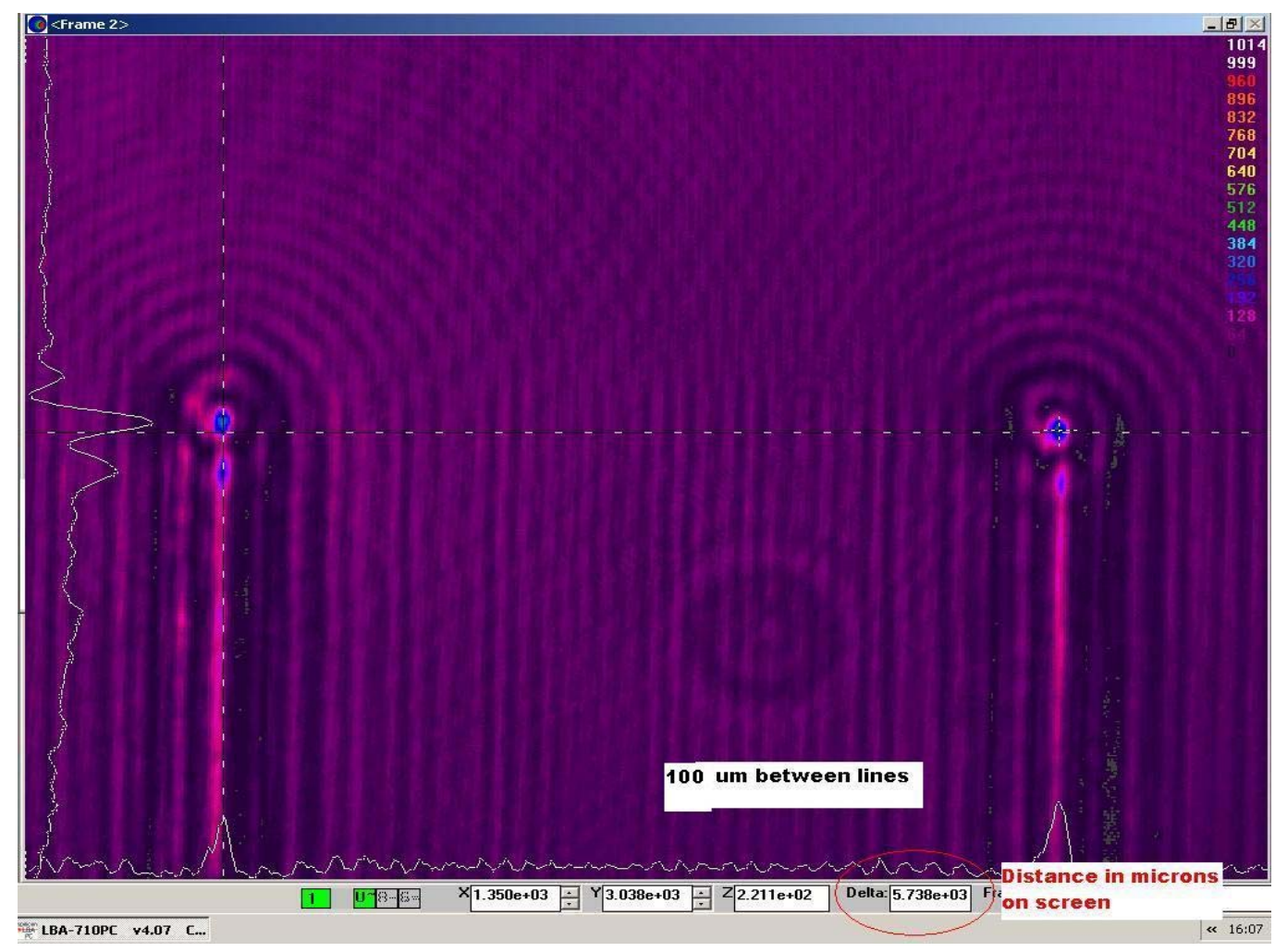

Figure 38 Ruler markings used to calculate objective magnification

The marks in Figure 38 were measured with the Spiricon software to be 5738um. Dividing the software measurement by 100 um gives a magnification of 57.83 which agrees with 
similar, less accurate measurements. This magnification was measured when the camera was approximately 1.73 meters from the objective. The distance between the ruler marks was verified by placing the ruler under the vibrometer on a moveable stage containing micrometer knobs and viewing it on a computer screen. The spacing between ruler marks was then measured by moving one mark across the screen until it occupied the location of a previous mark. A micrometer reading of the distance traveled was taken and used as the distance between marks. 


\section{Chapter 6}

\section{Electromagnetic Propagation and Optical Lens Theory}

The following discussion is adapted from previous optics texts[4,8]. Electromagnetic (EM) waves are phenomenon that consist of traveling energy stored in the form of oscillating electric and magnetic fields. Visible light is a form of EM wave that has electric and magnetic components oscillating at frequencies between $7.69 \times 10^{14}-3.95 \times 10^{14} \mathrm{~Hz}$ or $390-$ $760 \mathrm{~nm}$ in wavelength. Many lasers emit light that is in this range of frequencies and can reach into the infrared and ultraviolet region as well. Optical frequencies can range from $3 \mathrm{x}$ $10^{11}-3 \times 10^{16} \mathrm{~Hz}$. Laser light, being Gaussian and therefore paraxial in nature, can be locally approximated as a Transverse Electro Magnetic (TEM) wave. A TEM wave is defined as an EM wave whose electric and magnetic field components are perpendicular to each other and the direction of propagation. This propagation direction is the direction of power flow and must be parallel or only form small angles to the optical axis to be a TEM wave. There is much theory that is understood about TEM waves. Knowing that laser light is of this form allows us to use that theory to predict and understand laser light better.

There are several layers of theory to predict the propagation, properties, and interactions of EM phenomenon. Some of theses layers are simpler subsets of others while some are merely a different useful way of describing EM behavior. The following is a diagram outlining the subsets of EM theory. 


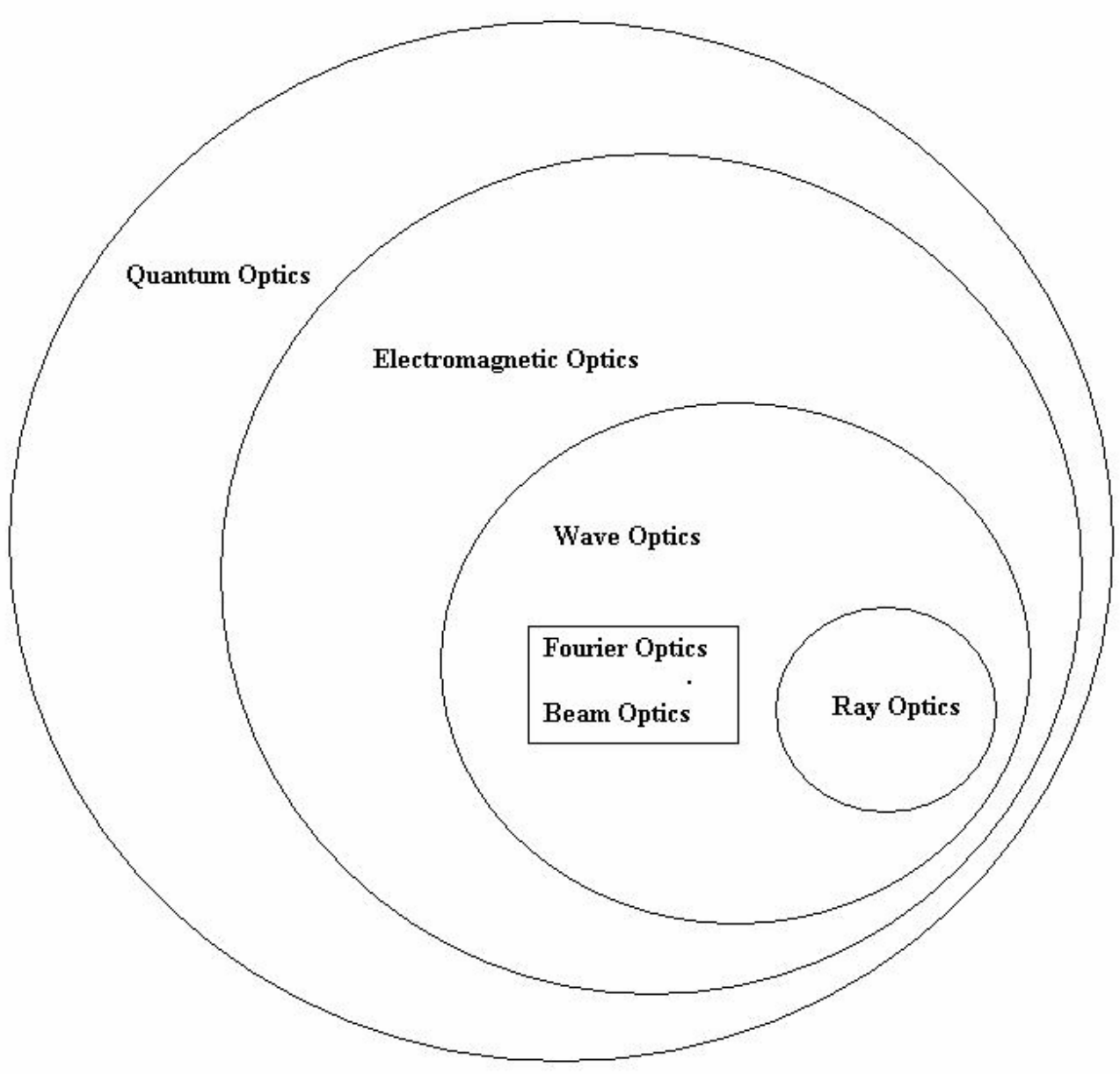

Figure 39 Progression of optics theories from simplest (Ray optics) to most complex (Quantum Optics)

The ray optics theory is the most simple while electromagnetic theory is the most complex description of EM waves. Beam and Fourier theory are additional side-lights to wave theory. Beam theory describes EM wave properties that pertain to directed beams, particularly Gaussian beams. Fourier optics theory describes waves using harmonic analysis and linear systems. The area of electro-magnetics is a very large, complex, and diverse field. The following sections briefly describe a small portion of those theories that are most useful for this thesis. Other informative background can be obtained from[4,8], while theory used to construct the lenses can be found in previous work[1].

\subsection{Ray Theory}

Ray optics theory describes the propagation of light by describing it using vectors and geometry. It gives only the direction and magnitude of the light. Ray optics can be applied 
when the wavelength of the light is much smaller than surrounding objects it interacts with. Some of these postulates require that the medium be homogeneous or inhomogeneous. A homogeneous medium is one in which the refractive index, and therefore the speed of light, is the same in all directions. This theory can describe the propagation of light, its reflection and refraction from simple surfaces, and transmission through simple optical components.

\subsubsection{Snell's Law and Law of Reflection}

Snell's Law describes the refraction (transmission) of a light at the boundary of two different media with refractive indexes $\mathrm{n}_{1}$ and $\mathrm{n}_{2}$. This refraction is determined from the angle of the incident beam and the refractive indexes of the two media[4].

$$
\mathrm{n}_{1} \sin \theta_{1}=\mathrm{n}_{2} \sin \theta_{2}
$$

The law of reflection states that: A reflected ray lies in the plane of incidence and forms the same angle with respect to the boundary normal as that of the incident ray.

\subsection{Wave Theory}

Wave theory is a more complex and informative theory on the nature of light and its interactions with different media. Wave theory can be used when the wavelength of light approaches the size of the surrounding objects with which it interacts. This theory allows us to investigate phenomenon that are unexplainable using ray theory, such as diffraction and interference.

\subsection{Interference and Diffraction Theory}

There are times when EM waves coming from one direction encounter EM waves that are traveling from another direction. This can occur for many reasons, one of which is when an incident EM wave strikes a surface, causing reflections. These reflections can either be constructive (additive) or destructive (subtractive) to the amplitude of the incident wave. Two monochromatic plane waves can be added together such that

$$
\mathrm{U}(\mathrm{r})=\mathrm{U}_{1}(\mathrm{r})+\mathrm{U}_{2}(\mathrm{r})
$$


The intensities of their individual waves are $\mathrm{I}_{1}=\left|\mathrm{U}_{1}{ }^{2}\right|$ and $\mathrm{I}_{2}=\left|\mathrm{U}_{2}{ }^{2}\right|$. The intensity of the resulting wave is

$$
\mathrm{I}=|\mathrm{U}|^{2}=\left|\mathrm{U}_{1}{ }^{2}+\mathrm{U}_{2}{ }^{2}\right|=\left|\mathrm{U}_{1}{ }^{2}\right|+\left|\mathrm{U}_{2}{ }^{2}\right|+\mathrm{U}_{1}{ }^{*} \mathrm{U}_{2}+\mathrm{U}_{1} \mathrm{U}_{2}{ }^{*}
$$

This shows that the intensity of the addition of two plane waves is not the sum of their intensities. Using $\mathrm{U}_{1}=\mathrm{I}_{1}{ }^{1 / 2} \exp \left(\mathrm{j} \varphi_{1}\right)$ and $\mathrm{U}_{2}=\mathrm{I}_{2}{ }^{1 / 2} \exp \left(\mathrm{j} \varphi_{2}\right)$ we get

$$
\mathrm{I}=\mathrm{I}_{1}+\mathrm{I}_{2}+2\left(\mathrm{I}_{1} \mathrm{I}_{2}\right)^{1 / 2} \cos \varphi \quad \varphi=\varphi_{2}-\varphi_{1}
$$

The additional term is the interference of the two waves and is most noticeable when working with coherent (synchronous) light.

Diffraction occurs when an incident light beam encounters an aperture that has dimensions comparable to the wavelength of the incident light. The simplest theory of diffraction is that light traveling though the aperture is unchanged at points within the aperture and only light traveling through the aperture is transmitted. This can be shown mathematically as the aperture function

$$
p(x, y)= \begin{cases}1 & \text { inside the aperture } \\ 0 & \text { outside the aperture }\end{cases}
$$

and

$$
f(x, y)=U(x, y) p(x, y)
$$

We can obtain the output function $\mathrm{g}(\mathrm{x}, \mathrm{y})$ using previous linear systems methods. The output intensity created by the diffraction pattern $\mathrm{I}(\mathrm{x}, \mathrm{y})=|\mathrm{g}(\mathrm{x}, \mathrm{y})|^{2}$ can be either Fraunhofer or Fresnel diffraction, depending on which approximation of free space is used. The case using the Fresnel approximation for free space was used in the DOE design. 


\subsubsection{Huygens-Fresnel Principle}

Diffraction can be more rigorously described as the pattern produced by the interference of multiple waves rising from an incident wave upon an obstruction. The Huygens-Fresnel principle states that each of the unobstructed points of a wave front act as a source for a spherical wave and the superposition of all of these spherical wavelets forms the amplitude of the optical wave at any point beyond the original wave. By taking each infinitesimally small point on the original incident wave as a spherical source, we can see that the next wave front, perpendicular to the direction of propagation and being a sum of these spherical waves, is in phase. This could be intuitively or geometrically discerned from the symmetry of equally spaced spherical waves having wave front normals that are in phase for a given direction. The symmetry of spherical waves in addition to the periodicity of traveling waves can also cause wave fronts that form some angle $\theta$, normal to the incident wave, to be in phase as well. These are referred to as diffractive waves along this direction. The diffraction pattern in the observation plane a distance $\mathrm{z}$ from the aperture, is the sum of all the waves that are formed from points in the aperture.
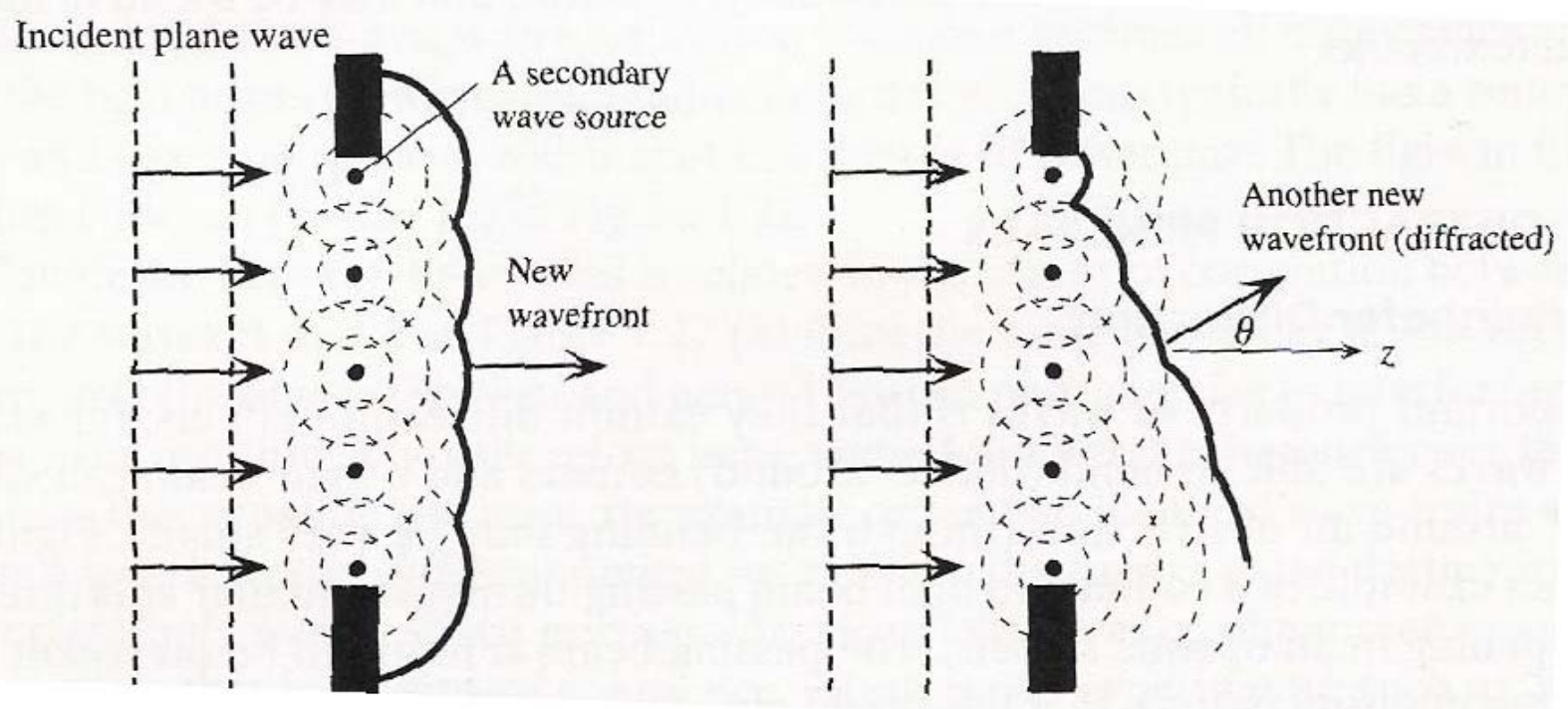

Figure 40 Diagram displaying Fresnel-Huygens principle[8] 
Using a single aperture, we can consider two emitted waves from this aperture in an arbitrary direction $\theta$. The phase of the first emitted wave (A) is formed by a point source $y=0$ at the aperture. The second wave (Y) is formed from a point source at an arbitrary location y in the aperture. If $\mathrm{k}=2 \pi / \lambda$ is the wave number, then $\mathrm{Y}$ is out of phase with respect to $\mathrm{A}$ by $\mathrm{ky} \sin \theta$. The field resulting from the wave emitted from point source at y is[8]

$$
\delta E \propto(\delta y) \exp (-j k y \sin (\theta))
$$

All of the waved from sources at points $y=0$ to $y=a$ emanating from the aperture, must be added together (integrated) to determine the field in the observation plane[8]

$$
\begin{aligned}
& E(\theta)=c \int_{y=0}^{y=a} \delta y \exp (-j k y \sin (\theta)) d y \\
& E(\theta)=\frac{C e^{-j \frac{1}{2} k a \sin (\theta)} a \sin \left(\frac{1}{2} k a \sin (\theta)\right)}{\frac{1}{2} k a \sin (\theta)}
\end{aligned}
$$

where $C$ is a constant. We can then find the intensity as being proportional to $|E(\theta)|^{2}[8]$.

$$
I(\theta)=\left[\frac{C^{\prime} a \sin \left(\frac{1}{2} k a \sin (\theta)\right)}{\frac{1}{2} k a \sin (\theta)}\right]^{2}=I(0) \sin c^{2}\left(\frac{1}{2} k a \sin (\theta)\right)
$$

The above equations are for a single aperture. A diffraction grating is created when a number of these individual apertures are placed close to each other so that the distance $\mathrm{d}$ between apertures is much smaller than the width a of each aperture. We will also assume that the incident light is normal to the surface so that the apertures behave as coherent sources when a beam is incident on them. Waves from neighboring apertures that form an arbitrary angle $\theta$, with respect to the propagation direction of the incident light, are out of phase by an optical 
path difference of $d \sin \theta$. Waves from neighboring apertures will be interfere constructively when this value is an integer multiple of the wavelength so that [8]

$$
\mathrm{d} \sin (\theta)=\mathrm{m} \lambda \quad \mathrm{m}= \pm 1, \pm 2, \ldots
$$

This is known as the grating equation. The diffraction grating provides a way to deflect incoming light. A form of this, the Fresnel Zone Plate, is capable of focusing incoming light.

\subsubsection{Fresnel Zone Plate}

The zone plate is an optical device similar to traditional optical components and can perform many of the same functions as lenses and mirrors depending on their construction. The aim of these optical components is to change the direction of an incident light beam to either focus or diffuse it. Zone plates can focus (reflect) light but do this using diffraction and interference whereas bulk lenses (mirrors) use refraction (reflection).

\subsubsection{Zone Plate Description}

The following discussion is adapted from previous zone plate texts[10]. The zone plate is constructed by making alternating clear and opaque concentric circles and can be viewed as a generalization of pinhole optics. A pinhole aperture has the advantages of: virtually infinite depth of field, wide angular field, and no linear distortion. Its drawbacks are low resolution and low light gathering ability. Zone plates are able retain some of the advantages of pinhole optics while overcoming some of the disadvantages. A zone plate can even have as good a resolution as a traditional lens of the same aperture size if it is illuminated with a relatively monochromatic light source and the plate has the right number of zone rings. Zone plates are also able to be used at wavelengths where traditional optics cannot, such as X-Ray or UV wavelengths.

There are different types of zone plates that vary based on the formulas used to conceptualize them[21]. Each type still has the underlying principle of alternating opaque and clear regions. A Fresnel zone plate is defined as having alternating clear and opaque regions of constant area 
equal to $\pi \lambda f, \lambda$ is the wavelength of an incident light wave, and $f$ is the principle focal length of the zone plate. It gives sharp zone boundaries that are defined as $r_{n}=(n \lambda f)^{1 / 2}$ [23]. A square wave is produced if the amplitude transmittance is plotted against the radius. In addition to the primary focus, there are secondary foci at $\mathrm{f} / 3, \mathrm{f} / 5, \mathrm{f} / 7$ etc.

Zone rings can be constructed with different phase profiles[22]. These profiles can range from a continuous profile that has an efficiency of $100 \%$, to a binary profile with an efficiency of $40.5 \%$, and any number of profile levels in between. The profile used is a factor in how much of the incident light is focused to the primary focus by the zone plate. The continuous profile can be achieved by thinking of each ring as a concentric circle with radius $r_{n}=\left(n \lambda f+n^{2} \lambda^{2} / 4\right)^{1 / 2}$, where $n$ is the refractive index, $f$ the focal length, and $\lambda$ is the wavelength of the incident light. If we truncate this to $r_{n} \approx(n \lambda f)^{1 / 2}$, we get the binary profile.

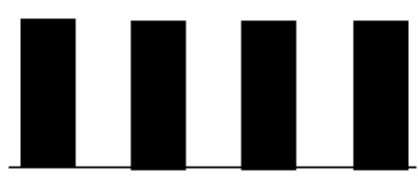

Binary profile

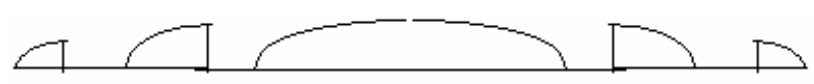

Continuous profile

Figure 41 Binary and Continuous profiles of a Fresnel Zone Plate

\subsubsection{Material Selection and Fabrication Techniques}

Zone rings can be made out of any variety of materials depending on the application of the lens and wavelength of the incident light. Some materials are opaque to certain wavelength ranges and not in others (ex. silicon will not transmit wavelengths that are greater than 1um). This happens because the band gap of a material will only absorb photons with energies that are equal to or larger than the band gap. This means that a given material will only transmit light with a certain wavelength or longer. This allows the zone plate to operate in many different frequency ranges depending upon the material used in the construction of the zone 
rings. There are many different combinations of masks, resist materials, and etching methods that are used to create zone plates. The method used depends on: the application of the zone plate, required precision, available money, and time for the production of the zone plate. More information about fabrication can be found in [7], while [1] contains the fabrication process used to create the diffractive lenses described in this thesis. 


\section{Chapter 7}

\section{DOE Lens Modeling and Design}

Previous efforts [1] were successful in designing and modeling diffractive Fresnel lenses. The modeling and design used a linear systems approach using the Fresnel approximation for the transfer function of free space. The complex two dimensional incident and transmitted beams were calculated, then the transfer function of free space. The transmitted beam and transfer function were converted to the frequency domain, multiplied, then converted to spatial domain to calculate the lens aperture shadow. This aperture shadow was then divided by the input beam to obtain the lens array. This array was then for depth to obtain the diffraction profile of the Fresnel diffractive optical element. The profile was then converted into a binary pattern for easier fabrication. This whole process was completed in Mat-Lab. The program written[1] is capable of taking various values of refractive index, lens radius, focal length, desired output spot size, etc. to calculate the diffractive pattern needed. Functions were also written[1] to convert the Mat-Lab data to a format useable by L-Edit so it could be exported to a format useable in the mask generation process. 


\section{Chapter 8}

\section{Lens Characterization}

\subsection{Test Setup and Lens Description}

The diffractive optical lenses being characterized here are Fresnel lenses that have been etched into borofloat glass. Previous works was done to develop a Mat-lab program to produce a 2-D layout of the lenses, a mask made from that lens design that was used in a photolithography process, and the lenses were etched into a borofloat wafer. Fabrication procedures were devised and a set of lenses were fabricated that are being characterized in this thesis. The intent of these lenses is to focus an incoming laser beam to a diameter of 5 um for use in integrated optical monitoring of MEMS devices.

The test lens produces its spot using diffraction whereas a bulk optical lens uses refraction. A diffractive lens alters the course of a light beam by the interaction of the beam and lens at the interfaces and edges of the diffraction grating. This occurs when the features of the lens are near the size of the wavelength of incident light. A refractive lens alters the course of the light because of the change in refractive index between the lens and outside medium or can be found more precisely from the electromagnetic boundary conditions.

The lenses are arranged in a $6 \times 8$ grid as shown in Figure 42. C2R1 is the lens used in the following analysis and is circled in red. The designation used for referring to this lens is C2R1 (Column 2, Row 1 - as viewed from the front of the wafer). The front of the wafer is determined by viewing each side at a slight angle under a room lamp. The front side is being viewed if its reflection can be seen from the bottom of the wafer. 


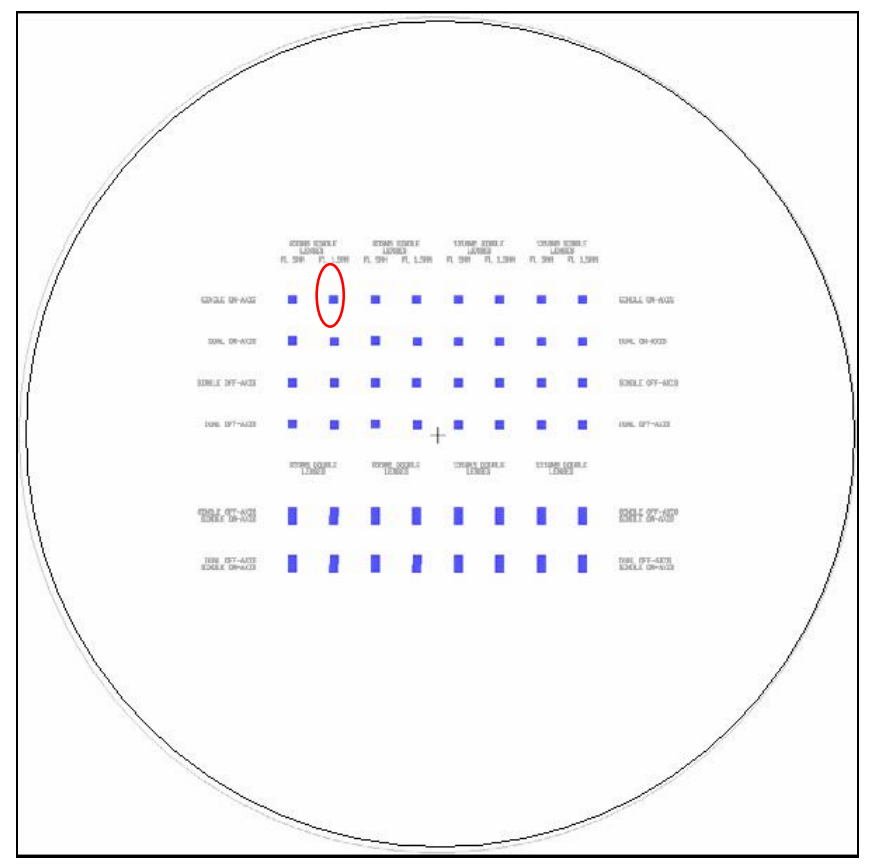

Figure 42 Fresnel lens C2R1 (circled in red) was used for tests [1]

\begin{tabular}{|l|c|c|c|c|}
\hline & $\begin{array}{c}\mathbf{6 3 3 n m} \\
\mathbf{5 m m} \text { FL }\end{array}$ & $\begin{array}{c}\mathbf{6 3 3 n m} \\
\mathbf{1 . 5 m m} \text { FL }\end{array}$ & $\begin{array}{c}\mathbf{1 3 1 0 n m} \\
\mathbf{5 m m} \text { FL }\end{array}$ & $\begin{array}{c}\mathbf{1 3 1 0 n m} \\
\mathbf{1 . 5 m m} \text { FL }\end{array}$ \\
\hline $\begin{array}{l}\text { On-axis, } \\
\text { Single Spot }\end{array}$ & Row 1, Cols 1\&3 & Row 1, Cols 2\&4 & Row 1, Cols 5\&7 & Row 1, Cols 6\&8 \\
\hline $\begin{array}{l}\text { On-axis, } \\
\text { Dual Spot }\end{array}$ & Row 2, Cols 1\&3 & Row 2, Cols 2\&4 & Row 2, Cols 5\&7 & Row 2, Cols 6\&8 \\
\hline $\begin{array}{l}\text { Off-axis, } \\
\text { Single Spot }\end{array}$ & Row 3, Cols 1\&3 & Row 3, Cols 2\&4 & Row 3, Cols 5\&7 & Row 3, Cols 6\&8 \\
\hline $\begin{array}{l}\text { Off-axis, } \\
\text { Dual Spot }\end{array}$ & Row 4, Cols 1\&3 & Row 4, Cols 2\&4 & Row 4, Cols 5\&7 & Row 4, Cols 6\&8 \\
\hline $\begin{array}{l}\text { Dual lens, } \\
\text { Single Spot }\end{array}$ & Row 5, Cols 1\&3 & Row 5, Cols 2\&4 & Row 5, Cols 5\&7 & Row 5, Cols 6\&8 \\
\hline $\begin{array}{l}\text { Dual lens, } \\
\text { Dual Spot }\end{array}$ & Row 6, Cols 1\&3 & Row 6, Cols 2\&4 & Row 6, Cols 5\&7 & Row 6, Cols 6\&8 \\
\hline
\end{tabular}

Table 11 Lens layout[1] 


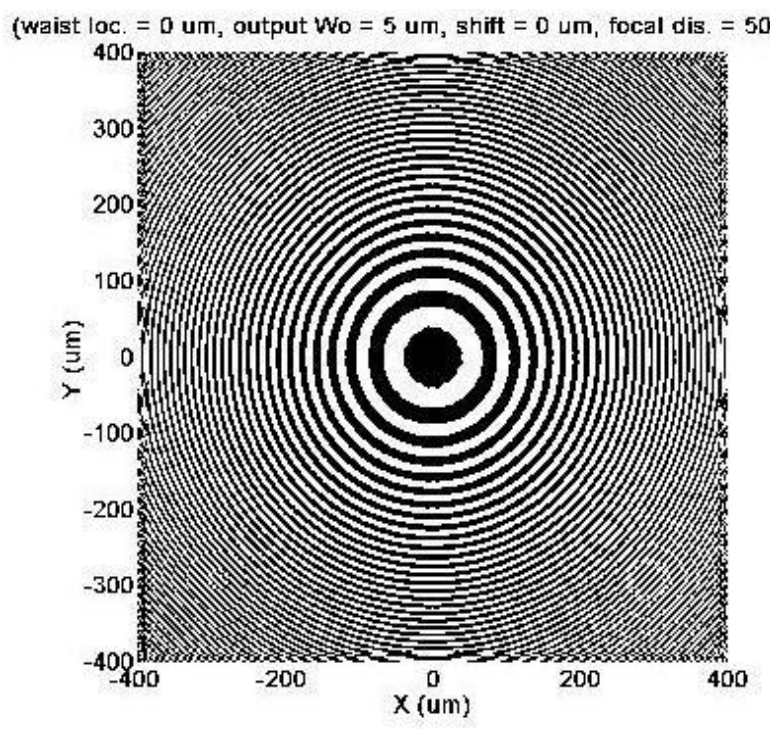

Figure 43 Designed profile of lens C2R1 created by a Mat-lab program[1]

The lens profile was fabricated as a binary pattern instead of a continuous (blazed) one. The binary profile was chosen for ease of fabrication.

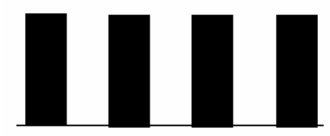

Figure 44 Binary profile

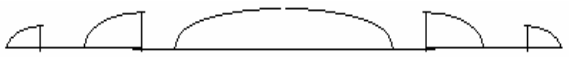

Figure 45 Continuous profile

The binary profile has only two height levels, while the continuous profile has many height levels. Each will affect the incident light in a different way even if the same lens pattern is used.

The HeNe laser characterized earlier was used in the following tests. The previous analysis indicates that the lens should be placed no closer than 0.30 meters from the face of the laser case. This is to avoid any effects of the non-Gaussian behavior observed from this laser at distances closer than 0.30 meters. All tests were performed with the lenses at approximately 
$0.38 \mathrm{~m}$ from the laser and under paraxial conditions unless otherwise specified. This means the incident beam was either normal to the lens surface or made only slight angles to the surface. This is the condition under which the lenses were designed. It should be noted that there was no strict method known at the time that could be used to determine the angle of incidence of the beam. Visual inspection was used to align the laser beam to be normal to the lens. The method used to determine if the beam was normally incident on the lens comes from ray optics. A beam incident on a flat surface forms an angle $\theta_{\mathrm{i}}$ that is equal to the angle $\theta_{\mathrm{r}}$ of the reflected wave if the surface is one that will produce a reflection.

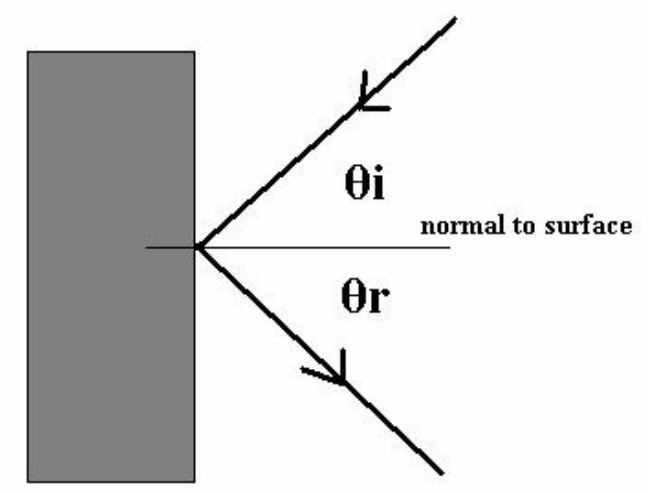

Figure 46 Picture of Snell's Law

The borofloat wafer containing the lenses was tilted until the reflected beam formed a spot close to the aperture of the laser.

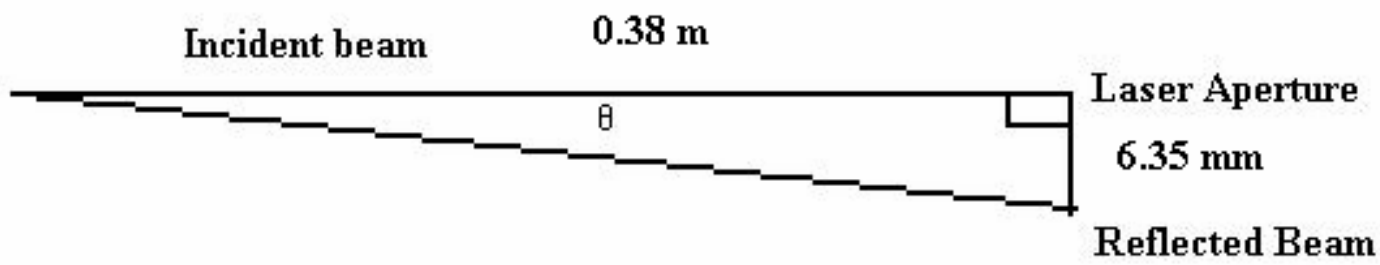

Figure 47 Paraxial Test 
The reflected to incident angle $\theta$ was found to be:

$$
\arctan (0.00635 / 0.381) \approx 0.01667 \mathrm{rad} \approx 0.00635 / 0.381
$$

This angle is sufficient to assume paraxial conditions $(\tan \theta \approx \theta)$. The beam was not reflected back into the laser to avoid unwanted feedback into the laser housing. Measurements were taken using a CCD camera and software from Spiricon.

\subsection{Diameter}

The diameter of the focused spot was designed to be $5 \mathrm{um}$. The diameter was determined by using the $13.5 \%$ peak method instead of the 4 sigma method. Distortion from the lens caused the 4-sigma method to be inaccurate. This is only the case for lenses c2R1 and C4R1 as the other lenses were in better shape. The following pictures show the distortion result on the diameter calculation.
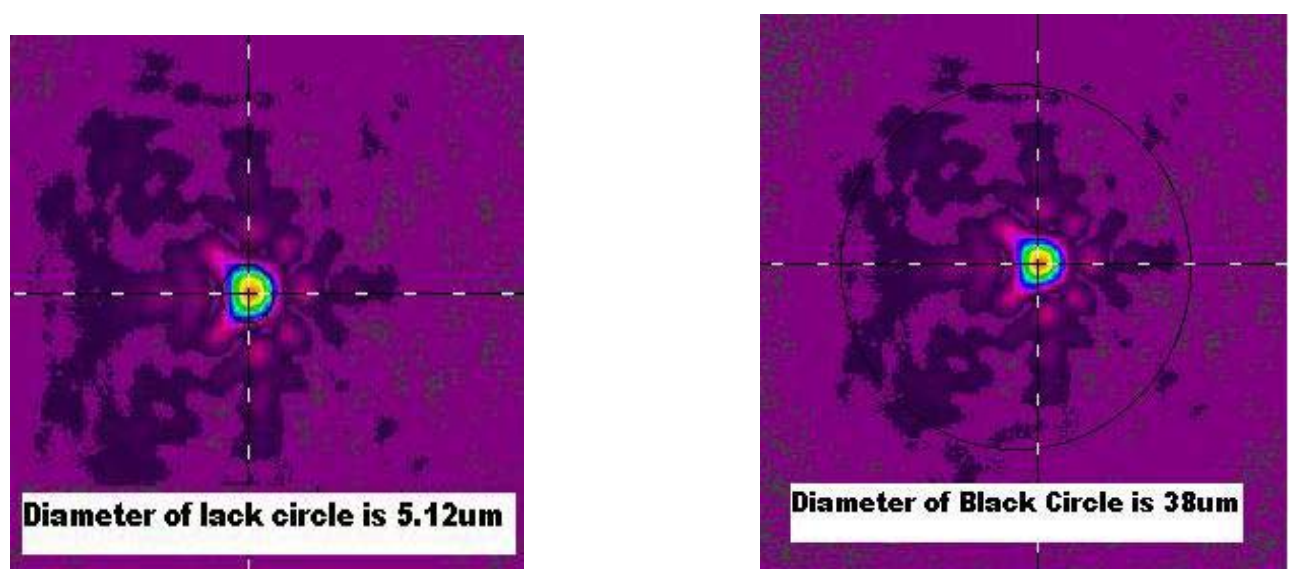

Figure 48 Comparison of $13.5 \%$ and 4 -sigma methods in

Spiricon software to measure lens beam diameter for lens C2R1 
Figure 48 displays the same spot and a black circle outlining the diameter calculated by the Spiricon software. The left picture shows the diameter measured using the $13.5 \%$ method while right picture shows the diameter measured using the 4-sigma method. A magnification factor of 57 was used to calculate the diameters. The outlying dark regions are assumed to be stray distortion from the improperly formed lens. This is largely caused from fabrication errors due to the small feature size of this lens and limits of the equipment used. Secondary causes include: the use of a binary pattern instead of a continuous one, and the use of wave theory to design the lenses. All of these would cause some light to not be cancelled when it should be since they are approximations of more precise methods.

The choice of the spot diameter measurement depends upon the lowest power density required at the spots outer edge, SNR, and efficiency needed for the particular application. Measurements using the $13.5 \%$ method range from $4.5-5.5$ um in diameter. This is highly dependent on: centering the laser on the lens, placing the objective on the exact location of the focal plane of the lens, and ensuring that the output from the objective converges uniformly in forming the spot image.

\subsection{Partial Incidence of Laser on Lens}

The next item in question is how the lens behaves when only part of the laser is incident on it. Figure 49 shows the focal plane of the lens as the laser beam is normal to and moves vertically across the lens. The behavior indicates that partial use of the beam affects the intensity of the beam peak and only slightly alters its shape though maintaining a mostly Gaussian profile. 

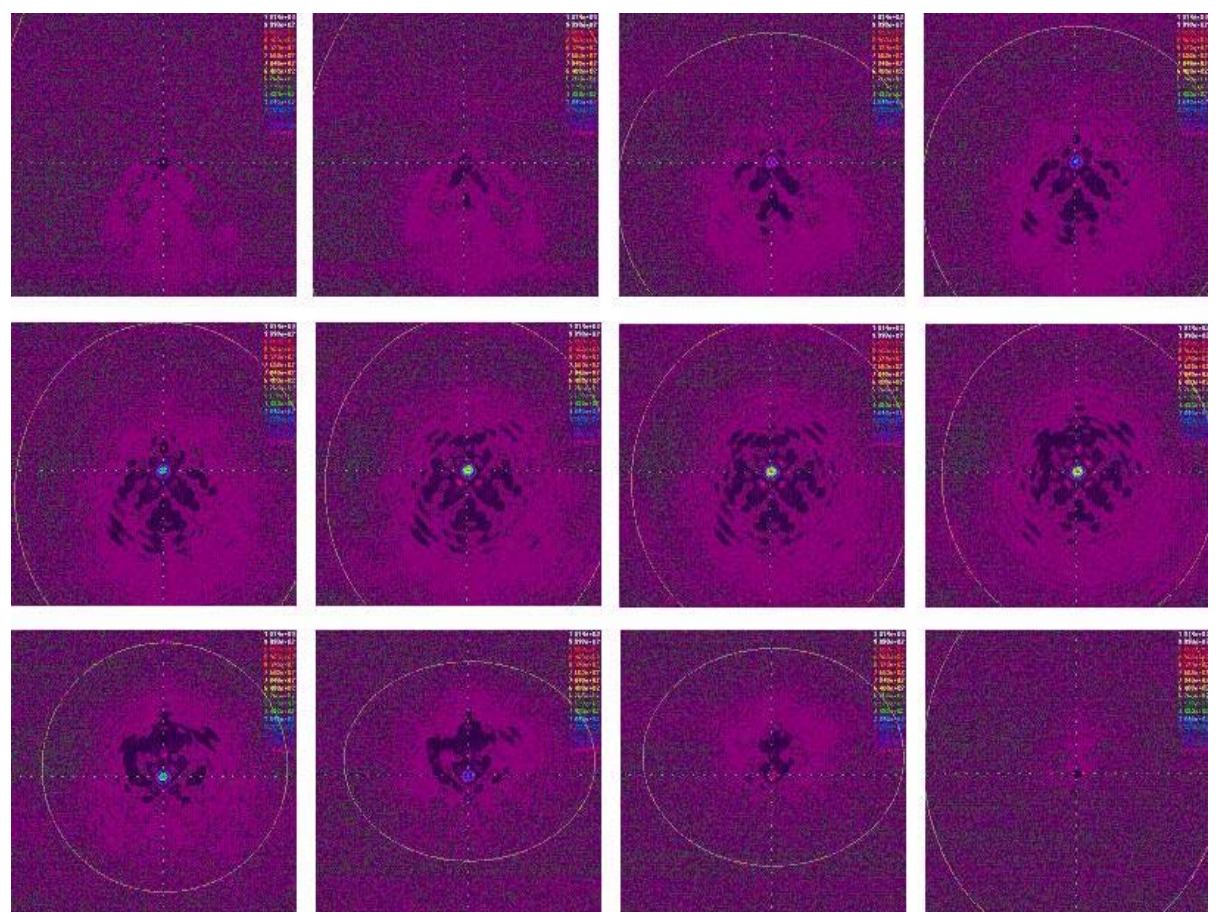

Figure 49 Time lapse pictures of beam passing normal to and vertically across lens

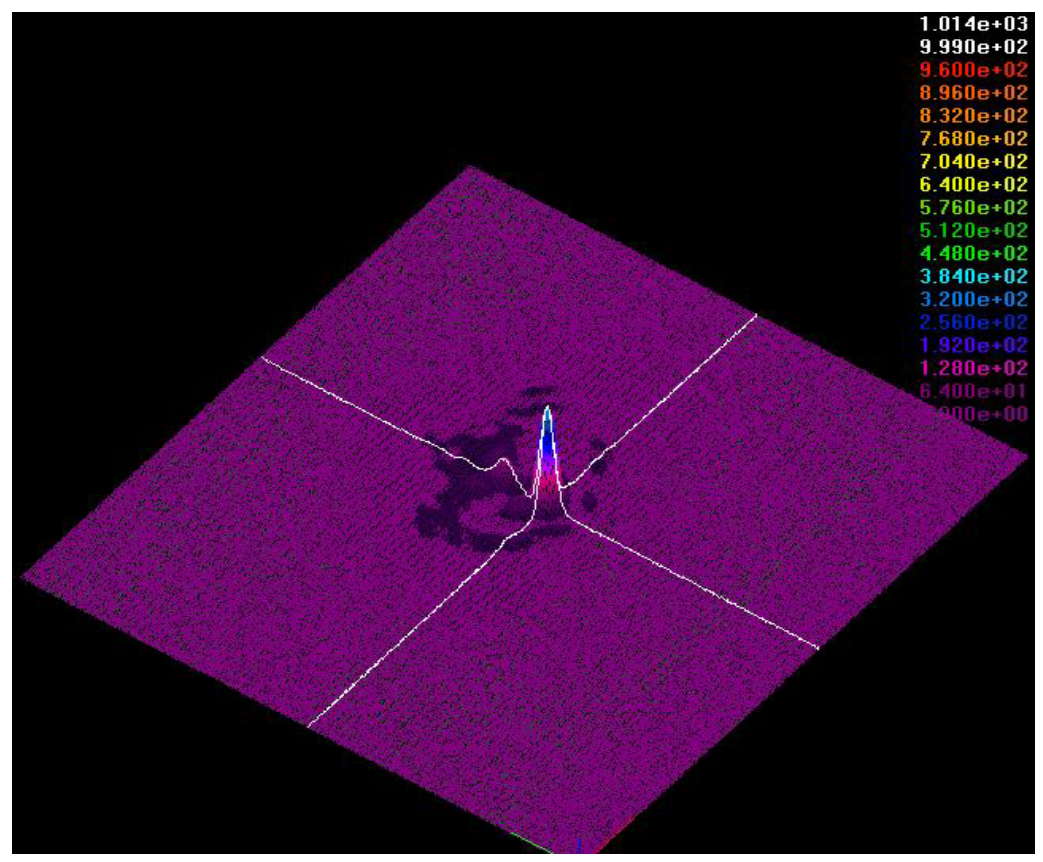

Figure 50 Beam center near top of lens 


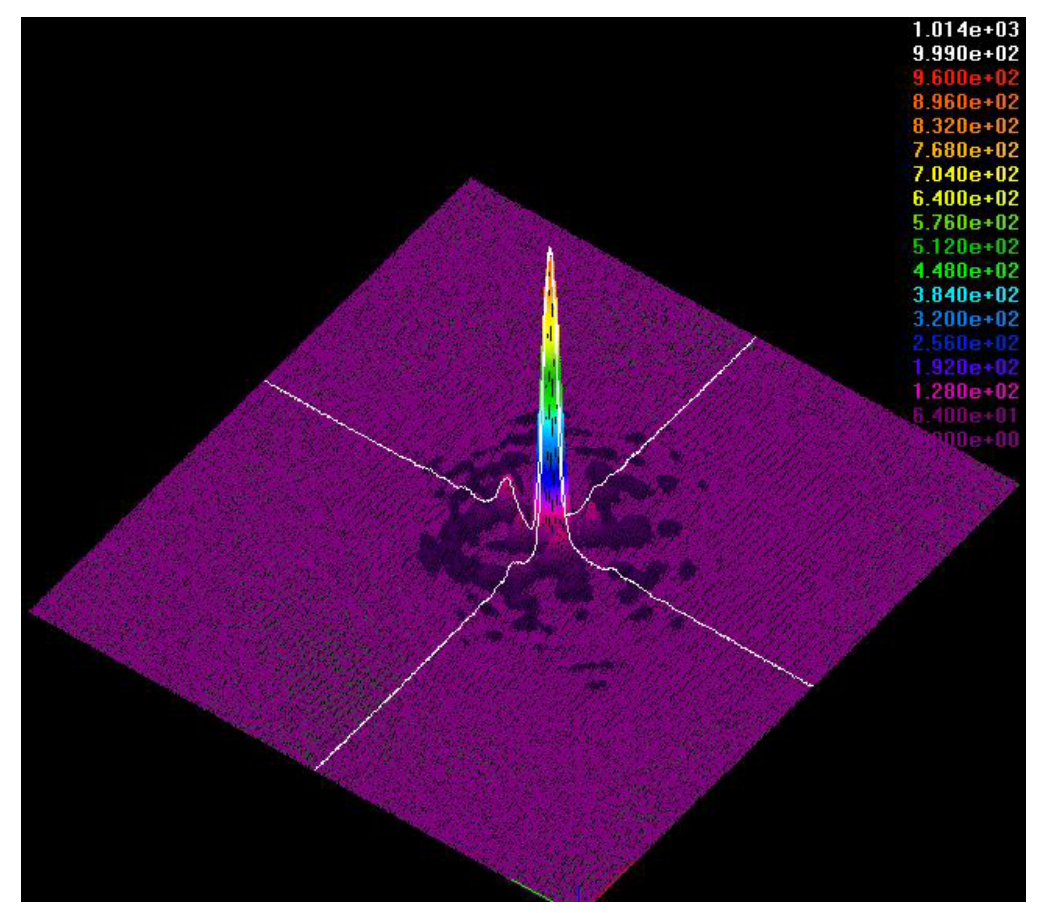

Figure 51 Beam center near center of lens

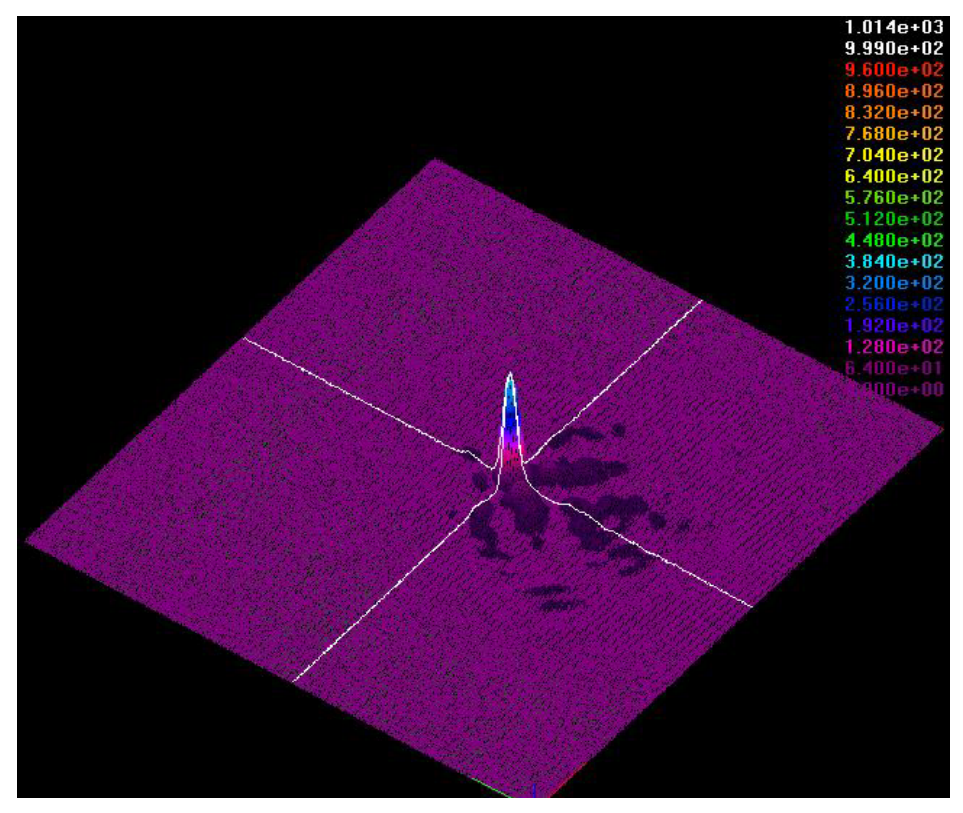

Figure 52 Beam center near bottom of lens

Figure 50 shows the spot formed as the laser travels from the top of the lens. Figure 51 shows the laser centered on the lens. Figure 52 shows the laser traveling past the bottom of the lens. 
These three show how the Gaussian nature of the beam is maintained and amplitude changes as the center of the incident beam moves from the top of the lens to the bottom.

\subsection{Focal Point and Focal Range}

The designed focal distance for lens C2R1 is $5 \mathrm{~mm}$. The measured focal distance was found to be $\sim 5.3 \mathrm{~mm}$ from the back of the borofloat wafer. The wafer and objective were each fixed to the workbench with moveable clamps. The clamp micrometers from Mitutoyo (series 148118) had a graduation of $0.01 \mathrm{~mm}$, accuracy of $\pm 0.002 \mathrm{~mm}$, and range of $13 \mathrm{~mm}$. The objective face was placed on the back of the wafer and slowly moved back until the focal point was reached. The focal point was observed with the CCD camera and Spiricon software. The laser beam was then moved across each axis to ensure that it was centered on the lens. The laser was assumed to be centered on the lens when the observed lens output reached a maximum while moving the laser across the lens. This process was repeated until the maximum beam peak was observed.

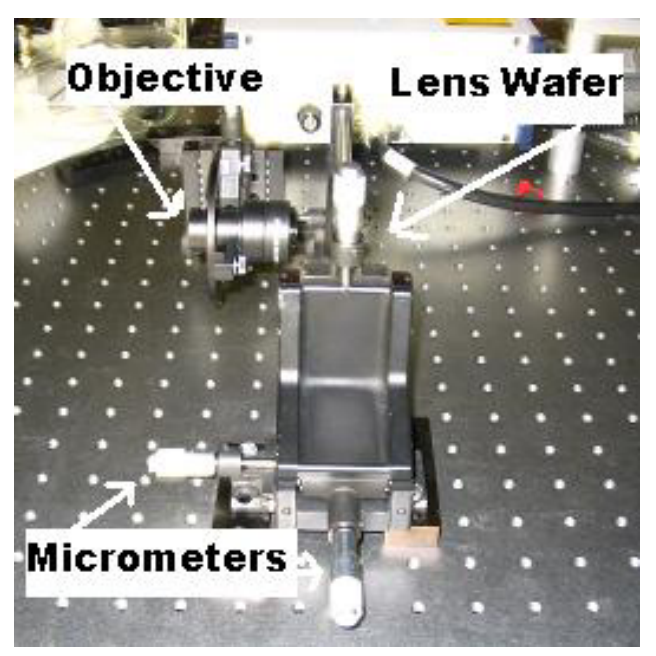

Figure 53 Lab picture of setup used for focal point measurement

A lens has both a focal point diameter and focal range over which the beam stays in focus. The value of one quantity affects the other. A smaller spot size leads to a shorter focal range. A larger spot leads to a longer focal range. Determination of the focal range depends on the acceptable SNR and spot size of the application. 


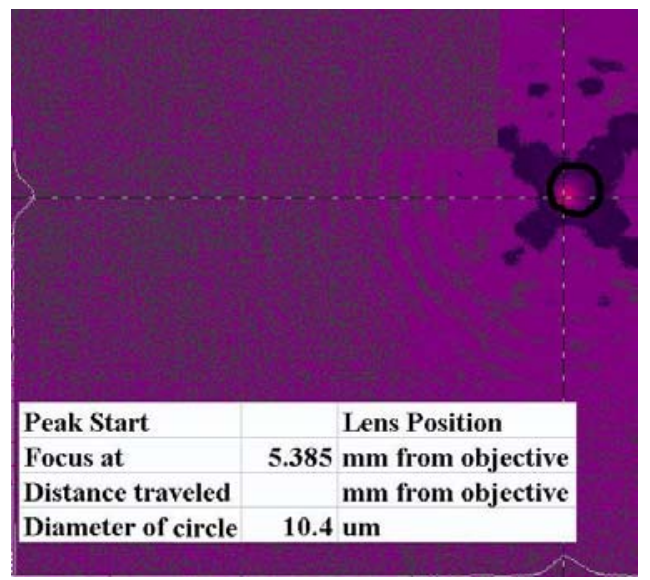

Figure 54 Diameter measured with $13.5 \%$ method at $5.385 \mathrm{~mm}$ from lens

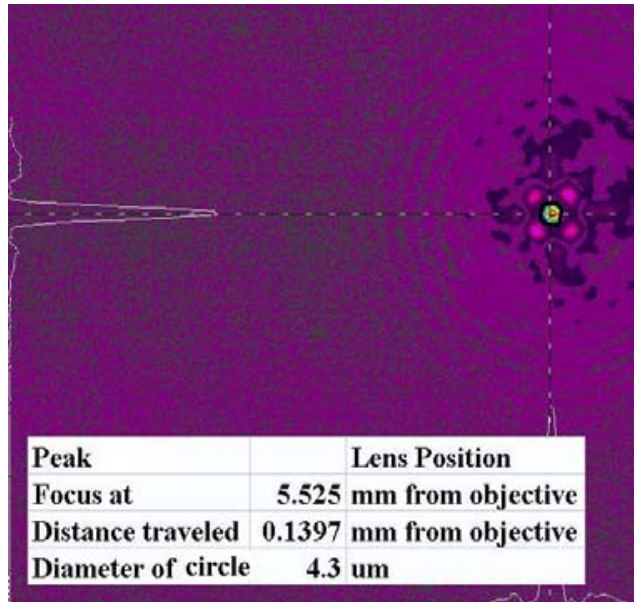

Figure 55 Diameter measured with $13.5 \%$ method at $5.525 \mathrm{~mm}$ from lens

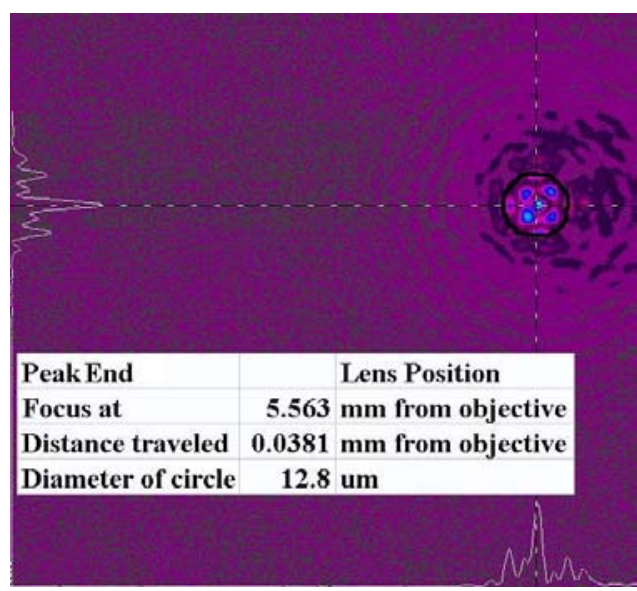

Figure 56 Diameter measured with $13.5 \%$ method at $5.563 \mathrm{~mm}$ from lens 


\subsection{Multiple Peaks}

It was discovered that there are multiple focal points for lenses C2R1 and C4R1. They do not have complete lens patterns. This is likely caused by non-idealities of fabrication and small feature sizes since the other lenses do not experience this. Two focal regions for C2R1 have been identified: one around $5.5 \mathrm{~mm}$ and another around $8.5 \mathrm{~mm}$. Each focal region contains a primary and secondary focal point. approximately $0.1 \mathrm{~mm}$ apart. The secondary focal points are slightly smaller than the primary but have an appreciable amount of noise associated with them.

Figure 57 is the primary peak and Figure 58 is the secondary peak of the $5.5 \mathrm{~mm}$ focal region. The noise around the secondary peak makes it harder to define the peak diameter because the program has difficulty determining the peak location. Manual determination of the diameter in the program is used for the secondary peak by using the software to isolate the region of interest. This should be carefully done so that the two peaks can be compared. The secondary peak diameter appears to be slightly smaller than primary peak upon visual inspection.

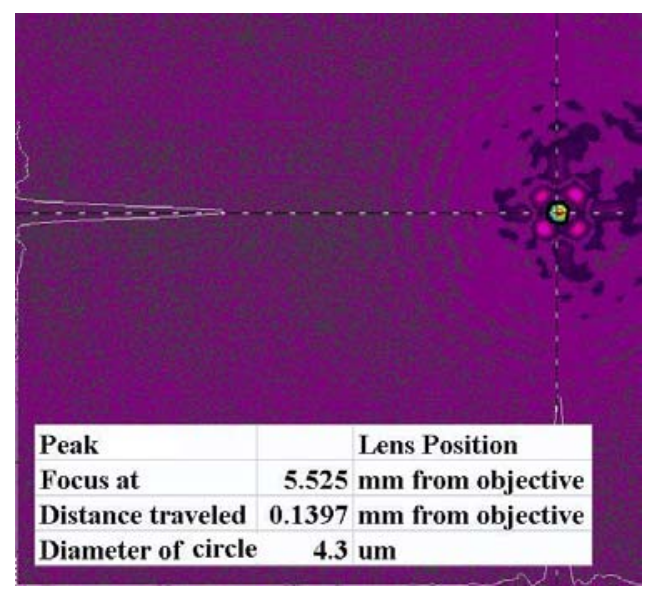

Figure 57 Primary peak of region 1 at $5.525 \mathrm{~mm}$ from lens 


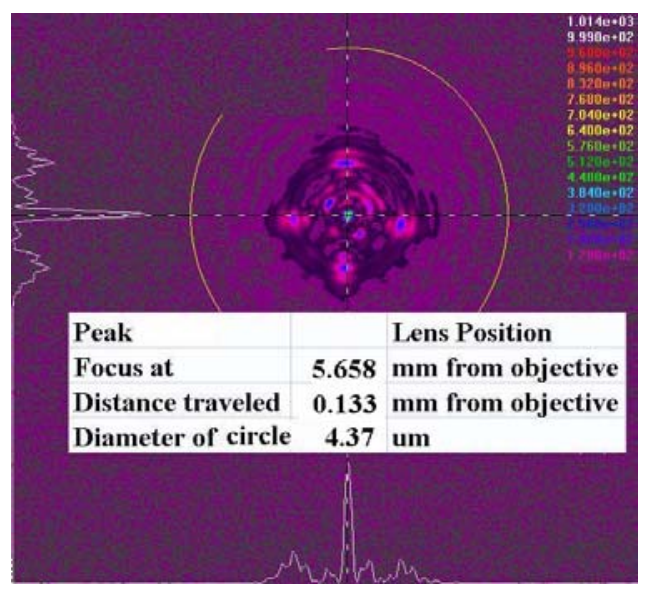

Figure 58 Secondary peak of region 1 at $5.658 \mathrm{~mm}$ from lens

\subsection{Pointing Stability}

The pointing stability through the lenses was conducted. An accurate measure of the laser pointing stability must be taken and other possible vibrations canceled out or accounted for. This laser stability must then be considered in conjunction with the lens stability measurements to see the effects the lens has on the pointing stability. A method for this has not yet been devised. The lens stability tests also introduce more vibration prone items, such as clamps and the objective, that can affect the outcome of the lens stability tests. Figure 59 shows the lens pointing stability test that has not taken potential vibration sources into account.

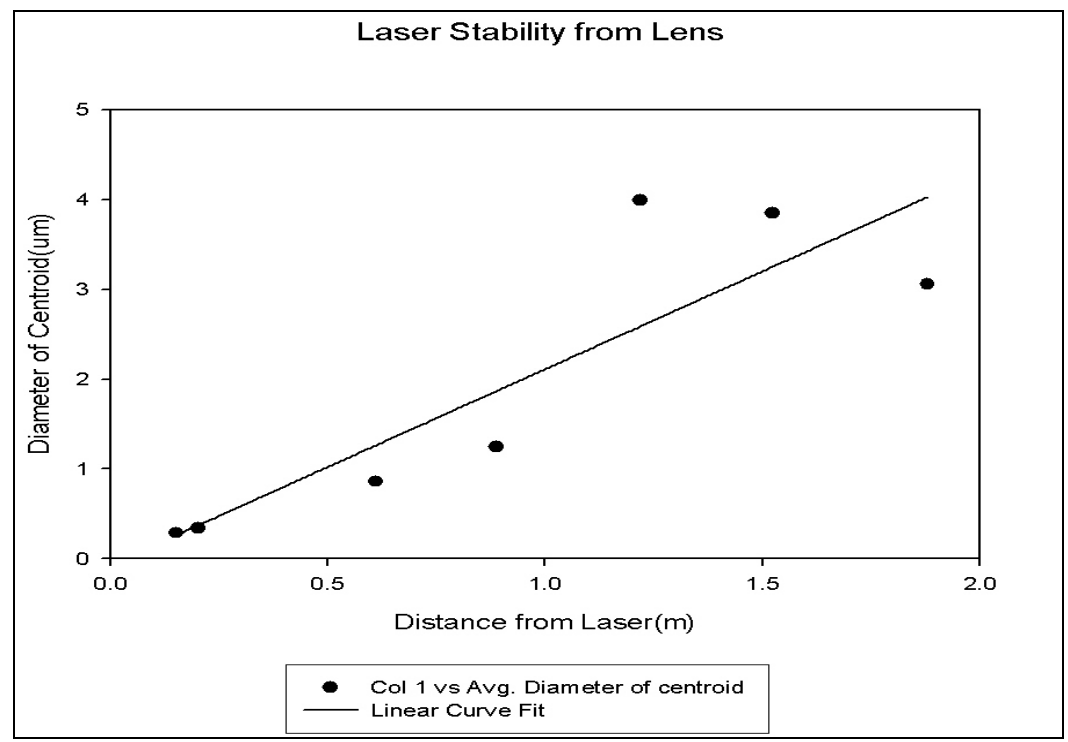

Figure 59 Lens pointing stability measurements 
Each point represents the average laser beam peak location calculated from 125 samples. Except for a couple of outliers, the stability seems to worsen linearly over distance from the laser housing. This is expected since a small instability at a short distance would get amplified over longer distances. These tests are not yet complete.

\subsection{Power Loss, Efficiency, and SNR}

Power loss of the lenses is important to determine efficiency. The size of the lens complicates this measurement. The lenses were placed at a distance of $\sim 0.381$ meters from the laser housing to avoid the non-Gaussian nature of the beam observed at distances less than 0.30 meters from the laser. The lens profile is square with a side length of $800 \mathrm{um}$. The 4-sigma beam diameter at 0.39 meters as measured to be 918 um which places some percentage of the beam outside the lens area. That percentage of the beam should not be considered when calculating power loss since it is not incident on the actual lens area. The Gaussian nature of the beam also makes calculating the incident power more difficult since the beam intensity is not uniform.

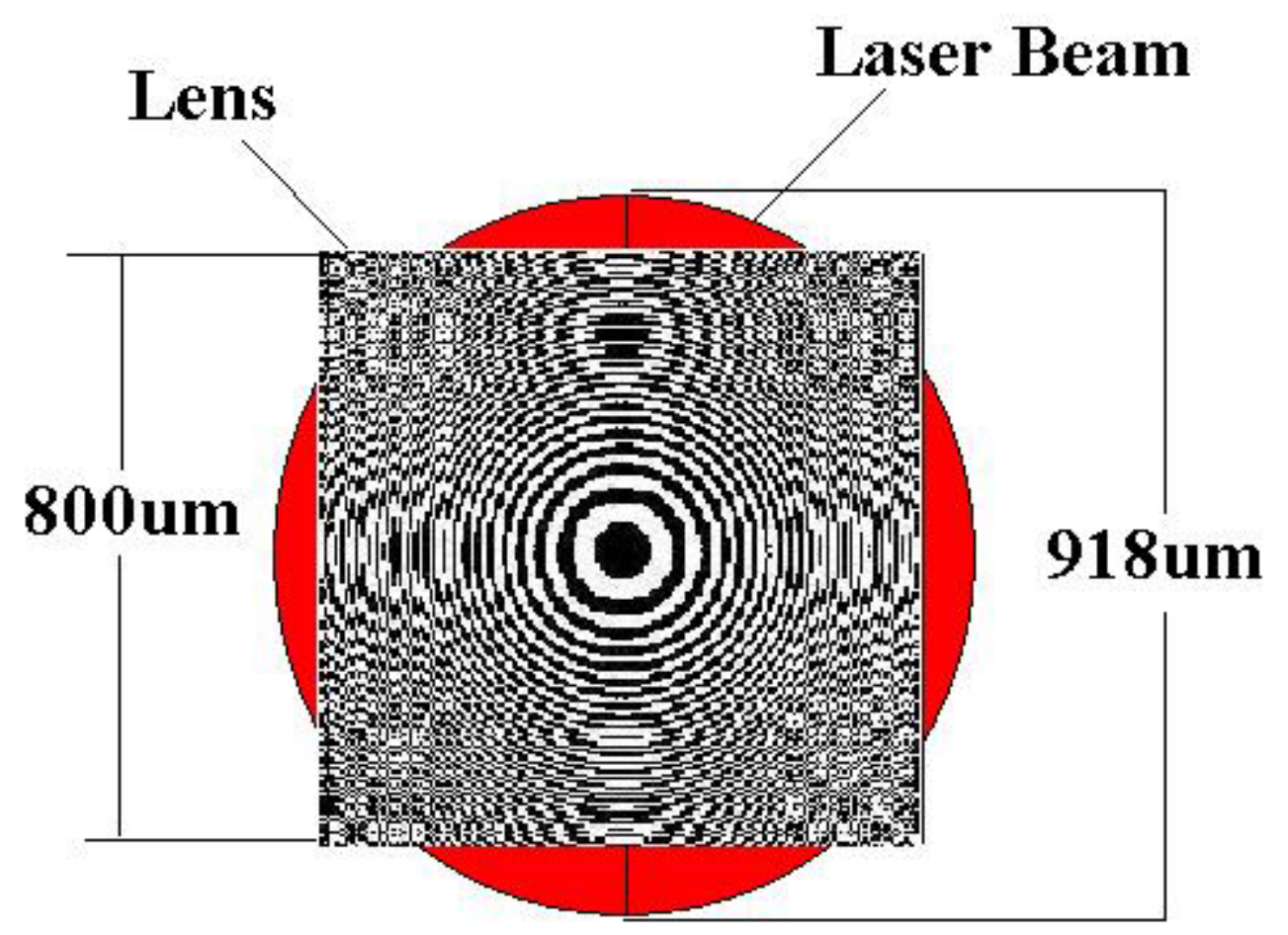

Figure 60 Drawing of lens and laser spot size difference 


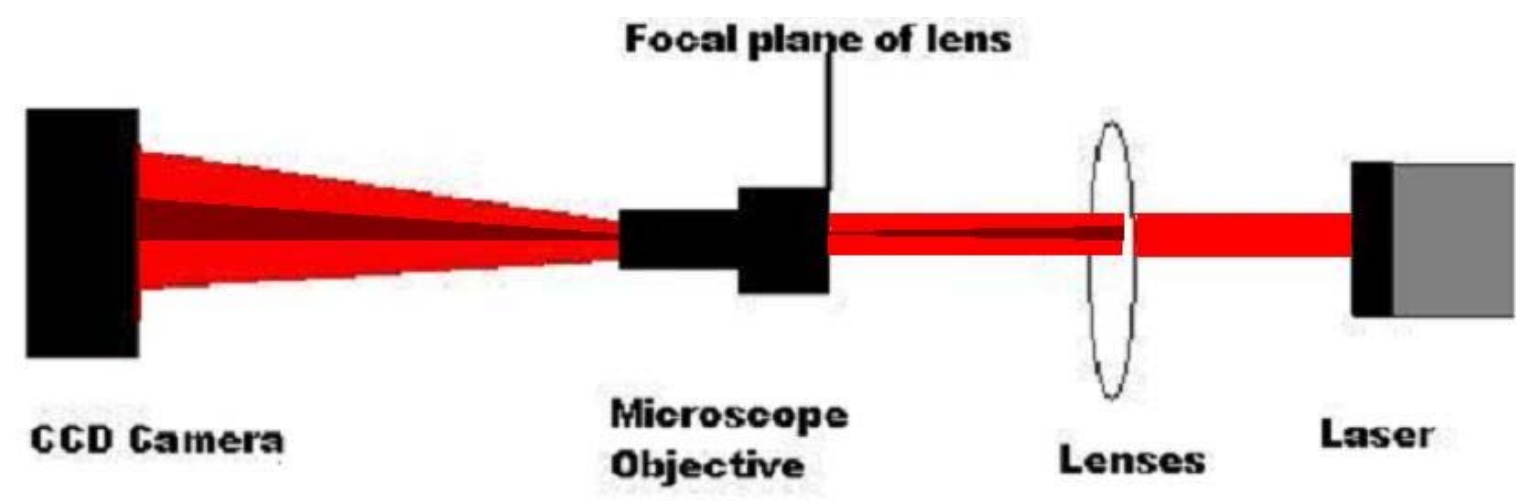

Figure 61 Drawing of lens and laser size difference seen in the test setup

Figures 60 and 61 show the power measurement problem. The light red beam is from the laser while the dark red beam is the focused light from the lens. This poses the problem of separating out and measuring only the power incident on the lens. The diameter of the power meter sensor $(10 \mathrm{~mm})$ is too large to capture only the focused light or its projection through the objective - which is between 200-500um wide at a distance of $1.73 \mathrm{~m}$ from the objective. The objective used to magnify the DOE lens spot also took in this unfocused laser light and transferred it to the CCD camera. Taking a power reading under that condition causes an inflated measurement since some of the stray light is also incident on the power meter sensor. I used a physical aperture to limit the amount of stray, unfocused laser power that could strike the camera sensor. This aperture was placed approximately $0.076 \mathrm{~m}$ from the ND filter housing which was attached to the CCD camera. The aperture would not close tightly enough around the projected beam spot if it was placed too close to the DOE lens. The laser spot from the lens on the camera was observed through the Spricon software. The aperture was then reduced in size to a point just before it affected the spot intensity. This aperture size was well within the $10 \mathrm{~mm}$ diameter of the power meter sensor. It also has an outer case diameter of $3.18 \mathrm{~cm}$ which sufficiently blocks stray unfocused laser light from reaching the $8.8 \times 6.6 \mathrm{~mm}$ CCD sensor. 


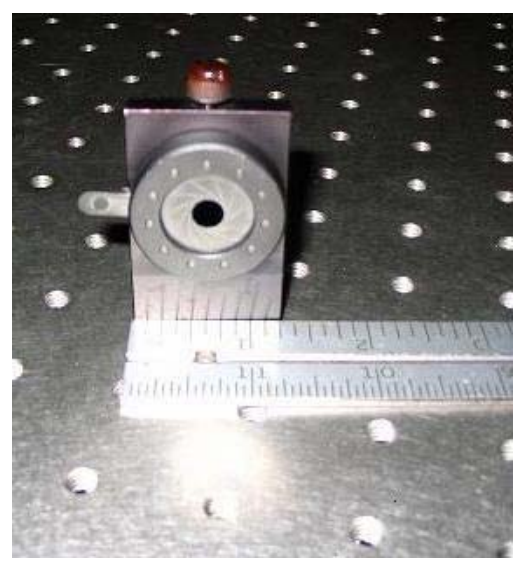

Figure 62 Aperture used to limit incident light from lens onto CCD camera

The Spiricon software allows input of measured incident light power. The Spiricon software then uses the measured intensity distribution and the measured incident power from the power meter to calculate the power density. A software aperture can then be made to measure the percent power contained within the software aperture at any place on the sensor. With this I have measured the power of the spot in the focal plane for the 8 on-axis lenses. I then took 125 samples of the laser at the position where the lenses were and used a software aperture that was $800 \mathrm{um}^{2}$ to measure the percent beam power incident on the lens. This is an approximation since I visually centered the laser on the lens by using an out of focus projection of the lens at the far end of the bench to align the center of the laser with the center of the lens. I also visually centered the software aperture on the measured laser beam to determine the percent of power incident on the lens. This method does not block out all of the stray beam light but is a good approximation to the lens spot power.

Taking the borofloat transmission into account gives a 1-3\% increase in efficiency for the lenses. An $80 \%$ borofloat efficiency would only give a 1-6\% increase in efficiency.

A power measurement was taken after the beam passed through the aperture. Loss in the objective was then accounted for. Another power measurement was taken at a point just before the laser was incident on the lens. The CCD camera was then placed at the location where the lens would be. The beam was sampled at this location. A square 800 um aperture (this is the size of the lens) was then created in the Spiricon program and centered on the 
sampled incident beam. This aperture could measure the approximate amount of light within the aperture and neglect any light power outside the aperture. This gives a relatively accurate measure of the light power incident on the lens assuming everything is centered.

\subsection{In-Plane Power Distribution and Beam Shape}

Figure 64 shows the C2R1 lens beam profile at its focal point. The graph in Figure 65 displays the power density profile at the focal point of lens $\mathrm{C} 2 \mathrm{R} 1$ produced when the lens is placed at 0.38 meters from the laser. This data was taken using an aperture to reduce the stray beam from reaching the camera. The measured power after the aperture was $0.9676 \mathrm{~mW}$ after accounting for the $6.986 \%$ microscope objective loss used to project the spot image onto the CCD camera. Power of the laser was $6.9 \mathrm{~mW}$. This was placed in the Spiricon software to get a peak power density of $917.2 \mathrm{~mW} / \mathrm{cm}^{2}$. The four smaller peaks were not used in the power profile and are less than ten percent of the peak height. The power density profile of the laser at 0.39 meters is overlaid onto the same graph for comparison in Figure 66.

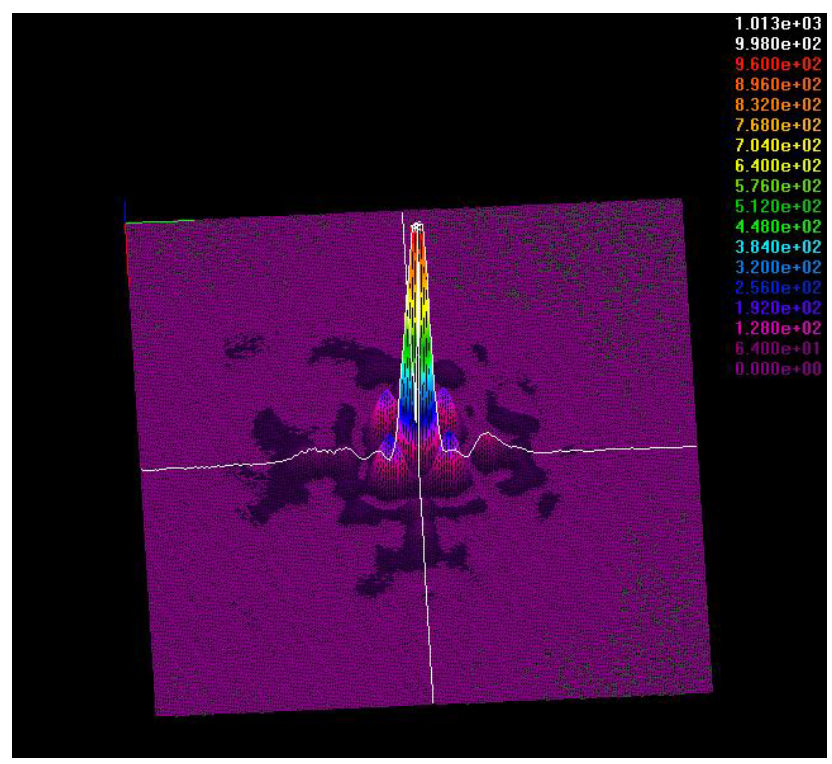

Figure 63 Lens focal point spot used to calculate power density 


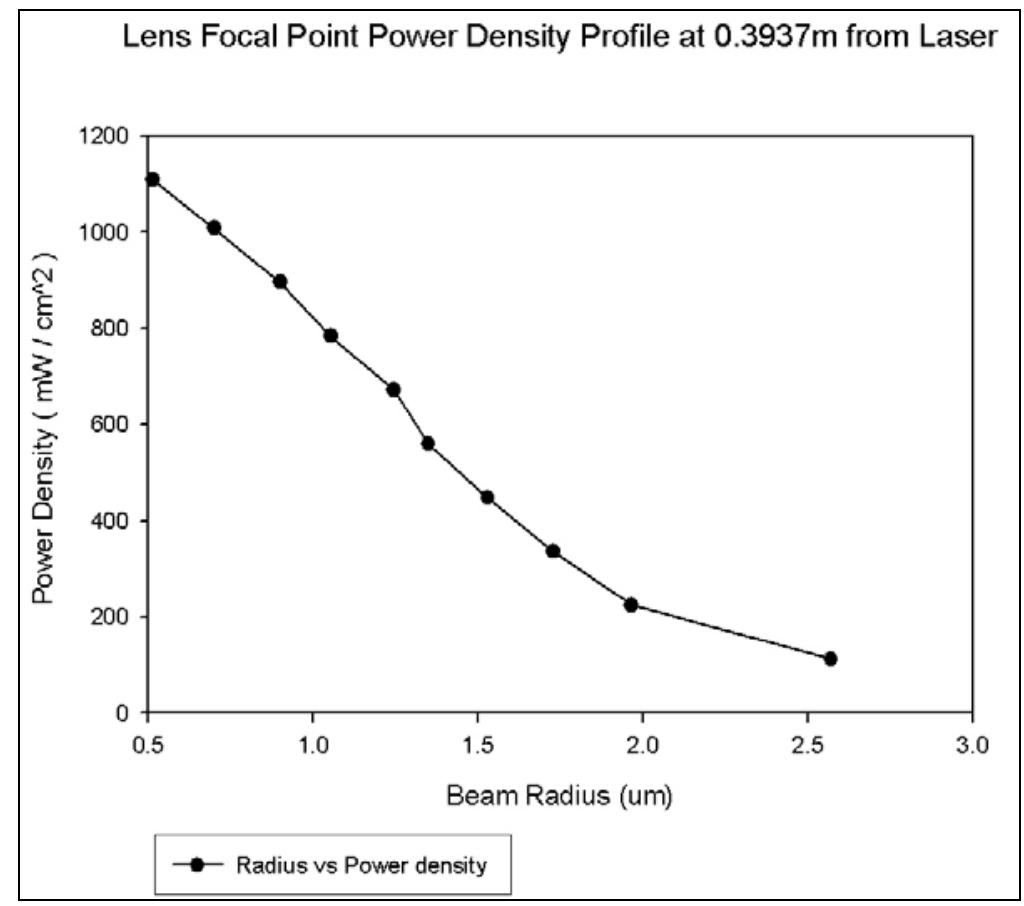

Figure 64 Lens focal point power density profile

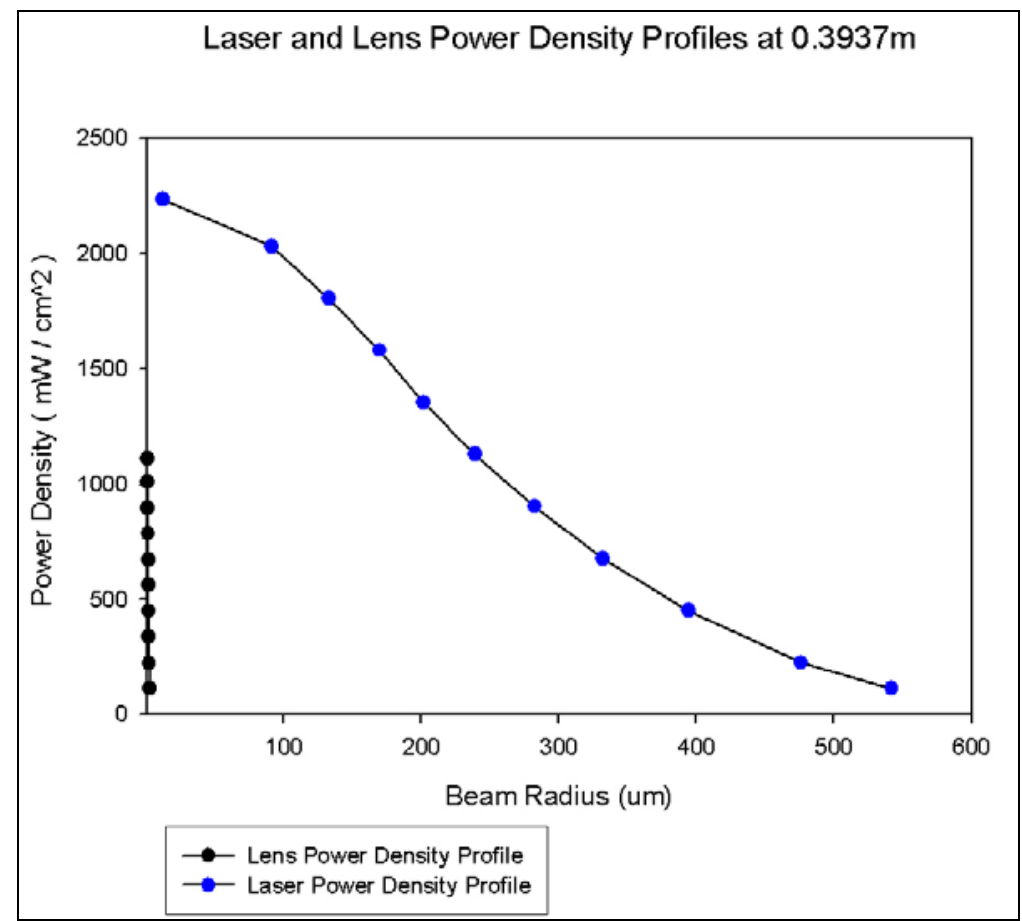

Figure 65 Comparison of laser and lens focal point power density profiles

Figure 65 is a comparison of the laser and lens power density profiles at a distance of 0.38 meters from the laser. The error for each point is less than $0.001 \mathrm{~mW} / \mathrm{cm}^{2}$ and so are not 
visible on the graph. The FWHM diameter of the laser was found to be 478 um while the FWHM diameter of the lens was 2.699um. This shows a 99.4\% reduction in FWHM diameter. The FWHM power density was $1152 \mathrm{~mW} / \mathrm{cm}^{2}$ for the laser and $560 \mathrm{~mW} / \mathrm{cm}^{2}$ for the lens. This gives a power density reduction of 51.3\% at FWHM. The peak power density was $2208 \mathrm{~mW} / \mathrm{cm}^{2}$ for the laser and $1119 \mathrm{~mW} / \mathrm{cm}^{2}$ for the lens. This is a $49.3 \%$ reduction in peak power density when using the lens. Power densities were obtained by measuring the incoming power from the laser or lens and entering that value into the Spiricon program. The measured laser diameter at 1.88 meters was $2.1 \mathrm{~mm}$ which is well within the $8.8 \times 6.6 \mathrm{~mm} \mathrm{CCD}$ sensor area. The aperture has an approximate outer case diameter of $3.18 \mathrm{~cm}$ which sufficiently blocks stray unfocused laser light from reaching the CCD sensor.

\begin{tabular}{|c|c|c|c|c|c|}
\hline Lens & $\begin{array}{l}\text { Average } \\
\text { Power of lens } \\
\text { Focal Point -- } \\
\text { using software } \\
\text { to measure } \\
\text { (W) }\end{array}$ & $\begin{array}{l}\text { Max Lens } \\
\text { Power } \\
\text { Through } \\
\text { Physical } \\
\text { Aperture } \\
\text { (W) }\end{array}$ & $\begin{array}{l}\text { Lens } \\
\text { (strict) } \\
\text { Efficiency }\end{array}$ & $\begin{array}{l}\text { Efficiency of } \\
\text { Borofloat } \\
\text { (Avg) }\end{array}$ & $\begin{array}{l}\text { Lens } \\
\text { Efficiency } \\
\text { (corrected } \\
\text { for borofloat } \\
\text { Tx } \\
\text { efficiency) }\end{array}$ \\
\hline $\mathrm{c} 1 \mathrm{r} 1$ & $1.035 \mathrm{E}-03$ & 1.362E-03 & $18 \%$ & $92 \%$ & $20 \%$ \\
\hline $\mathrm{c} 2 \mathrm{r} 1 \_1$ & $1.228 \mathrm{E}-04$ & 1.117E-03 & $2 \%$ & $92 \%$ & $2 \%$ \\
\hline $\mathrm{c} 2 \mathrm{r} 1 \_2$ & 3.898E-04 & 1.126E-03 & $7 \%$ & $92 \%$ & $8 \%$ \\
\hline c2r1_3 & 3.151E-04 & 1.102E-03 & $6 \%$ & $92 \%$ & $6 \%$ \\
\hline $\mathrm{c} 3 \mathrm{r} 1$ & 1.155E-03 & $1.541 \mathrm{E}-03$ & $20 \%$ & $92 \%$ & $23 \%$ \\
\hline c4r1_1 & $1.328 \mathrm{E}-04$ & 1.277E-03 & $2 \%$ & $92 \%$ & $3 \%$ \\
\hline$c 4 r 1 \_2$ & 4.039E-04 & 1.224E-03 & $7 \%$ & $92 \%$ & $8 \%$ \\
\hline c5r1 & 1.625E-03 & 1.812E-03 & $29 \%$ & $92 \%$ & $32 \%$ \\
\hline c6r1 & 1.334E-03 & 1.879E-03 & $23 \%$ & $92 \%$ & $26 \%$ \\
\hline $\mathrm{c} 7 \mathrm{r} 1$ & 1.565E-03 & 1.758E-03 & $28 \%$ & $92 \%$ & $31 \%$ \\
\hline c8r1 & 1.380E-03 & 1.840E-03 & $24 \%$ & $92 \%$ & $27 \%$ \\
\hline
\end{tabular}

Table 12 Lens Efficiency

The Spiricon software was used to determine if the aperture used caused a reduction in the projected peak of the lens spot. Lens spot data, consisting of 125 sample points, was collected while using the aperture and then without using the aperture. The Spiricon software was used to determine the peak power density for each of the two sets of data using the same input power. The data without the aperture in place had a peak power density of $407.8 \pm 0.06976$ $\mathrm{mW} / \mathrm{cm}^{2}$ while the data using the aperture showed $407.3 \pm 0 \mathrm{~mW} / \mathrm{cm}^{2}$. This shows that the 
aperture can be used to block unwanted incoming power without greatly affecting the peak of the lens spot.

\subsection{Visual Inspection of Lenses}

Visual inspection of the lenses was performed using a laser doppler vibrometer from Polytec that used an Olympus microscope. Visual inspection showed that some of the $633 \mathrm{~nm}$ lenses were not fully formed. This is likely due to the small feature size and conditions of the lab where they were made. The $1310 \mathrm{~nm}$ lenses had larger feature sizes yet still had some noticeable damage. Remaking the lenses in a clean room might improve the quality.
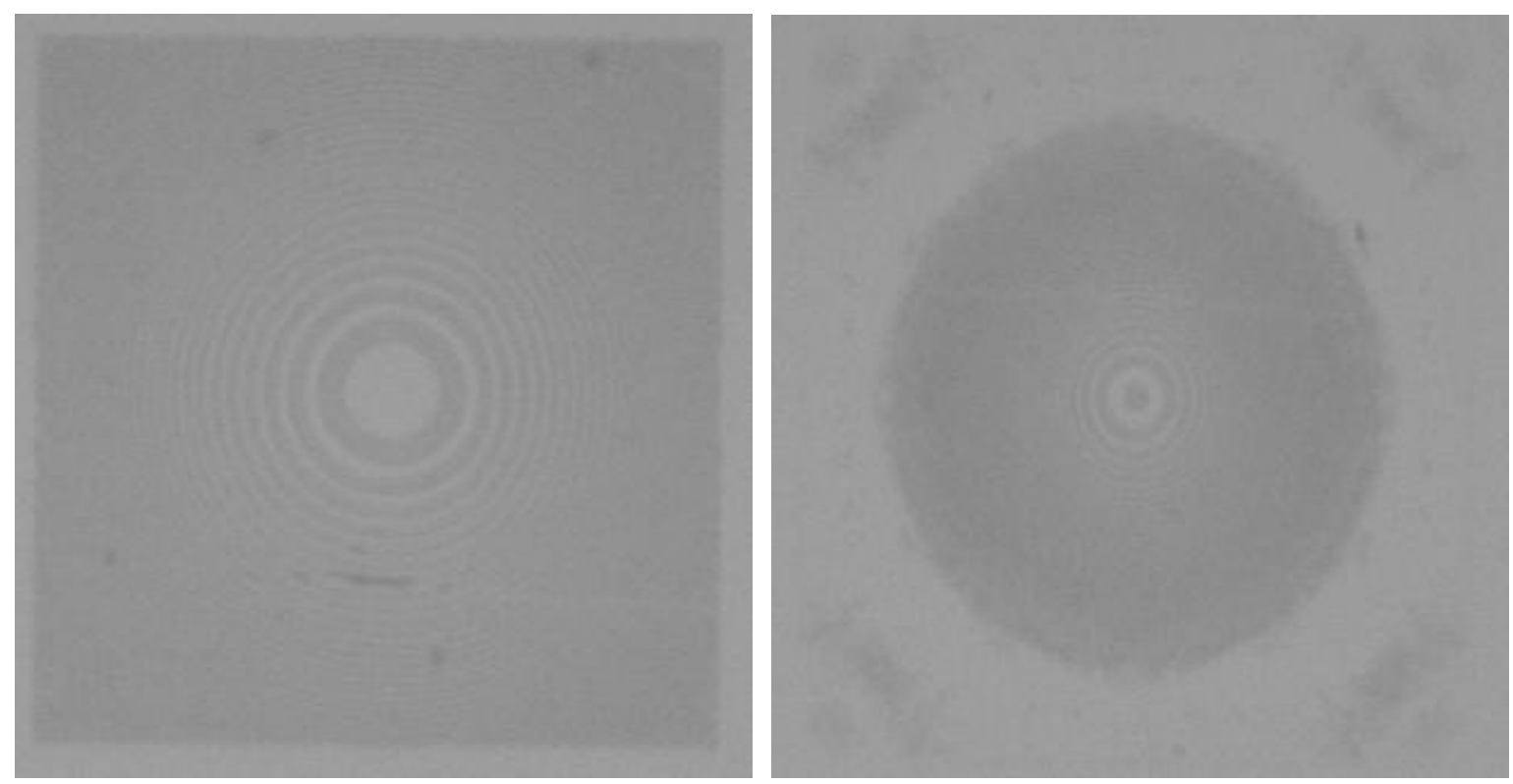

Figure 66 Lens C1R1 and C2R1 


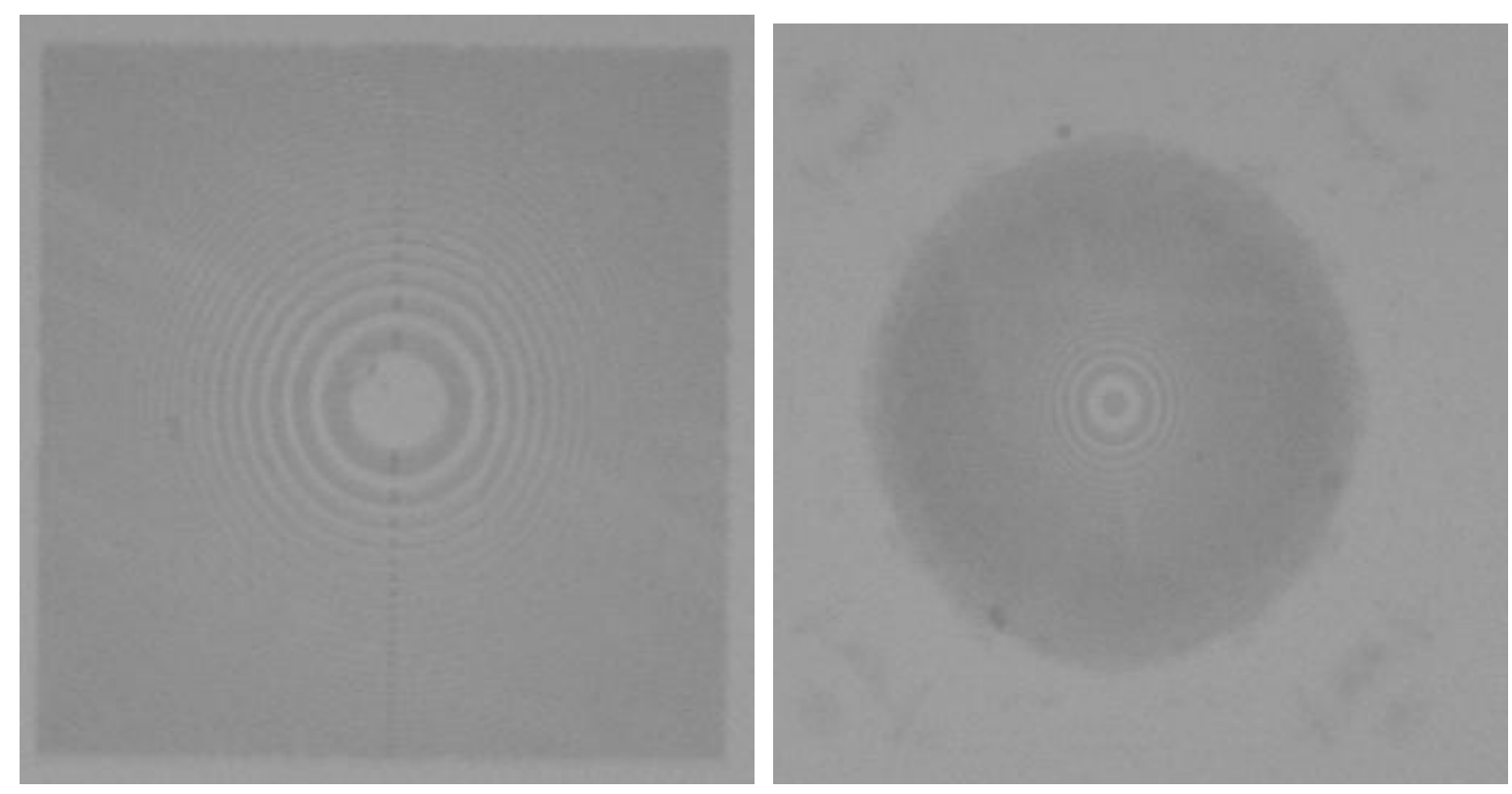

Figure 67 Lens C3R1 and C4R1

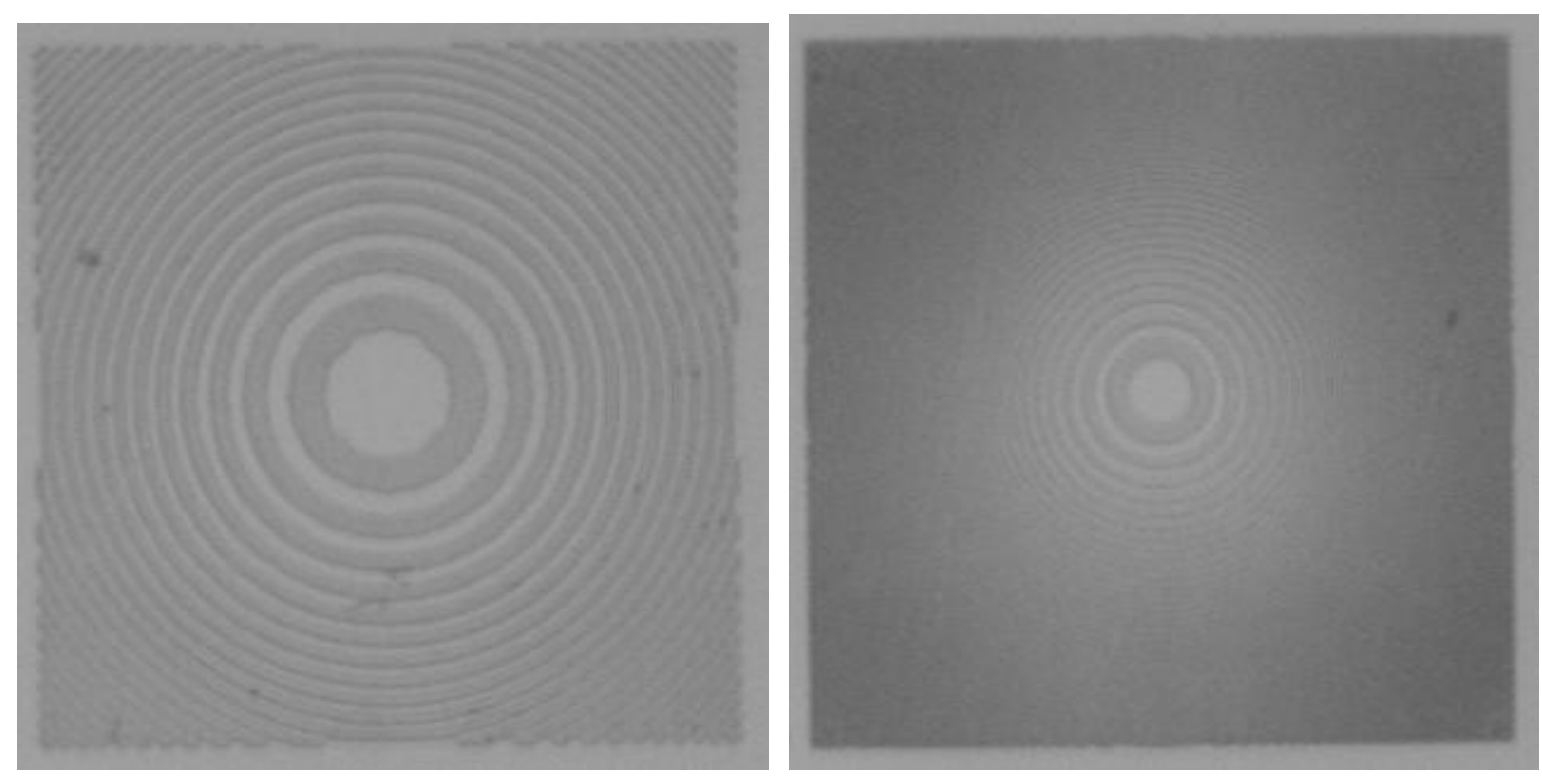

Figure 68 Lens C5R1 and C6R1 

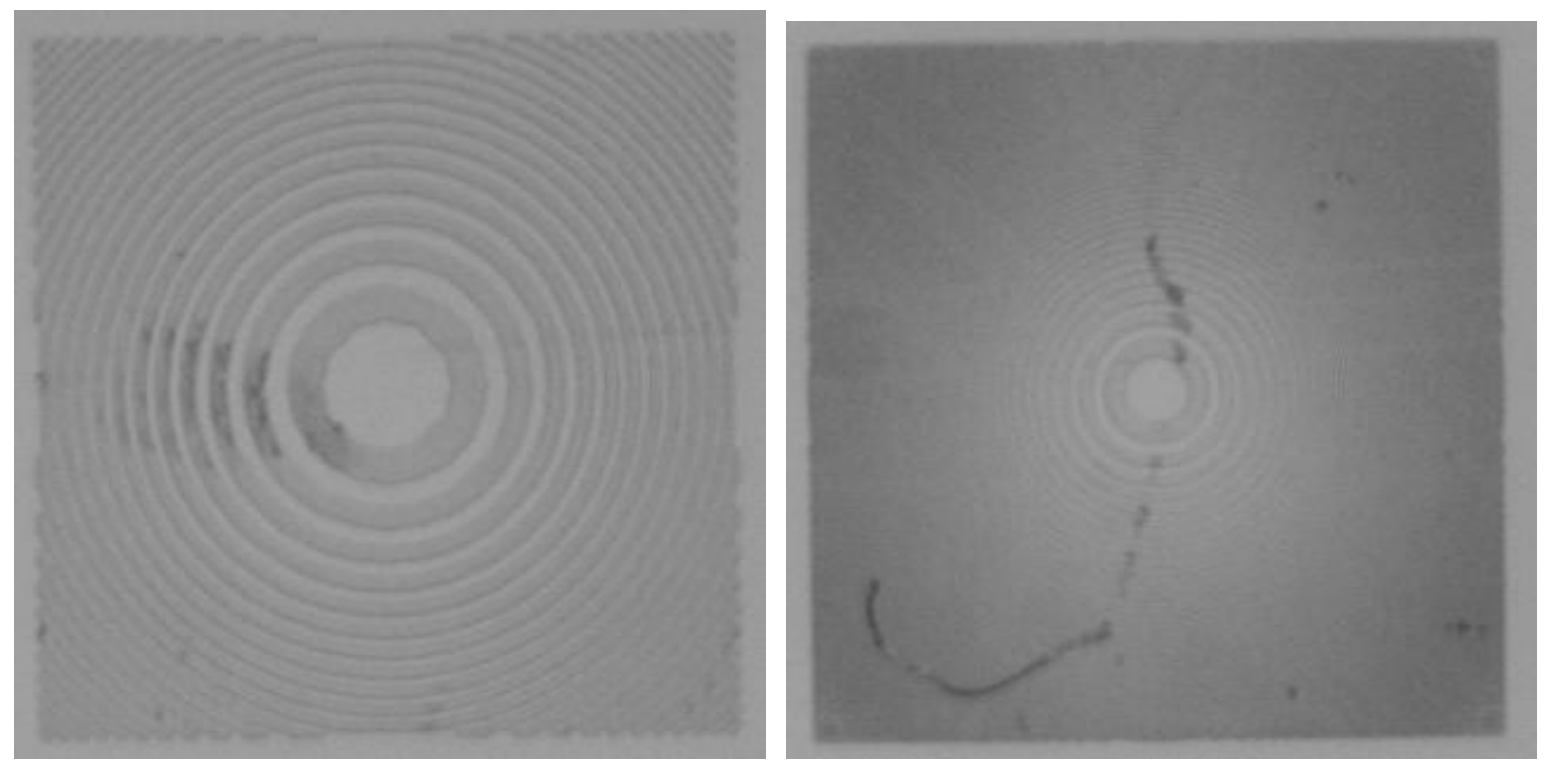

Figure 69 Lens C7R1 and C8R1

Lenses C2R1 and C4R1 demonstrated a lower signal to noise ratio and a great deal of distortion. This can be accounted for upon visual inspection since these lenses are not complete. Some of their diffraction ring pattern is missing which leads to noticeable distortion caused by light that is not canceled out as it should be from the diffraction pattern design.

\subsection{On Axis Lenses: Characterization Results}

All of the previous analysis were performed for only lens C2R1. The previous methods used to characterize lens $\mathrm{C} 2 \mathrm{R} 1$ are used here for each of the other lenses. Each page contains a 2-d and 3-d picture of the lens at its focal point along with a table of data relating to the picture above it. Each lens has 3 corresponding points of interest captured here: a point before the peak focal location, the peak focal location, and a point after the peak focal location. The before and after locations have a height roughly half of the lens' peak focal point and are arbitrarily used to define the focal range. The focal range could be extended depending on the application. The focal points were determined by placing the microscope objective close to the lens and moving it away until the focal point was reached. Micrometers shown in the picture below were used to measure the focal distances. 


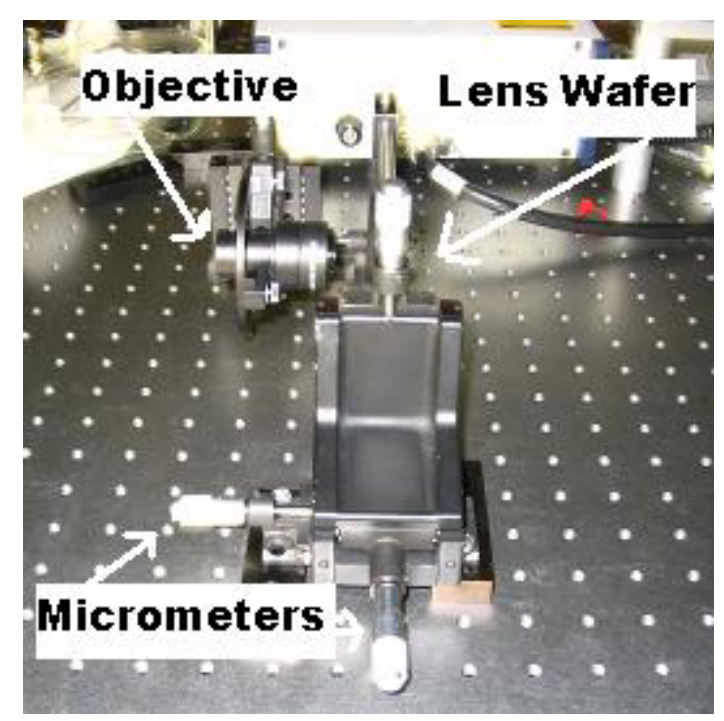

Figure 70 Test setup

The designation for each lens contains the column and row location (C3R1 - column 3 row

1). Two lenses (c2r1 and $\mathrm{c} 4 \mathrm{r} 1$ ) do not contain before and after peak data since it was difficult to discern these locations. Theses two lenses contain multiple peaks very close to one another and that data was recorded. The designation of each lens was verified by projecting the image of the column heading through the objective so that it could be read. All peaks were observed within a range of $2.54 \mathrm{~cm}$ from the $\mathrm{DOE}$ lens. 


\subsubsection{Lens Column 1 Row 1}

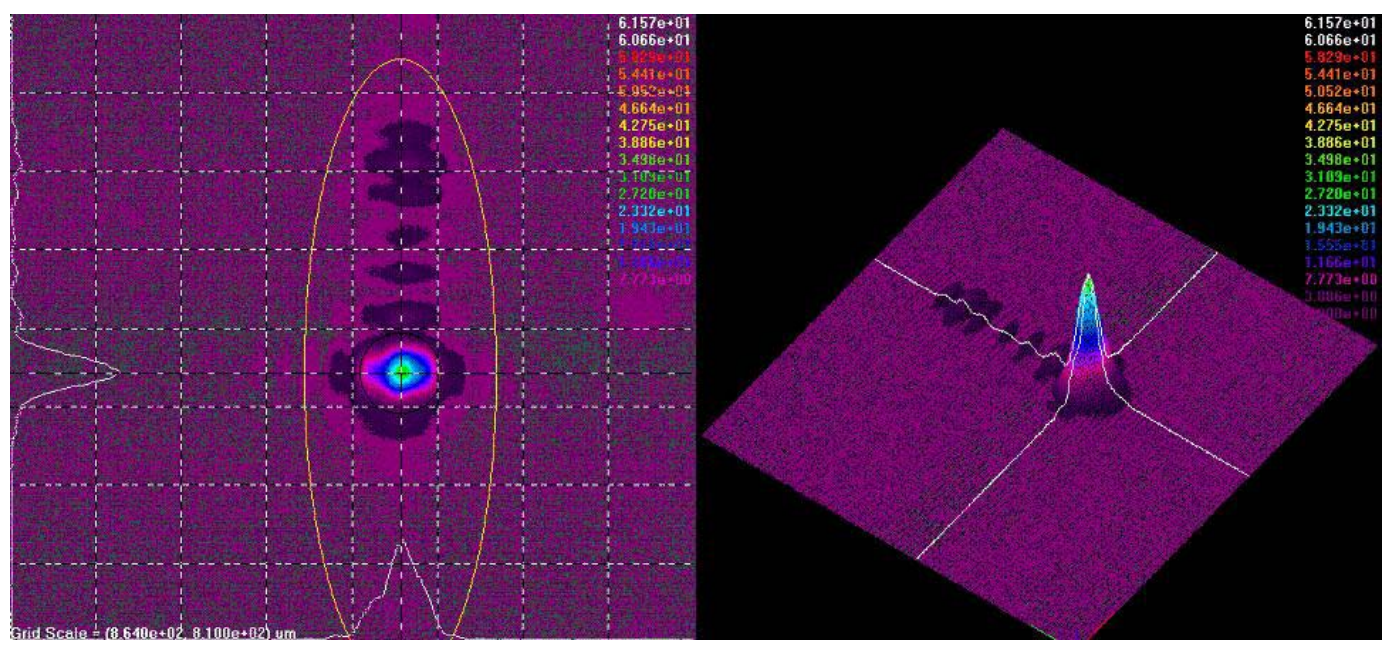

Figure 71 C1R1 Before

\begin{tabular}{|c|c|c|}
\hline \multicolumn{3}{|l|}{ Lens Designation C1R1 Before } \\
\hline Max Laser Power Through Aperture Using Objective (W) & $2.79 \mathrm{E}-04$ & \\
\hline Max Laser Power at Aperture Distance (W) & $6.87 \mathrm{E}-03$ & \\
\hline Laser Power Reduction Using Objective and Aperture (W) & $6.59 \mathrm{E}-03$ & $96 \%$ \\
\hline Magnification & 59.54 & \\
\hline Focal Disctance (mm) & 9.3472 & \\
\hline Focal range of lens (mm) & 0.22225 & \\
\hline & Avg & std \\
\hline Diameter FWHM (um) & $6.599 \mathrm{E}+00$ & $4.620 \mathrm{E}-02$ \\
\hline Diameter $13.5 \%$ (um) & $1.246 \mathrm{E}+01$ & $7.676 \mathrm{E}-02$ \\
\hline Max Lens Power Through Aperture (W) & $1.37 \mathrm{E}-03$ & -- \\
\hline Peak Power Density $\left(\mathrm{mW} / \mathrm{cm}^{\wedge} 2\right)$ & $5.729 \mathrm{E}+02$ & $2.384 \mathrm{E}+00$ \\
\hline Power Density FWHM $\left(\mathrm{mW} / \mathrm{cm}^{\wedge} 2\right)$ & $2.864 \mathrm{E}+02$ & $1.192 \mathrm{E}+00$ \\
\hline Power Density $13.5 \%\left(\mathrm{~mW} / \mathrm{cm}^{\wedge} 2\right)$ & $7.734 \mathrm{E}+01$ & $3.218 \mathrm{E}-01$ \\
\hline Objective Loss from 11_14_05 (W) & $7.28 \%$ & $0.86 \%$ \\
\hline Nearest Peak Power Density (W) & $5.992 \mathrm{E}+01$ & $8.153 \mathrm{E}-01$ \\
\hline SNR (based on power density) & $9.561 \mathrm{E}+00$ & $2.924 \mathrm{E}+00$ \\
\hline
\end{tabular}

Table 13 C1R1 Before 


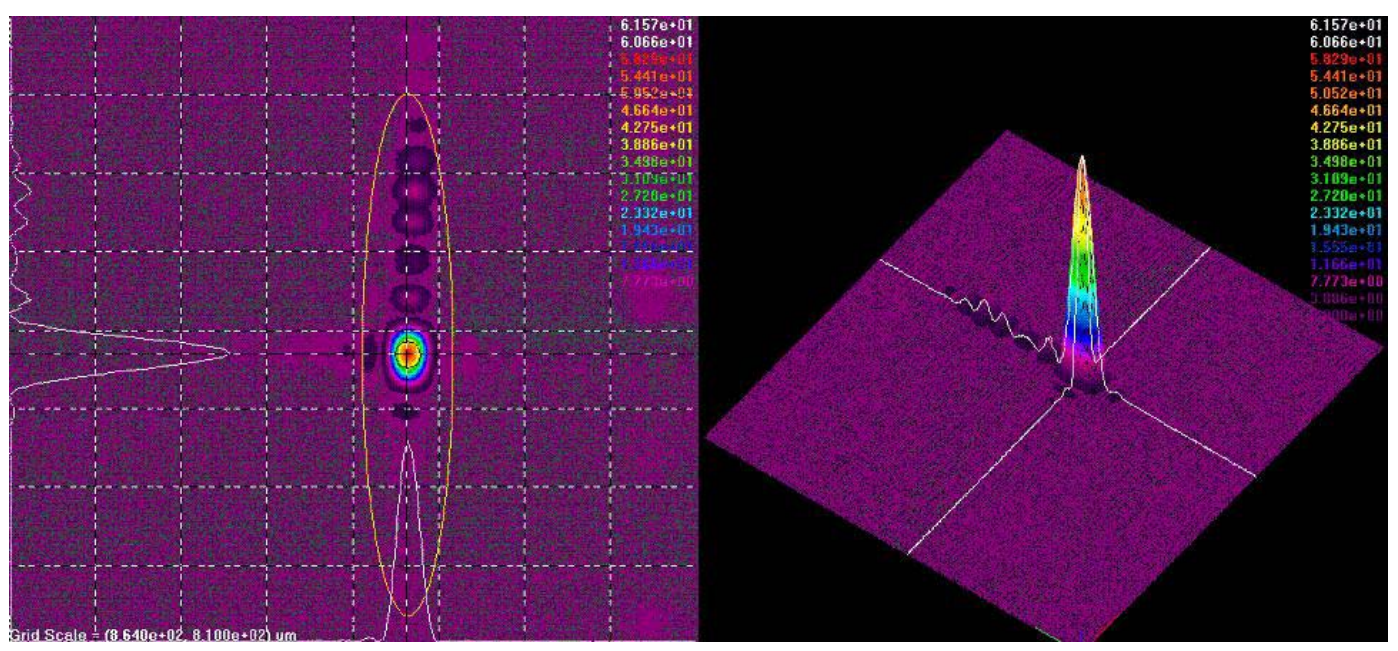

Figure 72 C1R1 Peak

\begin{tabular}{|c|c|c|}
\hline Max Laser Power Through Aperture Using Objective (W) & $2.79 E-04$ & \\
\hline Max Laser Power at Aperture Distance (W) & $6.87 E-03$ & \\
\hline Laser Power Reduction Using Objective and Aperture (W) & $6.59 \mathrm{E}-03$ & $96 \%$ \\
\hline Magnification & 59.54 & \\
\hline Focal Disctance (mm) & 9.45515 & \\
\hline Focal range of lens (mm) & 0.22225 & \\
\hline & Avg & std \\
\hline Diameter FWHM (um) & $5.299 \mathrm{E}+00$ & $9.007 \mathrm{E}-02$ \\
\hline Diameter $13.5 \%$ (um) & $8.450 \mathrm{E}+00$ & $6.683 E-02$ \\
\hline Max Lens Power Through Aperture (W) & $1.36 \mathrm{E}-03$ & \\
\hline Peak Power Density $\left(\mathrm{mW} / \mathrm{cm}^{\wedge} 2\right)$ & $1.003 \mathrm{E}+04$ & $1.807 \mathrm{E}+01$ \\
\hline Power Density FWHM $\left(\mathrm{mW} / \mathrm{cm}^{\wedge} 2\right)$ & $5.014 \mathrm{E}+03$ & $9.033 E+00$ \\
\hline Power Density $13.5 \%\left(\mathrm{~mW} / \mathrm{cm}^{\wedge} 2\right)$ & $1.354 \mathrm{E}+03$ & $2.439 \mathrm{E}+00$ \\
\hline Objective Loss from 11_14_05 (W) & $7.28 \%$ & $0.86 \%$ \\
\hline Nearest Peak Power Density (W) & $1.048 \mathrm{E}+03$ & $1.092 \mathrm{E}+01$ \\
\hline SNR (based on power density) & $9.566 \mathrm{E}+00$ & $1.654 \mathrm{E}+00$ \\
\hline Efficiency & $20 \%$ & \\
\hline
\end{tabular}

Table 14 C1R1 Peak 


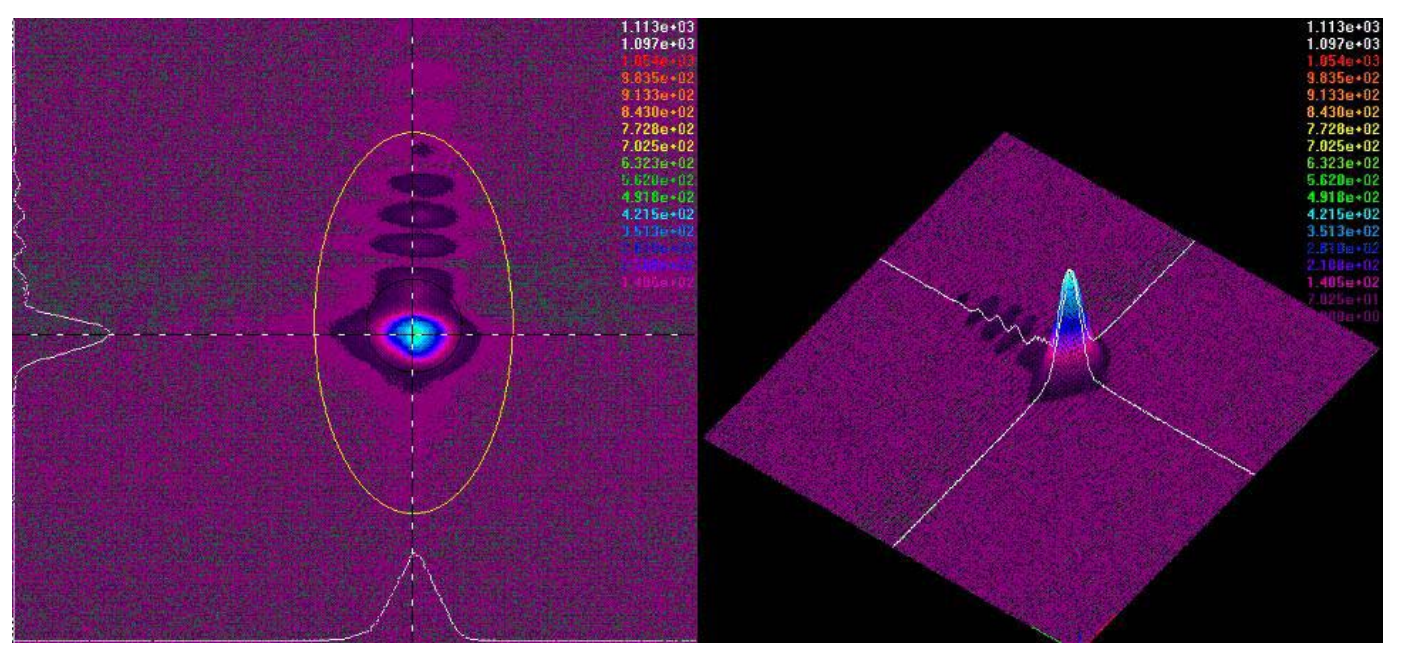

Figure 73 C1R1 After

\begin{tabular}{|l|r|r|}
\hline Max Laser Power Through Aperture Using Objective (W) & $2.79 \mathrm{E}-04$ & \\
\hline Max Laser Power at Aperture Distance (W) & $6.87 \mathrm{E}-03$ & \\
\hline Laser Power Reduction Using Objective and Aperture (W) & $6.59 \mathrm{E}-03$ & $96 \%$ \\
\hline \multicolumn{1}{|l|}{} & 59.54 & \\
\hline Magnification & 9.56945 & \\
\hline Focal Disctance (mm) & 0.22225 & \\
\hline Focal range of lens (mm) & Avg & std \\
\hline \multicolumn{1}{|l|}{} & $7.595 \mathrm{E}+00$ & $0.000 \mathrm{E}+00$ \\
\hline Diameter FWHM (um) & $1.346 \mathrm{E}+01$ & $1.876 \mathrm{E}-01$ \\
\hline Diameter 13.5\% (um) & $1.37 \mathrm{E}-03$ & \\
\hline Max Lens Power Through Aperture (W) & $5.153 \mathrm{E}+02$ & $1.788 \mathrm{E}+00$ \\
\hline Peak Power Density (mW/cm^2) & $2.576 \mathrm{E}+02$ & $8.942 \mathrm{E}-01$ \\
\hline Power Density FWHM (mW/cm^2) & $6.956 \mathrm{E}+01$ & $2.414 \mathrm{E}-01$ \\
\hline Power Density 13.5\% (mW/cm^2) & & \\
\hline Objective Loss from 11_14_05 (W) & $1.941 \mathrm{E}+02$ & $5.498 \mathrm{E}+00$ \\
\hline Nearest Peak Power Density (W) & $2.655 \mathrm{E}+00$ & $3.253 \mathrm{E}-01$ \\
\hline SNR (based on power density) & $7.28 \%$ & $0.86 \%$ \\
\hline
\end{tabular}

Table 15 C1R1 After 


\subsubsection{Lens Column 2 Row 1}

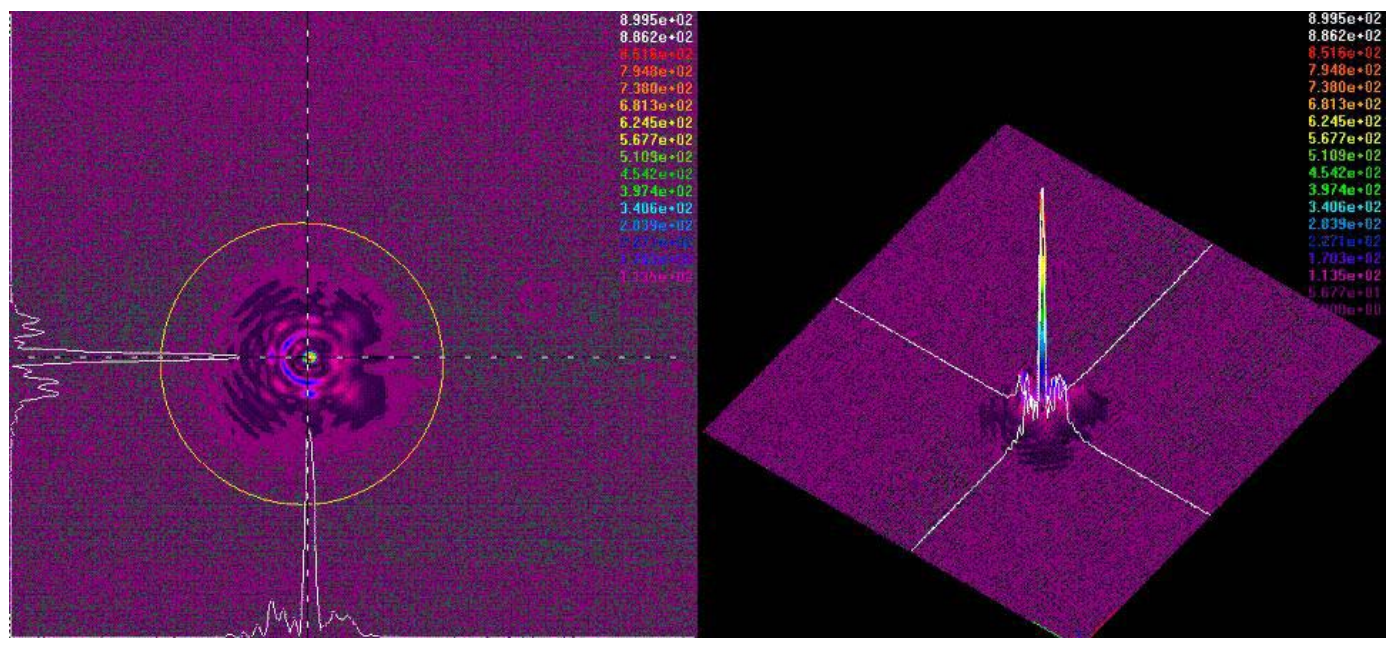

Figure 74 C2R1 Peak 1

\begin{tabular}{|c|c|c|}
\hline Max Laser Power Through Aperture Using Objective (W) & $2.79 \mathrm{E}-04$ & \\
\hline Max Laser Power at Aperture Distance (W) & $6.87 \mathrm{E}-03$ & \\
\hline Laser Power Reduction Using Objective and Aperture (W) & $6.59 \mathrm{E}-03$ & $96 \%$ \\
\hline Magnification & 59.54 & \\
\hline Focal Disctance (mm) & 12.954 & \\
\hline Focal range of lens $(\mathrm{mm})$ & -- & \\
\hline & Avg & std \\
\hline Diameter FWHM (um) & $1.799 \mathrm{E}+00$ & $8.393 E-02$ \\
\hline Diameter $13.5 \%$ (um) & $3.018 \mathrm{E}+00$ & $2.481 E-01$ \\
\hline Max Lens Power Through Aperture (W) & $1.12 \mathrm{E}-03$ & -- \\
\hline Peak Power Density $\left(\mathrm{mW} / \mathrm{cm}^{\wedge} 2\right)$ & $9.680 \mathrm{E}+02$ & $1.252 \mathrm{E}-01$ \\
\hline Power Density FWHM $\left(\mathrm{mW} / \mathrm{cm}^{\wedge} 2\right)$ & $4.840 \mathrm{E}+02$ & $6.260 \mathrm{E}-02$ \\
\hline Power Density $13.5 \%\left(\mathrm{~mW} / \mathrm{cm}^{\wedge} 2\right)$ & $1.307 \mathrm{E}+02$ & $1.690 \mathrm{E}-02$ \\
\hline Objective Loss from 11_14_05 (W) & $7.28 \%$ & $0.86 \%$ \\
\hline Nearest Peak Power Density (W) & $2.200 \mathrm{E}+02$ & $1.748 \mathrm{E}+00$ \\
\hline SNR (based on power density) & $4.399 \mathrm{E}+00$ & $7.164 \mathrm{E}-02$ \\
\hline Efficiency & $2 \%$ & \\
\hline
\end{tabular}

Table 16 C2R1 Peak 1 


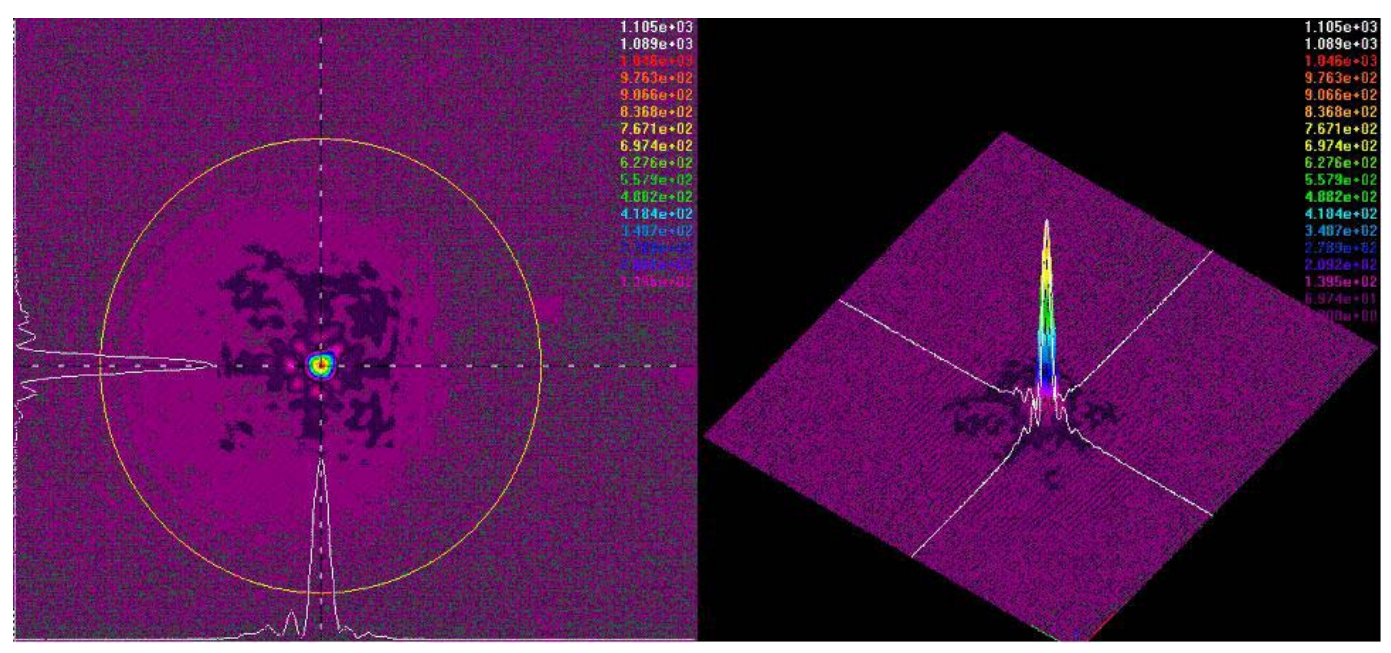

Figure 75 C2R1 Peak 2

\begin{tabular}{|c|c|c|}
\hline \multicolumn{3}{|l|}{ Lens Designation C2R1 Peak 2} \\
\hline Max Laser Power Through Aperture Using Objective (W) & $2.79 \mathrm{E}-04$ & \\
\hline Max Laser Power at Aperture Distance (W) & $6.87 E-03$ & \\
\hline Laser Power Reduction Using Objective and Aperture (W) & $6.59 E-03$ & 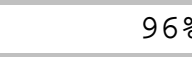 \\
\hline Magnification & 59.54 & \\
\hline Focal Disctance (mm) & 13.0683 & \\
\hline Focal range of lens $(\mathrm{mm})$ & -- & \\
\hline & Avg & std \\
\hline Diameter FWHM (um) & $3.211 \mathrm{E}+00$ & $6.070 \mathrm{E}-02$ \\
\hline Diameter $13.5 \%$ (um) & $5.568 \mathrm{E}+00$ & $7.511 \mathrm{E}-02$ \\
\hline Max Lens Power Through Aperture (W) & $1.13 \mathrm{E}-03$ & \\
\hline Peak Power Density $\left(\mathrm{mW} / \mathrm{cm}^{\wedge} 2\right)$ & $1.049 \mathrm{E}+03$ & $3.298 \mathrm{E}+00$ \\
\hline Power Density FWHM $\left(\mathrm{mW} / \mathrm{cm}^{\wedge} 2\right)$ & $5.247 \mathrm{E}+02$ & $1.649 \mathrm{E}+0 \mathrm{C}$ \\
\hline Power Density $13.5 \%\left(\mathrm{~mW} / \mathrm{cm}^{\wedge} 2\right)$ & $1.417 \mathrm{E}+02$ & $4.452 \mathrm{E}-01$ \\
\hline Objective Loss from 11_14_05 (W) & $7.28 \%$ & $0.86 \%$ \\
\hline Nearest Peak Power Density (W) & $1.744 \mathrm{E}+02$ & $1.845 \mathrm{E}+0 \mathrm{C}$ \\
\hline SNR (based on power density) & $6.016 \mathrm{E}+00$ & $1.787 \mathrm{E}+0 \mathrm{C}$ \\
\hline Efficiency & $8 \%$ & \\
\hline
\end{tabular}

Table 17 C2R1 Peak 2 


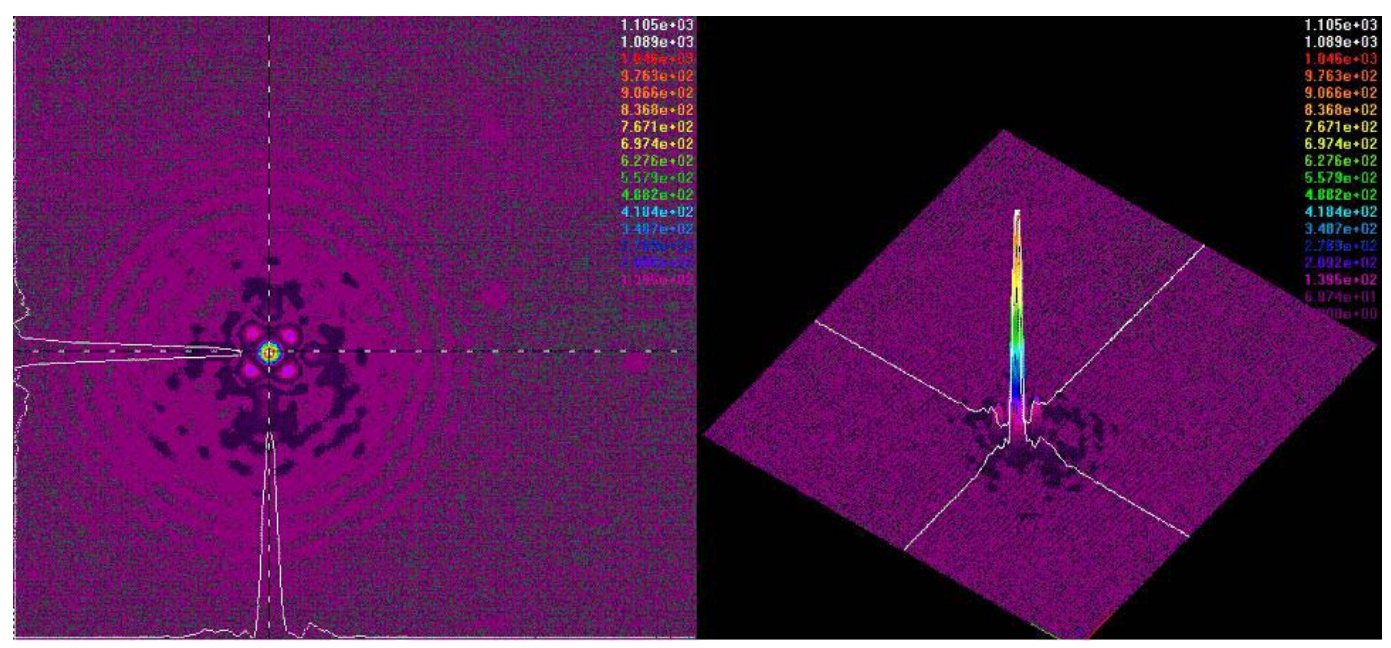

Figure 76 C2R1 Peak 3

\begin{tabular}{|c|c|c|}
\hline \multicolumn{3}{|l|}{ Lens Designation C2R1 Peak 3} \\
\hline Max Laser Power Through Aperture Using Objective (W) & $2.79 E-04$ & \\
\hline Max Laser Power at Aperture Distance (W) & $6.87 \mathrm{E}-03$ & \\
\hline Laser Power Reduction Using Objective and Aperture (W) & $6.59 \mathrm{E}-03$ & $96 \%$ \\
\hline Magnification & 59.54 & \\
\hline Focal Disctance (mm) & 16.0528 & \\
\hline Focal range of lens $(\mathrm{mm})$ & -- & \\
\hline & Avg & std \\
\hline Diameter FWHM (um) & $2.817 \mathrm{E}+00$ & $5.831 \mathrm{E}-02$ \\
\hline Diameter $13.5 \%$ (um) & $4.246 \mathrm{E}+00$ & $5.841 \mathrm{E}-02$ \\
\hline Max Lens Power Through Aperture (W) & $1.10 \mathrm{E}-03$ & \\
\hline Peak Power Density $\left(\mathrm{mW} / \mathrm{cm}^{\wedge} 2\right)$ & $1.159 \mathrm{E}+03$ & $5.937 \mathrm{E}-02$ \\
\hline Power Density FWHM $\left(\mathrm{mW} / \mathrm{cm}^{\wedge} 2\right)$ & $5.793 \mathrm{E}+02$ & $2.968 \mathrm{E}-02$ \\
\hline Power Density $13.5 \%\left(\mathrm{~mW} / \mathrm{cm}^{\wedge} 2\right)$ & $1.564 \mathrm{E}+02$ & $8.015 E-03$ \\
\hline Objective Loss from 11_14_05 (W) & $7.28 \%$ & $0.86 \%$ \\
\hline Nearest Peak Power Density (W) & $2.062 \mathrm{E}+02$ & $2.431 \mathrm{E}+00$ \\
\hline SNR(based on power density) & $5.619 \mathrm{E}+00$ & $2.442 \mathrm{E}-02$ \\
\hline Efficiency & $6 \%$ & \\
\hline
\end{tabular}

Table 18 C2R1 Peak 3 


\subsubsection{Lens Column 3 Row 1}

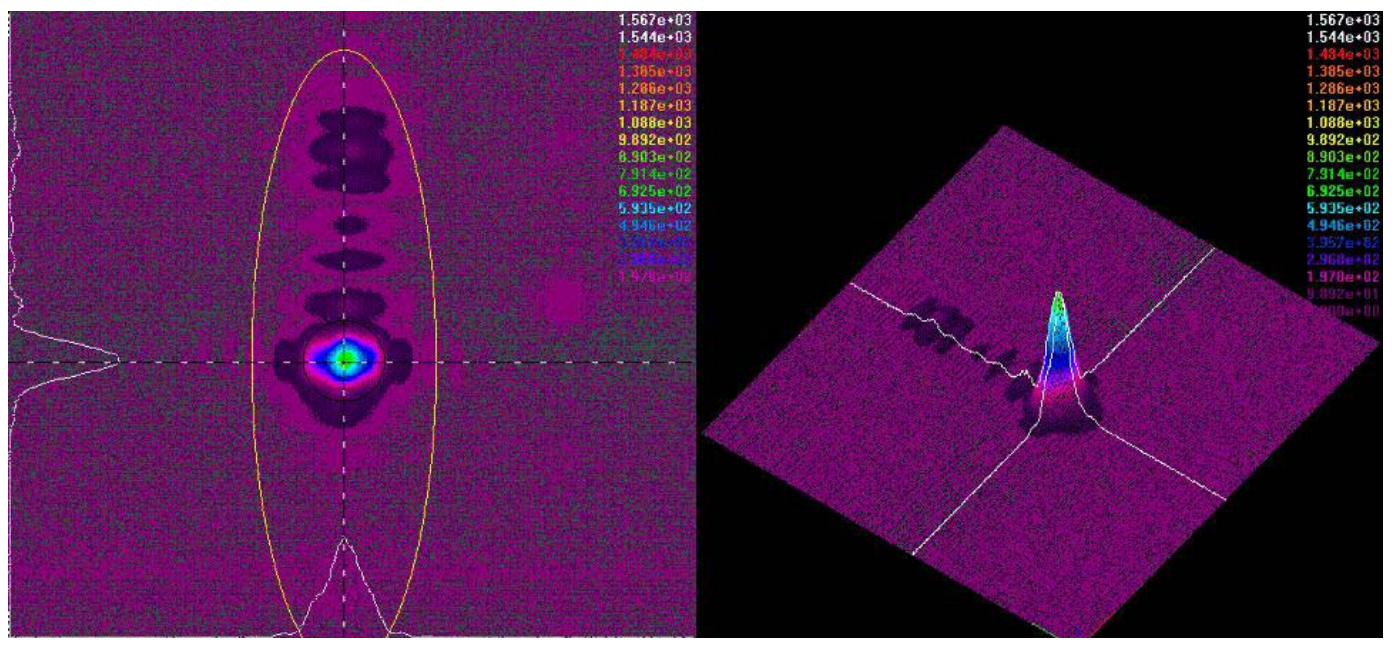

Figure 77 C3R1 Before

\begin{tabular}{|c|c|c|}
\hline \multicolumn{3}{|l|}{ Lens Designation C3R1 Before } \\
\hline Max Laser Power Through Aperture Using Objective (W) & $2.79 \mathrm{E}-04$ & \\
\hline Max Laser Power at Aperture Distance (W) & $6.87 \mathrm{E}-03$ & \\
\hline Laser Power Reduction Using Objective and Aperture (W) & $6.59 \mathrm{E}-03$ & $96 \%$ \\
\hline Magnification & 59.54 & \\
\hline Focal Disctance (mm) & 9.3472 & \\
\hline Focal range of lens (mm) & 0.2159 & \\
\hline & Avg & std \\
\hline Diameter FWHM (um) & $7.027 \mathrm{E}+00$ & $5.934 \mathrm{E}-02$ \\
\hline Diameter $13.5 \%$ (um) & $1.246 \mathrm{E}+01$ & $6.713 \mathrm{E}-02$ \\
\hline Max Lens Power Through Aperture (W) & $1.57 \mathrm{E}-03$ & -- \\
\hline Peak Power Density $\left(\mathrm{mW} / \mathrm{cm}^{\wedge} 2\right)$ & $8.263 E+02$ & $2.784 \mathrm{E}+00$ \\
\hline Power Density FWHM $\left(\mathrm{mW} / \mathrm{cm}^{\wedge} 2\right)$ & $4.131 E+02$ & $1.392 \mathrm{E}+00$ \\
\hline Power Density $13.5 \%\left(\mathrm{~mW} / \mathrm{cm}^{\wedge} 2\right)$ & $1.115 \mathrm{E}+02$ & $3.758 \mathrm{E}-01$ \\
\hline Objective Loss from 11_14_05 (W) & $7.28 \%$ & $0.86 \%$ \\
\hline Nearest Peak Power Density (W) & $1.074 \mathrm{E}+02$ & $1.414 \mathrm{E}+00$ \\
\hline SNR (based on power density) & $7.694 \mathrm{E}+00$ & $1.969 \mathrm{E}+00$ \\
\hline
\end{tabular}

Table 19 C3R1 Before 


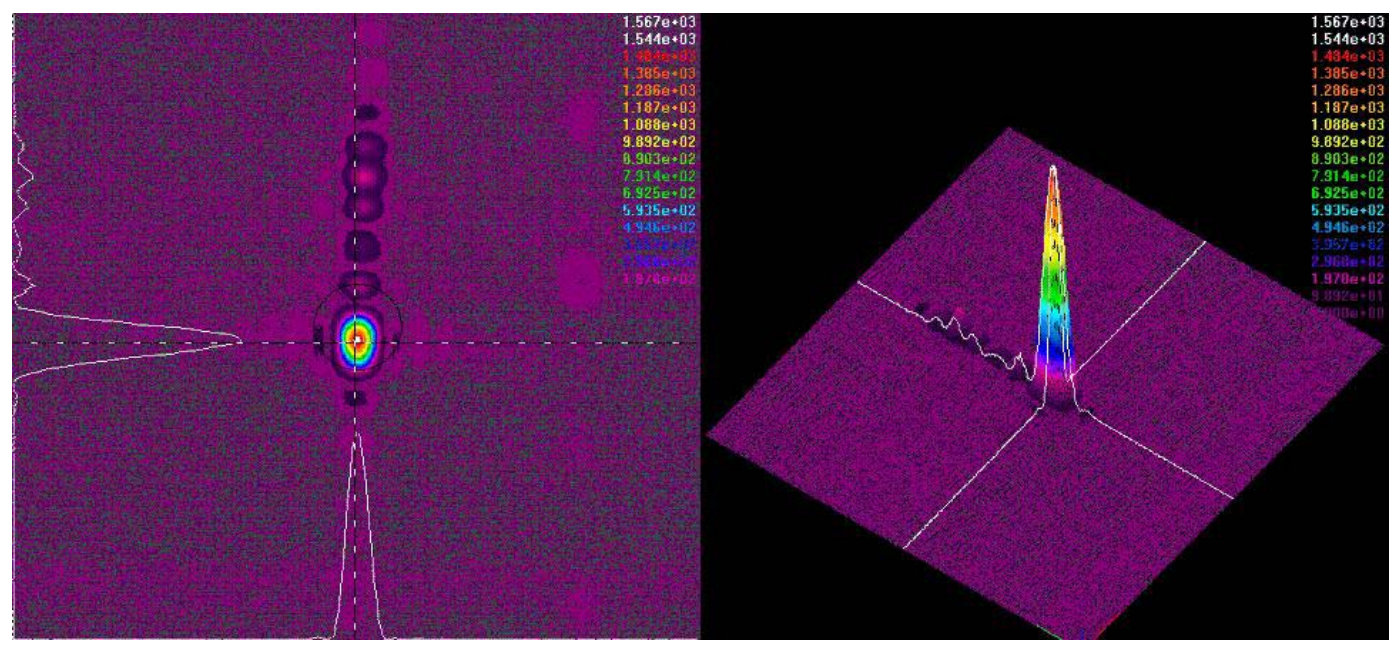

Figure 78 C3R1 Peak

\begin{tabular}{|c|c|c|}
\hline Max Laser Power Through Aperture Using Objective (W) & $2.79 \mathrm{E}-04$ & \\
\hline Max Laser Power at Aperture Distance (W) & $6.87 \mathrm{E}-03$ & \\
\hline Laser Power Reduction Using Objective and Aperture (W) & $6.59 \mathrm{E}-03$ & $96 \%$ \\
\hline Magnification & 59.54 & \\
\hline Focal Disctance (mm) & 9.45515 & \\
\hline Focal range of lens $(\mathrm{mm})$ & 0.2159 & \\
\hline & Avg & std \\
\hline Diameter FWHM (um) & $5.348 \mathrm{E}+00$ & $4.933 E-02$ \\
\hline Diameter $13.5 \%$ (um) & $8.446 \mathrm{E}+00$ & $6.999 \mathrm{E}-02$ \\
\hline Max Lens Power Through Aperture (W) & $1.54 \mathrm{E}-03$ & \\
\hline Peak Power Density $\left(\mathrm{mW} / \mathrm{cm}^{\wedge} 2\right)$ & $1.212 \mathrm{E}+03$ & $1.040 \mathrm{E}-01$ \\
\hline Power Density FWHM $\left(\mathrm{mW} / \mathrm{cm}^{\wedge} 2\right)$ & $6.061 \mathrm{E}+02$ & $5.198 E-02$ \\
\hline Power Density $13.5 \%\left(\mathrm{~mW} / \mathrm{cm}^{\wedge} 2\right)$ & $1.637 \mathrm{E}+02$ & $1.403 E-02$ \\
\hline Objective Loss from 11_14_05 (W) & $7.28 \%$ & $0.86 \%$ \\
\hline Nearest Peak Power Density (W) & $1.397 \mathrm{E}+02$ & $1.608 \mathrm{E}+00$ \\
\hline SNR (based on power density) & $8.679 \mathrm{E}+00$ & $6.464 \mathrm{E}-02$ \\
\hline Efficiency & $23 \%$ & \\
\hline
\end{tabular}

Table 20 C3R1 Peak 


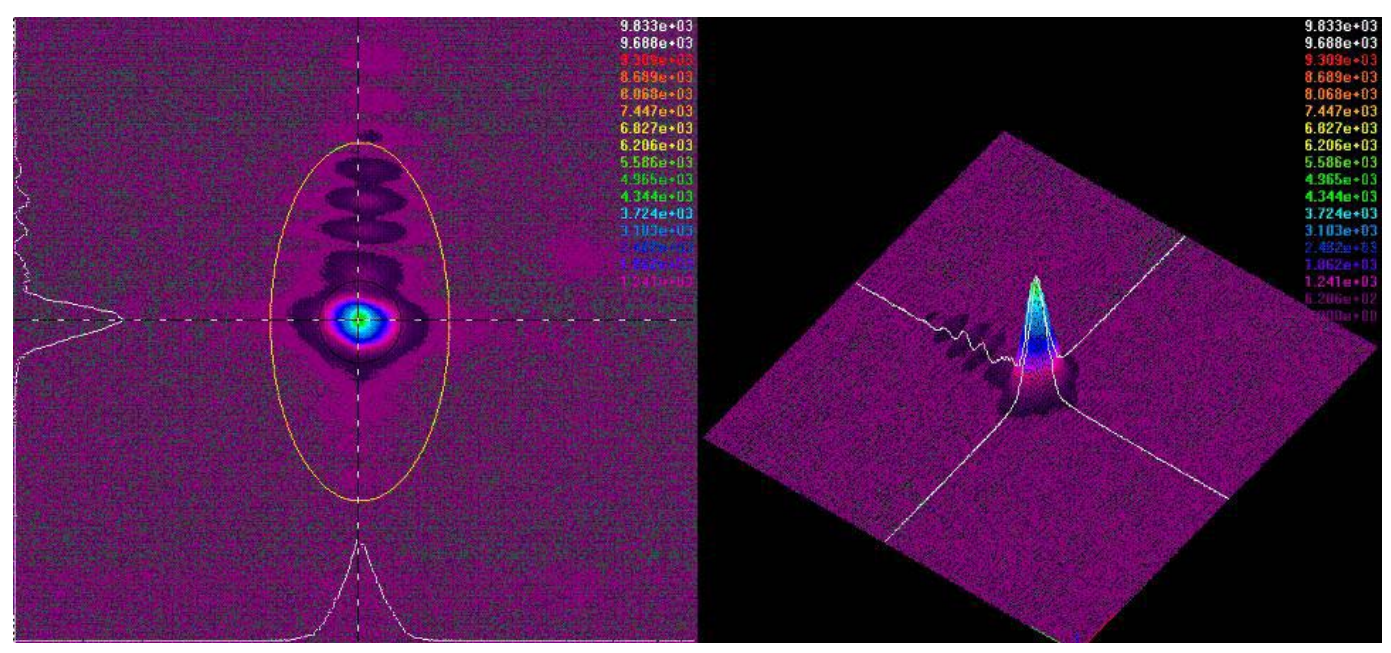

Figure 79 C3R1 After

\begin{tabular}{|c|c|c|}
\hline Max Laser Power Through Aperture Using Objective (W) & $2.79 \mathrm{E}-04$ & \\
\hline Max Laser Power at Aperture Distance (W) & $6.87 \mathrm{E}-03$ & \\
\hline Laser Power Reduction Using Objective and Aperture (W) & $6.59 \mathrm{E}-03$ & $96 \%$ \\
\hline Magnification & 59.54 & \\
\hline Focal Disctance (mm) & 9.5631 & \\
\hline Focal range of lens (mm) & 0.2159 & \\
\hline & Avg & std \\
\hline Diameter FWHM (um) & $6.648 \mathrm{E}+00$ & $6.364 \mathrm{E}-02$ \\
\hline Diameter $13.5 \%$ (um) & $1.307 \mathrm{E}+01$ & $9.807 \mathrm{E}-02$ \\
\hline Max Lens Power Through Aperture (W) & $1.54 \mathrm{E}-03$ & \\
\hline Peak Power Density $\left(\mathrm{mW} / \mathrm{cm}^{\wedge} 2\right)$ & $5.169 \mathrm{E}+03$ & $1.918 \mathrm{E}+01$ \\
\hline Power Density FWHM $\left(\mathrm{mW} / \mathrm{cm}^{\wedge} 2\right)$ & $2.584 \mathrm{E}+03$ & $9.591 \mathrm{E}+00$ \\
\hline Power Density $13.5 \%\left(\mathrm{~mW} / \mathrm{cm}^{\wedge} 2\right)$ & $6.978 \mathrm{E}+02$ & $2.590 \mathrm{E}+00$ \\
\hline Objective Loss from 11_14_05 (W) & $7.28 \%$ & $0.86 \%$ \\
\hline Nearest Peak Power Density (W) & $8.200 \mathrm{E}+02$ & $6.138 \mathrm{E}+01$ \\
\hline SNR (based on power density) & $6.304 \mathrm{E}+00$ & $3.125 \mathrm{E}-01$ \\
\hline
\end{tabular}

Table 21 C3R1 After 


\subsubsection{Lens Column 4 Row 1}

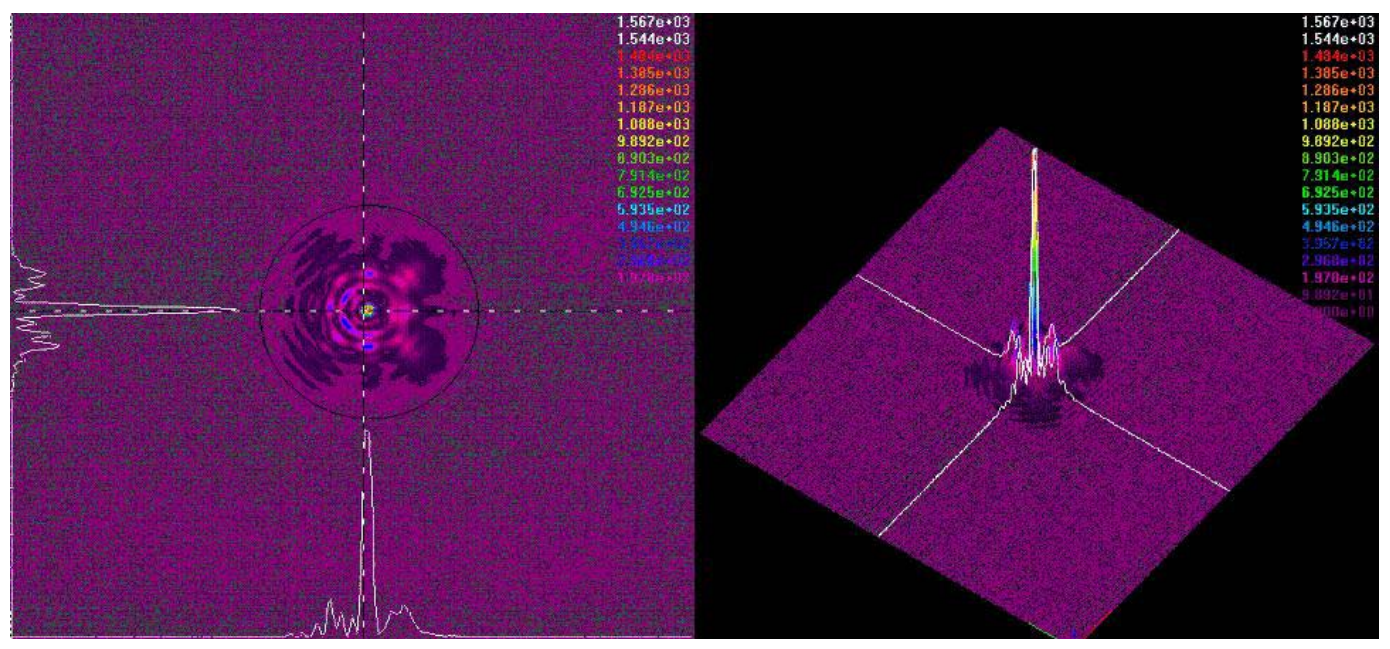

Figure 80 C4R1 Peak 1

\begin{tabular}{|c|c|c|}
\hline Max Laser Power Through Aperture Using Objective (W) & $2.79 E-04$ & \\
\hline Max Laser Power at Aperture Distance (W) & $6.87 \mathrm{E}-03$ & \\
\hline Laser Power Reduction Using Objective and Aperture (W) & $6.59 \mathrm{E}-03$ & $96 \%$ \\
\hline Magnification & 59.54 & \\
\hline Focal Disctance (mm) & 12.8524 & \\
\hline Focal range of lens $(\mathrm{mm})$ & -- & \\
\hline & Avg & std \\
\hline Diameter FWHM (um) & $1.841 \mathrm{E}+00$ & $4.805 \mathrm{E}-02$ \\
\hline Diameter $13.5 \%$ (um) & $2.686 \mathrm{E}+00$ & $5.799 \mathrm{E}-02$ \\
\hline Max Lens Power Through Aperture (W) & $1.28 \mathrm{E}-03$ & -- \\
\hline Peak Power Density $\left(\mathrm{mW} / \mathrm{cm}^{\wedge} 2\right)$ & $1.091 \mathrm{E}+03$ & $9.290 \mathrm{E}-02$ \\
\hline Power Density FWHM $\left(\mathrm{mW} / \mathrm{cm}^{\wedge} 2\right)$ & $5.455 \mathrm{E}+02$ & $4.645 \mathrm{E}-02$ \\
\hline Power Density $13.5 \%\left(\mathrm{~mW} / \mathrm{cm}^{\wedge} 2\right)$ & $1.473 \mathrm{E}+02$ & $1.254 \mathrm{E}-02$ \\
\hline Objective Loss from 11_14_05 (W) & $7.28 \%$ & $0.86 \%$ \\
\hline Nearest Peak Power Density (W) & $2.585 \mathrm{E}+02$ & $0.000 \mathrm{E}+00$ \\
\hline SNR (based on power density) & $4.220 \mathrm{E}+00$ & -- \\
\hline Efficiency & $3 \%$ & \\
\hline
\end{tabular}

Table 22 C4R1 Peak 1 


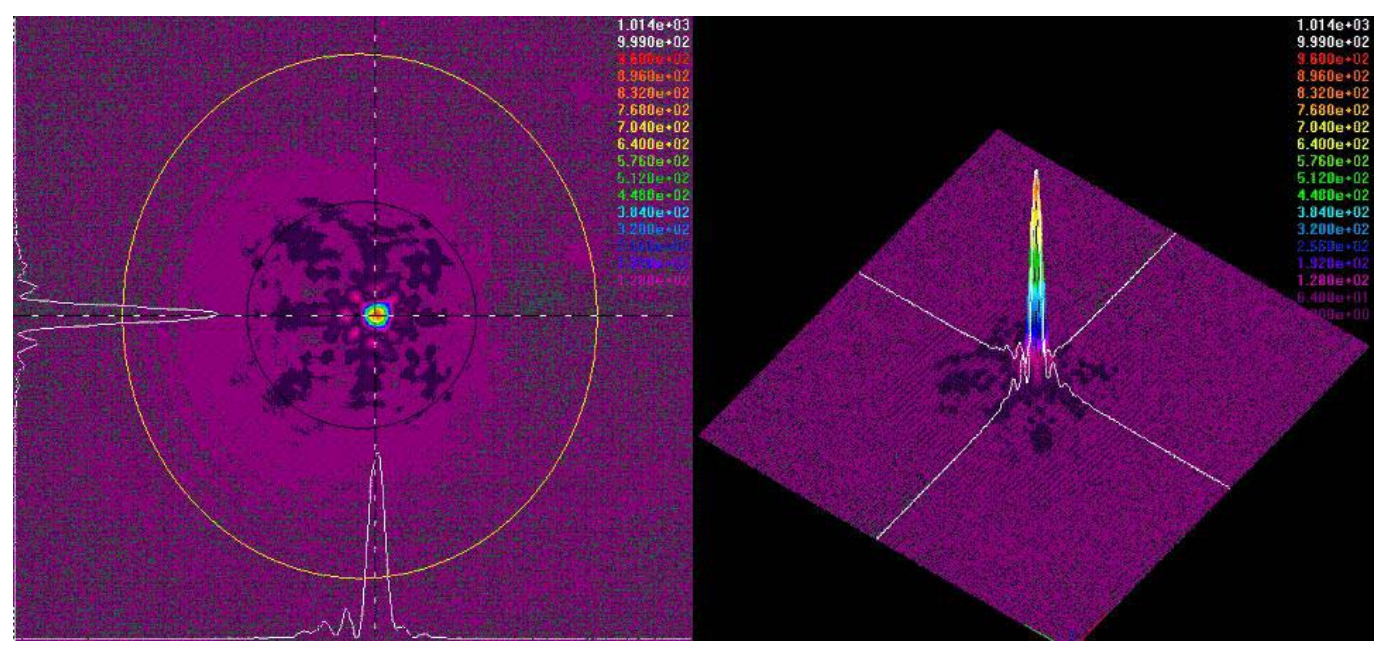

Figure 81 C4R1 Peak 2

\begin{tabular}{|c|c|c|}
\hline Lens Designation C4R1 Peak 2 & & \\
\hline Max Laser Power Through Aperture Using Objective (W) & $2.79 \mathrm{E}-04$ & \\
\hline Max Laser Power at Aperture Distance (W) & $6.87 \mathrm{E}-03$ & \\
\hline Laser Power Reduction Using Objective and Aperture (W) & $6.59 \mathrm{E}-03$ & $96 \%$ \\
\hline Magnification & 59.54 & \\
\hline Focal Disctance (mm) & 12.9794 & \\
\hline Focal range of lens (mm) & -- & \\
\hline & Avg & std \\
\hline Diameter FWHM (um) & $3.285 \mathrm{E}+00$ & $1.428 \mathrm{E}-02$ \\
\hline Diameter $13.5 \%$ (um) & $5.576 \mathrm{E}+00$ & $6.706 \mathrm{E}-02$ \\
\hline Max Lens Power Through Aperture (W) & $1.22 \mathrm{E}-03$ & \\
\hline Peak Power Density $\left(\mathrm{mW} / \mathrm{cm}^{\wedge} 2\right)$ & $1.088 \mathrm{E}+03$ & $2.464 \mathrm{E}+00$ \\
\hline Power Density FWHM $\left(\mathrm{mW} / \mathrm{cm}^{\wedge} 2\right)$ & $5.439 \mathrm{E}+02$ & $1.232 \mathrm{E}+00$ \\
\hline Power Density $13.5 \%\left(\mathrm{~mW} / \mathrm{cm}^{\wedge} 2\right)$ & $1.469 \mathrm{E}+02$ & $3.327 \mathrm{E}-01$ \\
\hline Objective Loss from 11_14_05 (W) & $7.28 \%$ & $0.86 \%$ \\
\hline Nearest Peak Power Density (W) & $1.830 \mathrm{E}+02$ & $1.465 \mathrm{E}+00$ \\
\hline SNR (based on power density) & $5.944 \mathrm{E}+00$ & $1.682 \mathrm{E}+00$ \\
\hline Efficiency & $8 \%$ & \\
\hline
\end{tabular}

Table 23 C4R1 Peak 2 


\subsubsection{Lens Column 5 Row 1}

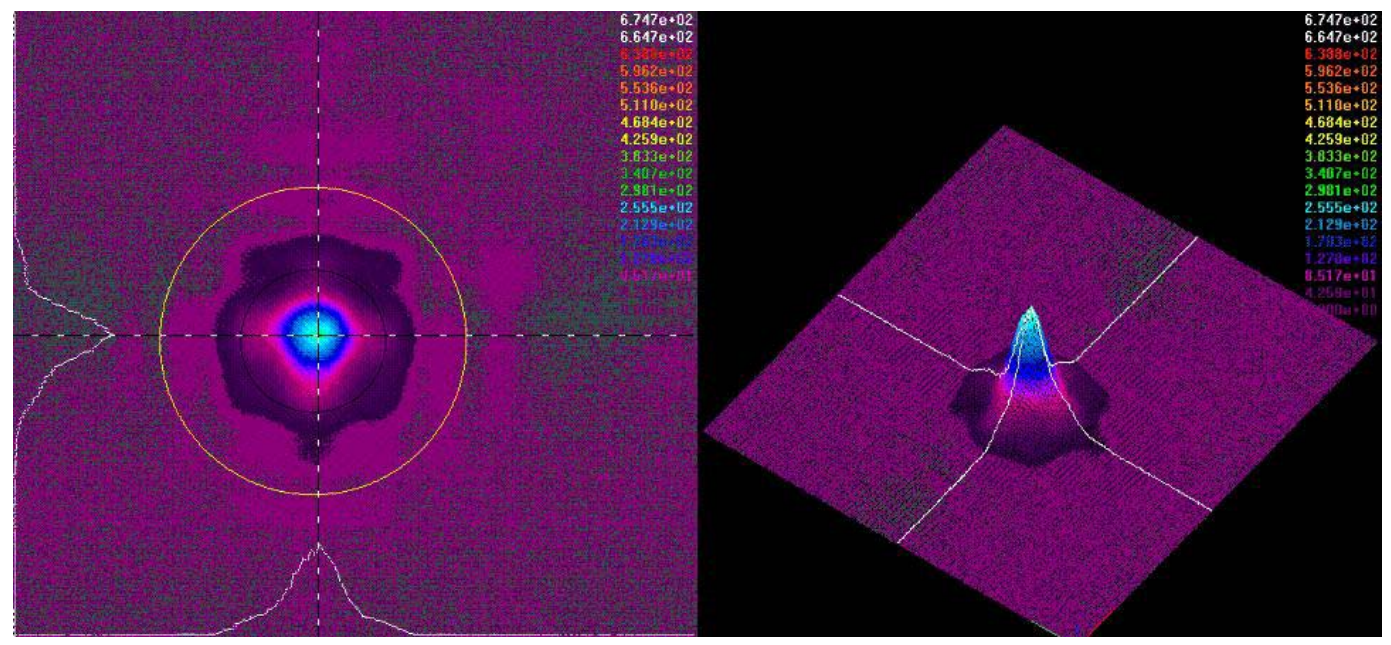

Figure 82 C5R1 Before

\begin{tabular}{|c|c|c|}
\hline \multicolumn{3}{|l|}{ Lens Designation C5R1 Before } \\
\hline Max Laser Power Through Aperture Using Objective (W) & $2.79 \mathrm{E}-04$ & \\
\hline Max Laser Power at Aperture Distance (W) & $6.87 \mathrm{E}-03$ & \\
\hline Laser Power Reduction Using Objective and Aperture (W) & $6.59 \mathrm{E}-03$ & $96 \%$ \\
\hline Magnification & 59.54 & \\
\hline Focal Disctance (mm) & 3.70205 & \\
\hline Focal range of lens (mm) & 0.73406 & \\
\hline & Avg & std \\
\hline Diameter FWHM (um) & $9.783 E+00$ & $1.018 \mathrm{E}-01$ \\
\hline Diameter $13.5 \%$ (um) & $2.366 \mathrm{E}+01$ & $1.309 \mathrm{E}-01$ \\
\hline Max Lens Power Through Aperture (W) & $1.80 \mathrm{E}-03$ & -- \\
\hline Peak Power Density $\left(\mathrm{mW} / \mathrm{cm}^{\wedge} 2\right)$ & $3.200 \mathrm{E}+02$ & $1.133 \mathrm{E}+00$ \\
\hline Power Density FWHM $\left(\mathrm{mW} / \mathrm{cm}^{\wedge} 2\right)$ & $1.600 \mathrm{E}+02$ & $5.664 \mathrm{E}-01$ \\
\hline Power Density $13.5 \%\left(\mathrm{~mW} / \mathrm{cm}^{\wedge} 2\right)$ & $4.320 E+01$ & $1.529 \mathrm{E}-01$ \\
\hline Objective Loss from 11_14_05 (W) & $7.28 \%$ & $0.86 \%$ \\
\hline Nearest Peak Power Density (W) & $\begin{array}{l}\text { unable to be } \\
\text { determined }\end{array}$ & \\
\hline SNR (based on power density) & $\begin{array}{l}\text { unable to be } \\
\text { determined }\end{array}$ & \\
\hline
\end{tabular}

Table 24 C5R1 Before 


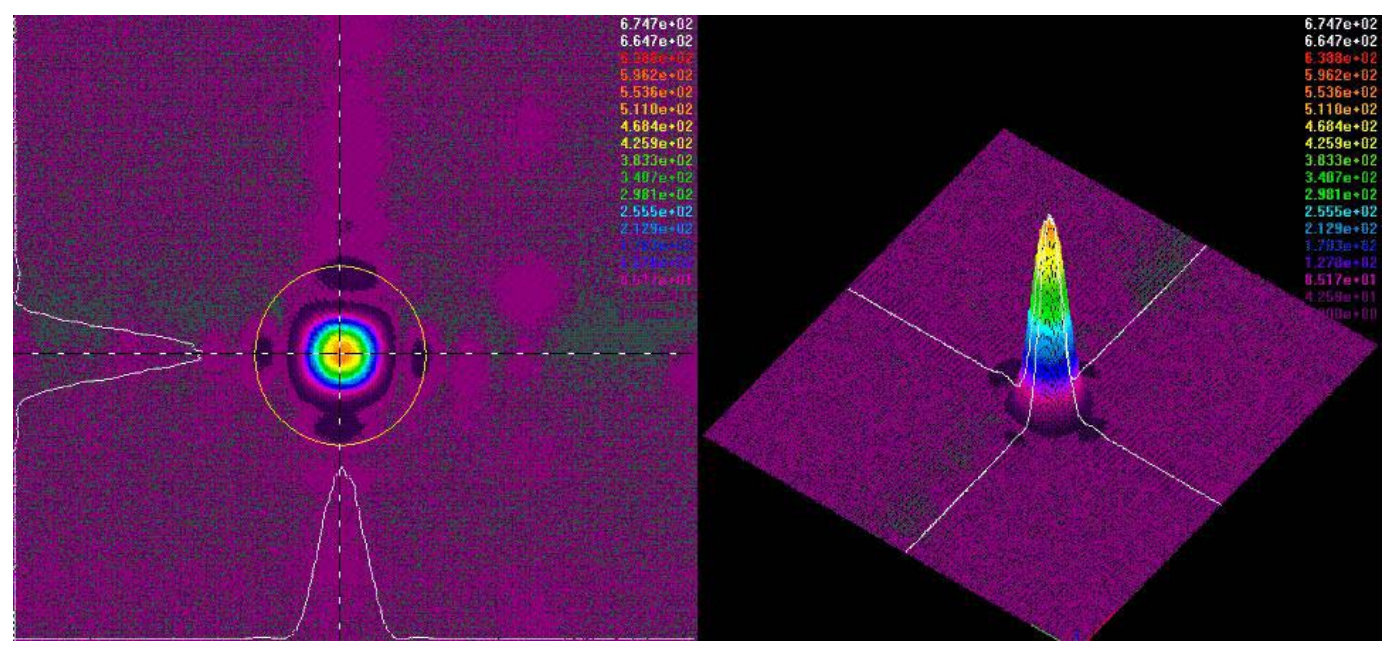

Figure 83 C5R1 Peak

\begin{tabular}{|c|c|c|}
\hline Max Laser Power Through Aperture Using Objective (W) & $2.79 \mathrm{E}-04$ & \\
\hline Max Laser Power at Aperture Distance (W) & $6.87 \mathrm{E}-03$ & \\
\hline Laser Power Reduction Using Objective and Aperture (W) & $6.59 \mathrm{E}-03$ & $96 \%$ \\
\hline Magnification & 59.54 & \\
\hline Focal Disctance (mm) & 3.9878 & \\
\hline Focal range of lens (mm) & 0.73406 & \\
\hline & Avg & std \\
\hline Diameter FWHM (um) & $8.687 \mathrm{E}+00$ & $6.545 \mathrm{E}-02$ \\
\hline Diameter $13.5 \%$ (um) & $1.387 \mathrm{E}+01$ & $6.342 \mathrm{E}-02$ \\
\hline Max Lens Power Through Aperture (W) & $1.81 \mathrm{E}-03$ & \\
\hline Peak Power Density $\left(\mathrm{mW} / \mathrm{cm}^{\wedge} 2\right)$ & $6.320 \mathrm{E}+02$ & $1.771 \mathrm{E}+00$ \\
\hline Power Density FWHM $\left(\mathrm{mW} / \mathrm{cm}^{\wedge} 2\right)$ & $3.160 \mathrm{E}+02$ & $8.856 \mathrm{E}-01$ \\
\hline Power Density $13.5 \%\left(\mathrm{~mW} / \mathrm{cm}^{\wedge} 2\right)$ & $8.532 \mathrm{E}+01$ & $2.391 \mathrm{E}-01$ \\
\hline Objective Loss from 11_14_05 (W) & $7.28 \%$ & $0.86 \%$ \\
\hline Nearest Peak Power Density (W) & $2.090 \mathrm{E}+01$ & $7.041 \mathrm{E}-01$ \\
\hline SNR (based on power density) & $3.024 \mathrm{E}+01$ & $2.516 \mathrm{E}+00$ \\
\hline Efficiency & $32 \%$ & \\
\hline
\end{tabular}

Table 25 C5R1 Peak 


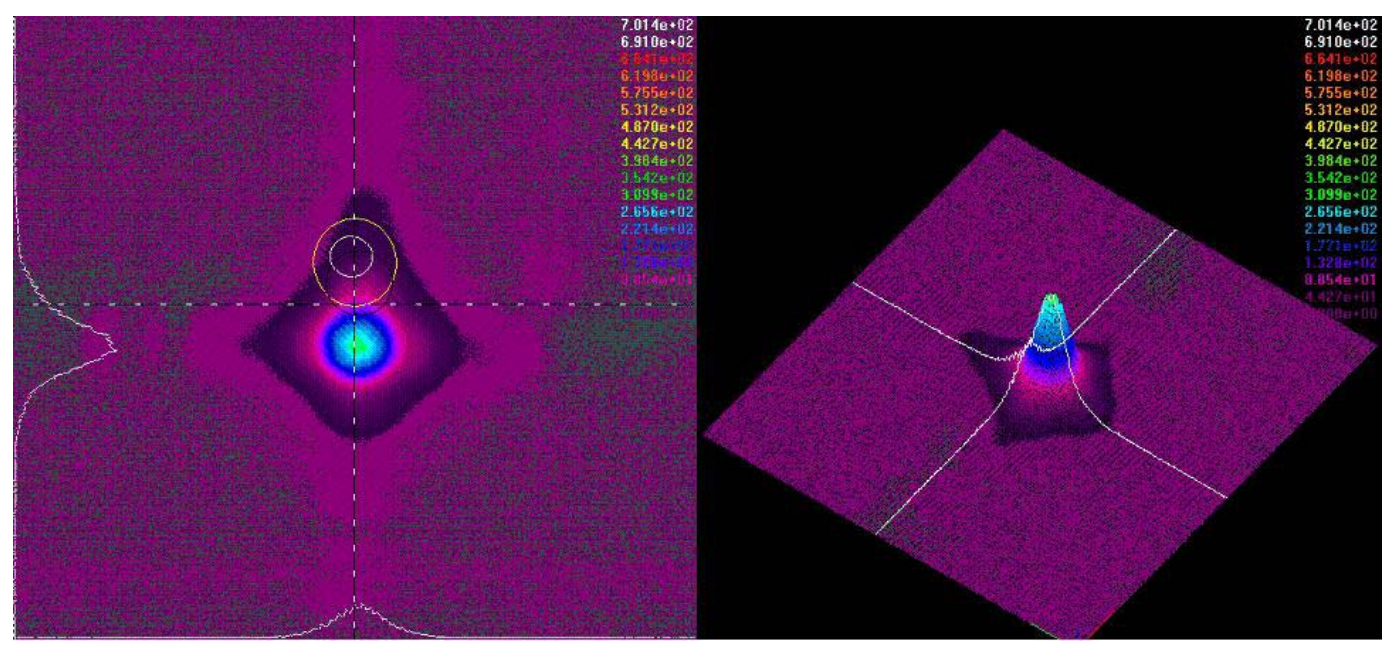

Figure 84 C5R1 After

\begin{tabular}{|c|c|c|}
\hline Max Laser Power Through Aperture Using Objective (W) & $2.79 \mathrm{E}-04$ & \\
\hline Max Laser Power at Aperture Distance (W) & $6.87 \mathrm{E}-03$ & \\
\hline Laser Power Reduction Using Objective and Aperture (W) & $6.59 \mathrm{E}-03$ & $96 \%$ \\
\hline Magnification & 59.54 & \\
\hline Focal Disctance (mm) & 4.43611 & \\
\hline Focal range of lens $(\mathrm{mm})$ & 0.73406 & \\
\hline & Avg & std \\
\hline Diameter FWHM (um) & $1.003 E+01$ & $9.605 E-02$ \\
\hline Diameter $13.5 \%$ (um) & $2.153 \mathrm{E}+01$ & $1.733 E-01$ \\
\hline Max Lens Power Through Aperture (W) & $1.80 \mathrm{E}-03$ & \\
\hline Peak Power Density $\left(\mathrm{mW} / \mathrm{cm}^{\wedge} 2\right)$ & $3.371 \mathrm{E}+02$ & $1.193 \mathrm{E}+00$ \\
\hline Power Density FWHM (mW/ $\left.\mathrm{cm}^{\wedge} 2\right)$ & $1.685 \mathrm{E}+02$ & $5.965 \mathrm{E}-01$ \\
\hline Power Density $13.5 \%\left(\mathrm{~mW} / \mathrm{cm}^{\wedge} 2\right)$ & $4.551 \mathrm{E}+01$ & $1.611 \mathrm{E}-01$ \\
\hline Objective Loss from 11_14_05 (W) & $7.28 \%$ & $0.86 \%$ \\
\hline Nearest Peak Power Density (W) & $\begin{array}{l}\text { unable to be } \\
\text { determined }\end{array}$ & \\
\hline SNR (based on power density) & $\begin{array}{l}\text { unable to be } \\
\text { determined }\end{array}$ & \\
\hline
\end{tabular}

Table 26 C5R1 After 


\subsubsection{Lens Column 6 Row 1}

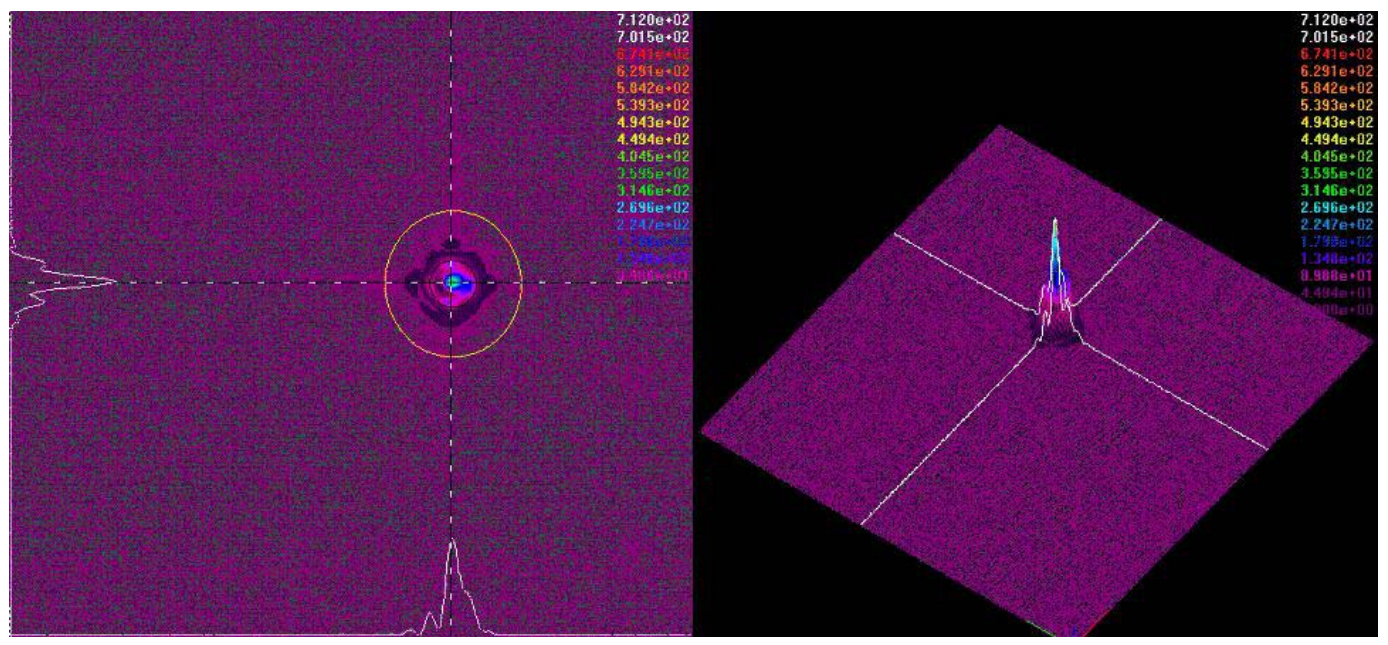

Figure 85 C6R1 Before

\begin{tabular}{|c|c|c|}
\hline \multicolumn{3}{|l|}{ Lens Designation C6R1 Before } \\
\hline Max Laser Power Through Aperture Using Objective (W) & $2.79 \mathrm{E}-04$ & \\
\hline Max Laser Power at Aperture Distance (W) & $6.87 \mathrm{E}-03$ & \\
\hline Laser Power Reduction Using Objective and Aperture (W) & $6.59 \mathrm{E}-03$ & $96 \%$ \\
\hline Magnification & 59.54 & \\
\hline Focal Disctance (mm) & 11.1506 & \\
\hline Focal range of lens $(\mathrm{mm})$ & 0.0762 & \\
\hline & Avg & std \\
\hline Diameter FWHM (um) & $2.765 E+00$ & $6.737 \mathrm{E}-02$ \\
\hline Diameter $13.5 \%$ (um) & $8.613 \mathrm{E}+00$ & $1.742 \mathrm{E}-02$ \\
\hline Max Lens Power Through Aperture (W) & $1.87 \mathrm{E}-03$ & -- \\
\hline Peak Power Density $\left(\mathrm{mW} / \mathrm{cm}^{\wedge} 2\right)$ & $3.623 E+02$ & $1.522 \mathrm{E}+00$ \\
\hline Power Density FWHM $\left(\mathrm{mW} / \mathrm{cm}^{\wedge} 2\right)$ & $1.811 \mathrm{E}+02$ & 7. $612 \mathrm{E}-01$ \\
\hline Power Density $13.5 \%\left(\mathrm{~mW} / \mathrm{cm}^{\wedge} 2\right)$ & $4.891 \mathrm{E}+01$ & $2.055 \mathrm{E}-01$ \\
\hline Objective Loss from 11_14_05 (W) & $7.28 \%$ & $0.86 \%$ \\
\hline Nearest Peak Power Density (W) & $1.641 \mathrm{E}+02$ & $1.670 \mathrm{E}+00$ \\
\hline SNR (based on power density) & $2.207 \mathrm{E}+00$ & $9.114 \mathrm{E}-01$ \\
\hline
\end{tabular}

Table 27 C6R1 Before 


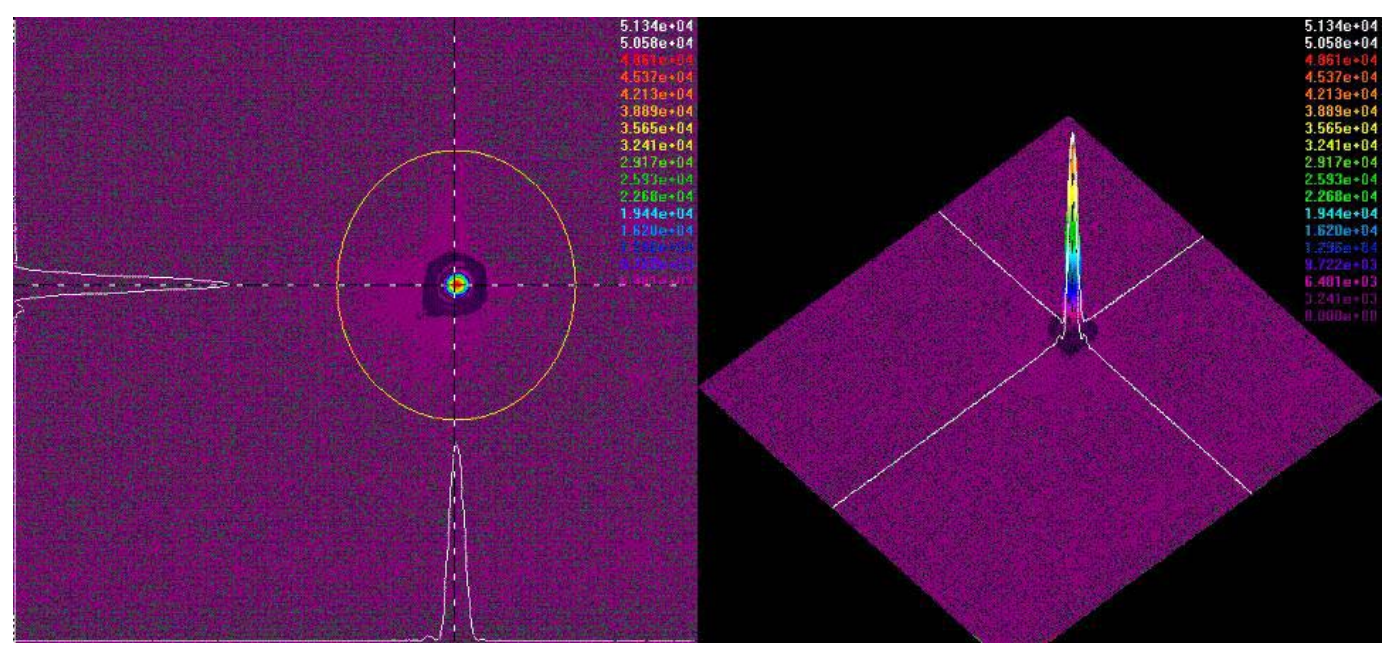

Figure 86 C6R1 Peak

\begin{tabular}{|c|c|c|}
\hline Max Laser Power Through Aperture Using Objective (W) & $2.79 \mathrm{E}-04$ & \\
\hline Max Laser Power at Aperture Distance (W) & $6.87 \mathrm{E}-03$ & \\
\hline Laser Power Reduction Using Objective and Aperture (W) & $6.59 E-03$ & $96 \%$ \\
\hline Magnification & 59.54 & \\
\hline Focal Disctance (mm) & 11.1887 & \\
\hline Focal range of lens (mm) & 0.0762 & \\
\hline & Avg & std \\
\hline Diameter FWHM (um) & $2.929 \mathrm{E}+00$ & $4.174 \mathrm{E}-02$ \\
\hline Diameter $13.5 \%$ (um) & $4.647 \mathrm{E}+00$ & $1.014 \mathrm{E}-02$ \\
\hline Max Lens Power Through Aperture (W) & $1.88 \mathrm{E}-03$ & \\
\hline Peak Power Density $\left(\mathrm{mW} / \mathrm{cm}^{\wedge} 2\right)$ & $5.309 \mathrm{E}+04$ & $1.117 \mathrm{E}+02$ \\
\hline Power Density FWHM $\left(\mathrm{mW} / \mathrm{cm}^{\wedge} 2\right)$ & $2.655 \mathrm{E}+04$ & $5.584 \mathrm{E}+01$ \\
\hline Power Density $13.5 \%\left(\mathrm{~mW} / \mathrm{cm}^{\wedge} 2\right)$ & $7.168 \mathrm{E}+03$ & $1.508 \mathrm{E}+01$ \\
\hline Objective Loss from 11_14_05 (W) & $7.28 \%$ & $0.86 \%$ \\
\hline Nearest Peak Power Density (W) & $2.574 \mathrm{E}+03$ & $4.672 \mathrm{E}+01$ \\
\hline SNR (based on power density) & $2.063 E+01$ & $2.390 \mathrm{E}+00$ \\
\hline Efficiency & $26 \%$ & \\
\hline
\end{tabular}

Table 28 C6R1 Peak 


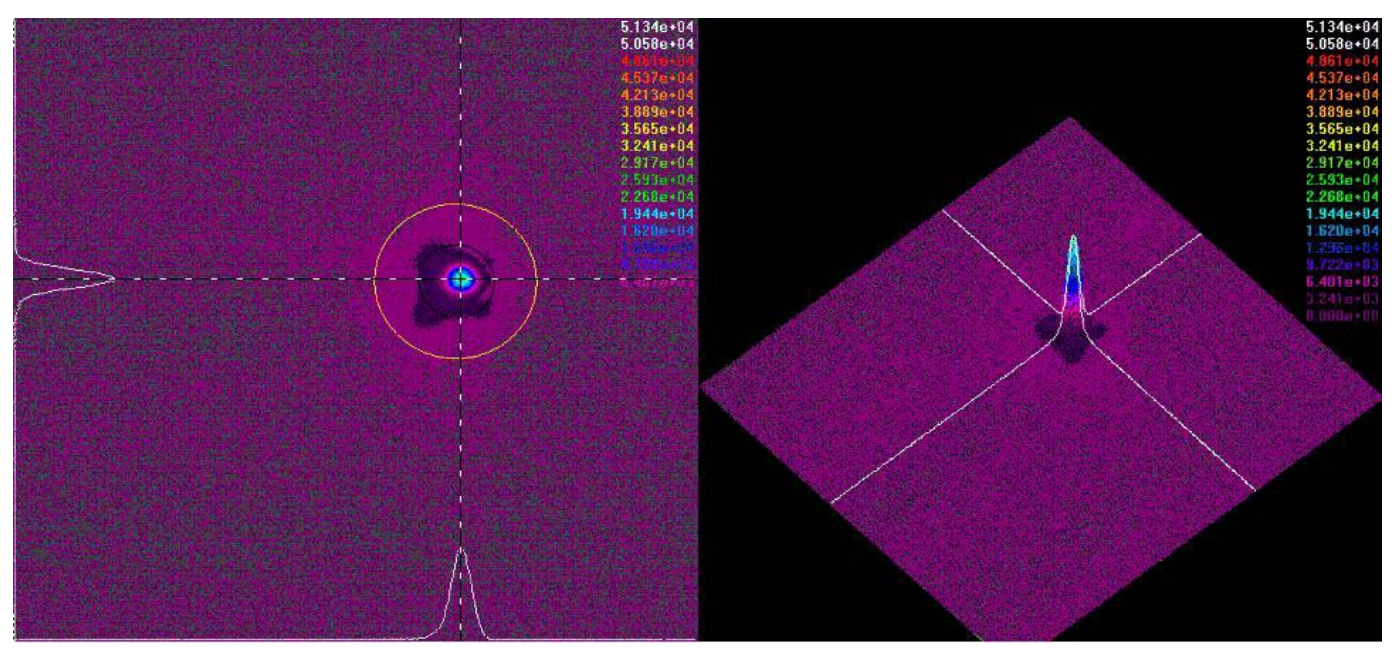

Figure 87 C6R1 After

\begin{tabular}{|c|c|c|}
\hline Max Laser Power Through Aperture Using Objective (W) & $2.79 E-04$ & \\
\hline Max Laser Power at Aperture Distance (W) & $6.87 \mathrm{E}-03$ & \\
\hline Laser Power Reduction Using Objective and Aperture (W) & $6.59 \mathrm{E}-03$ & $96 \%$ \\
\hline Magnification & 59.54 & \\
\hline Focal Disctance (mm) & 11.2268 & \\
\hline Focal range of lens (mm) & 0.0762 & \\
\hline & Avg & std \\
\hline Diameter FWHM (um) & $3.897 \mathrm{E}+00$ & $7.435 \mathrm{E}-02$ \\
\hline Diameter $13.5 \%$ (um) & $6.853 \mathrm{E}+00$ & $8.566 \mathrm{E}-02$ \\
\hline Max Lens Power Through Aperture (W) & $1.88 \mathrm{E}-03$ & \\
\hline Peak Power Density $\left(\mathrm{mW} / \mathrm{cm}^{\wedge} 2\right)$ & $2.454 \mathrm{E}+04$ & $1.006 \mathrm{E}+02$ \\
\hline Power Density FWHM $\left(\mathrm{mW} / \mathrm{cm}^{\wedge} 2\right)$ & 1.227E+04 & $5.029 \mathrm{E}+01$ \\
\hline Power Density $13.5 \%\left(\mathrm{~mW} / \mathrm{cm}^{\wedge} 2\right)$ & $3.312 \mathrm{E}+03$ & $1.358 E+01$ \\
\hline Objective Loss from 11_14_05 (W) & $7.28 \%$ & $0.86 \%$ \\
\hline Nearest Peak Power Density (W) & $\begin{array}{l}\text { unable to be } \\
\text { determined }\end{array}$ & \\
\hline SNR (based on power density) & $\begin{array}{l}\text { unable to be } \\
\text { determined }\end{array}$ & \\
\hline
\end{tabular}

Table 29 C6R1 After 


\subsubsection{Lens Column 7 Row 1}

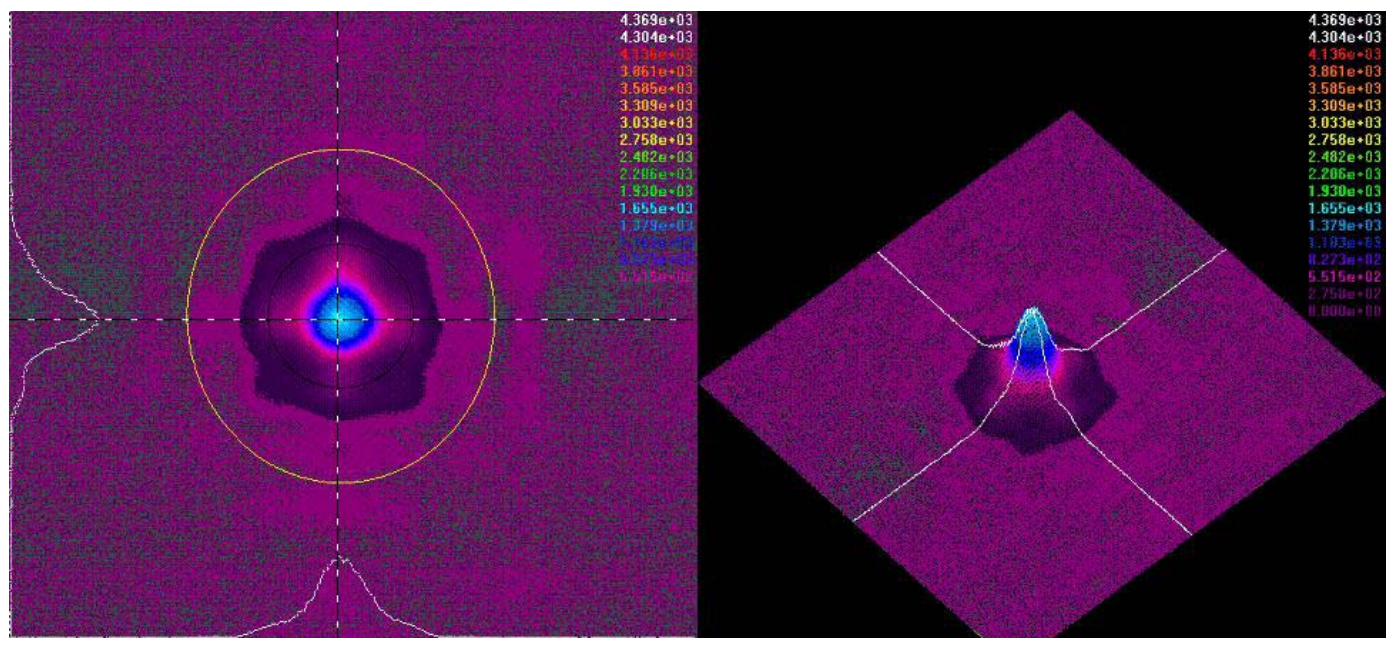

Figure 88 C7R1 Before

\begin{tabular}{|c|c|c|}
\hline Max Laser Power Through Aperture Using Objective (W) & $2.79 \mathrm{E}-04$ & \\
\hline Max Laser Power at Aperture Distance (W) & $6.87 \mathrm{E}-03$ & \\
\hline Laser Power Reduction Using Objective and Aperture (W) & $6.59 \mathrm{E}-03$ & $96 \%$ \\
\hline Magnification & 59.54 & \\
\hline Focal Disctance (mm) & 3.62585 & \\
\hline Focal range of lens $(\mathrm{mm})$ & 0.66675 & \\
\hline & Avg & std \\
\hline Diameter FWHM (um) & $1.007 \mathrm{E}+01$ & $1.124 \mathrm{E}-01$ \\
\hline Diameter $13.5 \%$ (um) & $2.526 \mathrm{E}+01$ & $1.293 E-01$ \\
\hline Max Lens Power Through Aperture (W) & $1.73 E-03$ & -- \\
\hline Peak Power Density $\left(\mathrm{mW} / \mathrm{cm}^{\wedge} 2\right)$ & $3.012 \mathrm{E}+02$ & $1.107 \mathrm{E}+00$ \\
\hline Power Density FWHM $\left(\mathrm{mW} / \mathrm{cm}^{\wedge} 2\right)$ & $1.506 \mathrm{E}+02$ & $5.536 \mathrm{E}-01$ \\
\hline Power Density $13.5 \%\left(\mathrm{~mW} / \mathrm{cm}^{\wedge} 2\right)$ & $4.067 \mathrm{E}+01$ & $1.495 \mathrm{E}-01$ \\
\hline Objective Loss from 11_14_05 (W) & $7.28 \%$ & $0.86 \%$ \\
\hline Nearest Peak Power Density (W) & $\begin{array}{l}\text { undole lo be } \\
\text { dotorminod }\end{array}$ & \\
\hline SNR (based on power density) & detormined & \\
\hline
\end{tabular}

Table 30 C7R1 Before 


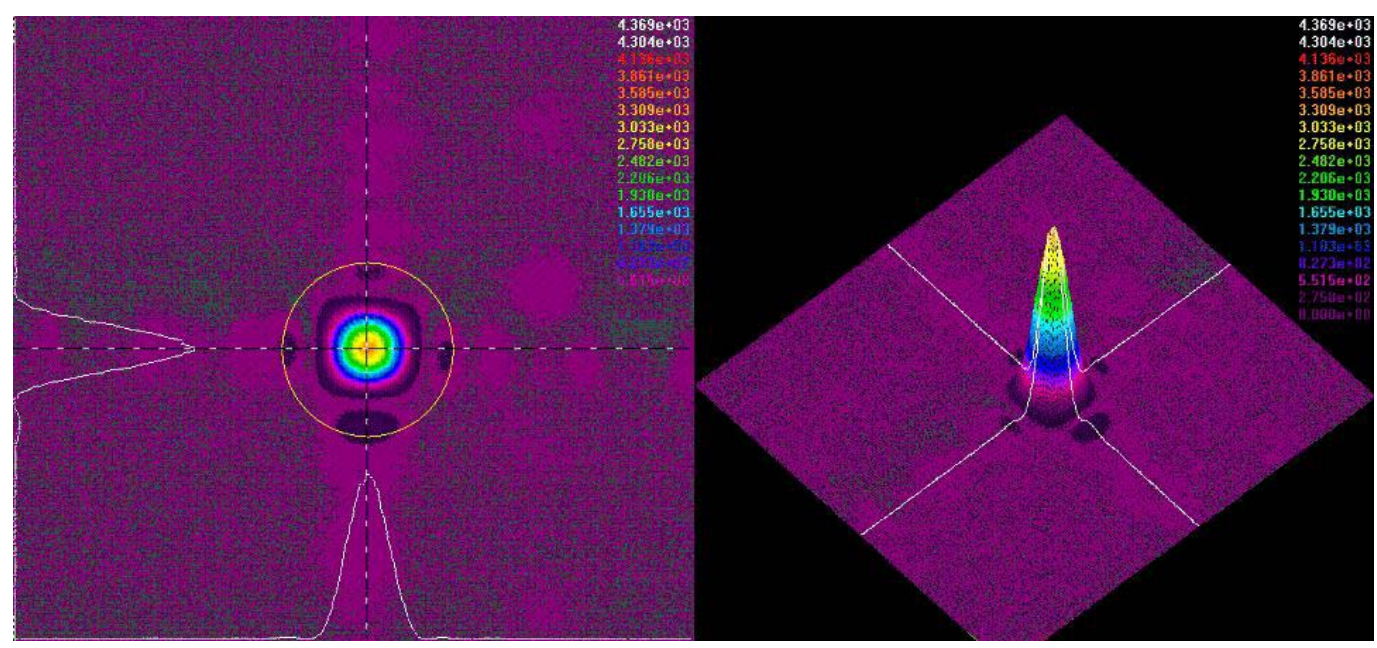

Figure 89 C7R1 Peak

\begin{tabular}{|c|c|c|}
\hline Max Laser Power Through Aperture Using Objective (W) & $2.79 \mathrm{E}-04$ & \\
\hline Max Laser Power at Aperture Distance (W) & $6.87 \mathrm{E}-03$ & \\
\hline Laser Power Reduction Using Objective and Aperture (W) & $6.59 \mathrm{E}-03$ & $96 \%$ \\
\hline Magnification & 59.54 & \\
\hline Focal Disctance (mm) & 3.9751 & \\
\hline Focal range of lens $(\mathrm{mm})$ & 0.66675 & \\
\hline & Avg & std \\
\hline Diameter FWHM (um) & $8.455 E+00$ & $5.625 \mathrm{E}-02$ \\
\hline Diameter $13.5 \%$ (um) & $1.358 \mathrm{E}+01$ & $5.067 \mathrm{E}-02$ \\
\hline Max Lens Power Through Aperture (W) & $1.76 \mathrm{E}-03$ & \\
\hline Peak Power Density $\left(\mathrm{mW} / \mathrm{cm}^{\wedge} 2\right)$ & $6.411 \mathrm{E}+02$ & $1.291 \mathrm{E}+00$ \\
\hline Power Density FWHM $\left(\mathrm{mW} / \mathrm{cm}^{\wedge} 2\right)$ & $3.206 \mathrm{E}+02$ & $6.453 E-01$ \\
\hline Power Density $13.5 \%\left(\mathrm{~mW} / \mathrm{cm}^{\wedge} 2\right)$ & $8.655 \mathrm{E}+01$ & $1.742 \mathrm{E}-01$ \\
\hline Objective Loss from 11_14_05 (W) & $7.28 \%$ & $0.86 \%$ \\
\hline Nearest Peak Power Density (W) & $2.020 \mathrm{E}+01$ & $1.934 \mathrm{E}+00$ \\
\hline SNR (based on power density) & $3.174 \mathrm{E}+01$ & $6.672 \mathrm{E}-01$ \\
\hline Efficiency & $31 \%$ & \\
\hline
\end{tabular}

Table 31 C7R1 Peak 


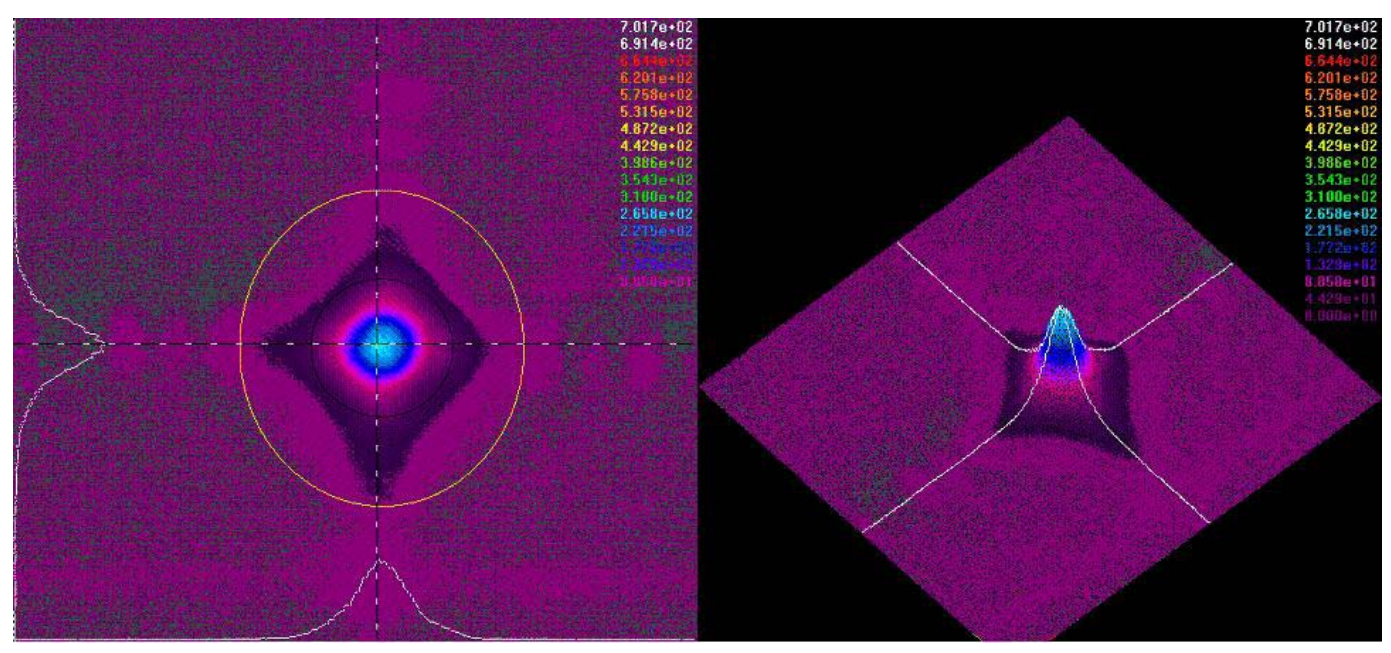

Figure 90 C7R1 After

\begin{tabular}{|c|c|c|}
\hline Max Laser Power Through Aperture Using Objective (W) & $2.79 \mathrm{E}-04$ & \\
\hline Max Laser Power at Aperture Distance (W) & $6.87 \mathrm{E}-03$ & \\
\hline Laser Power Reduction Using Objective and Aperture (W) & $6.59 \mathrm{E}-03$ & $96 \%$ \\
\hline Magnification & 59.54 & \\
\hline Focal Disctance (mm) & 4.2926 & \\
\hline Focal range of lens $(\mathrm{mm})$ & 0.66675 & \\
\hline & Avg & std \\
\hline Diameter FWHM (um) & $1.026 \mathrm{E}+01$ & $8.591 \mathrm{E}-02$ \\
\hline Diameter $13.5 \%$ (um) & $2.264 \mathrm{E}+01$ & $1.445 \mathrm{E}-01$ \\
\hline Max Lens Power Through Aperture (W) & $1.73 E-03$ & \\
\hline Peak Power Density $\left(\mathrm{mW} / \mathrm{cm}^{\wedge} 2\right)$ & $3.077 \mathrm{E}+02$ & $1.236 \mathrm{E}+00$ \\
\hline Power Density FWHM $\left(\mathrm{mW} / \mathrm{cm}^{\wedge} 2\right)$ & $1.538 \mathrm{E}+02$ & $6.179 \mathrm{E}-01$ \\
\hline Power Density $13.5 \%\left(\mathrm{~mW} / \mathrm{cm}^{\wedge} 2\right)$ & $4.154 \mathrm{E}+01$ & $1.668 \mathrm{E}-01$ \\
\hline Objective Loss from 11_14_05 (W) & $7.28 \%$ & $0.86 \%$ \\
\hline Nearest Peak Power Density (W) & $\begin{array}{l}\text { unable to be } \\
\text { determined }\end{array}$ & \\
\hline SNR (based on power density) & $\begin{array}{l}\text { unable to be } \\
\text { determined }\end{array}$ & \\
\hline
\end{tabular}

Table 32 C7R1 After 


\subsubsection{Lens Column 8 Row 1}

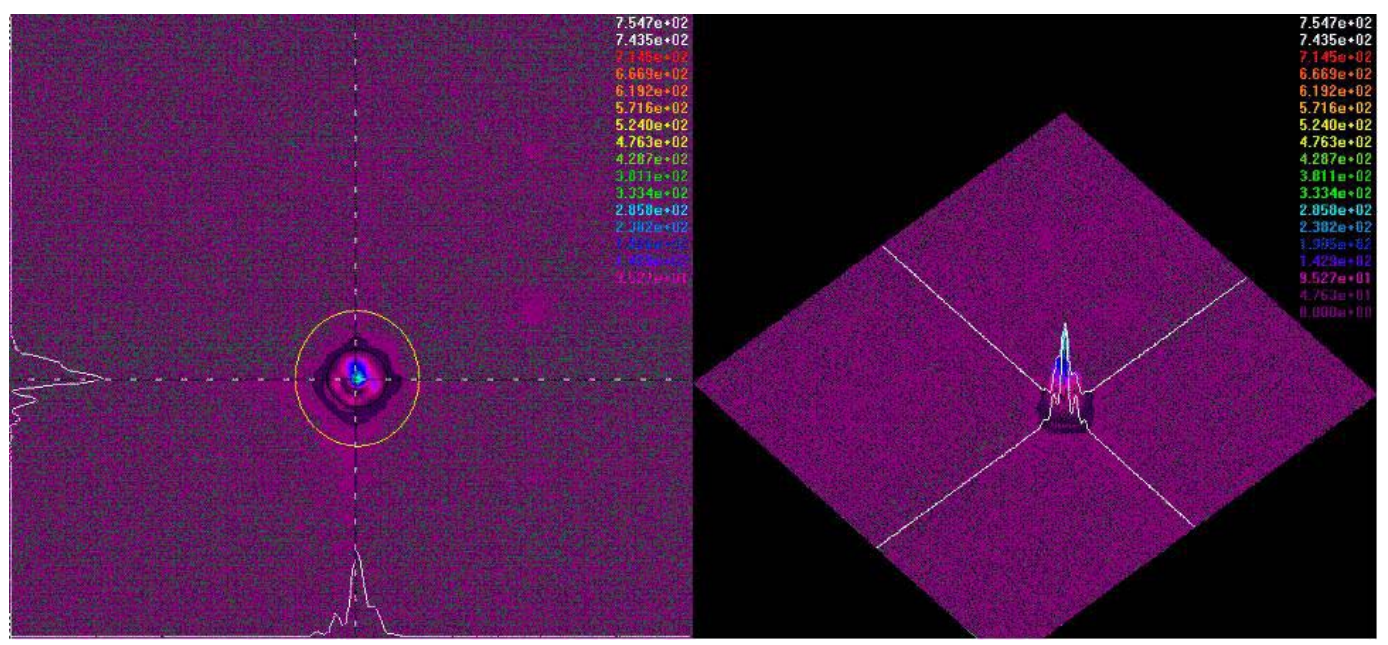

Figure 91 C8R1 Before

\begin{tabular}{|c|c|c|}
\hline Max Laser Power Through Aperture Using Objective (W) & $2.79 \mathrm{E}-04$ & \\
\hline Max Laser Power at Aperture Distance (W) & $6.87 \mathrm{E}-03$ & \\
\hline Laser Power Reduction Using Objective and Aperture (W) & $6.59 \mathrm{E}-03$ & $96 \%$ \\
\hline Magnification & 59.54 & \\
\hline Focal Disctance (mm) & 11.1379 & \\
\hline Focal range of lens (mm) & 0.0889 & \\
\hline & Avg & std \\
\hline Diameter FWHM (um) & $3.403 \mathrm{E}+00$ & $3.670 \mathrm{E}-02$ \\
\hline Diameter $13.5 \%$ (um) & $8.399 \mathrm{E}+00$ & $3.225 \mathrm{E}-02$ \\
\hline Max Lens Power Through Aperture (W) & $1.84 \mathrm{E}-03$ & -- \\
\hline Peak Power Density $\left(\mathrm{mW} / \mathrm{cm}^{\wedge} 2\right)$ & $3.379 \mathrm{E}+02$ & $1.181 \mathrm{E}+00$ \\
\hline Power Density FWHM $\left(\mathrm{mW} / \mathrm{cm}^{\wedge} 2\right)$ & $1.690 \mathrm{E}+02$ & $5.906 \mathrm{E}-01$ \\
\hline Power Density $13.5 \%\left(\mathrm{~mW} / \mathrm{cm}^{\wedge} 2\right)$ & $4.562 \mathrm{E}+01$ & $1.595 \mathrm{E}-01$ \\
\hline Objective Loss from 11_14_05 (W) & $7.28 \%$ & $0.86 \%$ \\
\hline Nearest Peak Power Density (W) & $1.745 \mathrm{E}+02$ & $1.294 \mathrm{E}+00$ \\
\hline SNR (based on power density) & $1.936 \mathrm{E}+00$ & $9.129 \mathrm{E}-01$ \\
\hline
\end{tabular}

Table 33 C8R1 Before 


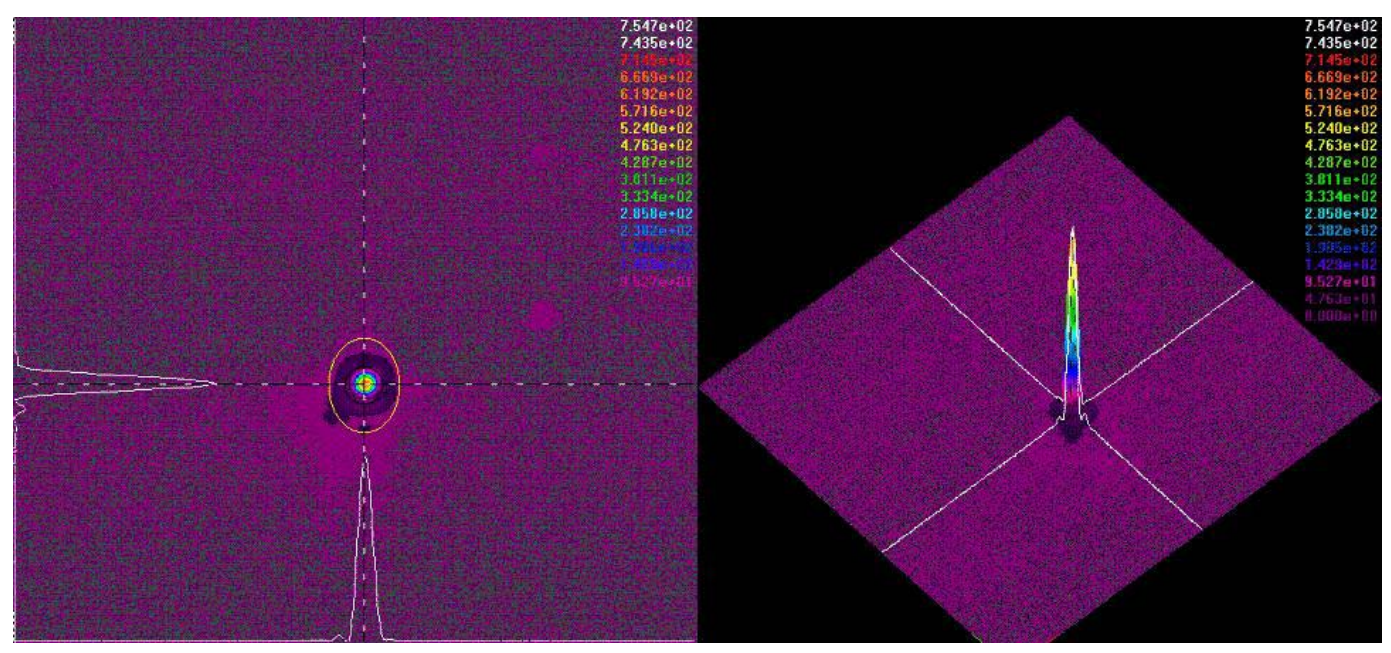

Figure 92 C8R1 Peak

\begin{tabular}{|c|c|c|}
\hline Max Laser Power Through Aperture Using Objective (W) & $2.79 \mathrm{E}-04$ & \\
\hline Max Laser Power at Aperture Distance (W) & $6.87 \mathrm{E}-03$ & \\
\hline Laser Power Reduction Using Objective and Aperture (W) & $6.59 \mathrm{E}-03$ & $96 \%$ \\
\hline Magnification & 59.54 & \\
\hline Focal Disctance (mm) & 11.176 & \\
\hline Focal range of lens (mm) & 0.0889 & \\
\hline & Avg & std \\
\hline Diameter FWHM (um) & $2.833 \mathrm{E}+00$ & $1.762 \mathrm{E}-02$ \\
\hline Diameter $13.5 \%$ (um) & $4.535 \mathrm{E}+00$ & $0.000 \mathrm{E}+00$ \\
\hline Max Lens Power Through Aperture (W) & $1.84 \mathrm{E}-03$ & \\
\hline Peak Power Density $\left(\mathrm{mW} / \mathrm{cm}^{\wedge} 2\right)$ & $5.136 \mathrm{E}+03$ & $1.166 \mathrm{E}+01$ \\
\hline Power Density FWHM (mW/cm^2) & $2.568 \mathrm{E}+03$ & $5.831 \mathrm{E}+00$ \\
\hline Power Density $13.5 \%\left(\mathrm{~mW} / \mathrm{cm}^{\wedge} 2\right)$ & $6.933 \mathrm{E}+02$ & $1.574 \mathrm{E}+00$ \\
\hline Objective Loss from 11_14_05 (W) & $7.28 \%$ & $0.86 \%$ \\
\hline Nearest Peak Power Density (W) & $3.036 \mathrm{E}+02$ & $5.797 \mathrm{E}+00$ \\
\hline SNR (based on power density) & $1.692 \mathrm{E}+01$ & $2.011 \mathrm{E}+00$ \\
\hline Efficiency & $27 \%$ & \\
\hline
\end{tabular}

Table 34 C8R1 Peak 


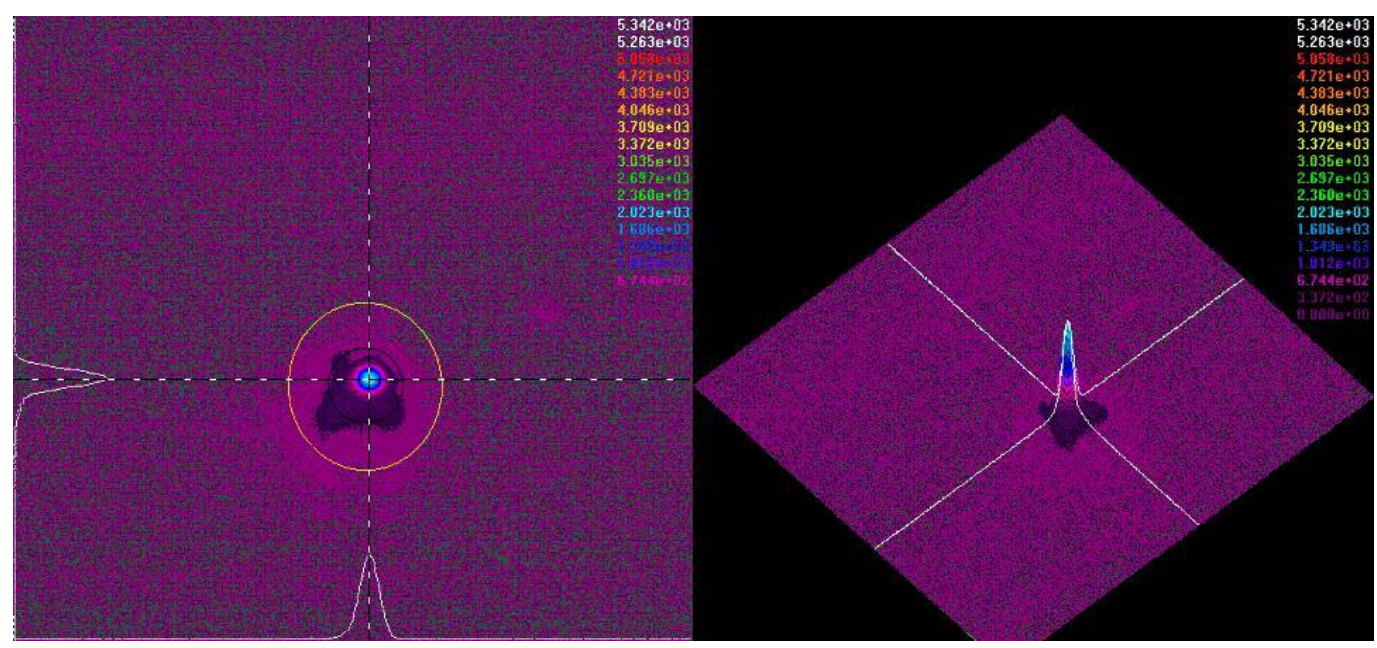

Figure 93 C8R1 After

\begin{tabular}{|c|c|c|}
\hline Max Laser Power Through Aperture Using Objective (W) & $2.79 \mathrm{E}-04$ & \\
\hline Max Laser Power at Aperture Distance (W) & $6.87 \mathrm{E}-03$ & \\
\hline Laser Power Reduction Using Objective and Aperture (W) & $6.59 \mathrm{E}-03$ & $96 \%$ \\
\hline Magnification & 59.54 & \\
\hline Focal Disctance (mm) & 11.2268 & \\
\hline Focal range of lens $(\mathrm{mm})$ & 0.0889 & \\
\hline & Avg & std \\
\hline Diameter FWHM (um) & $3.855 \mathrm{E}+00$ & $0.000 \mathrm{E}+00$ \\
\hline Diameter $13.5 \%$ (um) & $6.654 \mathrm{E}+00$ & $5.430 E-02$ \\
\hline Max Lens Power Through Aperture (W) & $1.83 \mathrm{E}-03$ & \\
\hline Peak Power Density $\left(\mathrm{mW} / \mathrm{cm}^{\wedge} 2\right)$ & $2.309 \mathrm{E}+03$ & $9.129 \mathrm{E}+00$ \\
\hline Power Density FWHM (mW/ $\left.\mathrm{cm}^{\wedge} 2\right)$ & $1.154 \mathrm{E}+03$ & $4.564 \mathrm{E}+00$ \\
\hline Power Density $13.5 \%\left(\mathrm{~mW} / \mathrm{cm}^{\wedge} 2\right)$ & $3.117 \mathrm{E}+02$ & $1.232 \mathrm{E}+00$ \\
\hline Objective Loss from 11_14_05 (W) & $7.28 \%$ & $0.86 \%$ \\
\hline Nearest Peak Power Density (W) & $\begin{array}{l}\text { unable to be } \\
\text { determined }\end{array}$ & \\
\hline SNR (based on power density) & $\begin{array}{l}\text { unable to be } \\
\text { determined }\end{array}$ & \\
\hline
\end{tabular}

Table 35 C8R1 After 


\subsubsection{Lens Comparison}

\begin{tabular}{|c|c|c|c|c|c|c|}
\hline Lens & $\begin{array}{l}\text { Focal } \\
\text { Disctance } \\
\text { (ni) }\end{array}$ & $\begin{array}{l}\text { Half Peak } \\
\text { Height } \\
\text { Focal } \\
\text { range of } \\
\text { lens (ni) }\end{array}$ & $\begin{array}{l}\text { Depth of } \\
\text { Focus (ne) }\end{array}$ & $\begin{array}{l}\text { Dianeter } \\
\text { FUHH (un) }\end{array}$ & $\begin{array}{l}\text { Dianeter } \\
13.5 \% \text { (un) }\end{array}$ & $\begin{array}{l}\text { Divergence } \\
\text { Angle } \\
\text { (rad) }\end{array}$ \\
\hline c1r1 & 4.97 & $2.223 \mathrm{E}-01$ & $4.484 \mathrm{E}-01$ & $5.299 E+00$ & 8. $450 \mathrm{E}+00$ & $4.769 \mathrm{E}-02$ \\
\hline c2r1_1 & 1.50 & -- & -- & $1.799 \mathrm{E}+00$ & $3.018 \mathrm{E}+00$ & 1. $335 \mathrm{E}-01$ \\
\hline$c 2 r 1 \_2$ & 1.91 & -- & -- & $3.211 E+00$ & $5.568 \mathrm{E}+00$ & 7. $238 \mathrm{E}-02$ \\
\hline$c 2 r 1 \_3$ & -- & -- & -- & $2.817 \mathrm{E}+00$ & $4.246 \mathrm{E}+00$ & 9. $491 \mathrm{E}-02$ \\
\hline c3r1 & 4.97 & $2.159 \mathrm{E}-01$ & 4. $998 \mathrm{E}-01$ & $5.348 \mathrm{E}+00$ & $8.446 E+00$ & 4. $771 \mathrm{E}-02$ \\
\hline $04 r 1 \_1$ & 1.50 & -- & -- & 1. $841 \mathrm{E}+00$ & $2.686 \mathrm{E}+00$ & 1. $501 \mathrm{E}-01$ \\
\hline$C 4 r 1 \_2$ & 1.90 & -- & -- & $3.285 \mathrm{E}+00$ & $5.576 \mathrm{E}+00$ & $7.227 \mathrm{E}-02$ \\
\hline $05 r 1$ & 10.70 & $7.341 \mathrm{E}-01$ & $6.415 \mathrm{E}-01$ & $8.687 \mathrm{E}+00$ & 1. $387 \mathrm{E}+01$ & 2. $905 \mathrm{E}-02$ \\
\hline $6 r 1$ & 3.82 & $7.620 \mathrm{E}-02$ & $7.199 \mathrm{E}-02$ & $2.929 \mathrm{E}+00$ & $4.647 \mathrm{E}+00$ & $8.671 \mathrm{E}-02$ \\
\hline c7r1 & 10.12 & $6.668 \mathrm{E}-01$ & $6.226 \mathrm{E}-01$ & $8.455 \mathrm{E}+00$ & 1. $358 \mathrm{E}+01$ & $2.967 \mathrm{E}-02$ \\
\hline $8 \mathrm{cr} 1$ & 3.44 & $8.890 \mathrm{E}-02$ & $7.851 \mathrm{E}-02$ & $2.833 \mathrm{E}+00$ & $4.535 \mathrm{E}+00$ & $8.886 \mathrm{E}-02$ \\
\hline
\end{tabular}

\begin{tabular}{|c|c|c|c|c|c|}
\hline Lens & $\begin{array}{l}\text { Hax Lens } \\
\text { Poper } \\
\text { Through } \\
\text { Aperture } \\
\text { (D) }\end{array}$ & $\begin{array}{l}\text { Peak Pover } \\
\text { Density } \\
(\text { (n/cn^2) }\end{array}$ & $\begin{array}{l}\text { Pover } \\
\text { Density FDHK } \\
(\text { D/ci^2) }\end{array}$ & $\begin{array}{l}\text { Pover } \\
\text { Density } \\
13.5 \% \\
(\text { ㅍ/cn`2) }\end{array}$ & $\begin{array}{l}\text { SHR (based } \\
\text { on pover } \\
\text { density) }\end{array}$ \\
\hline c1r1 & $1.362 \mathrm{E}-03$ & $1.003 \mathrm{E}+04$ & $5.014 \mathrm{E}+03$ & $1.354 \mathrm{E}+03$ & 9.57 \\
\hline c2r1_1 & 1.117E-03 & $9.680 \mathrm{E}+02$ & $4.840 \mathrm{E}+02$ & $1.307 \mathrm{E}+02$ & 4.40 \\
\hline c $2 r 1 \_2$ & 1. $126 \mathrm{E}-03$ & $1.049 \mathrm{E}+03$ & $5.247 \mathrm{E}+02$ & 1. $417 \mathrm{E}+02$ & 6.02 \\
\hline c2r1_3 & $1.102 \mathrm{E}-03$ & $1.159 \mathrm{E}+03$ & $5.793 \mathrm{E}+02$ & $1.564 \mathrm{E}+02$ & 5.62 \\
\hline c3r1 & 1. $541 \mathrm{E}-03$ & 1. $212 \mathrm{E}+03$ & $6.061 \mathrm{E}+02$ & $1.637 \mathrm{E}+02$ & 8.68 \\
\hline $04 r 1 \_1$ & $1.277 \mathrm{E}-03$ & $1.091 \mathrm{E}+03$ & $5.455 \mathrm{E}+02$ & 1. $473 \mathrm{E}+02$ & 4.22 \\
\hline C4r1_2 & 1. $224 \mathrm{E}-03$ & $1.088 \mathrm{E}+03$ & $5.439 E+02$ & $1.469 \mathrm{E}+02$ & 5.94 \\
\hline $05 r 1$ & 1. $812 \mathrm{E}-03$ & $6.320 \mathrm{E}+02$ & $3.160 \mathrm{E}+02$ & $8.532 \mathrm{E}+01$ & 30.24 \\
\hline $6 r 1$ & 1.879E-03 & $5.309 E+04$ & $2.655 \mathrm{E}+04$ & $7.168 \mathrm{E}+03$ & 20.63 \\
\hline$c 7 r 1$ & 1. $758 \mathrm{E}-03$ & $6.411 \mathrm{E}+02$ & $3.206 \mathrm{E}+02$ & $8.655 E+01$ & 31.74 \\
\hline c8r1 & 1. $840 \mathrm{E}-03$ & $5.136 \mathrm{E}+03$ & $2.568 \mathrm{E}+03$ & $6.933 E+02$ & 16.92 \\
\hline
\end{tabular}

\begin{tabular}{|c|c|c|c|c|c|}
\hline Lens & $\begin{array}{l}\text { Lens } \\
\text { (strict) } \\
\text { Ef ficiency }\end{array}$ & $\begin{array}{l}\text { Ef f iciency } \\
\text { of } \\
\text { Borof loat } \\
(\mathrm{Avg})\end{array}$ & $\begin{array}{l}\text { Lens } \\
\text { Ef f iciency } \\
\text { (corrected) }\end{array}$ & \begin{tabular}{|l|} 
Average \\
Pover \\
of lens \\
Focal \\
Point (प) \\
\end{tabular} & $\begin{array}{l}\text { Percent } \\
\text { Peak Pover } \\
\text { Density } \\
\text { Increase } \\
\end{array}$ \\
\hline c1r1 & $18 \%$ & $92 \%$ & $20 \%$ & $1.035 \mathrm{E}-03$ & $1145 \%$ \\
\hline c2r1_1 & $2 \%$ & $92 \%$ & $2 \%$ & 1. $228 \mathrm{E}-04$ & $20 \%$ \\
\hline$c 2 r 1 \_2$ & $7 \%$ & $92 \%$ & $7 \%$ & $3.898 \mathrm{E}-04$ & $30 \%$ \\
\hline$c 2 r 1 \_3$ & $6 \%$ & $92 \%$ & $6 \%$ & $3.151 \mathrm{E}-04$ & $44 \%$ \\
\hline $\mathrm{c} 3 \mathrm{r} 1$ & $20 \%$ & $92 \%$ & $22 \%$ & $1.155 \mathrm{E}-03$ & $51 \%$ \\
\hline $04 \mathrm{r} 1 \_1$ & $2 \%$ & $92 \%$ & $3 \%$ & 1. $328 \mathrm{E}-04$ & $36 \%$ \\
\hline $04 r 1 \_2$ & $7 \%$ & $92 \%$ & $8 \%$ & 4.039E-04 & $35 \%$ \\
\hline $05 r 1$ & $29 \%$ & $92 \%$ & $31 \%$ & $1.625 \mathrm{E}-03$ & $22 \%$ \\
\hline c6r1 & $23 \%$ & $92 \%$ & $26 \%$ & $1.334 \mathrm{E}-03$ & $6494 \%$ \\
\hline c7r1 & $28 \%$ & $92 \%$ & $30 \%$ & $1.565 \mathrm{E}-03$ & $20 \%$ \\
\hline c8r1 & $24 \%$ & $92 \%$ & $26 \%$ & $1.380 \mathrm{E}-03$ & $538 \%$ \\
\hline
\end{tabular}

Table 36 Summary of Row 1 on-axis lens characterization 


\subsection{Discussion of Lens Results and Connection to Diffraction Theory}

The focal length, spot size, SNR, and efficiency results for the on-axis lenses have been shown above to correspond to their intended design parameters. Knowledge of these quantities are needed to implement an integrated optical monitoring design. The efficiency of $20-30 \%$ falls short of the $40.5 \%$ efficiency upper limit for a binary profile [22] and could possibly be due to damage, fabrication conditions, or the small feature sizes of the lenses. Larger feature sizes may account for the slightly higher efficiency of lenses C5R1 and C7R1 compared to C6R1 and C8R1 which have smaller features. Making the lenses in a clean room may improve the lens efficiency even further. The lens focal points match closely to their intended design. The observed focal points of lenses 5-7 are approximately twice their intended value. This is possibly due to the use of $633 \mathrm{~nm}$ light used to characterize these lenses when they were designed for $1310 \mathrm{~nm}$ light.

A more useful lens for integrated optical monitoring is one that can produce dual, off-axis spots. These types of lenses were also developed [1] along with the on-axis lenses. Those types of lenses reduce the number of lenses needed to test multiple MEMS devices and allow for the use of multiple lenses to probe a single device. The dual spot lenses would also allow

for a reference beam and measurement beam setup for a single device as has been used on the laser Doppler vibrometer in previous work [14]. The tests and analysis used on the on-axis lenses can be applied to the dual spot, off-axis lenses for testing their ability to detect motion of MEMS devices. The off-axis lenses have the added difficulty of being able to return the reflected light back to the input fiber for analysis and providing enough laser power to produce a reflected beam that can be detected by the vibrometer. This is because the off axis spot would produce a reflected beam that would not travel along the same path as the incident beam.

The lenses, being diffractive in nature, produce side lobes that can vary in intensity depending on the state of the lens (damaged or undamaged), the edges of each lens, and the nature of diffracted light. Each lens pattern begins with large rings at its center. The rings in the lens 
pattern are smaller for distances farther from the lens center as seen in Figure 69. The rings on the edges of some lenses become small enough to make their fabrication difficult. This can lead to improperly formed outer rings near the edge of those lenses. Any improperly formed edge or damaged ring of a lens detracts from the intended diffraction pattern. Each diffraction ring forms wave fronts that add together to produce the diffraction pattern in the far field. Removal of one of these added light waves causes the diffraction pattern to change. Side lobes of light can also be observed in diffraction patterns produced by a circular aperture or other simple aperture. The diffraction pattern of any aperture in the far field is the spatial Fourier transform of the aperture opening. For the case of a single circular aperture [10,21], the output looks similar to a $\operatorname{sinc}(\mathrm{x})$ function that is circularly symmetric about the transverse plane of the beam.

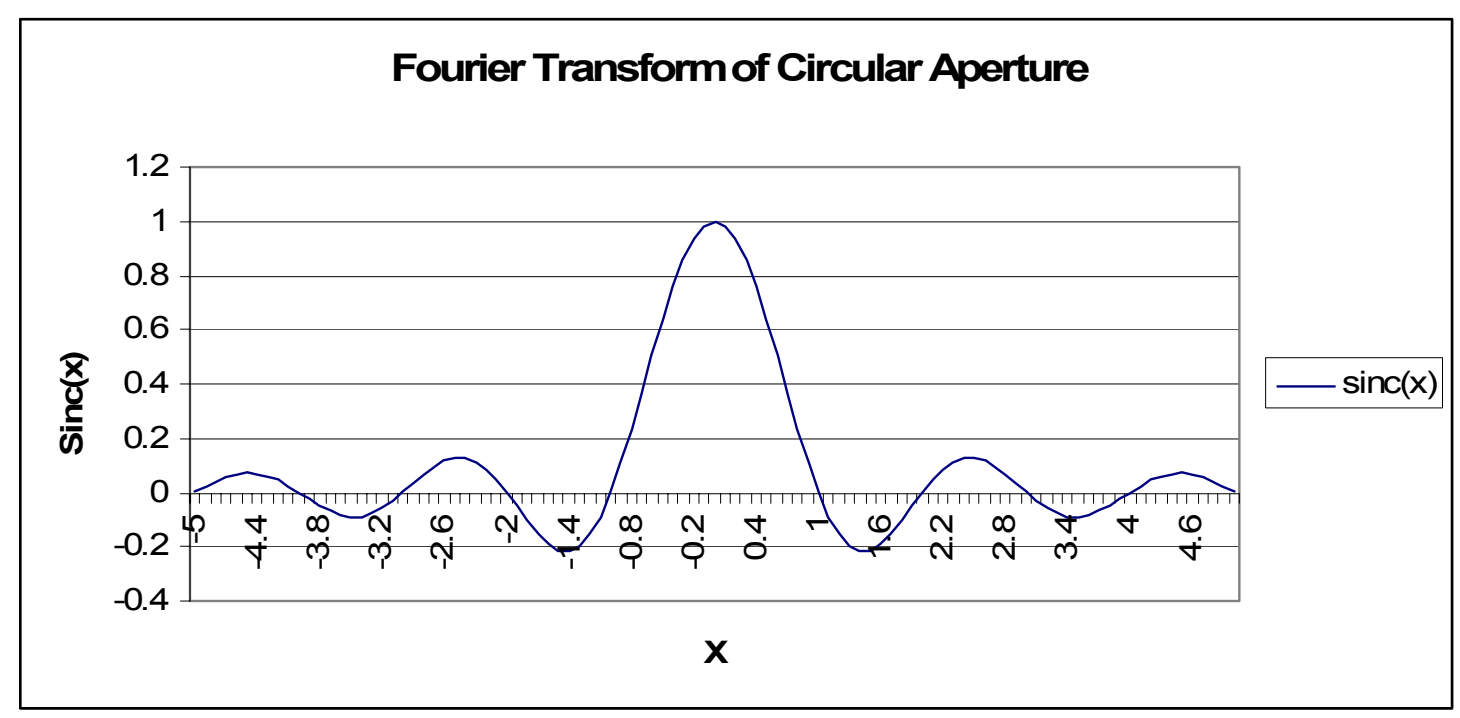

Figure 94 Example diffraction pattern formed from a circular aperture

The addition of multiple rings of the correct dimensions around the a central circular aperture can add to diminish, but not entirely remove the side lobes through light interference. These added rings also narrow the width of them main peak of the single circular aperture. The reason for this can be thought of qualitatively by considering a one dimensional, rectangular slit aperture. We can consider the rectangular aperture as the sum of shifted, uniformly weighted delta functions of the form:

$$
\delta\left(\mathrm{t}-\mathrm{t}_{0}\right)
$$


whose Fourier transforms are of the form:

$$
\exp \left(-\mathrm{j} 2 \pi \mathrm{ft}_{0}\right)=\cos \left(2 \pi \mathrm{ft}_{0}\right)-\mathrm{j} * \sin \left(2 \pi \mathrm{ft}_{0}\right)
$$

Adding the Fourier transform of these shifted delta functions over the aperture produces the intensity pattern in the far field that is the Fourier transform of the aperture. You could also view this as an extension of the Huygens- Fresnel principle mentioned in Chapter 6. Adding a second rectangular aperture that is close to the first according to the grating equation [8]

$$
\begin{aligned}
& d \sin \theta=m \lambda \\
& m=0, \pm 1, \pm 2, \ldots
\end{aligned}
$$

where $\mathrm{m}$ is the diffraction order, will provide constructive interference of light from both apertures. We can now think of adding multiple rectangular, one dimensional apertures spaced according to the grating equation and rotating them about a central circular aperture. Each ring would produce the sum of Fourier transforms of an infinite number of zero width one dimensional, rectangular apertures over $2 \pi$ radians or equivalently, the integral of the rectangular aperture over $2 \pi$ radians. This integration gives the Fourier transform of one ring. The DOE diffraction pattern can then be constructed from the sum of the Fourier transform from all of the rings added to the Fourier transform of the central circular aperture. This is the conceptual principle from which the diffractive lenses discussed in this thesis operate. 


\section{Chapter 9}

\section{Lens Simulation Efforts}

An attempt was made to use the previously created Mat-lab program to simulate the behavior of the lens output on either side of the lens focal plane. The original intent of the program was to design a Fresnel lens profile that would produce a focused spot in a specified focal plane. The previous sections of this thesis indicate that it did this successfully. Using the program to predict the output of the lens on either side of the focal plane proved to be inconclusive. This is because the simulation was compared to lens C2R1. This was not within the scope of the project at the time and was set aside to move on with characterization. Ideally, a suitably adapted program should be able to predict output results around the focal plane where the input file could potentially incorporate fabrication defects to give a more accurate prediction of the diffracted output after fabrication. 


\section{Chapter 10}

\section{Vibrometer Tests}

\subsection{Laser Doppler Vibrometry Theory}

The following discussion is adapted from the Vibrometer operating manual[20]. The laser Doppler vibrometer detects motion by detecting the phase difference of two coherent light beams that coincide. This phase difference between the two beams causes a variation in the received intensity at the photo detector. The intensity is given as a function of the phase difference by:

$$
\begin{aligned}
& I(\Delta \varphi)=\frac{I_{\max }}{2}(1+\cos (\Delta \varphi)) \\
& \Delta \varphi=2 \pi \frac{\Delta L}{\lambda}
\end{aligned}
$$

Where $\Delta L$ is the optical path length difference between the two beams and $\lambda$ is the wavelength of the laser. This path difference can be a function of time if the the light from one of the two beams is scattered back. This scattered light creates an interference pattern. Movement of this pattern can be detected by using directionally sensitive counting of the fringe pattern. The scattered light is subject to a Doppler frequency shift given by:

$$
f_{d}=\frac{2|v|}{\lambda}
$$


The reflected light can then be superimposed onto light at a known frequency (reference beam) which causes a beating pattern to be received at the detector. This beating pattern is caused by the frequency difference between the two waves. The above equation is independent of sign. Light of a fixed frequency shift $\mathrm{f}_{\mathrm{b}}$ is added to it so the velocity direction can be determined.

$$
f_{\bmod }=f_{b}+\frac{2 v}{\lambda}
$$

These directionally sensitive interferometers are called heterodyne interferometers.

OFV-512 The optical configuration of the sensor head OFV-512 is shown schematically in figure C.3.

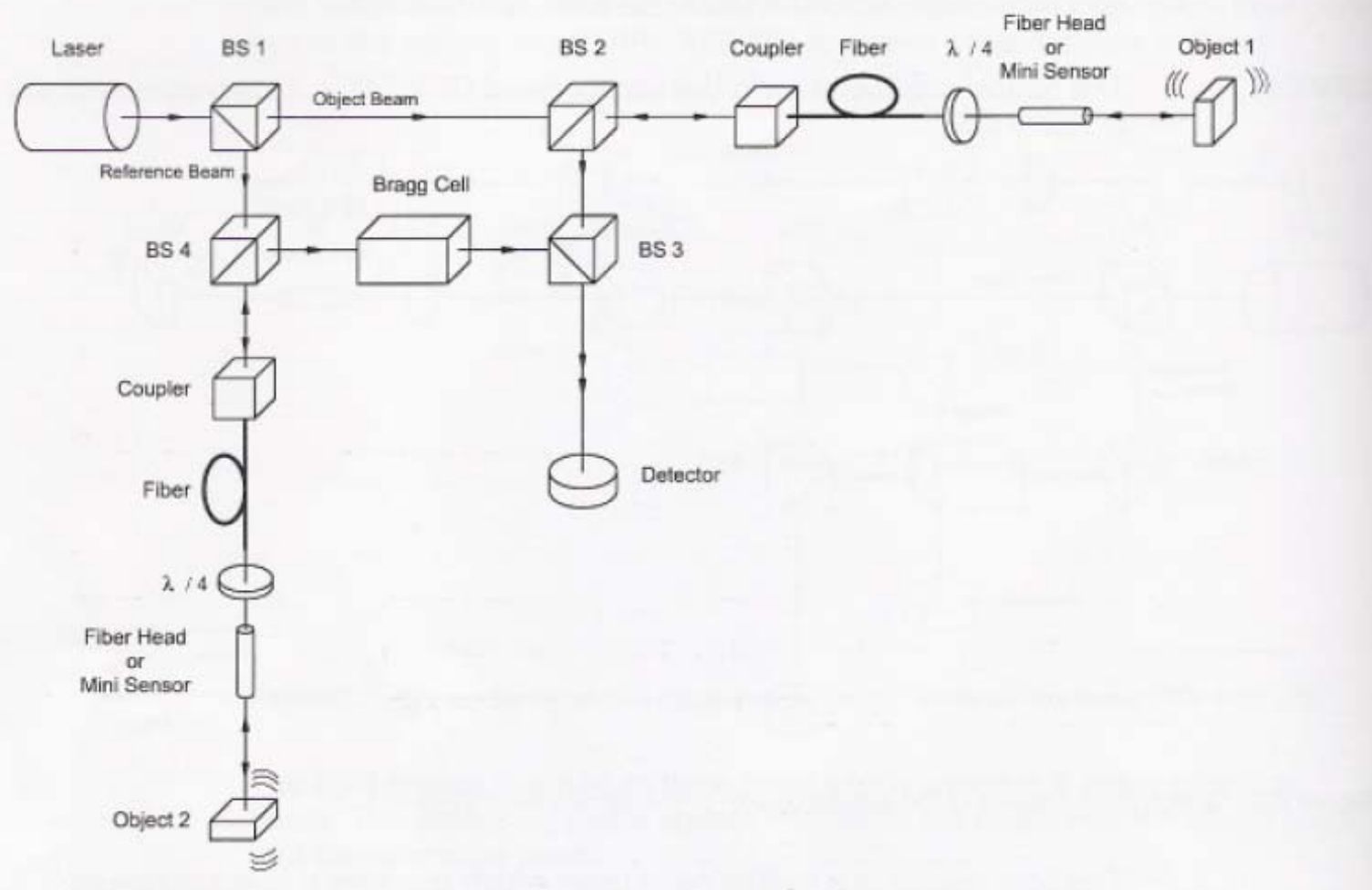

Figure C.3: Optical configuration of the interferometer in the sensor head OFV-512

Figure 95 Layout of laser Doppler vibrometer [20] 


\subsection{Test Setup}

The vibrometer was used to test the viability of using the lens to transfer a laser spot to and from an object for use in motion tracking. The following is a picture of the vibrometer and lens setup.
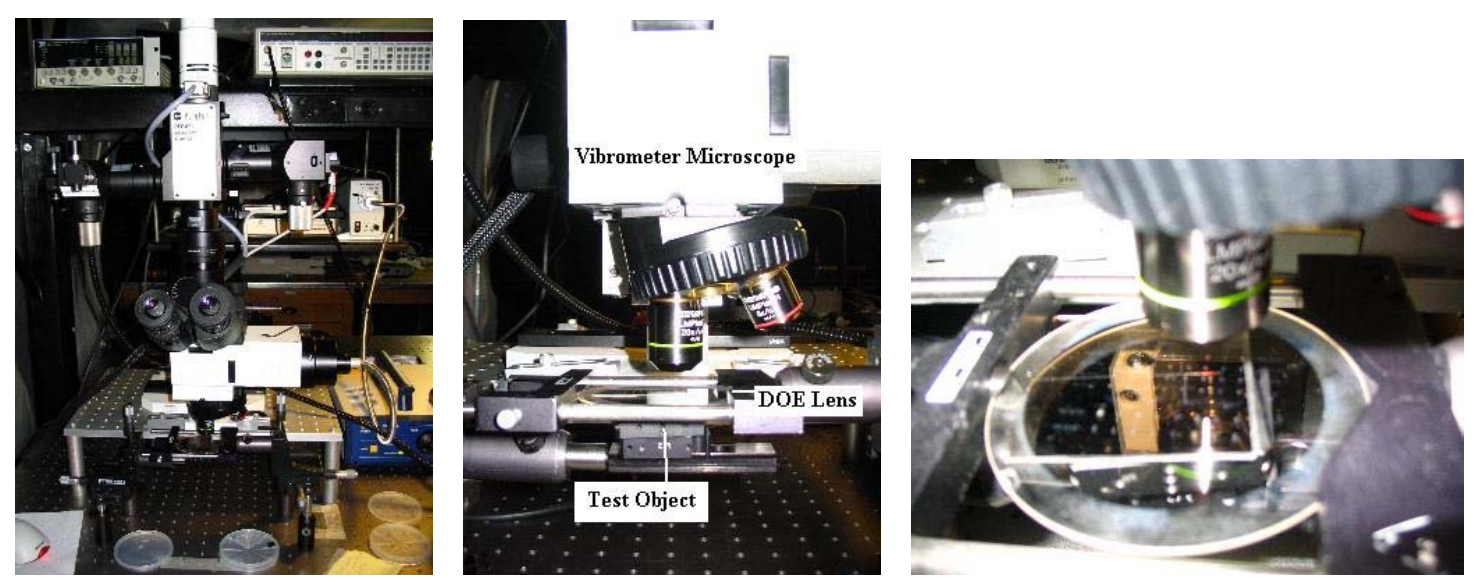

Figure 96 Left to Right: Vibrometer, DOE, and test object setup

The Polytec vibrometer uses a $633 \mathrm{~nm}, 1 \mathrm{~mW}$ laser to take measurements. It was set up to be used as a two fiber system where one fiber was a reference beam and the other the test beam. This caused no feed back signal to be received from the DOE lenses since the light from both fibers could not be coupled into a single DOE lens to give enough reflected power to take measurements. The vibrometer user manuals indicated that a terminating part (OFV-151) could be placed onto one of the fibers to convert it into a single fiber measuring system. This allowed enough signal power to be received back into the vibrometer to test the DOE as a focusing lens.

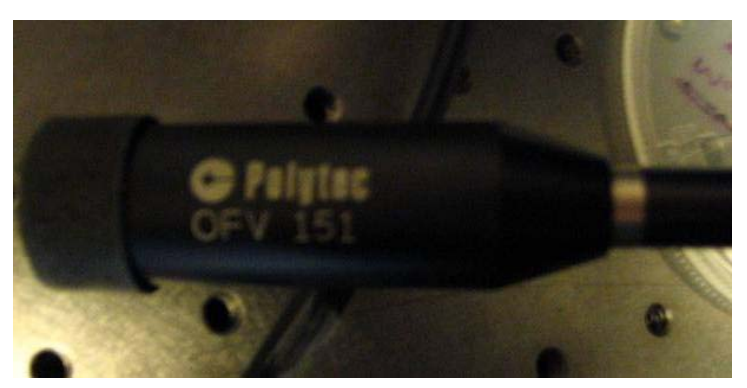

Figure 97 Fiber terminating piece to allow one fiber operation of vibrometer. 
The focal plane of the DOE lens was placed at the focal point of the vibrometer microscope objective to see if the lens could focus a spot and transfer it to the vibrometer.

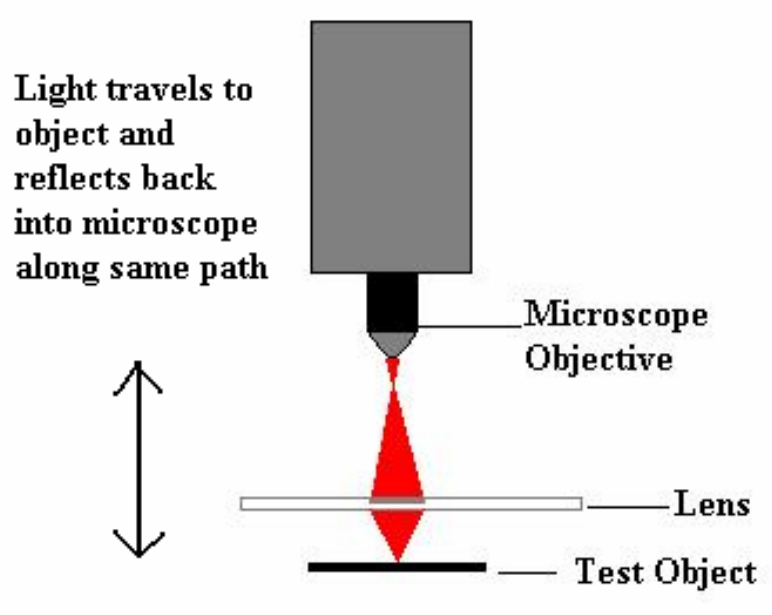

Figure 98 Lens-Vibrometer test setup

This test configuration allows visual images of the test object to be displayed on a computer screen.

\subsection{Lens Imaging Tests}

There are multiple reflections for the DOE lenses which cause multiple images to be seen. The following images were gathered from lens C6R1. This lens gave the best results and has one of the highest efficiency ratings as gathered from earlier tests. The test object is an old MEM lateral comb resonator contact pad. The first image is produced when the focal plane of the objective is placed on the test object. The objective's focal plane is then moved upward from the sample and toward the main focal plane which resides above the DOE wafer. 


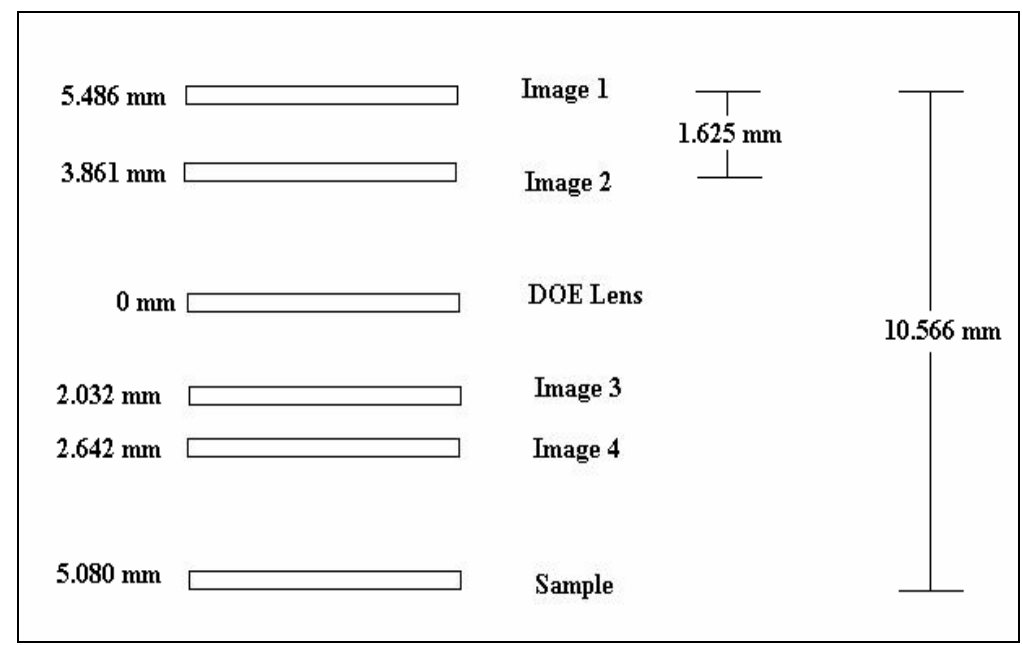

Figure 99 Layout of images seen with DOE in vibrometer setup

Each lens has multiple reflections and images resulting from the glass and DOE structure. The Figure 99 shows observed images using lens C6R1 and their associated distances from the DOE wafer. The image at the top is the expected focal point of the lens and produces a reversed image of the sample observed. This reversal is indicative of a single lens which the DOE simulates. The other images are thought to be reflections since the images observed are not reversed. Image 3 is the clearest while Image 1 is the largest. The pictures below are the images of the sample as seen through the DOE lens. The images were transferred to the vibrometer for visual inspection. The sample image is of a lateral comb resonator.

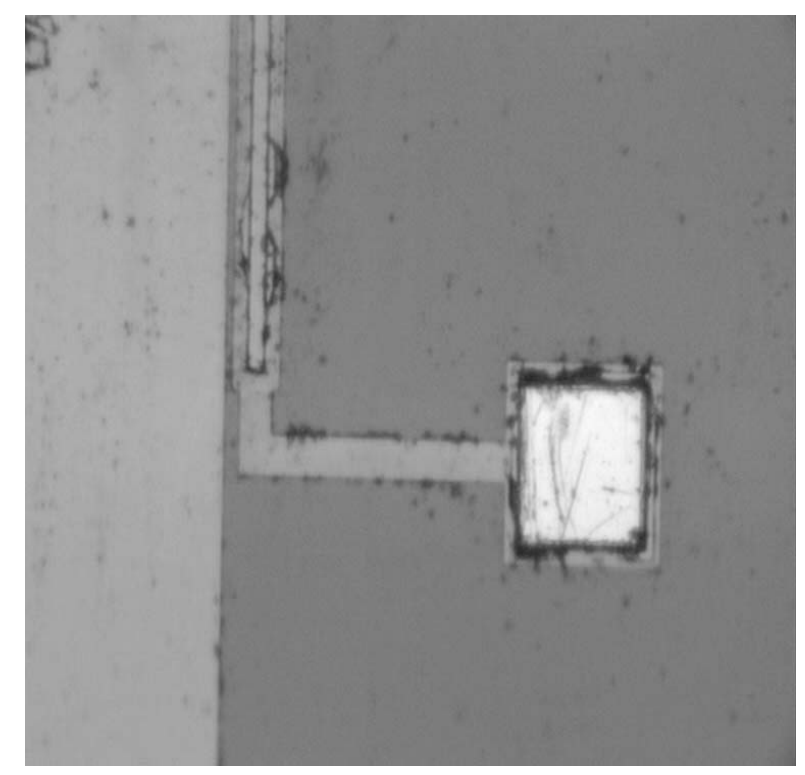

Figure 100 Sample lateral comb resonator contact pad viewed at 20x with the vibrometer microscope 


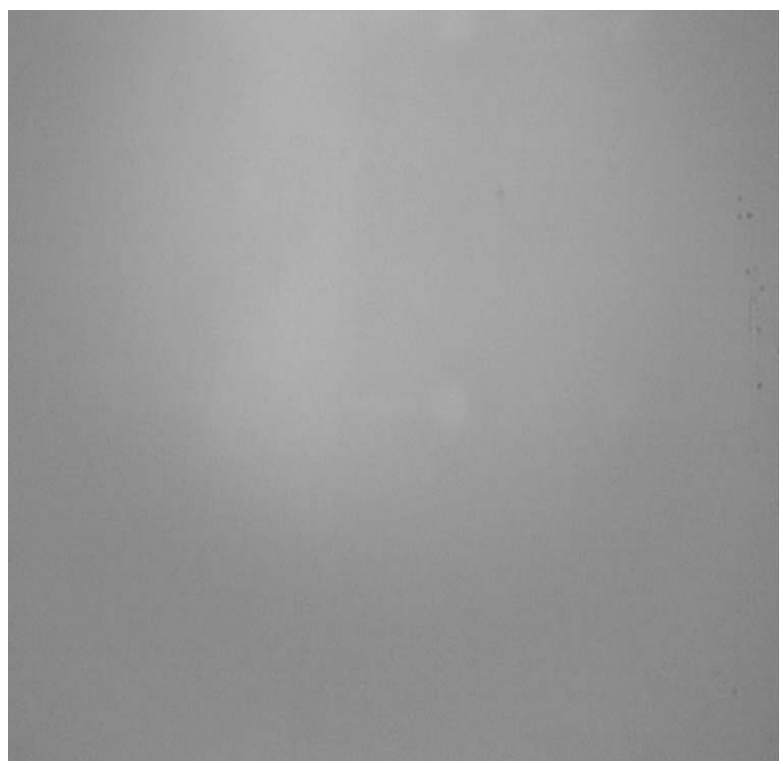

Figure 101 Image 4

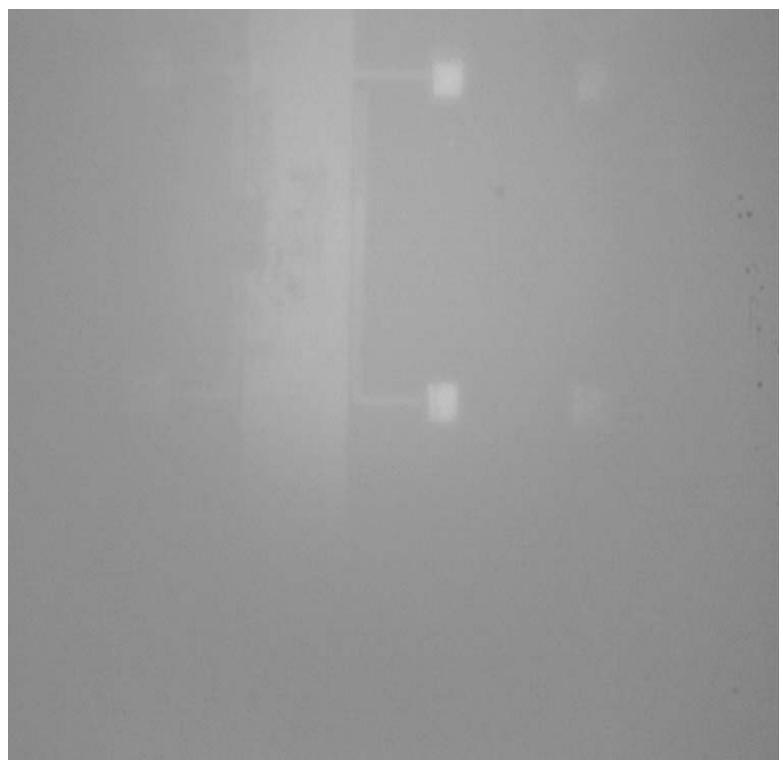

Figure 102 Image 3 


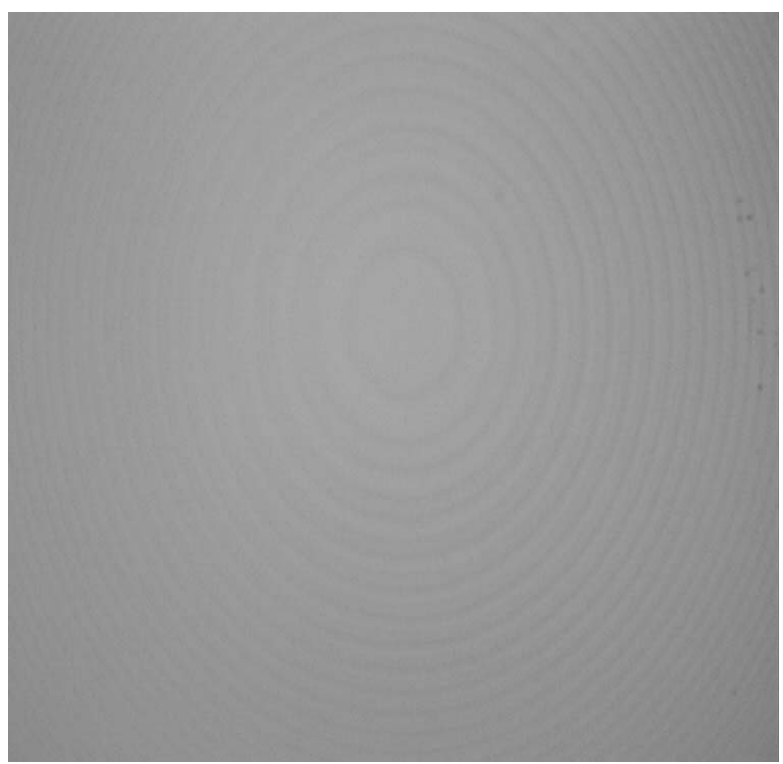

Figure 103 Bottom of DOE lens C6R1

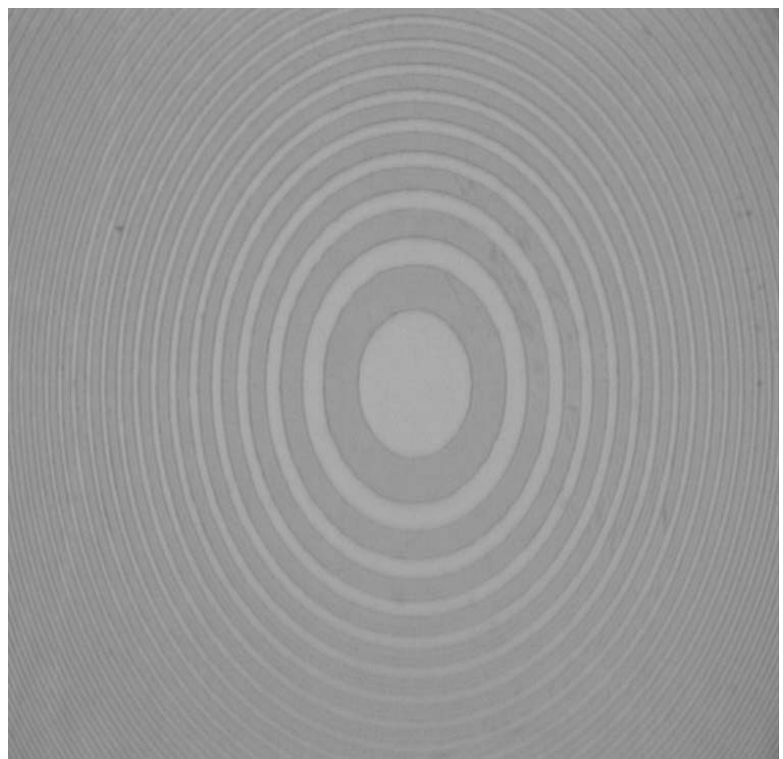

Figure 104 Top of DOE lens C6R1 


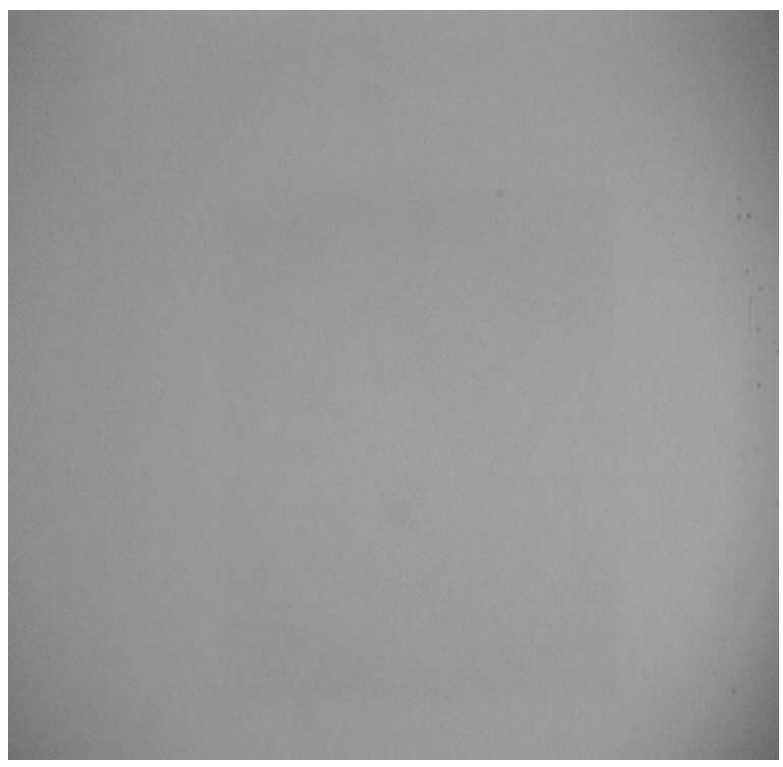

Figure 105 Image 2 reflection of lens

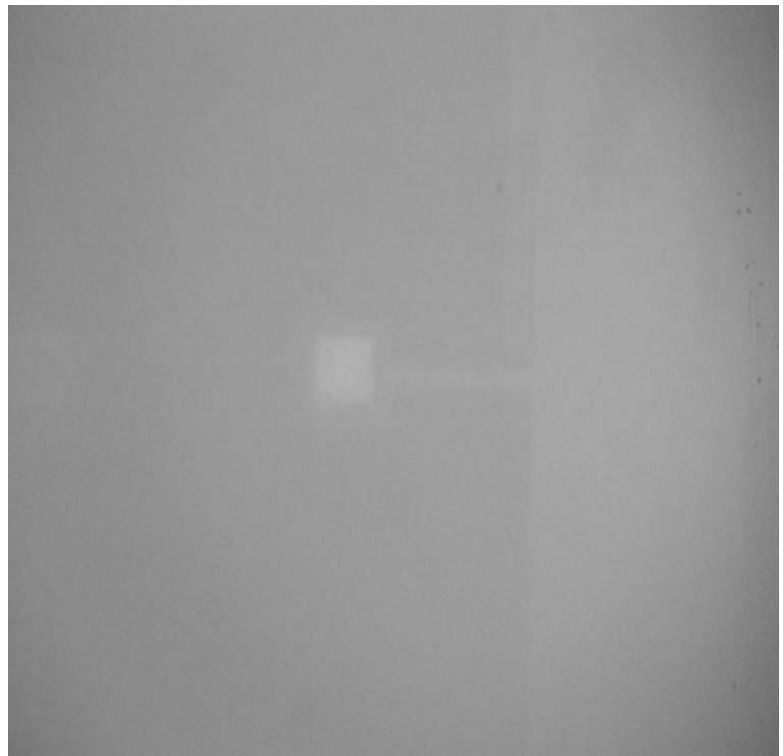

Figure 106 Image 1 primary focal point of lens C6R1 


\begin{tabular}{|l|r|r|}
\hline Object seen with 20X Objective & Distance From lens (m) & Focal Range (m) \\
\hline Image 1 & $5.4864 \mathrm{E}-03$ & $2.0320 \mathrm{E}-03$ \\
\hline Image 2 & $3.8608 \mathrm{E}-03$ & - \\
\hline Top of Lens & $0.0000 \mathrm{E}+00$ & - \\
\hline Image 3 & $-2.0320 \mathrm{E}-03$ & $1.2192 \mathrm{E}-04$ \\
\hline Image 4 & $-2.6416 \mathrm{E}-03$ & $8.1280 \mathrm{E}-05$ \\
\hline Sample & $-5.0800 \mathrm{E}-03$ & $2.5400 \mathrm{E}-03$ \\
\hline
\end{tabular}

Table 37 Tabulated distances and focal ranges of images observed with C6R1

Error! Not a valid link. outlines the distances of each image with respect to the DOE wafer. The focal range was arbitrarily picked as the outer points where each image could be discerned visually on the vibrometer computer screen.

\subsection{DOE Spot Verification and Location}

The next task was to measure the spot of the vibrometer and the spot from the DOE lens for comparison. This was approximated with the use of a micron scaled ruler etched on a glass substrate found in one of the lab microscopes. The width of the marks and distance between marks was measured using the micrometer holding the ruler and the projected image of the ruler on the vibrometer microscope. The focal point of the $20 \mathrm{x}$ objective was measured as well as the vibrometer microscope knob markings (since these were not known or found listed). There was also a channel etched in chrome that was used to test whether or not the DOE could produce a reflected signal for the vibrometer. The width of this channel was also measured.

\begin{tabular}{|c|c|}
\hline Object measured & Distance (m) \\
\hline Small knob of vibrometer (1 mark) & $1.01600 \mathrm{E}-06$ \\
\hline Large knob of vibrometer (1 mark) & $1.90500 \mathrm{E}-04$ \\
\hline Focal distance of $20 \mathrm{x}$ Objective & $1.18110 \mathrm{E}-02$ \\
\hline Width between ruler marks & $9.90600 \mathrm{E}-05$ \\
\hline Width of ruler mark & $1.27000 \mathrm{E}-05$ \\
\hline Width of Chrome Channel & $1.98120 \mathrm{E}-04$ \\
\hline Vibrometer Spot Size & $1.27000 \mathrm{E}-05$ \\
\hline to & $6.35000 \mathrm{E}-06$ \\
\hline DOE Spot Size & $4.95300 \mathrm{E}-05$ \\
\hline to & $6.35000 \mathrm{E}-06$ \\
\hline
\end{tabular}

Table 38 Spot size, Vibrometer, and Ruler measurements 
The spot observed through the vibrometer was confirmed to be produced from the DOE by observing the diffraction pattern made by the lens and the reduction and absence of the spot when moving the lens out of the laser path. The DOE spot size (diameter) has a range of 43 um because a spot size in this range gave enough reflected light for the vibrometer to take measurements. This is also the case with the vibrometer spot.

The next item to investigate was the location of the laser spot with respect to the sample and the incident laser light on the DOE. This tests the accuracy of spot placement for the on axis lenses. A random device was chosen with easily discernable features and lens C6R1 was used to deliver the laser spot to the sample. The laser spot from the vibrometer was then located on the sample near one of the sample features. The DOE lens was then placed between and centered on the vibrometer laser beam between the microscope and sample. Images 3 and 1 were observed through the vibrometer microscope to determine if the placement of the laser spot using the DOE lens was the same as that of the vibrometer alone.

The alignment of the DOE and sample with respect to the incident laser beam are important since this determines accurate delivery of the laser beam to the desired measurement location. The DOE lens was determined to be normal to the incident laser light by focusing the vibrometer microscope on the lens pattern. The DOE lens position was then altered until the entire lens pattern came into focus at the same time. The same was done with the sample to determine if the incident beam was normal to the sample. The DOE lens was then centered in the microscope field of view as well as the sample. The vibrometer laser was placed at the center of the DOE lens. The two useful images (Image 3, Image 1) observed by the DOE were then viewed to test the location of the laser spot transferred to the sample by the DOE. Figure 99 is used here to refer to the images seen. Image 3 is located between the sample and lens wafer $2 \mathrm{~mm}$ below the lens wafer. Image 1 is located $5.5 \mathrm{~mm}$ above the lens wafer. 


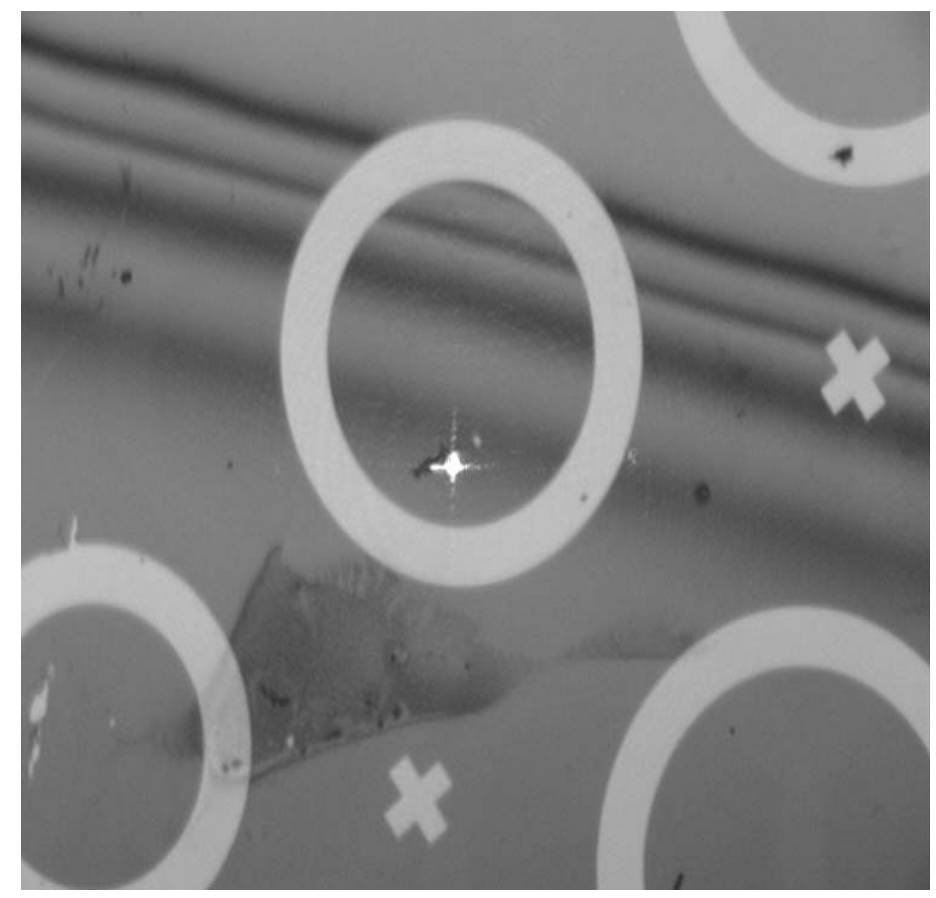

Figure 107 Random device used to determine spot placement as seen with the vibrometer.

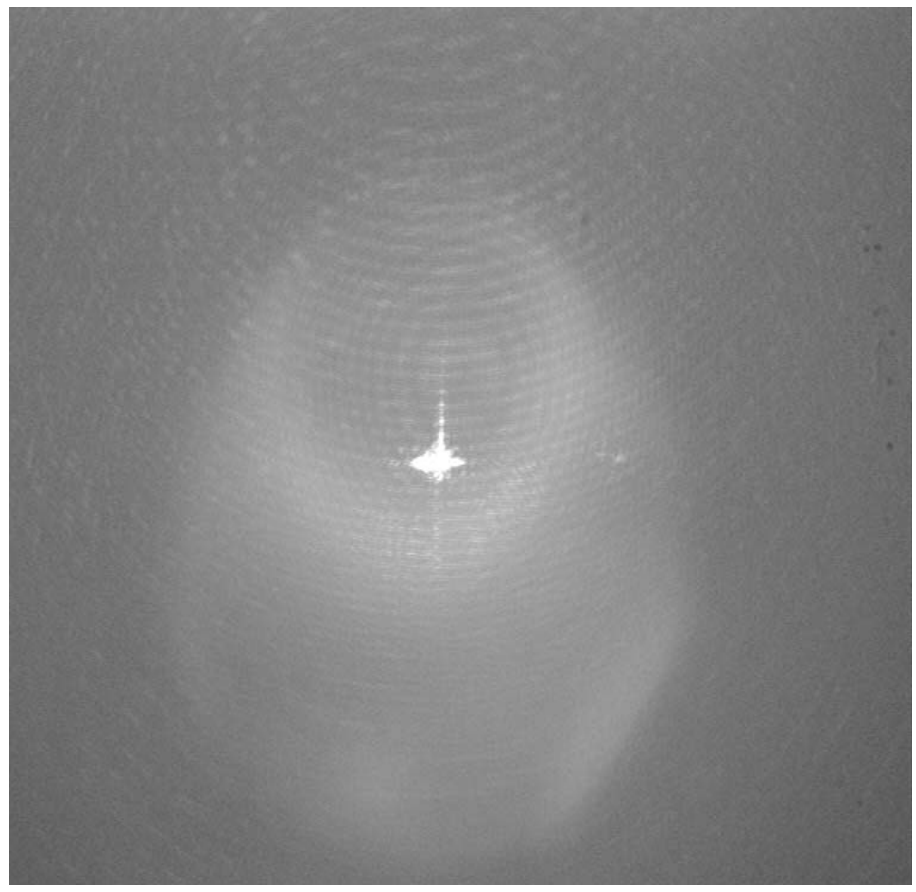

Figure 108 Image 3 showing same placement of the laser spot on the sample using the DOE. 


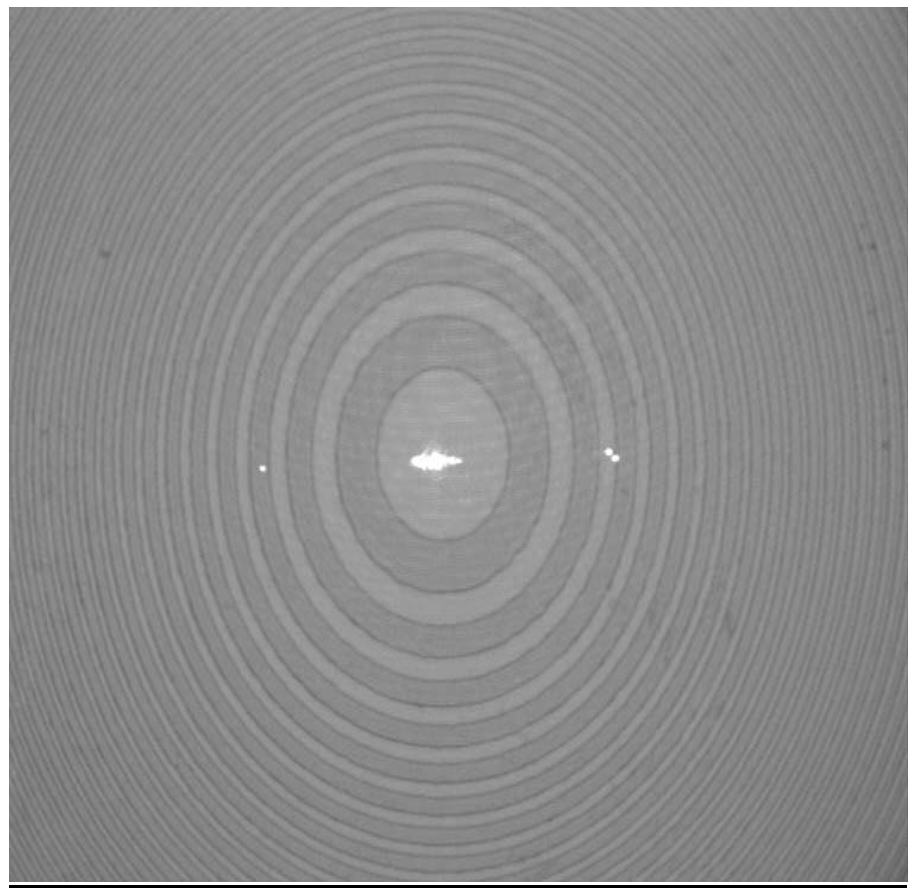

Figure 109 Location of the laser spot from the vibrometer on the DOE.

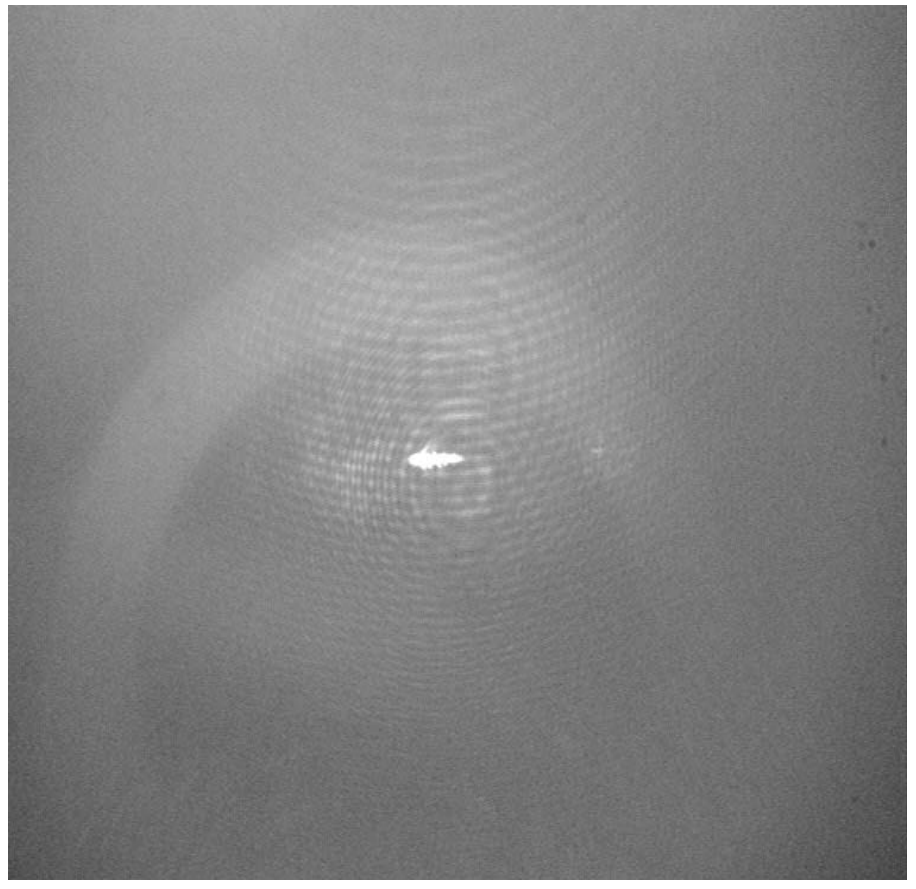

Figure 110 Image 1(inverted) showing same location of laser spot on the sample using the DOE. 
Figure 107 shows the sample as seen using only the vibrometer. Figure 108 shows Image 3 between the lens and the sample with a spot produced by the DOE that is in the same location as the spot in Figure 107. Figure 109 shows the incident beam location on the DOE used to create the spots in images 1 and 3. Figure 110 shows Image 1, primary focal point of the lens which produces an inverted image of the sample, located above the lens wafer. This image also has the same spot location from the DOE as in Figure 107. It should be noted that the images and spots observed through the DOE do not focus in the same focal plane. Image 1 comes into focus at $5.486 \mathrm{~mm}$ above the lens while the spot focuses at $6.177 \mathrm{~mm}$ above the lens. Image 3 focuses at $2.032 \mathrm{~mm}$ below the lens while the spot focuses at $2.1336 \mathrm{~mm}$ below the lens. These distances were close enough to determine the location of the observed spot relative to the sample as seen in Figure 107.

\subsection{Motion Measurements with DOE}

The device pictured below is an aluminum nitride piezoelectric device that has vertical displacement when excited with an alternating current. This device is currently being studied in our group. It is a stack structure composed of: Al - 500nm, p-type (100) Si - 300um, AlN $500 \mathrm{~nm}, \mathrm{Pt}-250 \mathrm{~nm}$. The Pt is the contact material on top of the device. The device was excited with a 40 volt, 3500 hertz signal and gave the following displacement when using the vibrometer in the single fiber mode. The output measured and shown below was averaged over 100 samples with a displacement of approximately $75-85 \mathrm{pm}$ centered at 3500 hertz. Lens C6R1 was used for the measurements.

Figure 111 shows the piezoelectric device as seen through the vibrometer without using the DOE. The dark line to the device is the probe that delivered the excitation voltage. Figures 112 and 113 show the vertical displacement measured when using only the vibrometer.

Figure 114 shows the measured background noise ( 2.5 pico-meters) produced when there is no excitation of the device. The noise is likely due to mechanical vibrations from the work bench. Mechanical vibrations can be observed on the output graph of the vibrometer by moving the device manually. 


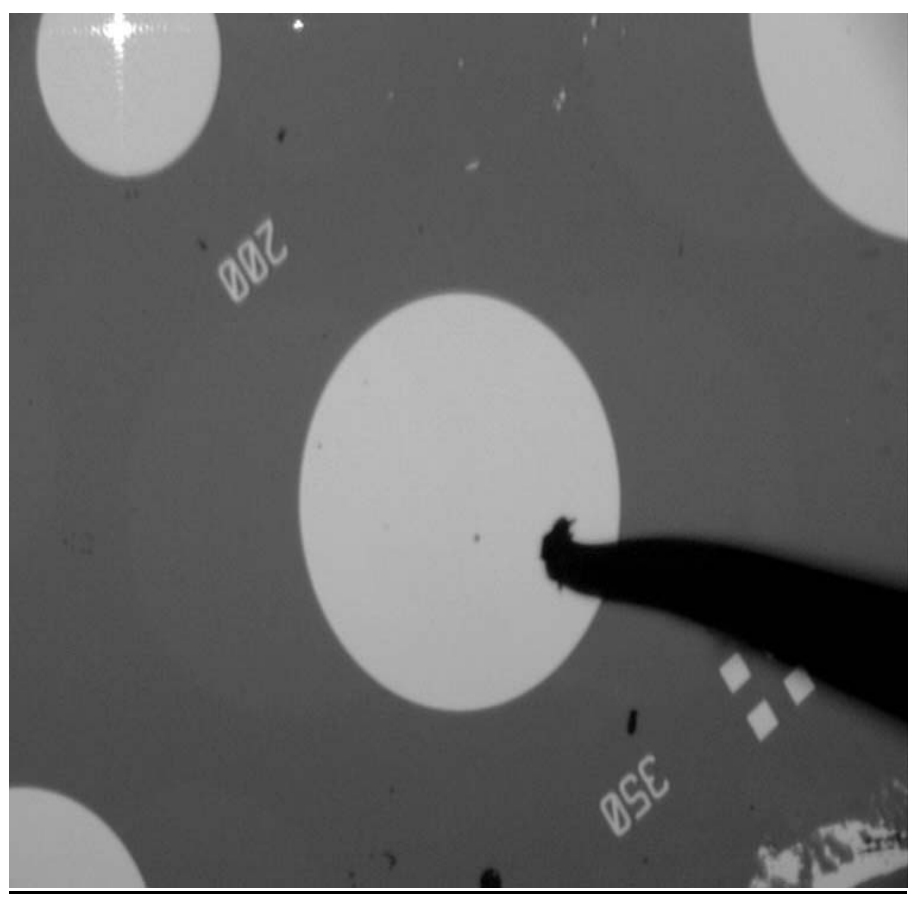

Figure 111 Test sample as seen through the vibrometer only.

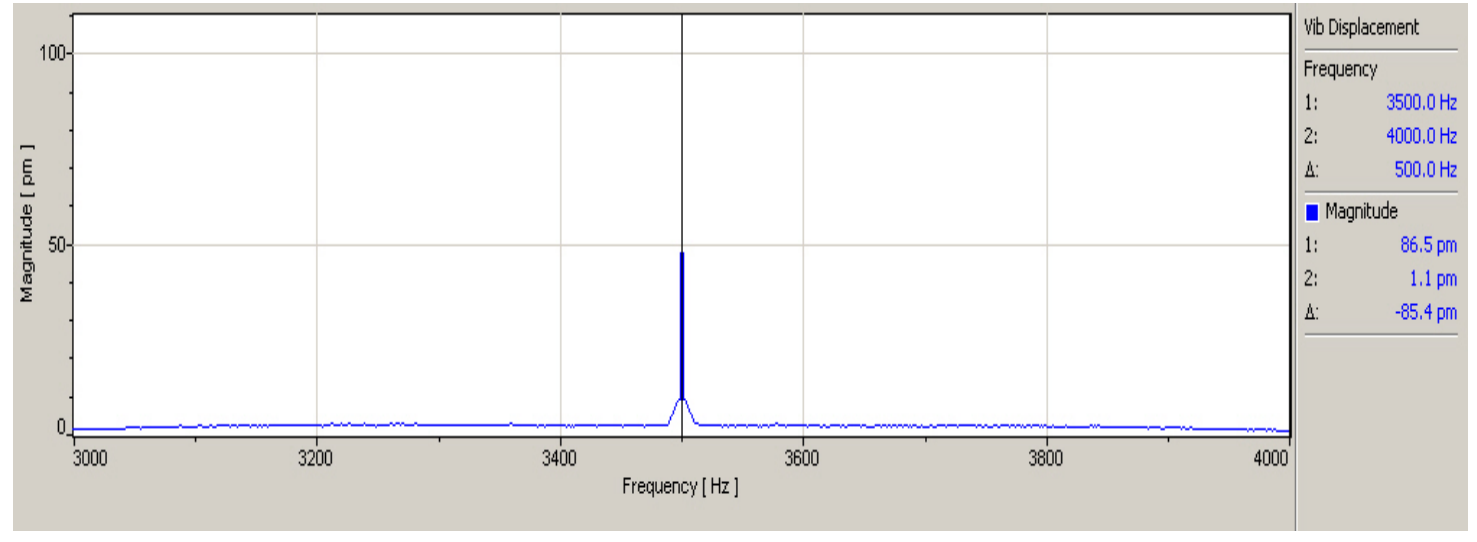

Figure 112 Displacement measured with vibrometer only over 3-4 kilohertz. 


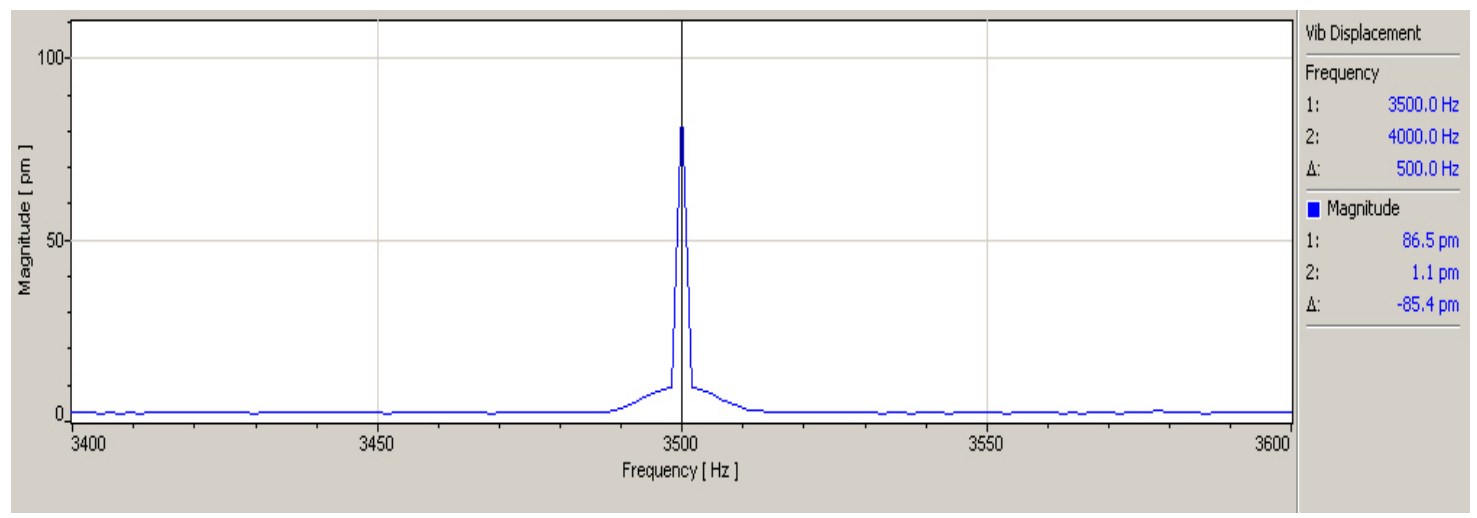

Figure 113 Displacement measured with vibrometer only over 3.4-3.6 kilohertz.

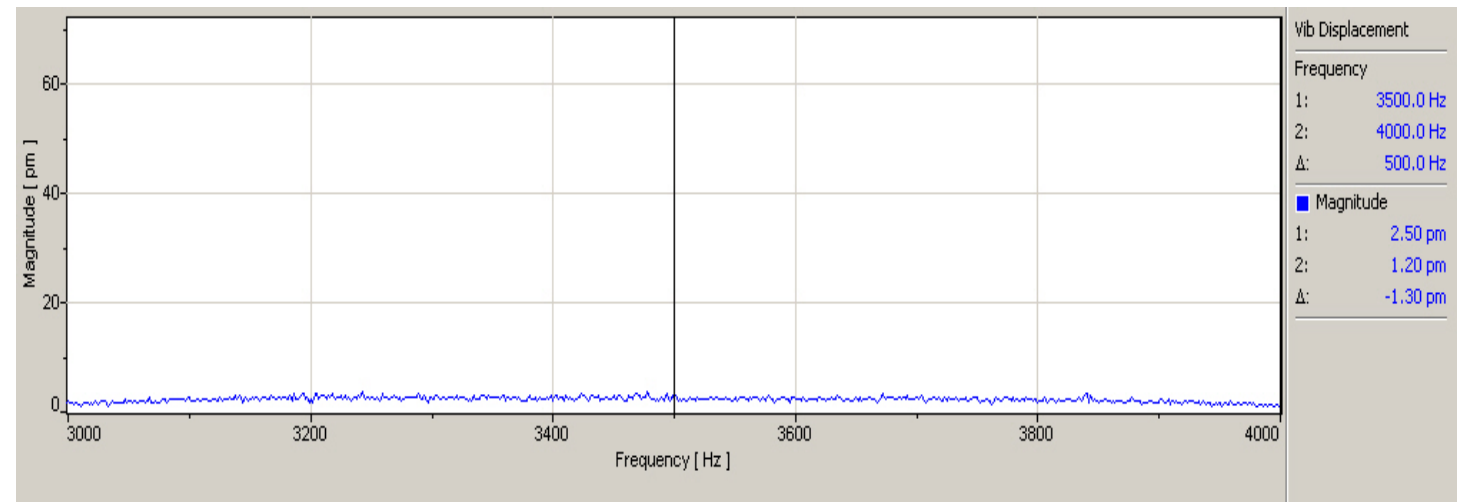

Figure 114 Background noise (2.5pm) measured with vibrometer only.

Figures 115 and 116 show the displacement measured at the Image 1 location (between the DOE lens and sample) when using the DOE.

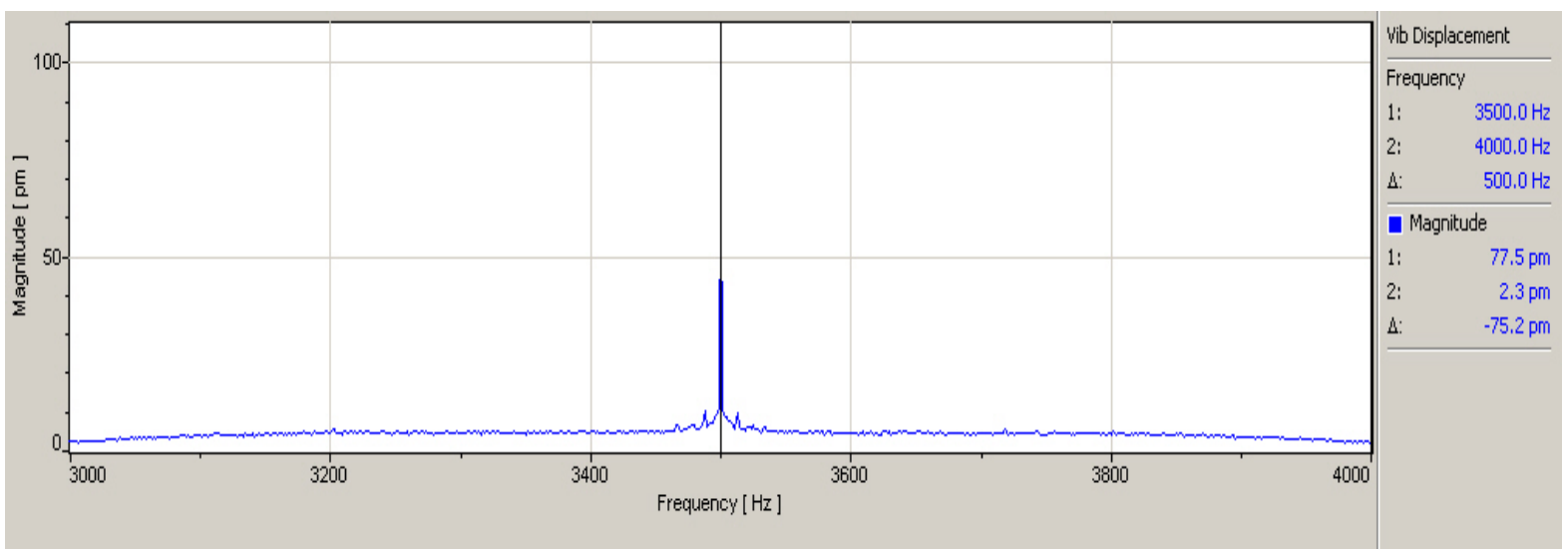

Figure 115 Displacement measured at Image 3 location using DOE from 3-4 kilohertz. 


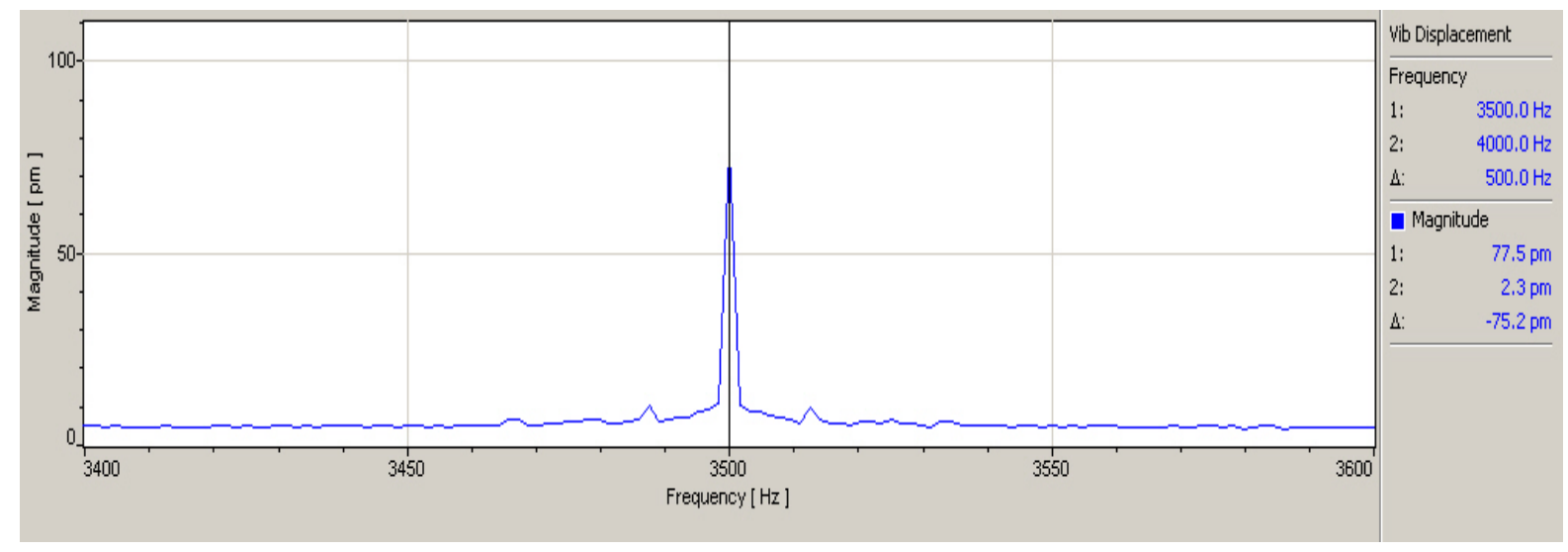

Figure 116 Displacement measured at Image 3 location using DOE from 3.4-3.6 kilohertz.

Figures 117 and 118 show the displacement observed at the Image 1 location when using the DOE(the primary focal point above the DOE lens). Figure 119 shows the measured noise floor, 3.9 picometers, at this location using the DOE when there is no excitation of the device.

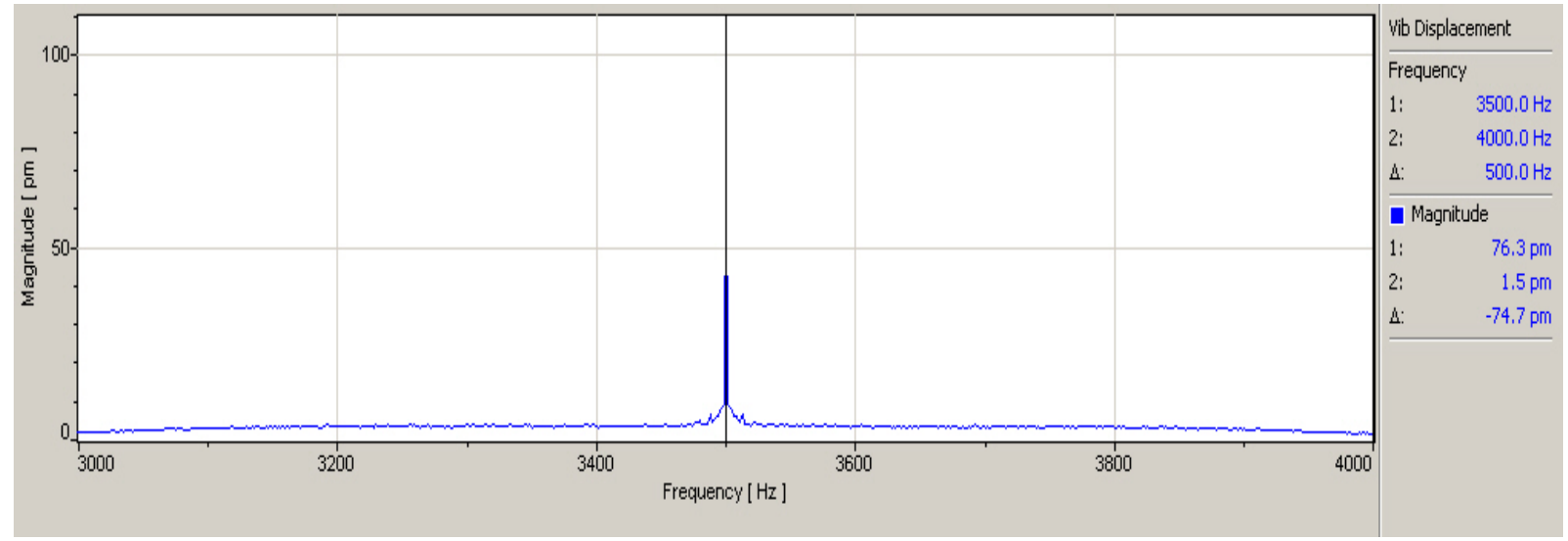

Figure 117 Displacement measured at Image 1 location using DOE from 3-4 kilohertz. 


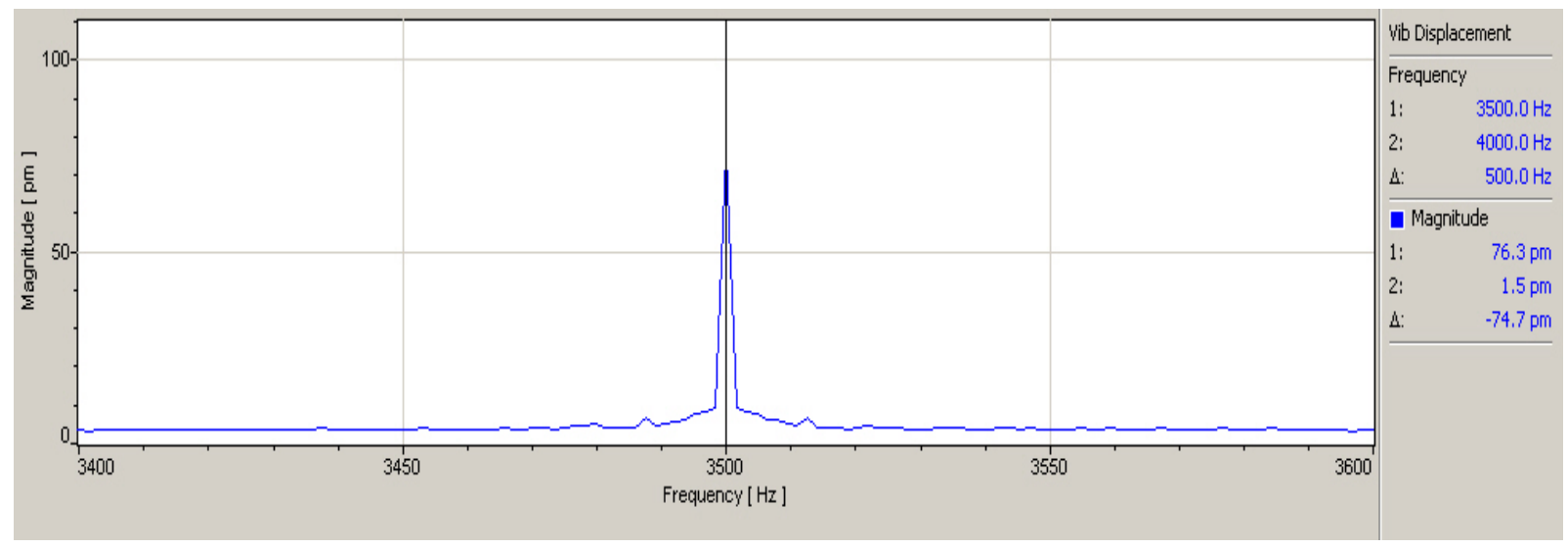

Figure 118 Displacement measured at Image 1 location using DOE from 3.4-3.6 kilohertz.

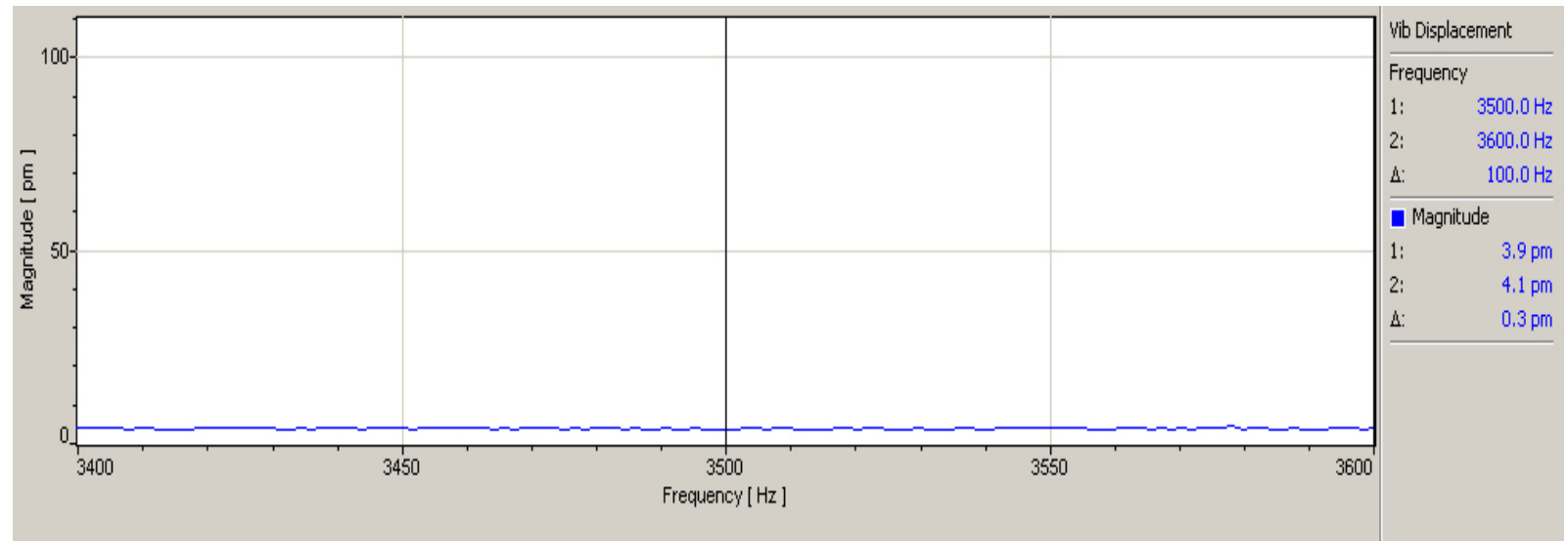

Figure 119 Background noise (3.9pm) observed at Image 1 location with no excitation of device.

The displacement of the device as seen through the DOE was comparable to that observed when using the vibrometer alone. A comparison of the displacement of each of these three locations is listed below:

\begin{tabular}{l|r|r|r|r|}
\multirow{4}{*}{$\begin{array}{l}\text { Peak Displacement } \\
\text { Noise }\end{array}$} & Vibrometer $(\mathrm{pm})$ & DOE Image 3 & $(\mathrm{pm})$ & DOE Image 1 (pm) \\
\cline { 2 - 5 } & $\mathbf{8 6 . 5}$ & $\mathbf{7 7 . 5}$ & $\mathbf{7 6 . 3}$ \\
\cline { 2 - 5 } SNR & $\mathbf{2 . 5}$ & $\mathbf{3 . 9}$ & $\mathbf{3 . 9}$ \\
\cline { 2 - 5 } & & & & 19.9 \\
\hline
\end{tabular}

Table 39 Vibrometer displacement of piezoelectric device. 
It should be noted that the SNR of the measurements taken with the DOE are close to that of lens C6R1 which was found to be 20.5 . This could account for the measured $10 \%$ decrease in peak displacement and 56\% noise floor increase. The overall width and shape of the observed displacement peak was unchanged. The observed differences of the measured DOE displacement compared to the vibrometer-only displacement could arise from the fact that both the vibrometer and the DOE lenses work because of phase shifted light. The above measurements are a strong indication that the fabricated DOE lenses can be used for motion detection of MEMS devices. 


\section{Conclusions}

The increasing use of MEMS devices creates a need to monitor microstucture motion in order to determine their operational status over the system's lifetime, especially in the case of safety critical; systems. Integrated optics will play a major role in achieving the hybrid-integration of components necessary for establishment of the optical systems necessary for in-situ lifetime optical monitoring. A key optical component to be fabricated and tested is the optical probe beam focusing system. Previous work by our group had led to the design and fabrication of binary diffractive phase lenses to be evaluated as integrated optical elements for this purpose. The current work experimentally and theoretically determines if the fabricated lenses met the intended design criteria and could be viable for integrated optical monitoring of MEMS devices.

The characterization of the DOE lenses in Chapter 8 has shown that the lenses meet their design criteria and have the necessary specifications to be used as integrated optical elements. The use of lens C6R1 shows these lenses can produce the desired 5 um spot size though the desired focal lengths are not within specifications. The on-axis lenses have proven to be close to $30 \%$ efficient. It is believed this could be made to approach the optimal value of $40 \%$ by 1.) improving the diffractive lens structure through conducting fabrication in the new clean room environment and 2.) by refining the etch of the glass substrate to remove surevace roughness of the etched layer. The location of the laser spot produced by the on-axis DOE lenses was shown to be accurately placed in its intended location. The SNR of 20 for lens C6R1 has proven sufficient to deliver, return, and analyze a laser signal to a MEMS device. The returned signal was shown in Chapter 10 to be strong enough for use by the vibrometer system to detect motion of a piezoelectric device. The characterization and vibrometer experiments of the DOE lenses in Chapters 8 and 10 leads to the conclusion that these lenses are reasonable candidates for inclusion in prototype integrated optical monitoring systems. 


\section{Future Work}

This research concludes has compiled the experimental specifications for the on-axis DOE lenses and the verified that they can be used in conjunction with the vibrometer to track motion of MEMS devices. The next step for integrated motion detection would be to determine how to couple an optical fiber to a lens and deal with alignment issues associated with testing a device on chip without assistance of the vibrometer microscope for alignment. Also, fabrication of the lenses on chip with the MEMS devices and their alignment must be addressed. Re-making the lenses in the clean room may also improve efficiency. Another area to investigate is the off-axis lenses. These will be very useful in an integrated optical monitoring design. All of the previous experiments used on the on-axis lenses can be applied to the off-axis lenses. In addition to those measurement, the off axis spot locations should be measured for the off-axis lenses. 


\section{Bibliography}

[1] W.B. McCormick, "Diffractive Optical Lens Design and Fabrication for Integrated Monitoring of Microelectromechanical Lateral Comb Resonators", Masters Thesis, West Virginia University (2004).

[2] J.M. Dawson. "Integrated Through-Wafer Monitoring of MEMS for Closed-Loop Control", PhD. Dissertation, West Virginia University (2002).

[3] J.M. Dawson. "Through-Wafer Interrogation of MEMS Device Motion”, Masters Thesis, Wext Virginia University. (1999)

[4] B.E.A. Saleh and M.C. Teich, Fundamentals of Photonics, ISBN 0471839655, WileyInterscience, New York, 1991.

[5] S. Ramo, J.R. Whinnery, T. Van Duzer, Fields and Waves in Communication Electromagnetics, ISBN 0471585513, John Wiley \& Sons Inc. New York 1994.

[6] M. Born, E. Wolf, Principles of Optics, $7^{\text {th }}$ edition, ISBN 0521642221, University Press, Cambridge, 1999.

[7] S.A. Campbell, The Science and Engineering of Microelectronic Fabrication, 2nd edition, ISBN 0195136055, Oxford University Press, New York, 2001.

[8] S.O. Kasap, Optoelectronics and Photonics:Principles and Practices, ISBN 0201610876, Prentice Hall, New Jersey, 2001.

[9] M.C. Hutley, Diffraction Gratings, ISBN 0123629802, Academic Press Inc., New York, 1982.

[10] J. Ojeda-Castaneda and C. Gomez-Reino, eds. Selected Papers on Zone Plates. Vol. MS 128. Washington: SPIE Optical Engineering Press, 1996.

[11] S. H. Lee, ed. Computer-Generated Holograms and Diffractive Optics. Vol. MS 33. Washington: SPIE Optical Engineering Press, 1996.

[12] Spiricon Neutral Density Data. On-line.10 Apr. 2006. Available http://www.spiricon.com/techinfo/product_data/beam_sampling/lbs100.shtml

[13] Allen Cary, "Beam Profiling", On-line. 10 Apr. 2006. Available 
http://www.photon-inc.com/BeamProfiling.pdf

[14] L. D. Konduparthi, "Optical Detection of Multiple Faults of a MEMS Based Linear Comb Resonator", Masters Thesis, West Virginia University, (2006).

[15] Schott North America website, Borosilicate33 chemical composition, On-line. 10 Apr. 2006. Available

http://www.us.schott.com/whitegoods/english/products/borofloat/attribute/chemical/in dex.html.

[16] Schott North America website, Borosilicate33 UV transmission properties, On-line. 10 Apr. 2006. Available http://www.us.schott.com/whitegoods/english/products/borofloat/attribute/optical/ima ges/transmit_uv range.pdf.

[17] Schott North America website, Borosilicate33 IR transmission properties, On-line. 10 Apr. 2006. Available http://www.us.schott.com/whitegoods/english/products/borofloat/attribute/optical/ima ges/transmit_ir_range.pdf.

[18] Schott North America website, Borosilicate33 dispersion properties, On-line. 10 Apr. 2006. Available http://www.us.schott.com/whitegoods/english/products/borofloat/attribute/optical/ima ges/dispersion borofloat.pdf.

[19] Spiricon website, Online, 10 Apr. 2006. Available http://www.spiricon.com/techinfo/product_data/beam_sampling/LBS100.pdf

[20] Polytec. Laser Doppler Vibrometer Users Manual (Controller OFV-3001, Sensor Heads OFV-303/353 OFV-511/512.

[21] M. Bottema. "Fresnel Zone-Plate Diffraction Patterns" Selected Papers on Zone Plates. Ed. J. Ojeda-Castaneda and C. Gomez-Reino. Vol. MS 128. Washington: SPIE Optical Engineering Press, 1996. 99.

[22] G.J. Swanson and W.B. Veldkamp. "Infrared applications of diffractive optical elements" Computer-Generated Holograms and Diffractive Optics. Ed. S. H. Lee. Vol. MS 33. Washington: SPIE Optical Engineering Press, 1996. 359. 